\begin{abstract}
Title of dissertation:

SCHOOL READINESS OF MALTREATED PRESCHOOLERS AND LATER SCHOOL ACHIEVEMENT: THE ROLE OF EMOTION REGULATION, LANGUAGE, AND CONTEXT

Carlomagno C. Panlilio, Doctor of Philosophy, 2015

Directed By:

Associate Professor Brenda Jones Harden, Department of Human Development and Quantitative Methodology
\end{abstract}

Academic achievement is an important indicator for the well-being of children with a history of maltreatment. Unfortunately, many of these children fall behind their non-maltreated peers in measures of academic performance, and the achievement gap between these groups is increasing. Attempts to close this gap at later ages can prove to be challenging. The focus on early childhood as a developmental period to direct research and intervention efforts holds promise. Early childhood is a critical time for the ongoing development of emotion regulation, which is an important domain of school readiness. For young maltreated children, however, specific individual-level and context-level factors need to be considered in understanding how emotion regulation development proceeds. That is, the placement experiences for these young children vary greatly in the cognitive stimulation, emotional support, and stability they provide. Qualitative differences in these context-level factors can place children in different trajectories of development. These varying trajectories, in turn, may place young maltreated children in different pathways that lead to different academic outcomes in later grades.

The goals of this study then were to: 1) examine the growth curves and determine the functional form of emotion regulation across time, beginning with early childhood when first contact with Child Protective Services (CPS) occurred; 2) identify latent classes based on 
developmental patterns of emotion regulation for maltreated preschool-aged children; 3) examine developmental differences based on individual-level and context-level factors specific to the experiences of young maltreated children; and 4) elucidate the different pathways to later academic achievement. This study utilized data from the National Survey of Child and Adolescent Well-Being (NSCAW I) study, which was a nationally-representative study that employed a complex probability sampling framework that provided estimation of national-level parameters. Data analyses used latent growth curve models, latent class analyses, and latent transition analyses to answer the goals stated above. Results indicated stability and change in emotionally regulated vs. emotionally dysregulated latent classes across 4,5 , and $6 \frac{1}{2} 2$ years of age. Moreover, children classified as emotionally dysregulated at age $61 / 2$ scored significantly lower than children who were classified as emotionally regulated on measures of reading and math achievement by age 10. Policy implications for child welfare and early childhood education are presented. 


\title{
SCHOOL READINESS OF MALTREATED PRESCHOOLERS AND LATER SCHOOL ACHIEVEMENT: THE ROLE OF EMOTION REGULATION, LANGUAGE, AND CONTEXT
}

\author{
By \\ Carlomagno C. Panlilio \\ Dissertation submitted to the Faculty of the Graduate School of the \\ University of Maryland, College Park, in partial fulfillment \\ of the requirements for the degree of \\ Doctorate of Philosophy \\ 2015
}

Advisory Committee:

Dr. Brenda Jones Harden, Chair

Dr. Jeffrey Harring

Dr. Christy Tirrell-Corbin

Dr. Judith Torney-Purta

Dr. Elaine A. Anderson 


\section{Dedication}

To my dear wife, Dora Hunter, you have been there with me from the very beginning. It is with your love, encouragement, and patience that I reached the finish line. You are my

lighthouse. I am forever grateful for your love and support. To Theo, you may be too young to read this now, but know that you have taught me so much, especially the value of balance. 


\section{Acknowledgements}

This has been a long journey filled with ups and downs throughout the years. I would not have made it this far without the love, support, encouragement, and patience from my family, friends, mentors, and colleagues. Thank you all so much for being a huge part of my life as a graduate student.

I would like to thank my advisor, Dr. Brenda Jones Harden, for taking me under her wings. Had it not been for you, I would not have considered returning for my Ph.D. Our common pathway and your current success have been a great inspiration. I truly admire the work that you do to help children and families at risk. You provide a voice to those who have none. I am so grateful for your patience with me, especially when I am always hurrying through. Thank you so much for your mentorship, support, advice, and friendship.

To Dr. Judith Torney-Purta, I am ever so grateful for all that you have done. You have been instrumental in getting my career started as a Ph.D. student and had it not been for that phone call, my journey would have taken me somewhere else. Thank you for all of your wisdom, guidance, support, and friendship.

Dr. Jeff Harring, thank you for being willing and able to listen and read about my rants in those moments when I felt like I was going around in circles. Your methodological expertise has been an inspiration for me to seek out and learn new ideas beyond my quantitative comfort zone. Thank you!

I would also like to thank Dr. Christy Tirrell-Corbin. It has been a pleasure to have been working with you from the beginning. Had it not been for the opportunities and knowledge you provided, I would not have become familiar with, and interested in, learning more about the field of early childhood education. 
To Dr. Elaine Anderson, thank you for your feedback and guidance throughout the dissertation process. It has been great getting to work with you again after all these years. Your perspective and expertise in families have been invaluable.

I would like to thank my former mentors who have been, and still are, a big part of my life for the support and encouragement they have provided through the years: Dr. Ned Gaylin, Dr. Leigh Leslie, Dr. Norm Epstein, Dr. Carol Werlinich, and Dr. Michael Connor.

Thank you to all of my fellow grad students, friends who have all been through the same experience as we laughed and cried through those semesters: Katie Muenks, Erica Zippert, Colleen Morrison, Aimee Duncan, Melissa Duchene, Meryl Barofsky, Shelby Cooley, Maureen Wimsatt, Sara Fitt, Sarah Eason, Ting Zhang, Danette Morrison, Nikki Denmark, Emily Rosenzweig, and Alexis Williams.

I would like to thank Chapin Hall and the Doris Duke Fellowship for the Promotion of Child Well-being for supporting my research and sharing the same goal of helping the most vulnerable children and families. The experience I've gained and the friends I've met through the fellowship have been invaluable. I would especially like to thank Dr. Melissa Lim Brodowski, whose expertise in Child Welfare policy have helped broaden my perspective. I would also like to thank Dr. Deb Daro and Lee Ann Huang. You have both been very supportive and encouraging of the interdisciplinary perspective that is very important in addressing the needs of at-risk children and families.

To the children and families who trusted me to understand their experiences, struggles, and hopes. You have provided the questions, lessons, and motivation to push forth and bring your experiences in the forefront of my research. I thank you and will ensure that your voices and struggles are heard. 
Finally, I would like to thank my mother and my grandmother who have taught me the value of education, dedication, hard work, perseverance, and kindness. Thank you for all that you have done for me. 


\section{Table of Contents}

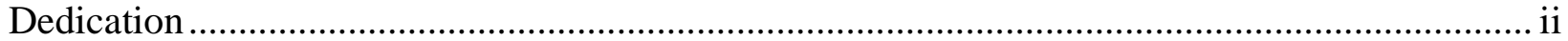

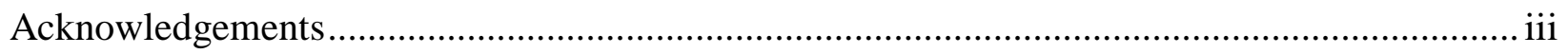

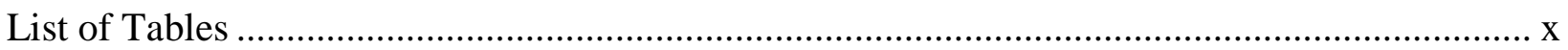

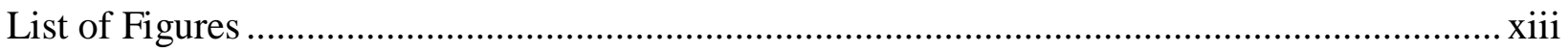

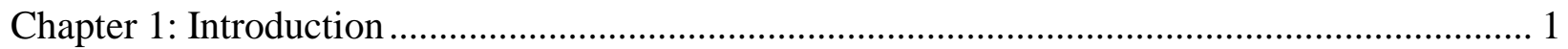

Maltreatment in the Early Childhood Period ...................................................................... 3

Theoretical Background and Conceptual Model........................................................................ 4

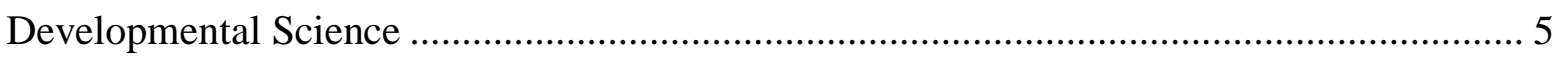

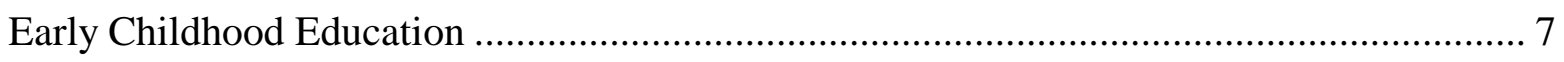

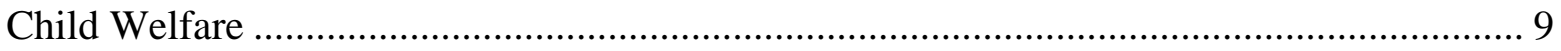

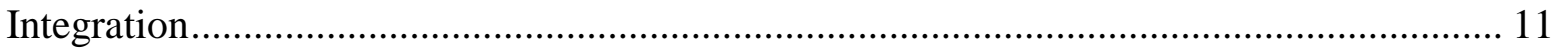

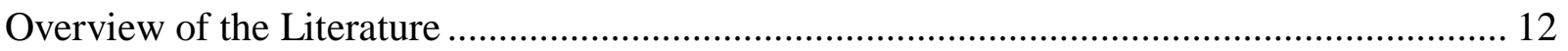

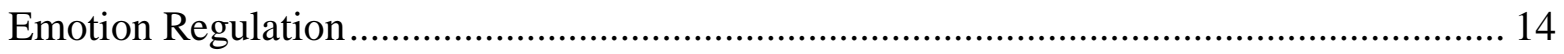

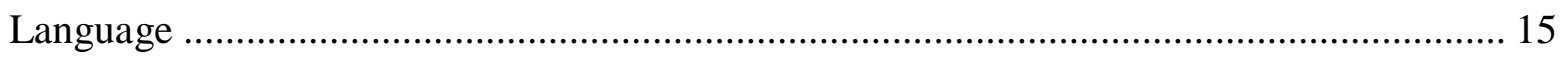

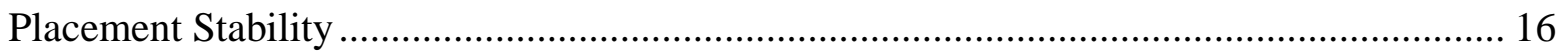

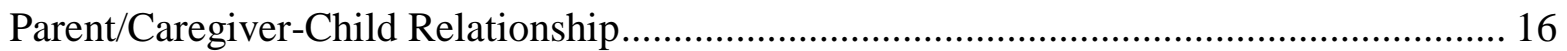

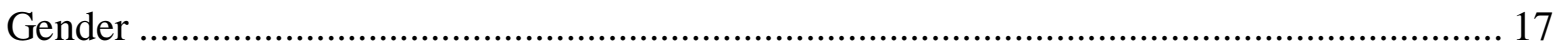

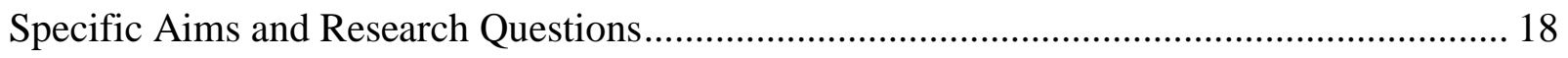

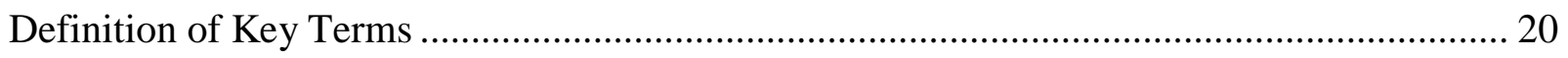

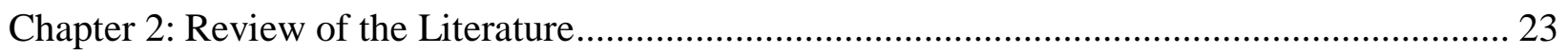

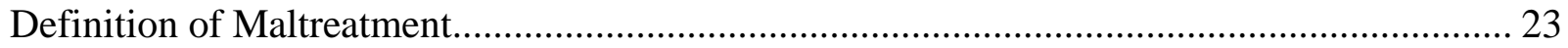

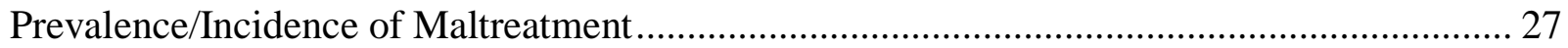

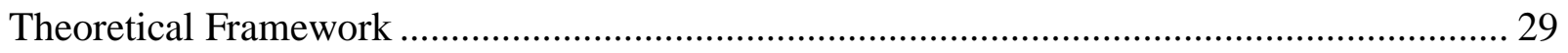

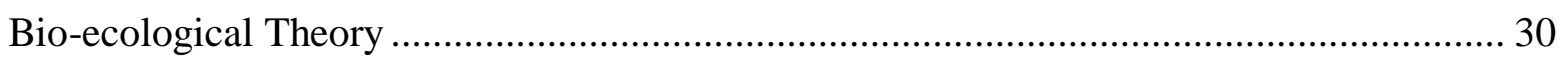

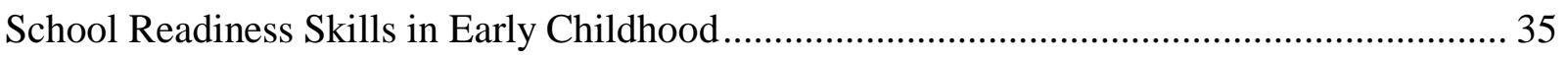

Academic Achievement: Elementary to Adolescence .......................................................... 41

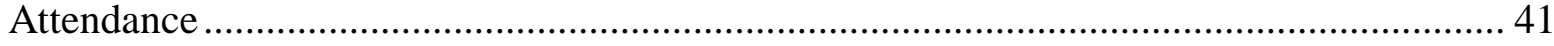

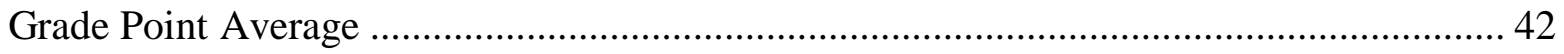

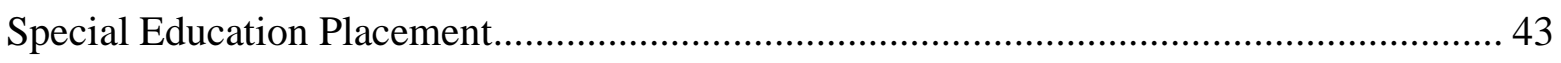

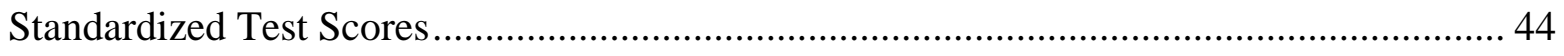

Bio-ecological Factors and Maltreated Children ………........................................................ 46

Definition of Emotion Regulation .................................................................................... 47 
Emotion Regulation and Maltreatment........................................................................... 50

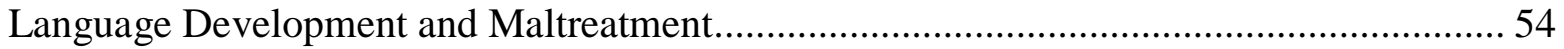

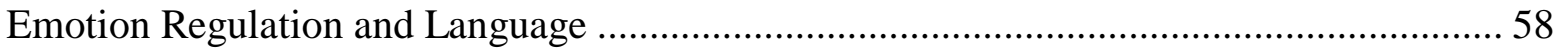

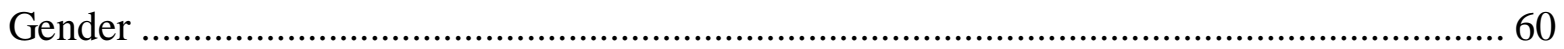

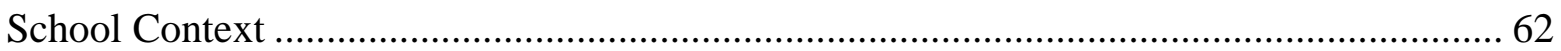

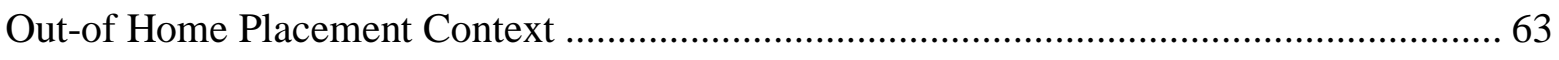

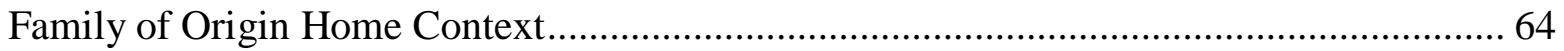

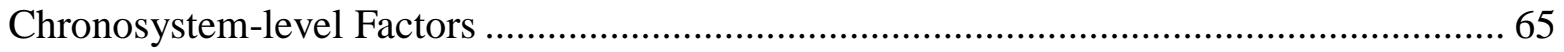

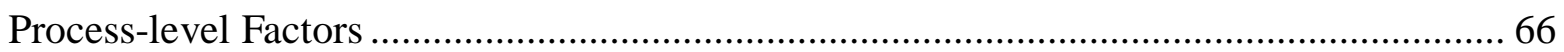

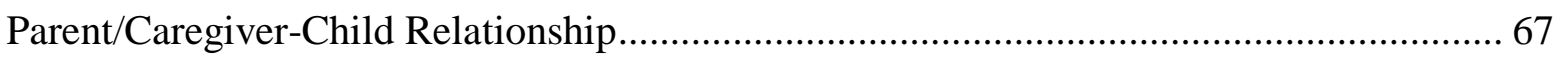

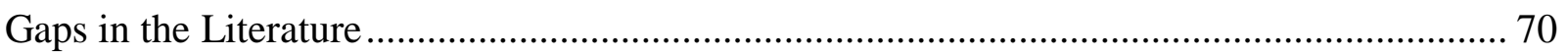

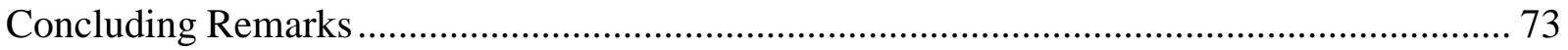

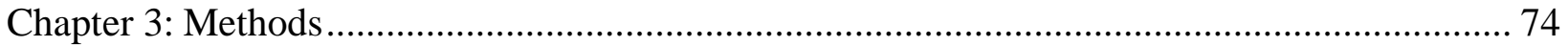

The National Survey of Child and Adolescent Well-Being ....................................................... 74

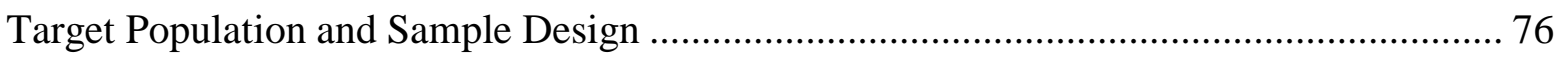

Bias Due to Sampling Frame Non-coverage Error............................................................ 79

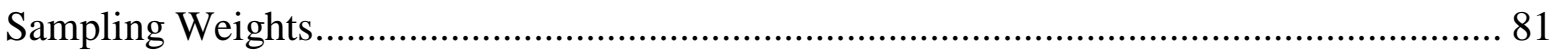

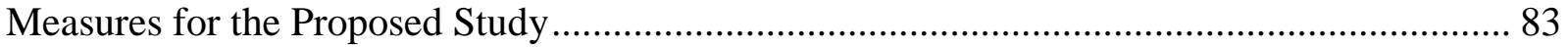

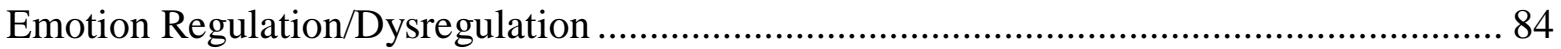

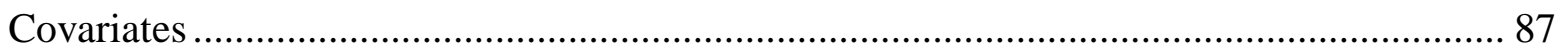

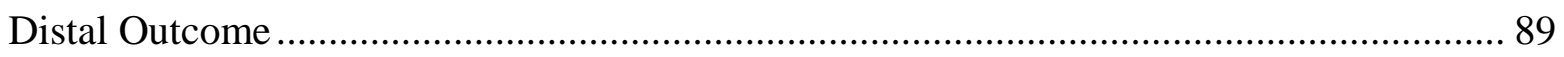

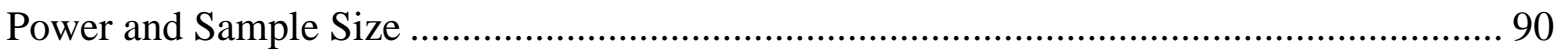

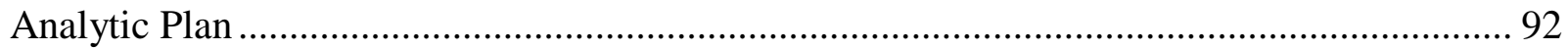

Exploratory Data Analyses ......................................................................................... 93

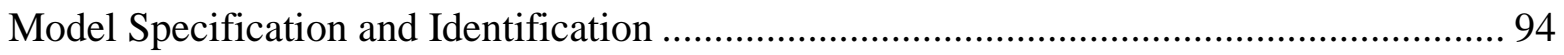

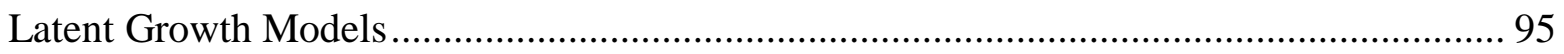

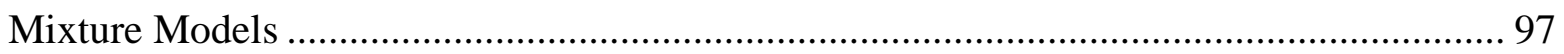

Evaluation of data-model fit .......................................................................................... 99

Analysis of Research Questions ......................................................................................... 101

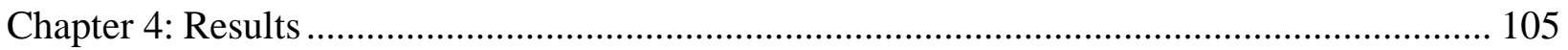

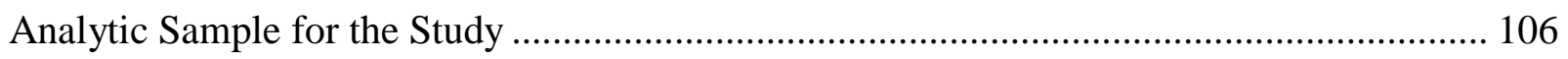

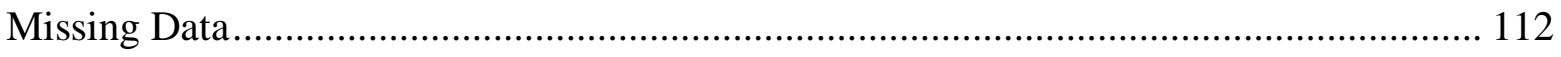




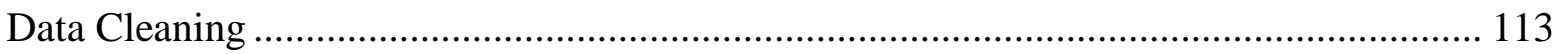

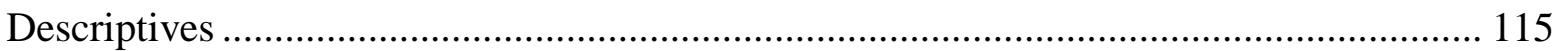

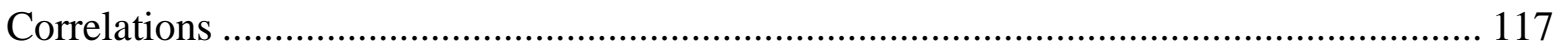

Measurement Models for Emotion Dysregulation ........................................................... 118

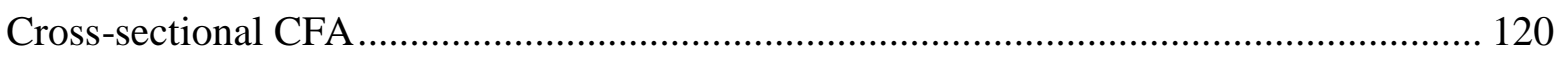

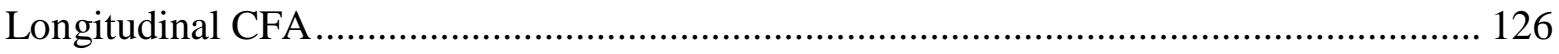

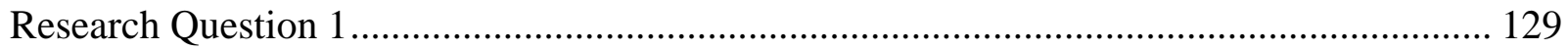

Latent growth models of emotion dysregulation............................................................. 129

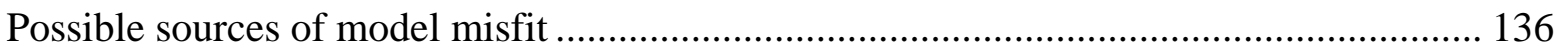

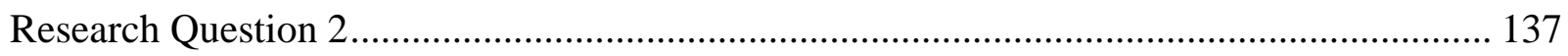

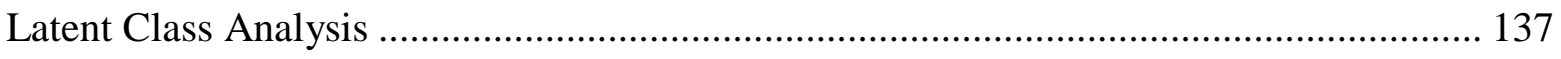

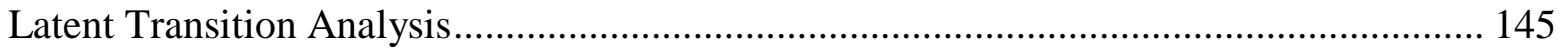

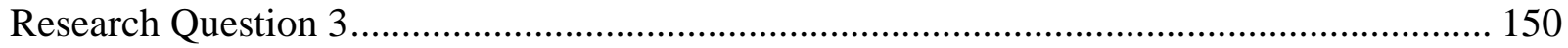

Research Question 4.......................................................................................................... 157

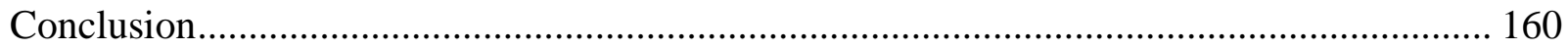

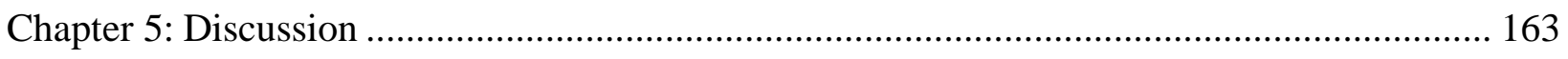

Summary and Interpretation of Results............................................................................. 163

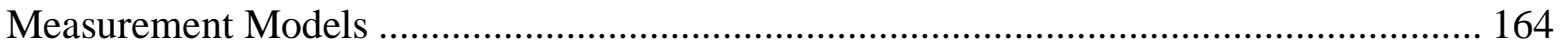

Development of Emotion Dysregulation across Time .................................................... 165

Individual-level and Context-level Factors …………................................................... 177

Developmental Pathways to Academic Achievement ...................................................... 181

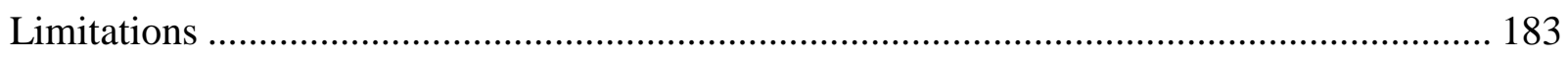

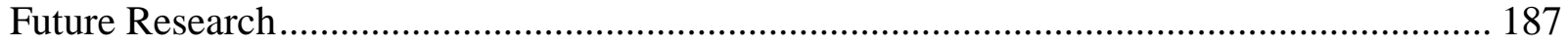

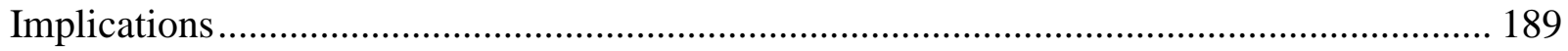

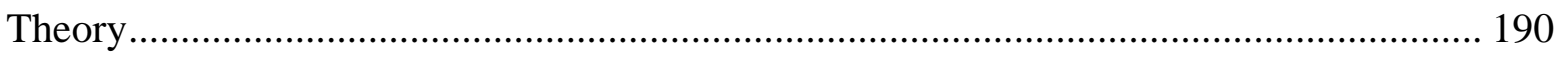

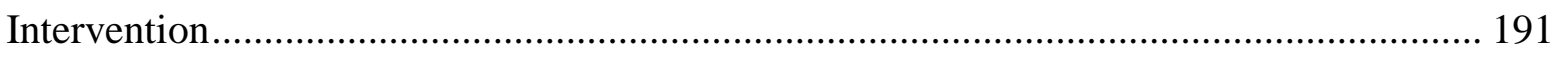

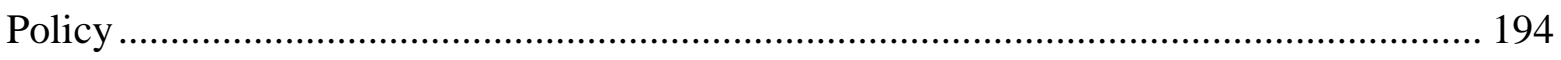

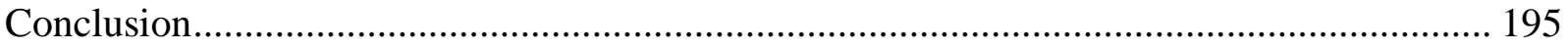

Appendix A: Sampling Domains for the NSCAW CPS Sample ................................................ 197

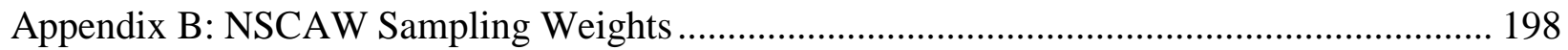

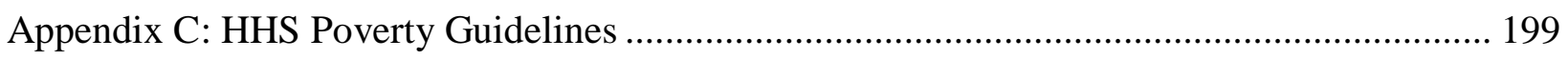

Appendix D: Cross-tabulation for Poverty Estimation .......................................................... 201 
Appendix E: Missing Data Pattern Frequencies ......................................................................... 206

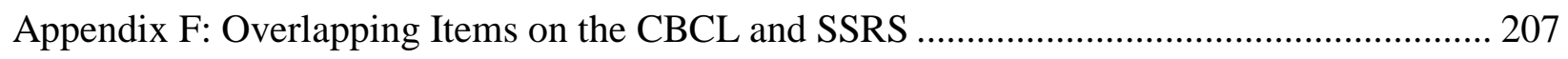

Appendix G: Final CBCL Items Used in the Study ............................................................. 210

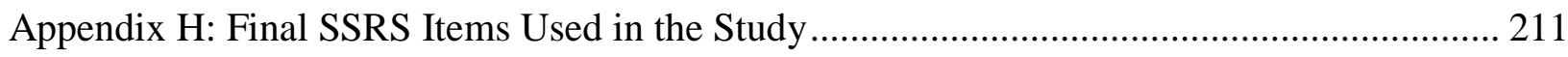

Appendix I: Boxplot Figures with Outliers ...................................................................... 212

Appendix J: Intercorrelations of Study Variables................................................................ 214

Appendix K: Item Probabilities from LCA Models ........................................................... 232

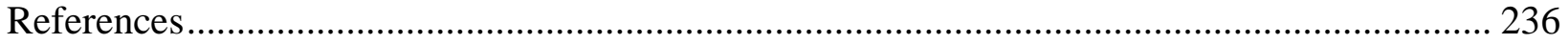




\section{List of Tables}

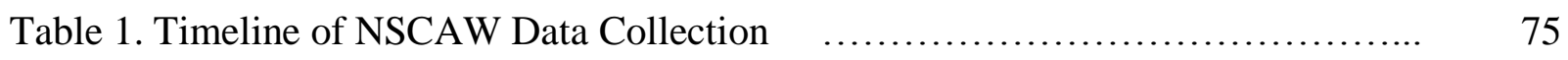

Table 2. Sample Size Requirement at Different RMSEA Levels $\quad \ldots \ldots \ldots \ldots \ldots \ldots \ldots . . . \ldots 1$

Table 3. Distribution of Age Groups in the Total NSCAW CPS $\quad \ldots \ldots \ldots \ldots \ldots$

Sample

Table 4. Child Demographic $\quad$.................................. 107

Information

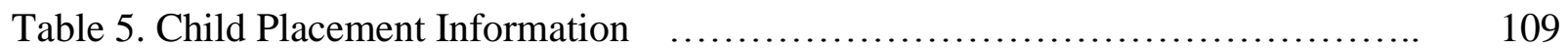

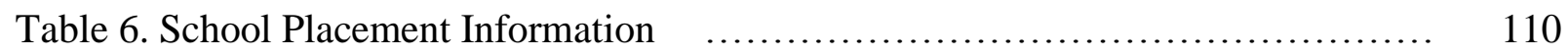

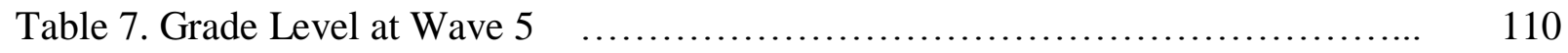

Table 8. Caregiver Demographics at Wave $1 \quad \ldots \ldots \ldots \ldots \ldots \ldots \ldots \ldots \ldots \ldots \ldots \ldots \ldots \ldots \ldots \ldots \ldots \ldots \ldots$

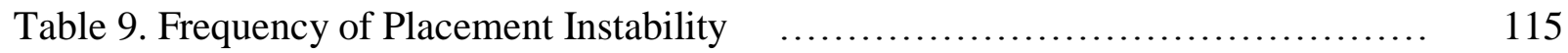

Table 10. Descriptive Statistics for Time-invariant Covariates from

Wave 1 and Distal Outcome at Wave $5 \quad \ldots \ldots \ldots \ldots . . . . .116$

Table 11. Comparison of Mean Values with Outliers Excluded $\quad \ldots \ldots \ldots \ldots \ldots \ldots \ldots \ldots . . . \ldots 117$

Table 12. Wave 1 Factor Loadings and $R^{2}$ for CFA Model of Emotion

Dysregulation

Table 13. Wave 3 Factor Loadings and $R^{2}$ for CFA Model of Emotion

Dysregulation

Table 14. Wave 4 Factor Loadings and $R^{2}$ for CFA Model of Emotion

Dysregulation

Table 15. Factor Loadings and $R^{2}$ for CFA Model of Emotion

Dysregulation across Waves 1, 3, and 4

Table 16. Correlation, Covariance, and Variance for Estimated Factor

Scores

Table 17. Point Estimates of the Second-Order Growth Model with

Dichotomous Indicators

Table 18. Model Fit Index for Wave 1 LCA Model 
Table 19. Model Fit Index for Wave 3 LCA Model

Table 20. Model Fit Index for Wave 4 LCA Model

Table 21. Cross-sectional Comparison of Class Counts \& Proportion of Children in Each Class

Table 22. Cross-sectional Comparison of Class Selection Probabilities

Table 23. Model Fit Index for LTA Model

Table 24. Transition Probabilities across Waves

Table 25. Odds Ratio for the Model Coefficients with

Gender as a Covariate Predicting Class Membership

Table 26. Class Counts and Proportions of Children within

Each Latent Class by Gender

Table 27. Class counts and proportions

Table 28. Odds Ratio for the Model Coefficients with Covariates

Predicting Class Membership

Table 29. Transition Probabilities for the Distal Outcome and

Unconditional LTA Models

Table 30. Latent Class Patterns across Waves 1, 3, and 4

Table 31. Mean and Standard Deviation for Wave 5 Academic

Achievement by Latent Class

Table A1. Within-PSU Sampling Domains for the NSCAW CPS

Sample

Table B1. Available Weights for Analyzing NSCAW Data

Tables C1 - C3. HHS Poverty Guidelines

Tables D1 - D3. Cross-tabulation for Poverty Estimation

Table E1. Missing Data Pattern Frequencies 


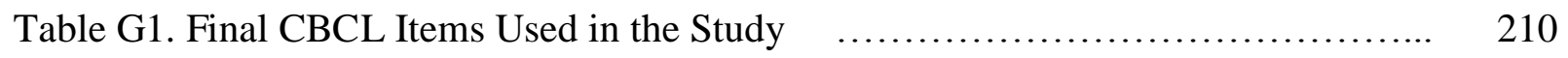

Table H1. Final SSRS Items Used in the Study $\quad \ldots \ldots \ldots \ldots \ldots \ldots \ldots \ldots \ldots \ldots \ldots \ldots \ldots \ldots \ldots \ldots \ldots \ldots$

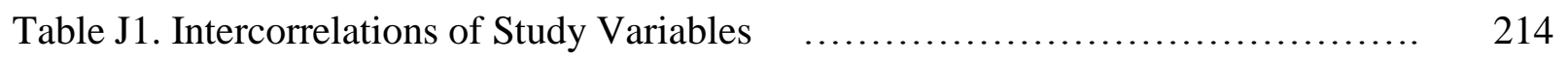

Table K1. Wave 1 Probability of Problem Behaviors for Latent

Classes 1 and 2

232 


\section{List of Figures}

Figure 1. Process-Person-Context-Time (PPCT) conceptual model. 7

Figure 2. Overlap of disciplines in addressing academic vulnerabilities of maltreated children. 11

Figure 3. Expanded conceptual model showing specific school readiness domains across time. 12 Figure 4. Bio-ecological model with interrelated systems that influence development over time.

Figure 5. Process model of emotion regulation ................................................................ 49

Figure 6. Standard structural diagram for a second-order latent growth model....................... 97

Figure 7. Second-order growth model with ordinal indicators. Jagged lines represent the link function that provides an underlying latent continuum transformation of the ordinal indicators.

Figure 8. Latent class model across 3 time points with first order effects.

Figure 9. Profiles for the 2-class model showing emotionally regulated and dysregulated maltreated children at wave 1 .

Figure 10. Profiles for the 2-class model showing emotionally regulated and dysregulated maltreated children at wave 3 .

Figure 11. Profiles for the 2-class model showing emotionally regulated and dysregulated maltreated children at wave 3 .

Figure 12. Proportions of each latent class at each wave.

Figure 13. Second-order effect model for latent transition analysis

Figure 14. Developmental patterns of emotion dysregulation across waves 1, 3, and 4.

Figure 15. Second-order LTA model with covariates included

Figure 16. Second-order LTA model with distal outcome included

Figure 17. Academic achievement at wave 5 for emotionally regulated vs. dysregulated latent classes. 


\section{Chapter 1: Introduction}

Academic achievement in the early school years is important for the long-term educational well-being of children with an early history of maltreatment. Unfortunately, the gap in academic achievement between this group of vulnerable children and their non-maltreated counterparts is increasing (Barrat \& Berliner, 2013; Stone, 2007). By elementary and high school, many of these children often fall behind their non-maltreated peers in measures of academic performance. These include relatively low scores on standardized tests (Coohey, Renner, Hua, Zhang, \& Whitney, 2011; Crozier \& Barth, 2005), below average grades (KendallTackett \& Eckenrode, 1996; Slade \& Wissow, 2007), maladaptive behaviors in the classroom (Snow, 2009; Zima, Bussing, Freeman, Yang, Belin, \& Forness, 2000), increased chances of grade level retention (Snow, 2009; Stone, 2007), increased rates of absenteeism (Leiter, 2007; Rouse \& Fantuzzo, 2009), and increased special education placement (Scarborough \& McCrae, 2010; Stone, 2007).

These poor academic outcomes have been associated with several individual-level and context-level factors. Sources of variation in individual-level factors may result from physiological dispositions such as temperament and cognitive abilities, whereas sources of variation in context-level factors may result from differences in socioeconomic status, educational attainment, neighborhood quality, or school quality. These individual-level and context-level factors may be associated in a way that affects propensity to experience maltreatment or that influences school achievement. Specifically, factors within the child include lower cognitive functioning, language delays, and behavior and emotion regulation problems (Crozier \& Barth, 2005; Eigsti \& Cicchetti, 2004; Maughan \& Cicchetti, 2002; Pears, Fisher, Bruce, Kim, \& Yoerger, 2010; Pears, Heywood, Kim, \& Fisher, 2011). With respect to these 
children's context or environment, frequent home and school movement, as well as less stimulating home environments, contribute to poor academic outcomes (Stacks \& Partridge, 2011; Stone, 2007).

Unfortunately, attempts to close the achievement gap at later ages can prove to be challenging for several reasons. First, later outcomes in academic achievement are dependent on performance in the early school years, with negative performance having cascading effects over time. Second, core developmental processes that are associated with these academic outcomes need to be further understood and addressed. In fact, the National Research Council and Institute of Medicine (NRC/IOM; 2000) treatise, "From Neurons to Neighborhoods", emphasized the importance of three domains from birth to age 5 that are important for children's success: selfregulation capabilities; communication and language; and social relationships. These domains are especially salient for children with a history of maltreatment given that experiencing compromised caregiving early in life has implications for children's language and socioemotional functioning over time (Eigsti \& Cicchetti, 2004; Maughan \& Cicchetti, 2002; Pears et al., 2010; Pears, et al., 2011). Third, our understanding of the academic challenges experienced by maltreated children is still very limited. These children experience multiple transitions in their lives that have implications for their academic outcomes (Altschuler, 1997; Yu, Day, \& Williams, 2002). For example, we need to further understand developmental transitions, school transitions between grade levels, and home transitions. Finally, attempts at understanding and delivering interventions for these children have rested on disciplines that have remained within their own silos. Addressing the educational needs of maltreated children requires an interdisciplinary perspective that includes developmental science, education, and child welfare. 
The goal for this study, therefore, was to draw from these perspectives in order to further understand the individual- and context-level factors that are associated with the development of preschool-aged maltreated children and their later academic achievement. More specifically, the developmental trajectory of emotion regulation development across 3 years since the involvement of Child Protective Services (CPS) was investigated. Additionally, this study explored how gender, language, placement stability, and caregiver relationships influenced development. Finally, a person-centered approach was employed in order to examine how specific profiles were related to academic achievement by later school age.

\section{Maltreatment in the Early Childhood Period}

In 2011 , there were a total of 675,569 substantiated victims of child abuse and neglect identified in the United States (Administration for Children and Families, 2012). This total number increases significantly to 6.2 million children when counting both substantiated and unsubstantiated referrals to Child Protective Services (CPS; Administration for Children and Families, 2012). The largest portion of referrals to CPS were classified as neglect (75\%), with physical abuse as the second largest (18\%), followed by sexual abuse (9\%). Preschool-aged children (i.e., 3-5 years old) comprise $23.4 \%$ of the victims referred to CPS, which makes them the second largest group second to infants and toddlers (Administration for Children and Families, 2012). Often, the well-being outcomes of this understudied group of children are overshadowed by research and interventions focused on infants and toddlers. This is unfortunate given that this group of children is entering the child welfare system at a time that is critical for their development of school readiness skills. Additionally, the children that enter the child welfare system during preschool displayed more developmental delays and less access and use of intervention services than other age groups (Stahmer, Leslie, Hurlburt, Barth, Webb, Landsverk, 
\& Zhang, 2005; Zimmer \& Panko, 2006). Finally, receiving the attention of CPS by 3-5 years of age means that these children may have been exposed to compromised caregiving environments for an extended period of time without access to additional support. This means that the negative consequences of maltreatment may be more pronounced.

The National Child Abuse and Neglect Data System (NCANDS; Administration for Children and Families 2011, p. 115) offered the following definition for maltreatment, accounting for state differences in maltreatment indicators:

"An act or failure to act by a parent, caregiver, or other person as defined under State law which results in physical abuse, neglect, medical neglect, sexual abuse, emotional abuse, or an act or failure to act which presents an imminent risk of harm to a child."

Evidence for examining how each subtype of maltreatment affects developmental outcome exists. However, given concerns about reliability and validity of maltreatment types when using the NSCAW study, a homogenous analysis of maltreatment type was conducted. That is, outcomes for children was examined broadly rather than disaggregated by maltreatment type. According to Cicchetti and Manly (1990), extreme care must be exercised in delineating maltreatment type.

\section{Theoretical Background and Conceptual Model}

Understanding the effects of maltreatment on children's core developmental processes requires multiple perspectives in order to tease apart the complexities in these children's lives. To this end, a framework that incorporates ideas from developmental science, early childhood education, and child welfare was adopted. Each perspective is covered briefly, along with a discussion of how they each contribute to our understanding of the developmental processes of 
maltreated children. The section concludes with an integrated view of these different perspectives, along with a final conceptual model for this study.

\section{Developmental Science}

According to Lerner, Theokas, and Bobek (2005), contemporary developmental science has transcended previous Cartesian philosophical ideas about the duality or split phenomenology of human development (e.g., nurture vs. nature). Instead, the prevailing philosophy views human development as involving mutually influential or bidirectional relationships between multiple layers of organization "from biology through individual and social functioning to societal, cultural, physical, ecological, and ultimately, historical levels of organization" (p. 3). Through these mutually influential relationships, change occurs. However, to understand whether the witnessed change is developmental in nature, certain assumptions about these changes have to be met. That is, these changes must be systematic, organized, successive. The successive characteristic of change implies that any later or distal developmental outcome can be understood as being fully or partially influenced by changes that occurred at an earlier time. Thus, growth and development can be understood as a systematic and organized set of changes that occur over time through the mutual interaction of the individual and his or her environment.

This notion of human development as shaped by dynamic and continuous interactions between biology and experience has been echoed in the National Research Council and Institute of Medicine (NRC/IOM; 2000) volume "From Neurons to Neighborhoods." These relationships are considered the building blocks of healthy development, which unfolds along pathways characterized by continuities, discontinuities, and transitions. Further, development is shaped by the interplay among sources of vulnerability and resilience within the individual, as well as between the individual and the environment. Finally, timing of early experiences is important in 
shaping development, but a child remains vulnerable to risks and open to protective influences throughout the life course. This course of development, then, can be altered by interventions that change this risk/protective factor balance (NRC/IOM, 2000).

To capture the systems of interrelated changes, a Process-Person-Context-Time (PPCT) model was employed by this study (see Figure 1). Within this model, we can see that early maltreatment experience has a direct impact on academic achievement for children at a later time (e.g., school age or adolescence). However, in order to further unpack this relationship, the model incorporates mechanisms that may explain this development across a continuum. Specific to the current study, core developmental processes within the individual were examined, particularly regarding their contribution to later academic outcomes in children with a history of maltreatment. Additionally, this model assumes that context plays a role in how children's developmental processes emerge. As such, this model incorporates the role of these context-level factors in children's development, which in turn affects later academic outcomes.

Bio-ecological theory was the main PPCT model used for this study because it incorporates complicated and dynamic systems that lend itself to theory or hypothesis testing (Bronfenbrenner \& Morris, 2006). This theory proposed that four interrelated and dynamic principles or properties are involved in promoting growth and development: proximal processes, person, context, and time. Central to this study are the dynamic processes between person-level and context-level principles that, over time, influence specific developmental outcomes. Indeed, these proximal processes have been implicated as the primary mechanism in promoting change and development. Often, these processes are most influential early in a child's life because it is through these interactions that human beings organize their set of cognitive, emotional, physiological, and behavioral responses to their world (Thelen \& Smith, 2006). 


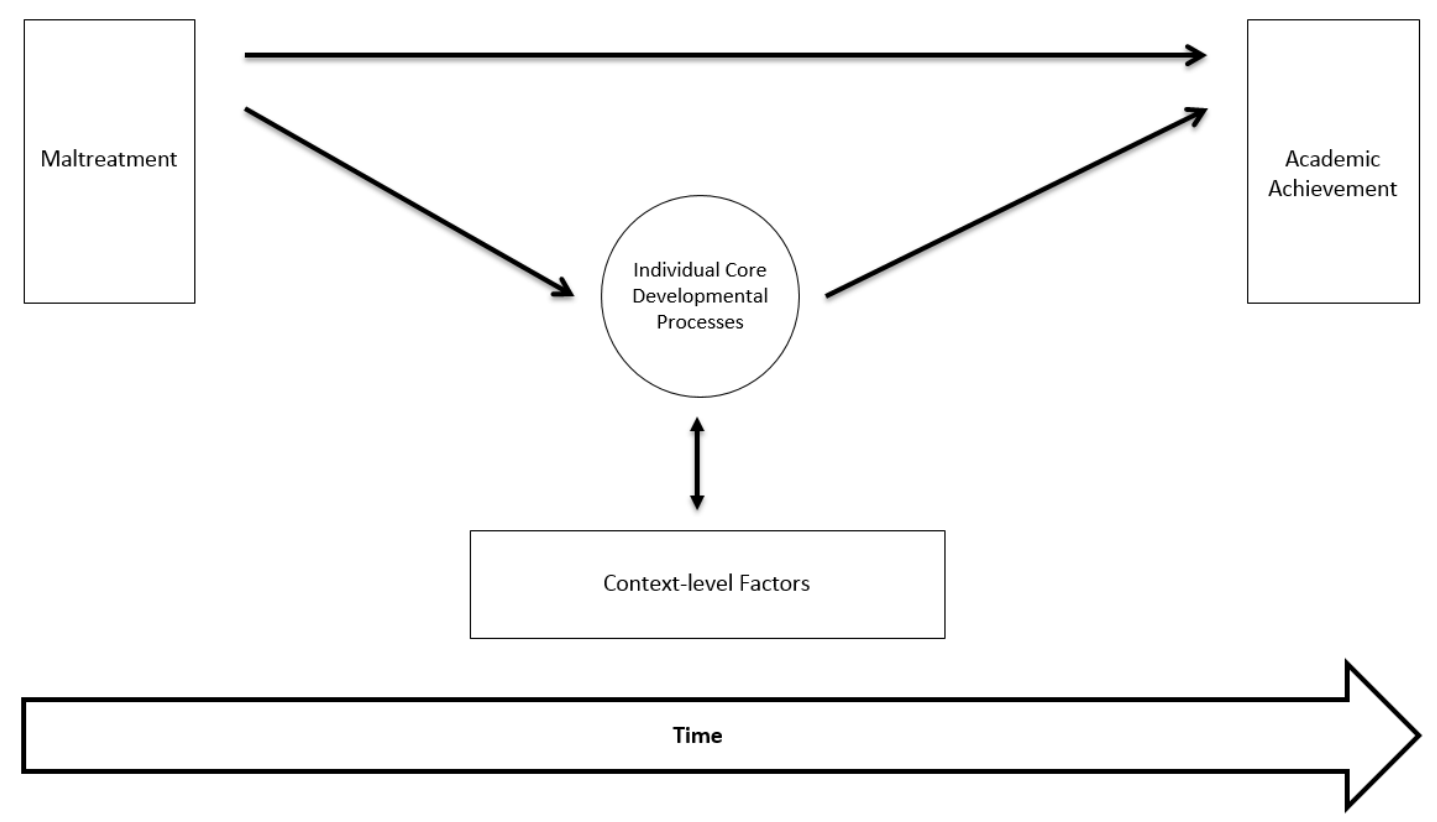

Figure 1. Process-Person-Context-Time (PPCT) conceptual model.

Thus, for maltreated children, it is necessary to understand their unique caregiving context, which includes child abuse and/or neglect as well as their experiences after entering the child welfare system at a young age. Contact with Child Protective Services (CPS) introduces specific contextual experiences such as disruption in living situations, introduction of foster care, and variation in caregiving quality. This, in turn, affects the development of regulatory skills and language that may be specific to maltreated children.

\section{Early Childhood Education}

Interest in the academic achievement of young children necessitates the perspective of the field of early childhood education (ECE). Specifically, ECE scholars assert that children need to be prepared for entry into formal schooling (i.e., kindergarten), and that successfully navigating this transition has implications for later academic achievement (e.g., Halle, Hair, Wandner, \& Chien, 2012). In order to prepare children for this transition into formal schooling, the National 
Education Goals Panel identified 5 developmental domains necessary to promote school readiness (Kagan, Moore, \& Bredekamp, 1995). These are physical well-being and motor development, socioemotional development, approaches to learning, cognitive and general knowledge, and language. Further, the National Research Council (NRC; 2001) argues that the home and school environments are important contextual domains that also influence children's learning. Pianta (2007) added that incorporating early childhood education and development science perspectives has influenced educators to acknowledge that context is important in young children's development and that early school grades are critical for later school success. School readiness, therefore, is a multidimensional concept that is important in supporting children's transition into kindergarten and early elementary school.

In the NRC/IOM (2000) study, "From Neurons to Neighborhoods," the authors recommended that:

"Resources on a par with those focused on literacy and numerical skills should be devoted to translating the knowledge base on young children's emotional, regulatory, and social development into effective (teaching) strategies...” (p. 5).

The authors further recommended that school readiness initiatives should be judged based on their effectiveness in improving achievement outcomes. In addition, these initiatives should also provide opportunities to reduce disparities at school entry for young children with different backgrounds (e.g., young maltreated children). Two particular domains related to school readiness develop between birth and age 5: socioemotional functioning and language skills. The authors noted that during this time, children negotiate the transition from externally guided regulation to self-regulation. This includes learning how to regulate emotions, behavior, and attention. In addition, it is also during this time period that social interactions occur and children 
learn to relate with others and form friendships. Finally, it is in the period of birth to 5 that children acquire the foundational skills that promote communication and learning, which includes early development of language, reasoning, and problem solving.

With this perspective in mind, it is possible to envelop the core developmental processes of emotion regulation and language within the concept of school readiness. From a developmental standpoint, the functional organization of emotion regulation and the development of language increase at an accelerated rate during early childhood compared to earlier ages. Enhancing the development of these two processes are consistent with the promotion of school readiness domains during preschool, as one of the goals of early childhood educators. Thus, there appears to be a natural fit in integrating the early childhood education perspective with developmental science, specifically regarding the focus on specific school readiness domains.

\section{Child Welfare}

The child welfare field has emphasized that, in addition to safety and permanency, child well-being is a critical goal of service delivery to maltreated children (Administration for Children and Families, 2011). However, tensions exist in how to incorporate these three outcomes within the child welfare service delivery system. To address this underlying tension, Wulczyn, Barth, Yuan, Jones Harden, and Landsverk (2005) proposed a framework to understand well-being as a "relative estimate of how a child is doing given certain assumptions and prior knowledge about his or her developmental path or trajectory" (p.24). They added that when considering developmental outcomes, it is important to take into account that Child Protective Services (CPS) is part of the ecological context that influences this trajectory. Consideration of age or developmental periods such as infancy, early childhood, middle 
childhood, and adolescence was proposed as a useful heuristic. This is particularly important when examining age-specific outcomes of maltreatment and CPS involvement.

The authors offered two concepts to bridge human development, maltreatment, and placement: onset, which refers to the occurrence of an event; and timing, which places events across the developmental trajectory that began at the point of onset. In other words, well-being can be understood as having an initial state as well as movement along a developmental trajectory. Adopting this viewpoint allows one to place maltreatment experiences within a developmental perspective. Additionally, interventions designed to impact well-being require knowledge about initial developmental levels, as well as the growth trajectory of that child based on those initial levels (Wulczyn et al., 2005).

Given that school readiness skills develop during preschool, it is important to understand what impact entry into the child welfare system during this time may have on developmental trajectories. As mentioned earlier, it is during early childhood that the core developmental processes of emotion regulation and language mature at a rapid pace. As with other developmental periods, the quality and stability of caregiving is of great importance in promoting positive developmental outcomes during the early childhood period. However, children's involvement with child welfare during the preschool period precipitates instability across multiple contexts. Children's living arrangements are often disrupted such that they may be placed in foster care or relative care, and may be subsequently returned home. Additionally, for children in formal preschool or school arrangements, school instability disrupts the relationshipbuilding between student and teacher, and children's potential mastery of instructional material. Because of these instabilities, the trajectory of core developmental processes, and thus school 
readiness, may be negatively impacted. This in turn places maltreated children at a disadvantage when they transition from preschool to kindergarten and early elementary school.

\section{Integration}

The prevalence of child maltreatment in the United States highlights the importance of learning about, and intervening with, children who will potentially experience academic difficulties. However, the prevention of academic failure for the large number of children experiencing early maltreatment requires a deeper understanding of the developmental outcomes of these children from the perspectives of developmental science, early childhood education, and child welfare (see Figure 2).

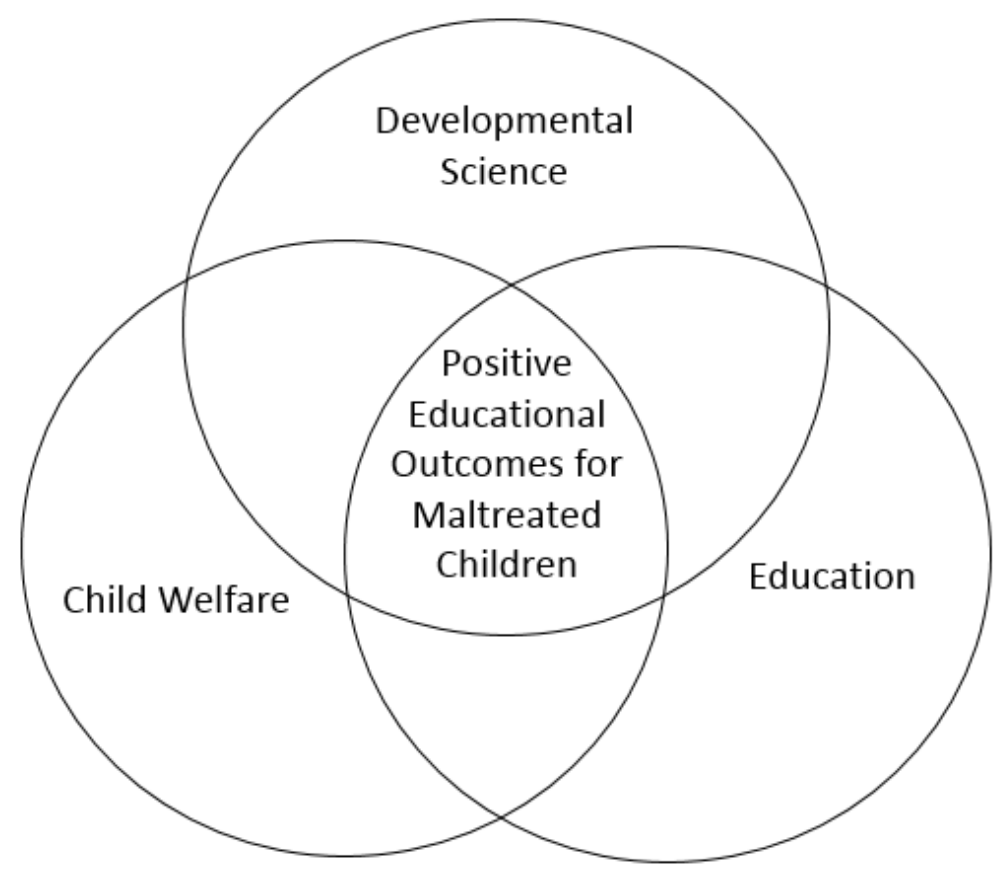

Figure 2. Overlap of disciplines in addressing academic vulnerabilities of maltreated children

Across these disciplines, there are several overlapping themes that could be incorporated into an integrated conceptual framework. First, a focus on growth and development across childhood points to sensitive periods, particularly during early childhood, in the development of emotion regulation and language. These, in turn, have implications for later academic 
achievement. Second, the quality of interaction between the child and his or her environment is important in influencing developmental outcomes. Specifically for children with a history of maltreatment, the quality and stability of interaction between caregiver and child are important in promoting better regulatory and language skills. Finally, transition points are important to consider when studying maltreated children's developmental outcomes. Specifically, maltreated children are faced with multiple transition points and experiences: developmentally (toddlerhood to preschool to school age), academically (preschool to kindergarten to early elementary), and home situations (foster placement, placement with relatives, returning home). Successfully navigating these transitions has implications for later academic achievement. The full conceptual model, which includes these concepts, can be seen in Figure 3.

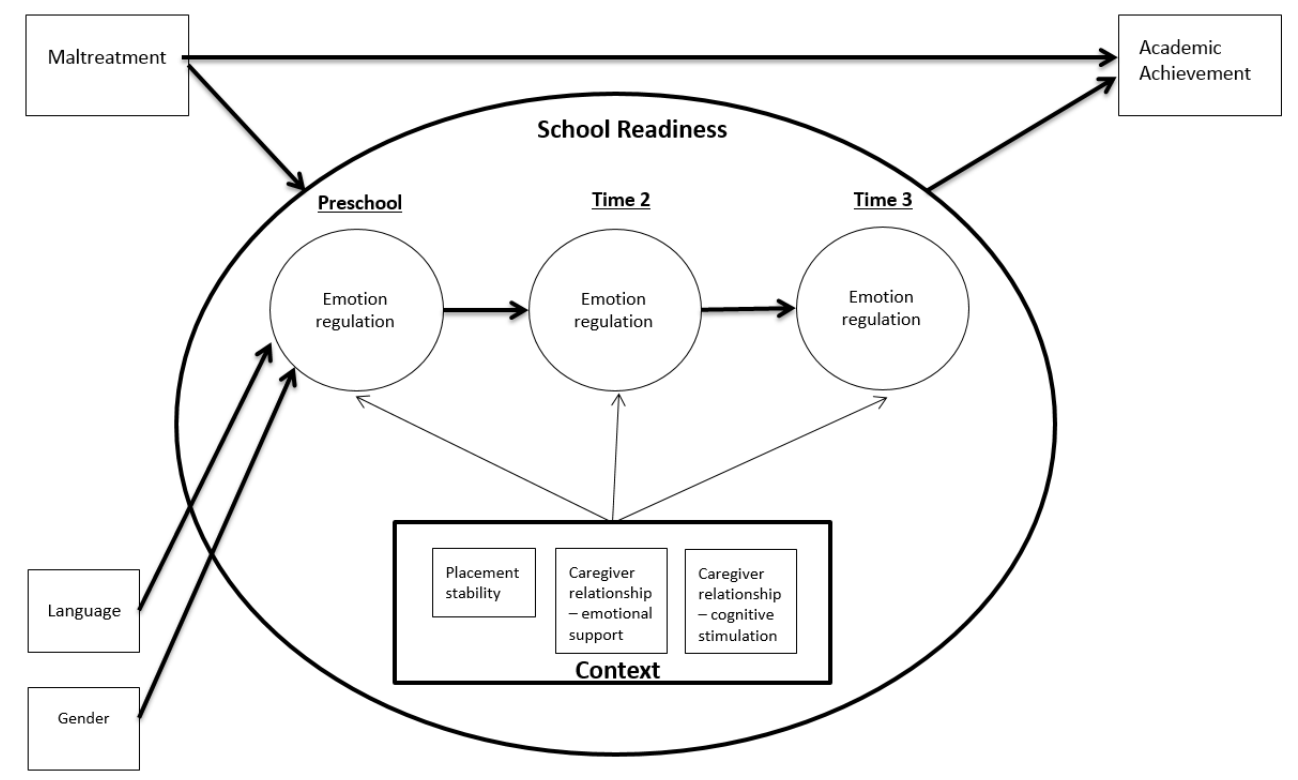

Figure 3. Expanded conceptual model showing specific school readiness domains across time.

\section{Overview of the Literature}

Indicators and measures of academic achievement in non-maltreated children have often included reading and math standardized test scores, student suspension and expulsion rates, 
public reports on school success in impacting student achievement in grades 3 through 8 and in high school, incidents of violence and substance abuse among students, and dropout rates (Center for the Study of Social Policy, 2003). Studies that have examined at-risk, non-maltreated families suggest that multiple pathways exist that explain variations in these academic achievement outcomes for young children. For example, emotion regulation and language processes (Cristofaro \& Tamis-LeMonda, 2011; Kim, Nordling, Yoon, Boldt, \& Kochanska, 2013; Sektnan, McClelland, Acock, \& Morrison, 2010; Song, Spier, \& Tamis-LeMonda, 2014), along with context-level factors such as parent-child relationships and neighborhood (Cristofaro \& Tamis-LeMonda, 2011; Mistry, Benner, Biesanz, Clark, \& Howes, 2010; Pan, Rowe, Spier, \& Tamis-LeMonda, 2004), have been implicated in predicting academic performance. More specifically, these school readiness skills need to be developed during early childhood in order for children to succeed academically during the elementary school years and beyond (Cristofaro \& Tamis-LeMonda, 2011; McWayne, Cheung, Wright, \& Hahs-Vaughn, 2012; Mistry et al., 2010).

Unfortunately, early maltreatment may provide additional risks for school readiness and later academic achievement outcomes. As mentioned at the start of this chapter, children experience additional compromised outcomes specific to maltreatment. This suggests that the pathways to later academic outcomes for maltreated children may be especially important. That is, core developmental processes (i.e., emotion regulation and language) and caregiving contexts may have differing effects for children with a history of maltreatment, which will be covered next. 


\section{Emotion Regulation}

Emotion regulation can be understood as a system of cognitive, behavioral, and emotional aspects of functioning that enables effortful control or modulation of emotions and behaviors, which facilitates the interaction between an individual and his or her environment in order to attain specific goals (Blair \& Raver, 2012; Cole, Marin, \& Dennis, 2004; Thompson, 1994). With this definition in mind, it is important to note that emotion regulation is a requisite skill for success in the academic setting, in that children modulate their dominant response in favor of attending to class content, interacting appropriately with peers and teachers, and managing frustration with challenging materials. Indeed, Pears, Fisher, Bruce, Kim, and Yoerger (2010) found that inhibitory control (a cognitive component of emotion regulation) at preschool fully mediated the effects of early maltreatment on academic competence at school age. The ability to regulate emotions can also support school adjustment and learning, particularly when intense emotions are managed and attentional resources are focused on the task at hand (Raver, Jones, Li-Grining, Zhai, Bub, \& Pressler, 2011).

Unfortunately, children with a history of maltreatment often display dysregulated emotional responses across home and school settings. For example, maltreated children exhibit attentional bias for negative stimuli, making it difficult to disengage from highly emotional distractions in order to focus on academic tasks (Maughan \& Cicchetti, 2002). Additionally, maltreated children exhibit overuse of certain behavioral responses as regulatory mechanisms. These include externalizing or internalizing behaviors (Kim and Cicchetti, 2010; Schelble, Franks, \& Miller, 2010). 


\section{Language}

The ability to comprehend and express language is central to academic success as it is the main vehicle by which class content is communicated. Additionally, it is through verbal instructions that information can be encoded into long-term memory and retrieved for later use. The ability to verbally communicate is also important for peer interactions in the school setting, as well as to communicate with adults (e.g., teachers, caregivers, school counselors).

Additionally, for children and adults, language can serve as a means to communicate how they feel and thus serves a regulatory function. Unfortunately, evidence exists of compromised receptive and expressive language abilities for children with a history of maltreatment (Eigsti \& Cichetti, 2004; Pears, Heywood, Kim, \& Fisher, 2011). More specifically, early maltreatment has been shown to predict less advanced vocabulary and lower production of language (Eigsti \& Cichetti, 2004), as well as decreased phonological awareness, which places maltreated children at risk for learning problems (Pears et al., 2011).

These core developmental processes appear to work in tandem to influence children's academic outcomes (Raver, Garner, \& Smith-Donald, 2007). More specifically, these processes appear to be interrelated in that compromised functioning in one or more domains may have negative cascading effects on the other domains (Cole, Armstrong, \& Pemberton, 2010). For example, poorly regulated emotions, such as anxiety, may decrease cognitive functioning (e.g., memory encoding and attentional resources), leading to decreased language comprehension. What follows next is a brief review of context-related factors that affect the development of these core developmental processes, as well as academic outcomes. 


\section{Placement Stability}

Children and families often become involved with Child Protective Services (CPS) when allegations about maltreatment are made. Investigations could result in substantiated findings, meaning that evidence of maltreatment has been documented. Based on the degree of safety and/or severity of the maltreatment behavior, several options are available from which CPS investigators choose. These may include removal from the child's birth family home and placement into foster care or maintaining the birth family placement with the addition of supportive services. Regardless of the placement decision, implications for stability in these children's living environments must be considered. According to bio-ecological theory, proximal interactions need to occur on a regular and consistent manner between the child and a consistent caregiver in order to promote change and development (Bronfenbrenner \& Morris, 2006).

Unfortunately, involvement with CPS often entails frequent changes in a child's home environment. These instabilities could be related to removal from their birth family homes, placement in foster homes, or changes in foster placement. Decreasing the duration that children have to interact with their parents or caregivers severely limits children's exposure to consistent caregiving, which is needed in order to promote the development of emotion regulation, cognitive, and language processes. In fact, it has been shown that increased instability in a child's home or placement is associated with negative developmental outcomes such as language, cognitive functioning, and behavior problems (Jones Harden, 2004; Lloyd \& Barth, 2011; Ryan \& Testa, 2005).

\section{Parent/Caregiver-Child Relationship}

Based on bio-ecological tenets, the parent-child relationship is the primary mechanism for change in promoting developmental outcomes for children, given that it represents proximal 
processes that link the child's microsystem (i.e., home environment) with each child's individual capacities. In this case, relationship qualities related to language and cognitive stimulation will promote language development, whereas emotional supportiveness and sensitivity will promote emotion regulation development. There is also evidence that emotional support, especially in early childhood, promotes language and cognitive development as well (Jones Harden \& Whittaker, 2011).

Unfortunately, onset and timing of maltreatment, as well as involvement with CPS, place these children at risk for experiencing compromised caregiving and unstable living arrangements. It is therefore important to understand the contribution of the caregiving context that may promote positive development in these core developmental processes, with implications for later academic achievement. According to Jones Harden and Whittaker (2011), higher cognitive stimulation and emotional support, especially in the early home environment, predicted higher cognitive and language scores, as well as decreased behavioral problems by the time maltreated children reached preschool. This indicates the important role that proximal processes play in the promotion of core developmental processes.

\section{Gender}

Consideration of gender differences in academic outcomes and developmental processes is important when trying to understand the person-level factors involved in differential outcomes. Understanding these differences could potentially provide important information for adapting strategies based on gender differences. Predominant gender differences in developmental processes have been linked with selection of emotion regulation strategies wherein boys select more externalizing (i.e., aggressive) strategies whereas girls selected more internalizing (i.e., withdrawal) strategies (Keenan \& Shaw, 1997). Additionally, language delays have been shown 
to be more predominant in boys than girls (Scarborough \& McCrae, 2010). With regard to academic outcomes, boys, more than girls, are more likely to receive special education services (Scarborough \& McCrae, 2010).

Taken together, these studies underscore that maltreated children appear to have compromised developmental processes and contexts, which can ultimately impact their academic achievement. However, several gaps still exist in understanding the interrelatedness of these core developmental processes, context, and later academic achievement. First, there are relatively few studies that examine growth patterns of core developmental processes in maltreated children spanning 3 years since first contact with CPS during the preschool period. Second, context-level factors such as placement status, placement and school stability, and caregiver/parent relationship should be examined in a way that would elucidate their potential role in influencing the development of emotion regulation. Finally, even fewer studies have looked at socioemotional school readiness skills of maltreated children since first contact with CPS at preschool, and how this impacts later school achievement.

\section{Specific Aims and Research Questions}

Given the gaps in the empirical literature on academic outcomes among maltreated children and what factors predict these outcomes, this study had several specific aims. The first aim was to understand the long-term effects of onset and timing of first contact with CPS on maltreated children's school readiness skills. This was done by examining growth curves and latent class transitions of emotion regulation patterns across first contact with CPS during the preschool period and 3 years after. The second aim was to understand how gender, language, placement stability and caregiver relationships affect development of maltreated children's emotion regulation patterns. More importantly, children involved with CPS may have different 
contextual experiences across this developmental trajectory. A final aim of the study was to understand how specific emotion regulation patterns affected later school achievement for a sample of young maltreated children. In addition to these research aims, this study employed person-oriented methods in order to identify groups or subgroups of similar developmental patterns exhibited by children in this sample. More specifically, this study was guided by the following research questions:

1) What are the growth trajectory patterns of emotion regulation development for young maltreated children across 3 years since time of first contact with Child Protective Services?

2) Is there heterogeneity in young maltreated children's display of emotion regulation and do they exhibit stability and change in these developmental patterns across time?

3) To what extent do emotion regulation class memberships differ by gender, language, placement stability, caregiver emotional support, and caregiver cognitive stimulation?

4) What are the different pathways in which emotion regulation predicts later academic achievement for young maltreated children who had contact with CPS during the preschool period?

In order to answer these questions, the National Survey of Child and Adolescent WellBeing (NSCAW I) restricted release data was used. NSCAW is a nationally representative, longitudinal survey of children and families who were investigated by CPS. The NSCAW study was a fixed panel design with 5 waves of data collection from 1999 through 2007 . There were 5,501 children in the CPS sample, with an age range of birth to 14 years. The study oversampled infants and sexual abuse cases in order to ensure ample statistical power. However, sample weights were provided to maintain nationally representative proportions. A subsample of 
preschool-aged children $(N=834)$ was selected for this study and followed across waves $1,3,4$, and 5.

Data analysis proceeded in a principled step-wise manner, which began with descriptive statistics on the variables of interest presented in the conceptual model above. Then, specific models were employed for each of the questions stated. First, latent growth curve models were used to answer question 1. Next, person-centered methods such as latent class analysis (LCA) and latent transition analysis (LTA) were employed. Specifically, LCA was employed as a measurement model to enumerate the number of latent classes that exist in the emotion regulation patterns of young maltreated children. Then, an LTA model was selected in order to examine developmental patterns in emotion regulation/dysregulation across time. A conditional LTA model was then employed to answer the third research question. In this step, auxiliary variables (i.e., gender, language, placement stability, caregiver cognitive stimulation, and caregiver emotional support) were included in the model in order to predict class membership in each of the latent classes derived from answering the second research question. Finally, later school achievement was examined as a distal outcome predicted by the emotion regulation latent class at wave 4.

\section{Definition of Key Terms}

Maltreatment: An act or failure to act by a parent, caregiver, or other person as defined under State law that results in physical abuse, neglect, medical neglect, sexual abuse, emotional abuse, or an act or failure to act which presents an imminent risk of harm to a child (Administration for Children and Families, 2012). 
School readiness: A multidimensional concept that is important in supporting children's transition into kindergarten and early elementary school ensuring readiness to learn. These dimensions include (1) physical well-being and motor development, (2) social and emotional development, (3) approaches toward learning, (4) language development, and (5) cognition and general knowledge. Contextual considerations such as the home and school environments are integrated within each of these domains (Kagan et al., 1995).

Academic achievement: Indicators and measures of achievement often include reading and math scores, student suspension and expulsion rates, public reports on school success at impacting student achievement in grades 3 through 8 and in high school, incidents of violence and substance abuse among students, and dropout rates (Center for the Study of Social Policy, 2003). Operationalization of academic achievement for this study, however, focused specifically on verbal and quantitative achievement scores.

Emotion regulation: Emotion regulation is a system of cognitive, behavioral, and emotional aspects of functioning that enables effortful control or modulation of emotions and behaviors, which facilitates the interaction between an individual and his or her environment in order to attain specific goals (Blair \& Raver, 2012; Cole, Marin, \& Dennis, 2004; Thompson, 1994).

Language: Language is a tool for communication to express thoughts and feelings to others and to receive and interpret communications from other people. Language plays an equally crucial role in providing symbols for concepts, which is fundamental for cognitive development. (Kagan et al., 1995). 
Placement stability: Children in the child welfare system experience multiple placement transitions such as placement outside of the home, between foster placements, and reunification. These placement disruptions or lack of stability result in negative developmental outcomes for children due to the lack of stable proximal processes represented by quality caregiving (Jones Harden \& Whittaker, 2011). For this study, placement stability was defined and measured as a new placement for one week or longer in a different household and/or with a new caregiver (Casanueva, Dozier, Tueller, Dolan, Smith, Bruce Webb, Westbrook, \& Jones Harden, 2014).

Caregiver relationship(cognitive/language stimulation): Caregiving behaviors that represent proximal processes between a child and a caregiver, which may promote positive or negative developmental outcomes. Positive, stable, and consistent cognitive stimulation can help promote language development and serve as a protective factor for maltreated children (Jones Harden \& Whittaker, 2011).

Caregiver relationship (emotional support): Similar to cognitive/language stimulation, emotionally supportive caregiving behaviors are proximal processes that promote positive or negative developmental outcomes. Positive, stable, and consistent emotional support can help promote socioemotional and language development, as well as serve as a protective factor for maltreated children (Jones Harden \& Whittaker, 2011). 


\section{Chapter 2: Review of the Literature}

Early maltreatment experiences have been associated with a host of deleterious effects on academic achievement by later school age (Rouse \& Fantuzzo, 2009; for a review, see Stone, 2007). However, ameliorating these poor academic outcomes during the late elementary years may prove to be challenging due to the cascading effects of earlier risk factors experienced by children with a history of maltreatment. Additionally, the pathway between maltreatment and academic outcomes is often not captured within a single trajectory. That is, multiple pathways may be created by the role of different school readiness abilities, stability of the home, and differing qualities in the caregiving environment. These intra-individual and contextual factors affecting maltreated children warrant further examination.

This chapter examines the extant research on the academic outcomes of children who experience early maltreatment. This section begins with a definition of maltreatment and description of the prevalence/incidence of maltreatment among U.S. children. Next, a detailed discussion of the conceptual framework is presented, which integrates the perspectives of developmental science, education science, and child welfare. This is followed by a review of the existing data on school readiness during early childhood broadly, then as it relates to maltreatment specifically. Next is an overview of empirical literature on academic outcomes specific to maltreated children, spanning elementary age through adolescence. The final section is a review of evidence linking individual and contextual factors with outcomes for maltreated children.

\section{Definition of Maltreatment}

A recent report by the Institute of Medicine and National Research Council (IOM/NRC, 2014) provided a detailed description of the definitional issues with respect to how child 
maltreatment is defined across multiple sources. The authors of the report asserted that variations in definitions of abuse and neglect may prove to be problematic for researchers if the research goals or data do not match the construct of maltreatment pertinent to the study. Several definitions of maltreatment are presented, which vary based on the perspectives of federal or state law, trend analyses, or research. Definition employed in this study is then provided.

Foremost to these definitional issues has been the use of federal legislation that allows states to adopt a minimum definition, while providing autonomy in specific standards. According to Section 3 of the Child Abuse Prevention and Treatment Act of 2010 (CAPTA), child abuse and neglect is defined as:

"Any recent act or set of acts or failure to act on the part of a parent or caretaker, which results in death, serious physical or emotional harm, sexual abuse or exploitation, or an act or failure to act, which presents an imminent risk of serious harm."

Across multiple states, maltreatment is often divided into 4 broad categories: physical abuse, neglect, sexual abuse, and emotional or psychological abuse. However, provision of a federal mandate that allows for variation in definitional standards across states presents a problem from the research and practice perspectives. In fact, problems with uniform definitions and common language can be seen as a result of variation in state adoption rates of CAPTA and differences in how each state defines maltreatment (Feely, Hayes, Mienko, Panlilio, \& Miyamoto, 2015).

Another source for defining maltreatment comes from the U. S. Department of Health and Human Services, Administration for Children and Families (ACF, 2011) using the National Child Abuse and Neglect Data System (NCANDS), which collects maltreatment reports from all states. NCANDS provides the following as a uniform definition across states: 
"An act or failure to act by a parent, caregiver, or other person as defined under State law that results in physical abuse, neglect, medical neglect, sexual abuse, emotional abuse, or an act or failure to act which presents an imminent risk of harm to a child."

This expanded definition allows for the capture of definitional variations in state laws when collecting data on incidence rates of maltreatment.

In addition to the definitions provided above, the National Incidence Study (NIS -4; Sedlak, Mettenburg, Basena, Petta, McPherson, Greene, \& Li, 2010) expanded upon the definitions reported above: a harm standard and an endangerment standard. The harm standard is used when children have shown demonstrable harm resulting from abuse and neglect, whereas the endangerment standard is more lenient and used when children have not yet been harmed under any circumstances. Selection of one standard over the other has implications for reported maltreatment rates. For example, using the harm standard, national data document that 1.25 million children are victims of abuse and neglect; using an endangerment standard raises this number to 3 million (IOM/NRC, 2014).

The Maltreatment Classification Scheme (MCS), developed by Barnett, Manly, and Cicchetti (1993), is another widely used method in studies which aim to define maltreatment subtypes. The MCS was devised to examine information from Child Protective Services (CPS) records with the goal of integrating multiple informants and multiple sources of information within a developmental psychopathology framework. The impact of maltreatment on children's development can be captured when using this framework (Manly, 2005). The MCS includes operational definitions for each type of maltreatment, along with coding schemes for each subtype and frequency of co-occurrence of each subtype. Additional dimensions captured by the MCS include timing of maltreatment (i.e., age of onset, frequency, chronicity, developmental 
period), relationship of perpetrators, occurrence of separations and placements, and severity of maltreatment incidents (Manly, 2005).

From a public health research perspective, the Centers for Disease Control and Prevention $(\mathrm{CDC}, 2008)$ provides a uniform definition in order to monitor incidence over time and detection of trends related to maltreatment. According to the CDC, childhood maltreatment is, "any act or series of acts of commission or omission by a parent or other caregiver that result in harm, potential for harm, or threat of harm to a child" (p. 11). Acts of commission, or abuse, were further defined as words or overt actions that cause harm, potential harm, or threat of harm. The types of maltreatment included within this category are physical, sexual, and psychological abuse. Acts of omission, or neglect, were defined as the failure to provide for the child's basic physical, emotional, or educational needs or to protect the child from harm or potential harm. Types of neglect include failure to provide (subtypes include physical, emotional, medical/dental, and educational neglect) and failure to supervise (subtypes include inadequate supervision and exposure to violent environments). According to the CDC definition, these acts have to be deliberate and intentional. However, the resulting harm to the child takes precedence over considerations of the intentionality of maltreatment.

Cicchetti and Manly (1990) advocated for delineating subtypes of maltreatment as outlined in the MCS. In addition, they recommended that variances in severity, chronicity, and age of onset be identified in order to more accurately assess etiologies and developmental consequences. However, the authors cautioned about the challenges in making the distinctions across maltreatment subtypes. Challenges with attaining accurate information were due to reliability of reporters (e.g., parents who are subjects of the investigation), difficulty with access to records (e.g., confidentiality), accuracy of recorded information, and variability across 
jurisdictions. The authors added that even if an exhaustive compilation of information was gathered, it would still be difficult to distinguish among the types of maltreatment. According to the authors, because most maltreated children experience more than one subtype of maltreatment, extreme care must be exercised in searching for "pure" maltreatment subtypes (Cicchetti \& Manly, 1990, p. 96). They argued for the use of total number of different types (i.e., incidence of type), use of a reliable and valid classification system (e.g., MCS), or use of systematic means of assessing types of maltreatment. Given concerns about reliability and validity of maltreatment types when using the NSCAW study, a homogenous analysis of maltreatment type was conducted.

This study employed the NCANDS definition of maltreatment for the following reasons. First, the NSCAW study employed a complex sampling design that included children and families from multiple states that employed different definitions of maltreatment. As stated earlier, the NDCANDS definition was developed in order to account for definitional variations. Second, this study examined the emotion regulation, language, and academic achievement of young maltreated children overall, rather than by maltreatment type. Next, the NSCAW Data File User's Manual (DFUM, 2008) specifically outlined procedures used in mapping the study data to definitions from NCANDS, making this a more valid definition for the study. Finally, given that maltreated children often experience multiple types of abuse, imposing subtypes in the analyses may not provide accurate information on the causal link between maltreatment type and child well-being outcome.

\section{Prevalence/Incidence of Maltreatment}

Despite the definitional variations noted above, prior research has documented the prevalence (i.e., overall number) and incidence (i.e., rate of increase in the population) of child 
maltreatment across the United States. The National Incidence Studies (NIS-4) employed a nationally representative design to estimate the number of abused and neglected children in the United States who come to the attention of community professionals. The NIS used maltreatment report data on abused and neglected children who have had contact with Child Protective Services (CPS). This included CPS referrals that have been "screened in" for investigation and those who have been "screened out" or dismissed. There have been four cycles of the report with the NIS-4 being the most recent one. Based on the NIS-4 report, there has been a dramatic decrease in the incidence rate of maltreatment based on the overall Harm Standard when compared to the NIS-3 in 1993, which amounts to a $26 \%$ decline per 1,000 children in the population (Sedlak et al., 2010).

Although there was an overall decrease in incidence, acts of maltreatment remain prevalent. In 2011, there were 9.1 victims of maltreatment per 1,000 children in the population, which added up to 676,569 substantiated victims of child abuse and neglect. Regardless of substantiation, however, there were 6.2 million children referred to CPS, highlighting the prevalence of maltreatment or risk of maltreatment in the population. Of these children referred to CPS, over $75 \%$ were classified as neglect, $18 \%$ as physical abuse, and $9 \%$ as sexual abuse. Girls accounted for $51.2 \%$ of the victims, while boys were $48.5 \%$. The highest rates of maltreatment reports occurred among infants and toddlers under 2 years old. Children aged 3-5 (i.e., preschoolers) comprised $23.4 \%$ of the victims reported to CPS, making them the second largest group of maltreatment victims next to infants and toddlers (ACF, 2012).

Given the high incidence and ongoing prevalence of this problem, research that examines the impact of maltreatment on child outcomes is critical. Although there has been substantial research on maltreated children's physical and mental health outcomes, fewer studies have 
addressed their academic achievement. Even less attention has been placed on the school readiness of young maltreated children. Thus, this review focused on the school readiness and later academic achievement of children with a history of maltreatment. Early school readiness and positive academic outcomes are important for facilitating maltreated children's achievement of high school graduation and later employment for successful independence (Vacca, 2008). Therefore, understanding the developmental pathways from maltreatment to negative academic outcomes has the potential to inform policy and practice to address this phenomenon.

\section{Theoretical Framework}

The strength of developmental science lies in the discipline's ability to transcend a unidimensional expression of an individual's development. Thus, development is embedded within a set of variables derived from multiple layers of organization, and is conceptualized as resulting from the dynamic relations among the different variables across these multiple layers (Lerner, Theokas, \& Bobek, 2005). Further, assumptions about change and development are necessary to the study of human growth. According to Lerner et al. (2005), development refers to change, though this term is not equivalent to development. Change is often a result of development, but not all changes are developmental in nature. According to the authors, changes must be systematic and organized in order to be considered developmental. Additionally, organized and systematic changes must be successive; changes seen at a later time are wholly or partly influenced by changes from an earlier time. The variability and probability of these later changes are limited by the earlier occurrences. In other words, human development is shaped by the dynamic and continuous interactions between the person, his/her experiences, and the multiple contexts in which he/she resides (NRC/IOM, 2000). 
Bio-ecological theory (Bronfenbrenner \& Morris, 2006) provides an overarching frame that can model development based on the assumptions delineated above. As this theory postulates, the inextricable link between the developing child and the environment allows for a richer understanding of the academic achievement of maltreated children. In addition, this theory provides flexibility in incorporating the early childhood education and child welfare perspectives that are important in understanding the impact that maltreatment has on the school readiness and academic achievement of young maltreated children. What follows next is a discussion of bioecological theory and how it provided a framework for this study.

\section{Bio-ecological Theory}

The use of a guiding conceptual framework provides a testable model with specifications of possible causal mechanisms for change (Flay et al., 2005). Bio-ecological theory framed this study by providing a lens to understand the multiple contexts and the proximal processes that influence development and academic achievement outcomes of children with a history of maltreatment. Bronfenbrenner and Morris (2006) described bio-ecological theory as a more "complex and more dynamic structure" (p. 794) that builds upon earlier forms of the model (i.e., social-ecological model), with a shift in focus from environment to a focus on processes within environments. The full model includes four interrelated properties (see Figure 4). The first is process, described as the core component of this model, which explains interactions between the individual, the environment, and a resulting outcome. More specifically, proximal processes that operate over time are proposed to be the primary mechanisms that lead to development. Proximal processes are thought to be most influential in early life (e.g., infancy and early childhood) and can be thought of as reciprocal interactions within the immediate environment of the person. To 
be most effective, or conversely more detrimental, these interactions must occur on a fairly regular basis and for an extended period of time (Bronfenbrenner \& Morris, 2006).

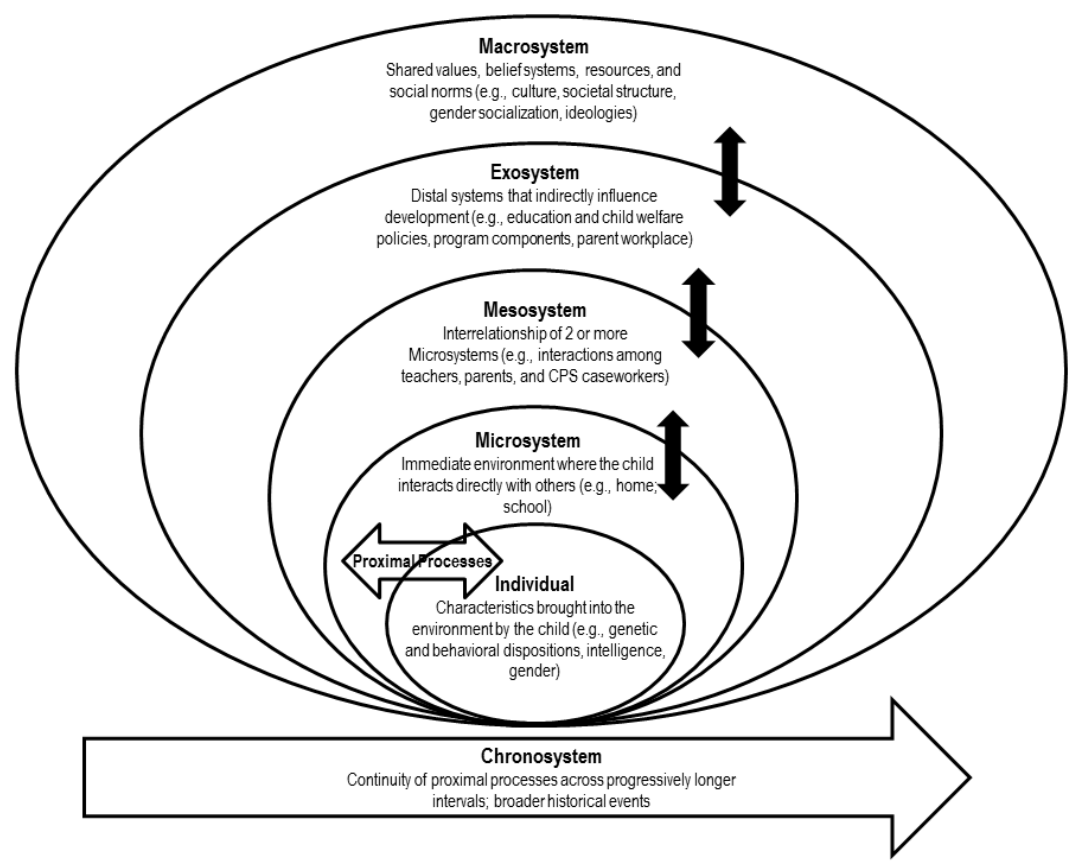

Figure 4. Bio-ecological model with interrelated systems that influence development over time. Adapted from Bronfenbrenner and Morris (2006) and Research and Training Center on Community Living (2013)

Importantly, proximal processes vary depending on the developmental outcome of interest, as well as the context within which development is occurring and the individual characteristics of the person. Additionally, timing in the life course is relevant in that complexities in proximal processes must mirror children's developmental capacities as they age, in order to remain effective in producing positive developmental outcomes. As children get older, persons with whom they interact regularly over time expand beyond parents to include other individuals such as teachers, peers, close friends, romantic partners, and coworkers. These proximal processes can be considered in the context of the development of school readiness skills. For example, Chazan-Cohen et al. (2009) found that the learning environment and 
maternal supportiveness were strongly associated with child vocabulary and letter-word knowledge, emotion regulation, and approaches to learning (key elements of school readiness) at age 5 .

The second property of the model involves the person. Though Bronfenbrenner noted the importance of genetic aspects of the individual, the second property of the model focuses on the characteristics that individuals bring to their environment (Tudge, Mokrova, Hatfield, \& Karnik, 2009; see Bronfenbrenner \& Ceci, 1994 for a detailed discussion on genetic influences). According to the model, there are three types of person characteristics that impact proximal processes and subsequent development: forces, resources, and demands. Force characteristics are thought to be behavioral dispositions that can set proximal processes into motion and sustain them (i.e., developmentally generative characteristics), or interfere with or prevent their occurrence (i.e., developmentally disruptive characteristics). Examples include differences in temperament, motivation, and persistence that impact development (Tudge et al., 2006). Resource characteristics represent abilities and liabilities of a person to engage effectively in proximal processes. Examples include chronic illness, intelligence, mental and emotional resources, as well as social and material resources (Bronfenbrenner \& Morris, 2006; Tudge et al., 2009). Finally, demand characteristics are those that invite or repel reactions from others that can disrupt or promote proximal processes. These may include gender, age, ethnicity, and physical appearance (Bronfenbrenner \& Morris, 2006; Tudge et al., 2009).

The third property, context, is the familiar component of Bronfenbrenner's ecological model and includes four interrelated systems: microsystem, mesosystem, exosystem, and macrosystem. The microsystem is defined as the immediate environment in which a person spends a majority of his or her time interacting with others. Examples of the microsystem include 
the home, school, workplace, and peer group. The mesosystem is a system of two or more microsystems and the relationship between them. The exosystem consists of important distal contexts or systems that indirectly influence development such as policies, programs, and the parent workplace. Finally, the macrosystem encompasses a group whose members have shared values and belief systems, resources, and social structures such as culture. Regarding school readiness factors, school and home fall under the context property. For children with a history of maltreatment and involvement with CPS, multiple layers of complex contextual factors influence their development.

The final property of the bio-ecological model is time. According to Bronfenbrenner and Morris (1998), there are three successive levels of time: microtime, mesotime, and macrotime. Microtime refers to the occurrence of proximal processes and its continuity or discontinuity. Mesotime is the collection of microtime across longer intervals such as days, weeks, and months that promote consistency of interactions. Macrotime refers to the broader historical events that may influence the individual's development. For example, developmental trajectories between children who were born before and after the introduction of CAPTA may differ due to the significant historical differences in how abuse and neglect were considered. Time and timing are considered to be important because other aspects of the model such as the process, person, and context are constantly changing over time (Tudge et al., 2009).

This Process-Person-Context-Time (PPCT) bio-ecological model provides a framework for examining the relation between maltreatment and children's academic functioning. Process factors, which are the engines of development, are central to understanding the development of children due to experiencing compromised caregiving early in life. The cognitive stimulation and emotional support that maltreated children receive from their parents, as well as foster parents 
can provide the support to improve emotion regulation and language skills. These supportive processes in turn, help promote school readiness and later academic functioning for children with a history of maltreatment.

Person characteristics are important to understand because certain characteristics of a person (e.g., temperament which may be associated with emotional dysregulation, language ability, learning disability) may serve as risk factors for ongoing maltreatment and poor academic outcomes. Alternatively, factors that contribute to resilience are important avenues to explore in order to promote positive outcomes. For example, a study by Zingraff, Leiter, and Johnsen (1994) found that positive school performance, as defined by grades, attendance, and decreased behavior problems in school, was associated with decreased delinquent behaviors.

Contextual factors are important in understanding how environments can increase or decrease the risk for poor academic outcomes. Limited access to positive environments such as improved parent-child relationships, high quality foster care, mental health services, or responsive schools could restrict exposure to the protective factors available for these children. For example, Font and Maguire-Jack (2013) found that for children aged 6 and older, foster care placement had a positive influence on school engagement, which may then serve as a protective factor for decreasing delinquency behaviors (Zingraff, Leiter, \& Johnsen, 1994). For young children, however, these context-level factors may be different. Preschool-aged children make up the second largest group of children investigated by Child Protective Services (CPS) and are more likely to be returned home (Stahmer et al., 2005). This means that young children involved with CPS may experience higher occurrences of instability due to movement between their family of origin home context and short-term foster care during the investigation period. Higher 
rates of instability in turn impact the quality of the caregiving environment that can promote or hinder socioemotional development of young children with a history of maltreatment.

Finally, it is important to understand how time can play a role in the academic development of preschoolers with a history of maltreatment. Examples include understanding policies (i.e., exosystem factors) in place at the time of involvement with the child welfare system that may then impact the proximal processes within these contexts. Additionally, disruptions in foster care placement or school attendance may serve as risk factors for lower academic achievement.

In sum, the PPCT model provides a framework for examining human development, not independent of context but in the bidirectional influence over time that shapes development. A clearer understanding of proximal processes allows for the design of targeted interventions given their importance in mediating the child's experience within multiple contexts (and the resulting outcomes). Proximal processes are critical to consider for preschool-aged children who experience maltreatment because proximal outcomes such as emotion regulation and language, or distal outcomes such as academic achievement, are a function of consistent and prolonged interaction with their immediate environment. Also essential to examine are the moderating effects provided by characteristics of the individual, the environment, and social changes as a result of growth, factors which are highlighted in subsequent sections of this review.

\section{School Readiness Skills in Early Childhood}

Building on the bio-ecological model, school readiness results from processes that occur between young children and their contexts. Children develop rapidly from birth to age 5 in the areas of language, cognition, emotion regulation, social skills, and moral capacities. Variability and disparities in knowledge and skills can have an impact on children's transition to formal 
schooling (NRC/IOM, 2000; Pianta, 2007). Differences in school readiness skills have been strongly associated with social and economic factors and are predictive of later academic performance (NRC/IOM, 2000). In addition, persistence of either positive readiness skills or problems in readiness domains is highly predictive of later attainment (Duncan \& Magnuson, 2011).

Given that early learning and development rest upon a wide range of experiences and individual characteristics, Kagan, Moore, and Bredekamp (1995) proposed 5 domains of school readiness that influence later academic performance. These are: (1) physical well-being and motor development, (2) social and emotional development, (3) approaches toward learning, (4) language development, and (5) cognition and general knowledge. The physical well-being domain is assessed using 3 categories. The first is physical development, which includes overall rate of growth, level of physical fitness, and body physiology. The second is physical abilities, which includes gross motor skills, fine motor skills, oral motor skills, sensorimotor skills, and functional performance. The final category addresses background contextual factors related to development such as perinatal context, caregiving environment, and health care use. Physical well-being was deemed important given that healthy children can focus on or actively engage in experiences that facilitate learning.

Emotional and social development, though under the same domain, are considered separate by Kagan et al. (1995). According to the authors, emotional characteristics involve the individual's feeling states about the self and others. Social characteristics pertain to the interaction of two or more individuals, especially with peers and adults, and refer to the interpersonal relationship and behaviors established with others. Social and emotional competence has often been associated with overall achievement and later life success. The 
authors added that these domains may be malleable and receptive to intervention for early childhood educators. More recent studies have incorporated emotion knowledge and emotion regulation under this socioemotional domain of school readiness (Blair 2002; McWayne, HahsVaughn, Cheung, \& Wright, 2012; Raver, 2002).

The approaches toward learning domain was defined as an umbrella term that covers a range of attitudes, habits, and learning styles of young children. It is assumed that approaches toward learning may be influenced by individual-level factors or predispositions such as gender, temperament, and cultural patterns. According to Kagan et al. (1995), learning proceeds more effectively when children employ their pre-existing abilities such as language skills or temperamental disposition to self-regulate. Simply acquiring knowledge, skills, and capacities was deemed insufficient for developmental success.

Language development is the acquisition of linguistic forms and procedures, as well as rules and norms for expression and interpretation (Kagan et al., 1995). The components of language are semantics (content or meaning), syntax (form or structure), and pragmatics (use or function). Language acquisition provides children with the means to communicate and share thoughts, ideas, or emotions with others around them. Because language includes the component of conventional norms and rules, this dimension is closely related to the socioemotional domain. The process of language development includes expressive and receptive language skills that require the cognitive component of language in order to access current knowledge and gain new knowledge from information heard in the environment.

The cognitive domain includes three kinds of knowledge: physical knowledge, logicomathematical knowledge, and social-conventional knowledge (Kagan et al., 1995). Physical knowledge is the knowledge of objects in the external reality (e.g., taste, texture, weight). 
Logico-mathematical knowledge establishes similarities, differences, and associations beyond the physical knowledge. Social-conventional knowledge includes agreed-upon conventions of society and culture. This includes school-learned knowledge that could not be reinvented by every cohort of students.

According to the National Research Council and Institute of Medicine (NRC/IOM; 2000), there is rapid development in early childhood of two specific domains of school readiness: socioemotional and language skills. Shonkoff and Phillips, the authors of this treatise, noted that during this time, children negotiate the transition from external to self-regulation. This includes learning how to regulate emotions, behavior, and attention. In addition, it is also during this time period that children learn to relate with others and form friendships. Finally, it is in the period of birth to 5 that children acquire the foundational skills that promote communication and learning, which includes early development of language, reasoning, and problem solving. Given the importance of the development of emotion regulation skills and language during early childhood, it is necessary to understand how these two sets of skills could be affected by early adversity, such as maltreatment. A more detailed discussion of emotion regulation and language are presented later in this chapter.

Research has documented that school readiness skills are important in the transition from preschool into kindergarten, and has identified specific clusters of school readiness skills and associated outcomes. For example, using a person-centered analysis, McWayne, Cheung, Wright, and Hahs-Vaughn (2012) identified 5 distinct patterns of school readiness for Head Start children during their first year of participation. Profile 1 was made up of average academic and social competencies, with low average behavior problems at home (28\%); profile 2 displayed high behavior problems at school, with low-low average social and academic skills (17\%); 
profile 3 showed high behavior problems at home (15\%); profile 4 displayed high social skills (12\%); and profile 5 encompassed students with high cognitive skills (19\%). The authors then examined how each of the profiles related to end-of-kindergarten outcomes. They found that children with more competent preschool profiles performed better over time and that these patterns at the start of the program were consistent predictors of later performance, controlling for demographic and contextual factors. Additionally, the authors found that both family and classroom factors further predicted later performance at kindergarten. These results highlight the importance of social and cognitive factors for later academic performance. Moreover, findings supported the importance of positive microsystems (i.e., classroom and family factors) and proximal processes (i.e., parent-child and teacher-child interactions) that further promote success. Finally, findings from this study supported the value of using person-centered methods for answering research questions.

Understanding the role of early adversity and its relation to school readiness can be undertaken with research on the transition to school for children with a history of maltreatment. Indeed, the normative transition to school is challenging even in the non-maltreated population; attainment of school readiness skills is important due to its impacts through elementary school and beyond (Duncan, Claessens, Huston, Pagani, Engel, Sexton, Dowsett, Magnuson, Klebanov, Feinstein, Brooks-Gunn, \& Duckworth , 2007; Rouse \& Fantuzzo, 2009). Thus, experiencing maltreatment and transitioning to school represents a difficult interactive experience that may place children at additional risk for poor academic outcomes. There has been very limited empirical attention devoted to the school readiness skills of children in the child welfare system. In one of a very few studies, risk for grade repetition peaked prior to third grade for both 
maltreated and non-maltreated children (Rowe \& Eckenrode, 1999). However, this difference was more severe for maltreated children.

Experiencing maltreatment during the early childhood period may impact school readiness through additional risk factors. These included increased developmental and behavioral needs, and decreased access to services (Stahmer, Leslie, Hurlburt, Barth, Webb, Landsverk, \& Zhang, 2005). Preschool-aged children were also more likely to remain in their families of origin, with less access and use of services, which would then place them at increased developmental risk upon entry into child welfare (Stahmer, et al., 2005). In a similar study conducted by Zimmer and Panko (2006) using the NSCAW dataset, they found that preschoolaged children displayed more developmental delays than school-aged children and that only $38 \%$ of those with delays received available services.

In sum, school readiness represents a confluence of skills that reaches across developmental domains. Language and emotion regulation are rapidly developing skills during early childhood that promote school readiness. The school readiness skills of preschool-aged children entering the child welfare system have been understudied. Extant data suggest that preschool-aged children experience a different set of challenges over their non-maltreated counterparts as they enter the child welfare system. This, in turn, may adversely affect their school readiness skills and later academic achievement. These children appear to show increased needs and lower access or use of services, which immediately places them at risk upon entry (Stahmer et al., 2005). In addition, they may have experienced prolonged exposure to adversity compared to children who were removed during infancy or toddlerhood.

Moreover, these children are entering the child welfare system at a sensitive time for the development of language and emotion regulation skills. Taken together, these findings, along 
with those documenting the difficulty of transitioning into school and the adverse outcomes emanating from prior experiences of maltreatment, suggest that these children are at risk for negative effects on their development, particularly in the school readiness domains of emotion regulation and language. Thus, it is essential to fill the large research gap relevant to the school readiness of maltreated children in the child welfare system.

\section{Academic Achievement: Elementary to Adolescence}

Unmitigated risk factors experienced during early childhood could affect children's school performance beyond preschool. Thus, it is important to consider the academic outcomes of children with a history of maltreatment due to the potential pernicious consequences of school failure for this population of children (Vacca, 2008). This section reviews studies that have examined academic outcomes associated with maltreatment along with related risk factors that exacerbate this relationship.

\section{Attendance}

Physical presence in school is important for learning and engagement to occur. Unfortunately for children who experience maltreatment and involvement with the child welfare system, attendance rates severely decline immediately after the initial Child Protective Services (CPS) report. Absenteeism rates remain stable regardless of case substantiation (Leiter, 2007; Leiter \& Johnsen, 1997; Rouse \& Fantuzzo, 2009); that is, declining rates of attendance continue despite official CPS findings as to whether maltreatment has occurred. Thus, the increase in absenteeism may be attributed to the placement and school disruptions often experienced by children when they first come into contact with CPS. In one study, these findings were robust even after controlling for known covariates of attendance problems such as poverty (Perlman \& Fantuzzo, 2010). 
A study by Zorc, O’Reilly, Matone, Long, Watts, and Rubin (2013) examined the relation of attendance to foster care placement and reunification with biological parents. They found a stepwise increase in absenteeism as young, school-aged children's foster placements were disrupted. Additionally, children who reunified with their biological parents showed the highest rates of absenteeism when compared to those in both stable and unstable placements. These results underscore the risk that child welfare involvement places on maltreated children through decreased stability in their school microsystem, as well as the peer and family microsystems. When a stable and consistent school context is unavailable, regular interaction between teachers, family, and children cannot occur. These regular interactions between the school and family microsystems are needed in order to promote trust and support between the home and school environments that support children's positive school outcomes. Without these prolonged interactions, it becomes difficult for development-promoting proximal processes to occur and to increase positive outcomes related to Grade Point Average (GPA), special education placement, and standardized test performance.

\section{Grade Point Average}

Studies have documented an association between maltreatment and decreased GPA, a measure of academic achievement appropriate for older children (Kendall-Tackett \& Eckenrode, 1996; Slade \& Wissow, 2007). One study examined sibling pairs of U.S. middle and high school students (Slade \& Wissow, 2007) and found that prior to $6^{\text {th }}$ grade, severity of childhood maltreatment was significantly associated with lower GPA, as well as problems completing homework. However, the authors found that children who scored higher on the Peabody Picture Vocabulary Test (PPVT) language measure were less likely to have lower GPA, indicating a 
potential protective factor of language skill. The effects on lower GPA appeared to be present regardless of maltreatment type (Kendall-Tackett \& Eckenrode, 1996).

It is interesting to note that Leiter (2007) found the rate of decline for GPA did not follow the same trajectory as absenteeism noted earlier. Unlike absenteeism, which sharply increases after initial CPS involvement, the decline in GPA occurred later in the process. The author attributed this difference in rate of decline to a potential protective response by CPS by removing children from their adverse family of origin and placing them into foster care.

\section{Special Education Placement}

Early experiences of maltreatment have been found to contribute to the risk of special education placement. One study documented that $1 / 5$ or $20 \%$ of infants and toddlers who were investigated by CPS had an Individualized Education Plan (IEP) by the second grade (Scarborough \& McCare, 2010). This figure, however, may underestimate the actual prevalence of children who are in need of special education services. According to a report by Casanueva, Wilson, Smith, Dolan, Ringeisen, and Horne (2012), 54.5\% of the children in the second National Survey of Child and Adolescent Well-being (NSCAW II) had a Bayley Infant Neurodevelopmental Screener (BINS) score that was indicative of high risk for developmental delay or neurological impairment at baseline (i.e., infants and toddlers at the start of the study), suggesting that more than half of the very young children in child welfare may benefit from special education services.

Additionally, for children who were placed in foster care, it appears that there is a disproportionately large representation in special education placements (Stone, 2007). According to Stone (2007), foster children were placed in special education categories that included emotional disturbances and learning disabilities. The author reviewed evidence on the 
appropriateness of special education placement and found that foster children performed similarly in their special education classes to their non-foster counterparts. However, she also identified unique issues for children in foster care, such as difficulties with interagency collaboration, educational advocacy needs, and student mobility.

Differences in regulatory and programmatic processes may exist between the education and child welfare systems that make interagency collaboration difficult. For example, child welfare policies that ensure child and family confidentiality may make it difficult to have information shared for an Individualized Education Plan (IEP) process. Further, advocacy requiring parent/guardian presence may be different for foster children. This may be due to parent consent often being required during the IEP process. Unfortunately for children in foster care, guardianship may be unclear and consent may not be provided in time. When parent or guardian involvement is delayed, then educational advocacy cannot proceed. Finally, due to the high instability in home placement, school mobility may occur more frequently. For these students, high mobility may delay or halt the assessment processes for access to services, as well as contribute to discontinuity of intervention services.

\section{Standardized Test Scores}

Crozier and Barth (2005) examined cognitive and academic functioning of school-aged participants $(\mathrm{N}=2,368)$ from the National Survey of Child and Adolescent Well-being (NSCAW) study. They found that on average, maltreated children scored significantly below national norms on the Woodcock-McGrew-Werder Mini-Battery of Achievement in reading (MBA Reading) and mathematics (MBA Math). The authors also found that risk factors acted cumulatively to predict decreased performance. These findings were replicated in a study by Rouse and Fantuzzo (2009) in which cumulative risk factors predicted lower academic achievement outcomes 
measured by reading achievement, mathematics achievement, and promotion to third grade. The authors recruited participants from a large city in the Northeast. There were 10,349 children enrolled in second grade who were included in the final sample. These authors found that maltreatment provided the largest odds ratio in predicting lower academic outcomes for a representative sample of low-income, school aged children in an urban public school system.

Coohey, Renner, Hua, Zhang, and Whitney (2011) conducted a study using NSCAW data to examine characteristics of the maltreatment experience (i.e., maltreatment type), child characteristics, and contextual characteristics to examine academic achievement over time for a nationally-representative sample of maltreated children. The final sample consisted of 702 children between the ages of 6 and 10 years old at the time of the report. The authors examined academic achievement from waves 1, 2, and 3 using the math and reading scores from the MBA reading and MBA math subtests of the Woodcock-McGrew-Werder Mini-Battery of Achievement. The authors used Barnett et al.’s (1993) Maltreatment Classification System (MCS) to classify maltreatment experience as physical abuse only, physical neglect only, supervisory neglect only, and other types of maltreatment. Additional measures included caregiver substance abuse, mental health problems, daily living skills, intelligence, behavior problems, relationship with peers at school, and school engagement.

Results from the study indicated that chronic maltreatment, poor daily living skills, and lower intelligence explained substantial variance in lowered math scores for maltreated children, whereas maltreatment type, poor daily living skills, and lower intelligence explained differences in lowered reading scores. However, the authors reported that the effect for children's behavior was not in the predicted direction. That is, chronically maltreated children with behavior problems showed better math scores than those without behavior problems. One potential source 
of problem for this finding is the inappropriate application of the MCS in delineating maltreatment type and chronicity. Use of the MCS requires a thorough review of case records, which was not available in the NSCAW data. Additionally, variations in these scores may be due to other factors not considered in the model (e.g., emotion regulation and language).

Taken together, these findings point to the educational vulnerabilities that maltreated children experience once they have entered school. Examining the school readiness of maltreated children involved with CPS can facilitate an understanding of the intra-individual and contextual factors which may lead to their long-term academic challenges. Specifically, it is essential to examine the role that emotion regulation and dysregulation play in early childhood to foster school readiness and later academic achievement.

\section{Bio-ecological Factors and Maltreated Children}

There are many bio-ecological factors specific to children with a history of maltreatment that are relevant to an understanding of their school readiness and academic outcomes. The bioecological framework proposes a mechanism for examining the development of these children within a person-process-context-time perspective. According to the bio-ecological model, the regular interactions (known as proximal processes) between biological/individual factors and the ecology in which the child develops are important in promoting positive academic outcomes.

Key components of the model in relation to the biological/individual (i.e., emotion regulation, language, and gender), contextual (i.e., school, home placement), time (i.e., duration of placement), and proximal processes (i.e., caregiver-child interactions) specific to children with a history of maltreatment will be discussed. 


\section{Definition of Emotion Regulation}

Emotion regulation is an unobserved phenomenon, which requires a detailed definition of the construct. Prior to reviewing the literature on how this core developmental process is affected by maltreatment, it is important to offer a synthesized definition and model to understand what is being studied. To begin, three definitions were incorporated for this study in order to understand what emotion regulation is. A brief discussion of the process model of emotion regulation will be discussed in order to understand what behavioral indicators might represent this construct.

This study incorporated three definitions of emotion regulation that build upon each other. First, emotion regulation is a modulation of emotion and behavior that helps facilitate interaction between the individual and the environment (Thompson, 1994). Looking at emotion regulation from this perspective allows us to explore the environment and context (e.g., school, peer, family) within which emotion regulation may occur.

Second, emotion regulation refers to changes in emotion (e.g., duration and intensity) or other psychological processes (e.g., social interaction or memory) due to activated emotions (Cole, Martin, \& Dennis, 2004). Thus, emotion regulation is defined as a construct that resides in both the emotional and cognitive domains simultaneously. Additionally, it defines the added complexity of emotions by looking at valence, intensity, and duration.

Finally, emotion regulation can be construed as a system composed of cognitive, behavioral, and emotional aspects of functioning that enables effortful control of attention, behavior, and emotion for the purpose of goal-directed behavior (Blair \& Raver, 2012) or adaptive functioning (Calkins, 1997). This definition incorporates the dynamic nature of cognitive, behavioral, and emotional components of emotion regulation, as well as its importance in the way an individual interacts with his or her environment to accomplish a particular goal or 
adapt to particular situations. Effortful control within this definition reflects the reactive and active role of the individual in the process of self-regulation and self-control.

The process model of emotion regulation provides a framework for understanding selected emotion regulation strategies that occur before, during, or after an emotion-generative process (Gross \& Thompson, 2007). Emotion regulatory or dysregulatory processes can be assumed to be a component of the person or individual factor of the bio-ecological model. Thus, integration of the process model within a bio-ecological framework provides a specific mechanism that explains the construct of emotion regulation or dysregulation. There are five emotion regulation processes on a temporal spectrum that indicates the point of deployment (see top part of Figure 5). These are placed in two broad categories, antecedent-focused (processes that occur before emotion generation) and response-focused (processes that occur after emotion generation).

The antecedent-focused strategies include situation selection, situation modification, attentional deployment, and cognitive change. Situation selection involves taking actions that make it more or less likely that a desirable or undesirable emotion will be generated based on the situation that a person chooses to be in. Situation modification involves actively modifying external, physical environments in order to alter its emotional impact. Attentional deployment refers to the way in which individuals direct their attention within a given situation in order to influence their emotions. Finally, cognitive change involves shifting appraisals of the situation in order to alter its emotional significance. This is done by changing one's thoughts about the situation or changing one's capacity to manage the demands it poses. Response-focused processes include response modulation, which occurs later in the emotion generative process, and refers to physiological, experiential, or behavioral responding as directly as possible. 


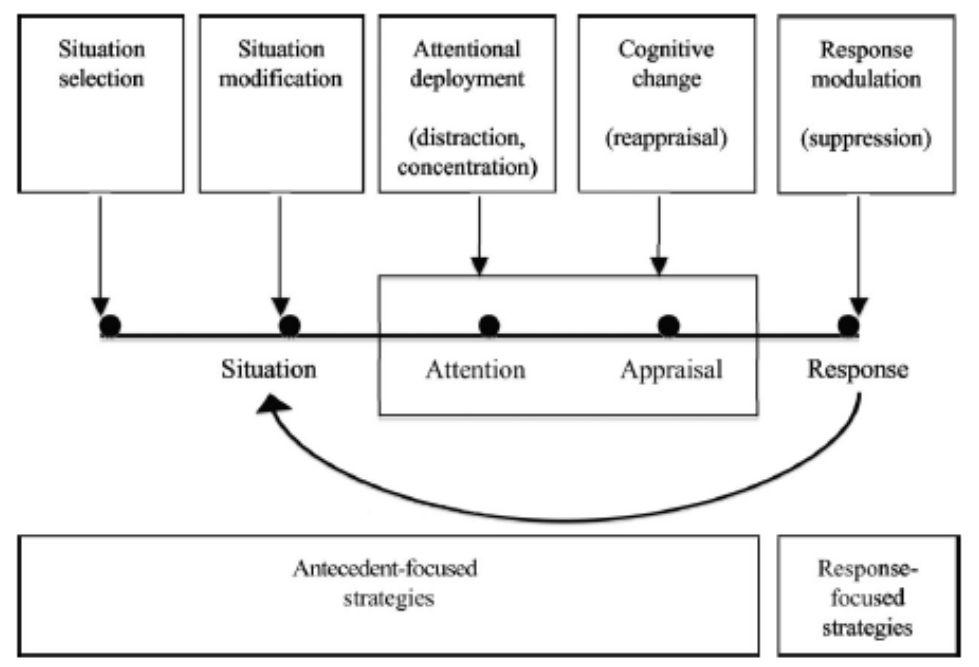

Figure 5. Process model of emotion regulation Source: Gross and Thompson, 2007; Webb, Miles, and Sheeran, 2012.

A meta-analysis was conducted by Webb, Miles, and Sheeran (2012) in order to evaluate the effectiveness of each of the emotion regulation strategies described above. The authors conducted a systematic search of the literature and identified 306 experimental comparisons of different emotion regulation strategies and categorized the outcomes based on the model above. They found that attentional deployment had no effect on emotional outcomes $(d=0.00)$, cognitive change had a small to medium effect $(d=0.36)$, and response modulation had a small effect $(d=0.16)$. These results are important to keep in mind (e.g., the essential nature of the cognitive change/shifting appraisal aspect of emotion regulation), particularly in understanding the relationship between emotion regulation and academic achievement measures.

Regulatory strategies that children select when faced with an environmental stimulus (e.g., high demands of school) may predispose them to certain response biases (i.e., responsefocused). These response biases may be associated with attentional bias (i.e., antecedentfocused) and effortful control (response-focused) of reactive prepotent responses (i.e., biological factors such as temperament). Therefore, behavioral problems such as externalizing or 
internalizing problems can be alleviated through appropriate development of emotion regulation strategies when children are able to inhibit their predominant response, maintain attention, and accurately assess emotional cues. This in turn would support successful readiness for school and subsequent academic outcomes. However, because of the experience of early maltreatment, children may exhibit compromised emotion regulation skills leading to behavior problems, psychopathology, attentional bias, peer relationship problems, and emotion identification problems, which compromise school readiness (Brown, Ackerman, \& Moore, 2013; Kim \& Cicchetti, 2010; Maughan \& Cicchetti, 2002; Romens \& Pollak, 2012; Teisl \& Cicchetti, 2008).

\section{Emotion Regulation and Maltreatment}

Emotion regulation can be construed as a mechanism that mediates the relationship between maltreatment and academic achievement measures (Graziano, Reavis, Keane, \& Calkins, 2007). Although the research in this area is limited, there are a few studies that examine the linkage between maltreatment and emotion regulation. Manly, Kim, Rogosch, and Cicchetti (2001) conducted a study that compared 492 maltreated and 322 non-maltreated children who participated in a summer day camp to examine their internalizing and externalizing symptomatology. According to the authors, they found that physical abuse during the preschool period predicted externalizing behaviors, whereas physical neglect occurring in the same time period significantly predicted internalizing symptoms. They added that the presence of chronic maltreatment, particularly if its onset occurred during infancy through the preschool period, predicted an increase in maladaptive outcomes by school age.

Building on the work of Manly et al. (2001), Pears, Kim, and Fisher (2008) examined how subtypes of maltreatment co-occurred and how experiencing multiple types of maltreatment was associated with cognitive functioning and behavior problems. The authors reviewed case 
records and employed the Maltreatment Classification System to code for the following maltreatment types: physical abuse, sexual abuse, physical neglect (i.e., parental failure to provide adequate food, clothing, shelter, medical care, or a safe living environment for the child), supervisory neglect (i.e., parental failure to provide age-appropriate supervision for the child), emotional maltreatment (i.e., parental rejection, abandonment, or allowing the child to be witness to traumatic events), educational maltreatment (i.e., parental failure to send the child to school), and moral/legal maltreatment (i.e., parents using the child for illegal purposes).

Using Latent Profile Analysis on 117 maltreated foster children between the ages of 3 and 6 , the authors came up with four distinct profiles that showed moderate to high levels of maltreatment. Profile (a) included supervisory neglect and emotional maltreatment; (b) included sexual abuse, emotional maltreatment, and neglect; (c) included physical abuse, emotional maltreatment, and neglect; and (d) included sexual abuse, physical abuse, emotional maltreatment, and neglect. Profiles (b), (c), and (d) used the broad category "neglect" because the occurrence levels for physical and supervisory neglect were similar. In creating these latent profiles, the authors were able to look at the interactive process of maltreatment types that these children often experience. In a secondary analysis of profile membership and its relation to cognitive functioning, externalizing, and internalizing symptoms, the authors found the following: profiles that included neglect, physical abuse, or both, but excluding sexual abuse showed lower cognitive functioning overall; externalizing problems were highest for children who were in profile (d); and internalizing problems were highest for children who belonged to profiles that included physical abuse, sexual abuse, or both.

In both of these studies, timing of maltreatment may have influenced how children organized their emotion regulation strategies in response to their compromised caregiving (i.e., 
proximal process). That is, maltreatment occurring between infancy through the preschool period could be associated with children's capacity to organize response-focused regulation strategies around internalizing and externalizing behavioral patterns. Unfortunately, the presence of maladaptive outcomes (e.g., behavior problems, emotion regulation, and attentional biases) as a developmental consequence of maltreatment has long-lasting effects on academic success, peer relations, and persistent or worsening behavior problems (Maughan \& Cicchetti, 2002; Woodruff $\&$ Lee, 2011).

Maughan and Cicchetti (2002) examined the effect of maltreatment and interadult violence on children's emotion regulation abilities and socioemotional adjustment. They looked at emotion regulation patterns for 139 4- to 6-year olds (88 maltreated and 51 non-maltreated). The authors found that for children who experienced maltreatment along with witnessing interadult violence, a high prevalence of emotion dysregulation in the form of over- or underregulation was indicated. Moreover, it appeared that regulatory difficulties was a result of the children's increased attention to the negative stimuli. They experience difficulty transitioning away from the dysregulated response despite the termination of the negative stimuli, possibly indicating difficulties with antecedent-focused regulation strategies (i.e., attentional bias). The authors' findings are consistent with the literature that shows attentional bias towards negative stimuli experienced by children with a history of maltreatment. Oftentimes, these attentional problems could be perceived as a symptom akin to Attention Deficit Hyperactivity Disorder (ADHD) due to the child's inability to focus, or use of distraction as a regulatory strategy to address negative emotions. Additionally, attentional biases to emotionally salient cues may also lead to other externalizing or internalizing problems as children's response biases may be influenced by paying more attention to negative contextual cues. 
Pears, Fisher, Bruce, Kim, and Yoerger (2010) examined the role of inhibitory control and caregiver involvement in mediating the associations between maltreatment and foster placement, as well as early school adjustment. The authors recruited 117 maltreated foster children and their foster parents, and a community comparison group of 60 age- and SESmatched, non-maltreated children living with their families of origin. The children were between 3-6 years old and participated in the study for a period of 24 months. School adjustment was defined as academic competence, which was measured using a composite measure of teacher report and school records, and social-emotional competence, which was measured using a composite score comprised of three domain scores: prosocial behavior, emotion regulation, and behavior regulation. Mediators included inhibitory control and caregivers' involvement in schooling. Results indicated that inhibitory control fully mediated the effects of maltreatment and foster placement on academic and social-emotional competence. Additionally, the authors found a significant indirect path from maltreatment and foster care placement to social-emotional competence through caregiver involvement.

Emotion understanding is a cognitive/appraisal process that is a component of emotion regulation. Multiple studies have examined this construct in maltreated children and documented a deficit in emotion understanding for maltreated children compared to their non-maltreated counterparts, and a potential hypersensitivity to specific emotions (Luke \& Banerjee, 2013). However, there are only two studies of emotion knowledge in maltreated children in foster care. In a study by Pears and Fisher (2005), the authors investigated a sample of 3- to 5-year old maltreated foster children and a comparison non-maltreated sample. The authors concluded that the maltreated sample displayed significantly worse emotion understanding and theory of mind capabilities (using emotion understanding tasks and theory of mind tasks). According to the 
authors, these findings provide clear evidence of cognitive and emotional deficits in children living in foster care who had prior experience of maltreatment. Further, Jones Harden, Morrison and Clyman (2014) investigated emotion understanding in a group of children transitioning to school (ages 4-6) who were maltreated and placed in foster care. These authors found that children's receptive vocabulary was a major contributor to their emotion understanding, underscoring the importance of language as a protective factor to social-emotional competence in this population.

From a process model perspective, the studies described above indicated that children who experience maltreatment early in life (i.e., infancy through preschool) show deficits in regulatory strategies when managing emotions. These include antecedent-focused strategies such as attention (e.g., problems disengaging attention from negative salient cues and attentional bias towards negative salient cues) and appraisal (e.g., emotion understanding), as well as responsefocused strategies such as behavior problems (e.g., externalizing and internalizing behavioral response patterns). Compromised regulatory strategies could make it difficult for children to effectively attain goals and adapt appropriately, particularly when related to the home and school context (Haskett, Stelter, Proffit, \& Nice, 2012). Additionally, communicating about elicited emotions and difficulties could provide maltreated children with an alternate response-focused strategy to help regulate emotion. However, children with a history of maltreatment exhibit emotion understanding and language delays that may make awareness and communication of emotions challenging (Eigsti \& Cicchetti, 2004, Jones Harden et al., 2014).

\section{Language Development and Maltreatment}

Evidence on the relationship between language and school readiness was presented by Cristofaro and Tamis-LaMonda (2011) in a longitudinal examination of the relationship between 
75 low-income mothers and their children, and how the quality of this relationship contributed to children's oral language. Lexical diversity was assessed using the Peabody Picture Vocabulary Test (PPVT-III) while mothers' use of wh-questions (e.g., who, what, where, why, when, or how) were assessed using video recorded play interactions. At pre-kindergarten, mother-child dyad narratives were coded and children's school readiness was assessed using a composite based on print word knowledge, letter-word identification, math skills, and sustained attention. The authors found that quality and diversity of mother-child conversations predicted children's PPVT scores, which in turn predicted school readiness. These results highlight the protective potential of the positive interaction (i.e., proximal processes) between parent and child in promoting language development. However, for children with a history of maltreatment and child welfare involvement, this protective factor may not be available. It is therefore important to understand how maltreatment affects language development.

There is a paucity of evidence regarding maltreated children's language skills, particularly for those children who have entered the child welfare system. In a report prepared by the Research Triangle Institute (2008) using NSCAW data, it was documented that $30 \%$ of children between the ages of zero and three fell behind non-maltreated children in both cognitive and language skills. For those children who were 2 years or younger at wave 1, a follow-up assessment 18 months later revealed that cognitive and language measures continued to fall below average. Overall scores on the Kaufman Brief Intelligence Test (KBIT) indicated that maltreated children fell half a standard deviation below the mean for children in general. Scores on the Preschool Language Scales (PLS) were in the average range, though significantly lower than non-maltreated children. Total and expressive language scores were half a standard 
deviation below the mean. These results provide evidence that maltreated children exhibit compromised language skills.

To further understand what structure of language might be specifically affected, Eigsti and Cicchetti (2004) examined the impact of maltreatment on the expressive syntax of 19 maltreated and 14 non-maltreated preschool-aged children. The authors observed mother-child dyads in play sessions. For the maltreated group, the study recruited biological mothers who had been named as the perpetrator or co-perpetrator in the opened CPS cases. The language outcomes were assessed using the Index of Productive Syntax as a means of evaluating differences in syntactic development, and the Peabody Picture Vocabulary Test-Revised (PPVTR) to measure receptive language development. Results supported the authors' prediction that maltreated children would exhibit syntactic delays at age 5, which means producing less language and less advanced vocabulary. Results also indicated that maltreating mothers had fewer utterances directed towards their children. Additionally, the maltreated group displayed vocabulary skills in the low average range compared with the average range of the comparison group. The authors suggested that this could indicate that maltreatment may lead to failure of development across multiple areas of language development.

A study by Pears, Heywood, Kim, and Fisher (2011) examined the reading skills of children in foster care and its relationship with academic outcomes at kindergarten. They recruited 63 children in foster care $(M$ age $=5.46$ years, $S D=0.36)$. Fifty-nine percent were in nonrelative care, with an average of 3 unique foster placements $(S D=1)$ and an average of 558 days in care $(S D=397)$. Language was assessed through phonological and phonemic awareness, alphabet understanding, and oral language ability. A multivariate path analysis was conducted to examine the authors' questions. Results indicated that the final selected model showed that only 
phonological awareness was a unique significant predictor of teacher-rated early literacy skills. The other pre-reading skills covaried with each other and accounted for a significant amount of variance in teacher ratings. Due to this covariation, it would be difficult to disentangle the effects uniquely provided by the other skills. The authors also reported that on the measure of phonological awareness, $54 \%$ of foster children in the study scored below the $23^{\text {rd }}$ percentile, placing them at risk for learning problems.

In a review by Stock and Fisher (2006), the authors examined policy and practice implications related to language delays among foster children. According to the authors, language is a central process in the development of young children's cognitive and socioemotional skills. During the sensitive period of development, language may be susceptible to negative environmental influences such as maltreatment and foster placement. Unfortunately, the prevalence rates of language delays for foster children are very high and these problems persist throughout the school years. Negative developmental effects can be seen in literacy, social competence, and school readiness. To address the negative link between maltreatment and language, the authors recommended that policy and interventions designed to help promote positive language development must be addressed at an early age. It is during the sensitive period from birth to preschool that relative plasticity exists and that this is an optimal time for intervention efforts. According to the authors, however, research on foster children indicates that screening and evaluations are often underused and imprecise that these delays go unnoticed.

There is a preponderance of evidence that language skill is a critical component of school readiness. Further, the quality of the caregiver-child interaction has been implicated in the development of language. Although the research is limited, it does appear that children with a history of maltreatment exhibit problems with expressive and receptive language. These 
language delays have also been found among maltreated children in foster care (Stock \& Fisher, 2006) and have long-term consequences for school readiness and later academic achievement.

\section{Emotion Regulation and Language}

According to Kagan et al. (1995), when studying school readiness, it is important to consider the interrelatedness of its dimensions. For example, temperament as a defining variable can be seen in more than one dimension of school readiness. These dimensions must then be considered as a whole, with no single dimension acting as a substitute for the complexities of early learning and development. In fact, the authors asserted that the concept of readiness must not be established as a unidimensional construct and cautioned against developing a single cutoff point for school readiness. Rather, child readiness must be considered as episodic, variable across the domains, and contextually influenced. For these reasons, emotion regulation and language will be examined concurrently in this proposed study.

Raver, Garner, and Smith-Donald (2007) described the interrelatedness of emotion regulation and language in the context of emotion knowledge and discussed its implication in promoting or obstructing learning in young children. The authors highlighted that young children develop competence in their ability to identify, label, and communicate emotions in addition to learning how to manage their emotions. The development of internal state language to describe feelings, concerns, and desires of self and others develop around the age 2 . It is at this point that internal state language plays a self-regulatory function in children that helps them label their emotion and employ regulatory strategies to manage their expression. From this perspective, receptive language skills appear to play a role in regulating emotions. Specifically, the ability for children to understand his or her emotions and those of others appear to play a role in peer and teacher relationships. This, in turn, facilitates a negative or positive learning environment. 
Monopoli and Kingston (2012), in their study of 67 second-grade students from a university town in rural Pennsylvania, examined how emotion regulation and language predicted social competence, and then examined how language mediated the relationship between emotion regulation and social competence. The authors found support for their hypothesis that emotion regulation predicted social competence. However, this relationship was not significant for language. Additionally, when language was included as an indirect path between emotion regulation and social competence, the authors did not find any significant effects for full or partial mediation. Based on the model presented by the authors, the null findings may be related to the proposed directional effect of emotion regulation to language. Given the proposition by Raver, Garner, and Smith-Donald (2007), temporal order between language and emotion regulation may have been reversed. According to these authors, language serves a regulatory function in young children, which assumes that language should precede emotion regulation.

This temporal order was echoed by Cole, Armstrong, and Pemberton (2010) in examining the role of language in the development of emotion regulation. Specifically, the authors discussed four diverse lines of research that examined how expressive language contributes to the development of self-regulation. Expressive language provided a more articulate means than emotion expression for communicating goals, a tool to enhance understanding of self and others' emotions, and an additional strategy for guiding and regulating emotions and actions. The authors noted that evidence still lacks in understanding how language specifically contributes to emotional expressivity.

A study by Moreno and Robinson (2005) specifically examined this association between language and emotional expressivity across infancy and toddlerhood. In their study, however, the authors adopted a similar temporal order to that of Monopoli and Kingston (2012) where 
emotion regulation preceded language. The authors recruited 77 children and their families who participated in Early Head Start programs. Observational methods were used to code joy and anger emotion elicitation. Using an additive regression model, the authors used joy and fear to predict 24- and 36-month scores on language measures. Results indicated that emotional vitality of positive and negative valence emotions (i.e., emotional expressivity) in 8-month olds accounted for significant variance in expressive language at 30 months.

Based on the evidence presented above, it is important to examine emotion regulation and language as interrelated components of school readiness. Indeed, the ability to verbally communicate one's goals and emotions can mitigate externalizing behaviors. Additionally, the ability to understand others' emotions helps facilitate social competence. However, contradictory evidence on directionality of the influence between language and emotion regulation warrants further attention. In addition, evidence of the interrelatedness of these readiness domains in maltreated children is virtually non-existent.

\section{Gender}

There is evidence of gender differences in behavior problems, language delays, and special education placements, wherein boys are likely to have increased probability of occurrence (Scarborough \& McCrae, 2010). For example, Denham, Bassett, Thayer, Mincic, Sirotkin, and Zinsser (2012) employed an observational assessment tool to examine the extent to which emotion regulation, gender, and relationship skills are related to academic success for preschoolers in a Head Start program. The authors found that boys, compared with girls, exhibited higher aggressive and pleasure-seeking behaviors. They added that teacher-reported negative school adjustment was more likely for boys with negative emotions or aggressive behaviors. A study by Keenan and Shaw (1997) specifically looked at gender differences in 
problem behaviors during the preschool period. One of the main findings of this study showed that girls displayed internalizing disorders (i.e., depressed mood and anxiety), whereas boys showed more externalizing disorders (i.e., conduct, defiant or oppositional behaviors, problems with attention, hyperactivity, and impulsivity).

Whitney, Renner, and Herrenkohl (2010) explicitly examined the relationship of gender with risk and protective factors affecting academic outcomes. The authors employed Latent Profile Analysis (LPA) to explore the possible heterogeneity of risk/protective variables. Classes were then used to predict academic performance. The initial sample $(N=457)$ recruited included $248(54 \%)$ boys and 209 girls (6 to 11-years old) from 297 low-income families. Families included child welfare involvement with both substantiated and unsubstantiated cases, as well as Head Start and community families. The authors found that the high risk, low protection class of children was more likely to experience low academic performance regardless of gender. For females, parental/peer disapproval of antisocial behavior served as a protective factor. However, this finding was not present for boys.

Based on the evidence presented, it appears that academic outcomes, emotion regulation strategies, and protective factors differ by gender. Boys appear to employ more externalizing regulation strategies, whereas girls appear to employ more internalizing strategies. These differences based on gender are important to consider in the context of maltreatment. For example, regulation strategy selection such as aggressive or externalizing behaviors may be more amplified with the experiences of early adversity for boys. This is particularly important if the child experiences severe maltreatment that is associated with more negative behaviors (Pears, Kim, \& Fisher, 2008). Additionally, because the child develops in context, aggressive behaviors may elicit negative perceptions from teachers, which may negatively impact proximal processes 
between student and teacher, and subsequently increase the risk and severity of academic problems already present for maltreated children.

\section{School Context}

Evidence for the importance of the preschool context for promoting school readiness has been documented (Maier, Vitiello, \& Greenfield, 2012; Mersky, Topitzes, \& Reynolds, 2011; Zhai et al., 2010). The study by Maier, Vitiello, and Greenfield (2012) examined the relationship between teacher-reported child-level social strengths, observed classroom process quality, and language and literacy growth. Using a sample of 275 preschoolers from 29 Head Start classrooms, the authors found that child-level factors predicted initial levels of language and literacy, while classroom organization predicted growth in language skills. These findings highlight the importance of understanding how child-level factors and school context interact to promote positive proximal processes to effect change in children's development.

However, in considering the multiple contexts in which children in the child welfare system are involved, a stable and consistent school context may not be available. Foster children in particular are at high risk for increased school mobility (Conger \& Finkelstein, 2003; Conger \& Rebeck, 2001). This relationship is important to consider given that school mobility has been associated with poor academic outcomes (Kerbow, 1996; Rumberger \& Larson, 1998;

Rumberger, 2003). Conflicting evidence exists, however, in the nature of the association between foster care placement, school mobility, and academic outcomes. For example, Font and MaguireJack (2013) examined a sample of children from the National Survey of Child and Adolescent Well-being (NSCAW) dataset and used propensity score matching based on demographic characteristics, maltreatment information, and prior school engagement. The authors found that children who were later placed in foster care displayed higher levels of school engagement 
(defined as cognitive and emotional engagement) when compared to maltreated children who remained home. Based on these conflicting results, the quality of the school and home environment may need to be further examined to explicate potential mediating factors influencing school performance.

\section{Out-of-Home Placement Context}

It is important to take into account the quality and stability of foster homes to examine associations with developmental outcomes for foster children (Font \& Maguire-Jack, 2013; Stacks \& Partridge, 2011). As mentioned in the previous paragraph, placement stability impacts children's school placement and academic performance. Increasing the number of placements (i.e., increasing instability) and duration of foster care (non-permanent) placement, as well as the mobility between home and out-of-home care, have been associated with negative outcomes for children (Jones Harden, 2004; Lloyd \& Barth, 2011; Ryan \& Testa, 2005; Stacks \& Partridge, 2011). According to the bio-ecological model, it could be hypothesized that negative outcomes may potentially be related to the lack of stability and consistency with respect to the interactions between the child and his or her environment (i.e., proximal processes). That is, without the presence of a regular and consistent caregiver, it would be difficult for maltreated children's core developmental processes of language and emotion regulation skills to develop, skills that are critical for school readiness. In order to mitigate the negative developmental outcomes for children with a history of maltreatment, stable, consistent, and responsive caregivers are necessary (Alink, Cicchetti, Kim, \& Rogosch, 2009; Jones Harden \& Whittaker, 2011; Son \& Morrison, 2010). 


\section{Family of Origin Home Context}

There is scant evidence examining the direct role of the family of origin (i.e., biological parents) as a context for preparing or diverting maltreated children's school readiness. However, the experience of maltreatment occurring within the home may be examined as a proxy for proximal processes within this context (Son \& Morrison, 2010). The Administration for Children and Families (2012) reported that the incidence of maltreatment occurrences increased for caregivers with the following risk factors: alcohol abuse, drug abuse, and domestic violence. In fact, parents involved in relationships in which intimate partner violence is present have repeated reports of maltreatment at twice the rate of those in non-violent domestic environments (Casanueva, Martin, \& Runyan, 2009). Additional risk factors include living in poverty and impoverished neighborhoods (Wulczyn, 2009), maternal depression (Casanueva, Cross, Ringeisen, \& Christ, 2011), lack of social support (Kotch, Browne, Ringwalt, Dufort, Ruina, Stewart, \& Jung, 1997), high stress (Wulczyn, 2009; Kotch et al., 1997), and single parenthood and large family size (Bae et al., 2009). Inherent in these risk factors may be the caregiver's inability to be attuned to the needs of the child, particularly when combined with the child's individual-level risk factors such as developmental delays. These proximal processes from the caregiving environment may be compromised, thus negatively impacting child outcomes. This may be particularly true for the development of effective emotion regulation skills, language, and school readiness.

Protective factors that serve to buffer children against the negative effects of maltreatment include high quality parent-child relationship, whether the parents are birth parents or substitute caregivers such as foster parents. A study by Alink, Cicchetti, Kim, and Rogosch (2009), examined the mediating role of parent-child relationship quality on behavior problems. 
The authors found that positive parent-child interaction was a significant mediator in lowering maltreated children's internalizing and externalizing symptoms. More specifically, the authors noted that secure relatedness to their mothers was associated with children's better emotion regulation skills. Additional caregiver-related protective factors include positive family functioning defined as the well-being or performance of the family unit; emotional support defined as the individual's perception that support will be provided by their social network; concrete or instrumental support defined as the tangible resources such as food, cash, child care assistance, and clothing; and nurturing and attachment defined as the level of positive relationship between parent and child (Counts, Buffington, Chang-Rios, Rasmussen, \& Preacher, 2010).

The biological home as the primary microsystem of development has been documented to be a source of early adversity because of children's experience of maltreatment or witnessing violence. However, it can also serve as a context for positive development when positive proximal processes are in place (Alink et al., 2009; Haskett, Stelter, Proffit, \& Nice, 2012). It is important to note, however, that a shift in parent-child interaction quality from negative to positive cannot occur as a one-time event. The next section will discuss the importance of consistency and regular interaction for these positive changes to occur and be maintained.

\section{Chronosystem-level Factors}

The length of time needed for developmental outcomes to come to fruition is important when taking into account how consistent and stable the interaction between a child and her/his environment is (Bronfenbrenner \& Morris, 2006). For children with a history of maltreatment, the chronosystem-level influences appear to be nested within their microsystem contexts of biological or foster home and the school environment. Of particular importance are stability and 
duration of placement given the negative outcomes related to increased duration of placement in foster care (i.e., lack of permanent caregivers), as well as the negative outcomes related to shorter stay due to instability (i.e., lack of stable caregivers; Jones Harden, 2004; Lloyd \& Barth, 2011; Ryan \& Testa, 2005; Stacks \& Partridge, 2011). A study by Wulczyn, Kogan, and Jones Harden (2003) demonstrated that children in foster care will experience multiple placement changes and that instability is likely to occur within the first 6 months of placement. Placement instability has been indirectly related with negative school outcomes through increased school mobility (Altschuler, 1997; Yu, Day, \& Williams, 2002). Additionally, the negative academic outcomes associated with child welfare entry, multiple placements, and school changes have been documented (Smithgall, Gladden, Howard, George, Courtney, 2004). These variables represent instability in the lives of maltreated children in the child welfare system. Without stability, it is difficult for positive adult-child interactions to occur. It is only over time that proximal processes, the most important mechanisms for development, can exert influence on the development of children within their contexts.

\section{Process-level Factors}

As mentioned earlier, bio-ecological theory proposes that proximal processes are the primary mechanisms that lead to developmental outcomes. Teachers and caregivers are present in the immediate environment (i.e., microsystems) of maltreated children and are in a position to promote development through the quality of their interactions. Teachers have a major role in promoting positive academic outcomes whereas caregivers are particularly instrumental in promoting the development of emotion regulation and language, though both these adults can contribute to these outcomes. These positive interactions need to occur consistently over time, which underscores the importance of maintaining home and school placement stability. 


\section{Parent/Caregiver-Child Relationship}

The quality of the caregiver-child relationship, whether referring to biological, foster or kinship caregivers, is an important process for promoting positive outcomes in children (Cristofaro \& Tamis-LaMonda, 2011; Healey \& Fisher, 2011). For children with a history of maltreatment, the relationship qualities related to emotional support and cognitive stimulation are especially important in promoting emotion regulation and language development. For example, Stacks and Partridge (2011) conducted an analysis using NSCAW data to examine the developmental needs of infants who enter foster care and to assess if their needs differed by placement type, as well as the quality and quantity of stimulation and support in the home. They found that the quality of the foster and kinship placement homes and neighborhoods differed in that foster homes provided a higher quality home environment. More specifically, foster care placements scored higher than kinship placements on measures of cognitive stimulation such as more books in the home and increased reading time. With regard to emotional support, kinship placements appeared to score higher on hostile interactions with their infants when compared to foster care parents.

Further evidence that emotional support and cognitive stimulation provided within the context of the parent-child relationship was provided by Jones Harden and Whittaker (2011). In their study looking at the infant sample from the NSCAW data, the authors found that cognitive stimulation did not affect cognitive development for younger children. However, for older children, waves 1 and 4 cognitive stimulation was found to positively affect cognitive development within their respective waves. For young children, wave 1 cognitive stimulation was associated with lower behavior problems and higher levels of wave 4 cognitive stimulation. Wave 4 cognitive stimulation was also negatively related to behavior problems for older 
children. Wave 1 cognitive stimulation was also positively associated with social skills at waves 1 and 3. With regard to emotional support, the authors did not find any significant associations with cognitive development for young children. However, significant associations were found for older children in that wave 3 emotional support contributed to cognitive development. In addition, wave 4 emotional support contributed to language development. No further associations were found for older or younger children's behavioral and social skills. These results point to the importance of caregiver emotional support and cognitive stimulation for maltreated children's developmental outcomes. Further, it appears that both types of caregiver quality have positive effects across domains.

Evidence for the important role of the caregiver-child interaction in promoting healthy outcomes can be inferred from evidence-based practices (EBPs) that clearly highlight this proximal process as the mechanism for change in parenting interventions. One example of promising EBPs within child welfare is Parent-Child Interaction Therapy (PCIT). PCIT was designed to address a broad range of behavioral, emotional, and family interaction problems and to be a short-term, evidenced-based intervention for families with preschool-aged children between the ages of 2 and 6 (Herschell, Calzada, Eyberg, \& McNeil, 2002). In a randomized controlled trial conducted by Thomas and Zimmer-Gembeck (2011), the authors found that mothers with a history of maltreating their preschool-aged children, when compared to a waitlist, showed improved parent-child interactions, better child outcomes, and decreased stress after 12 weeks of participation. Moreover, at the completion of the entire PCIT curriculum, the authors found that mothers reported less child abuse potential and increased maternal sensitivity. Similar results were found by Timmer, Urquiza, Zebell, and McGrath (2005) in their study that compared a sample of maltreatment parent-child dyads with non-maltreatment dyads. These 
families had children between the ages of 2 and 8 years old with a mean age of 4.58 years. The authors found that at the completion of the PCIT training, there were reports of decreased child behavior problems, decrease in parental stress, and decrease in abuse risk for participants with a history of maltreatment.

Finally, a study conducted by Chaffin, Silovsky, Funderburk, Valle, Brestan, Balachova, Jackson, Lensgraf, and Bonner (2004) investigated the efficacy of PCIT in reducing future abuse reports of physically abusive parents. In their study, the authors conducted a randomized trial that compared three treatment conditions: PCIT, PCIT plus individualized enhanced services, and standard community-based parenting group. Children were between the ages of 4 and 12 years. The authors found that compared to the community parenting group (49\% re-report), those parents enrolled in the PCIT-only condition showed a significant decrease in re-report in physical abuse (19\% re-report). They added that the enhanced services condition did not improve the efficacy of PCIT and thus attributed the reduction of negative parent-child interactions to the efficacy of PCIT. Due to the use of randomized trials, these studies that examined the efficacy of a PCIT intervention can provide evidence for a causal relationship between parenting quality and child outcomes.

Taken together, the literature reviewed in this chapter point to the importance of emotion regulation for children's school readiness and later academic achievement. In addition, experiencing maltreatment has been shown to negatively impact developmental trajectories of young children. Despite the emerging research that examined the relationship between emotion regulation, language, and maltreatment, empirical attention to this area is still limited. Most of the studies reviewed above have focused primarily on children in foster care. Further research is needed to understand how emotion regulation is affected by children who remain in their homes. 
For these children, multiple placements often occur that include reunification and removal, which indicates high caregiving instability. Further, these children show the highest rates of absenteeism after reunification (Zorc et al., 2013). This concern is more salient for preschoolaged children given the higher likelihood of remaining in their home after a CPS investigation (Stahmer et al., 2005).

\section{Gaps in the Literature}

Based on the discussion so far, it is clear that maltreatment experiences involves a complex interaction of multiple ecologies for the affected child. Given the overlap and interaction between ecological systems, it is apparent that future research, intervention, and policy should take into account the multi-systemic nature of maltreatment for addressing change for affected children. For example, employing the full Person-Process-Context-Time (PPCT) model may allow a more accurate view of these complex systems and processes that influence the development of maltreated children's school readiness.

With regard to maltreatment type, there is growing evidence that antecedents and developmental outcomes vary based on the maltreatment classification placed upon a child's experience. However, the true experience of maltreatment type is challenging to measure based on lack of specificity in the current instruments. Additionally, it is often difficult to parse out the variance between developmental outcomes that are the result of maltreatment versus other causes such as risk factors related to the onset of maltreatment. However, future studies should incorporate exploratory analyses to examine how classification might provide insight in different outcomes. These studies could then be followed up with a line of inquiry that specifically examines the reliability and validity of maltreatment subtypes. 
Jones Harden and Klein (2011) provided several recommendations for research that could inform child welfare practice about the nature of maltreated children's development and interventions to potentially support positive changes, through promoting well-being in addition to improving safety and permanency. Relevant to this review are the following recommendations:

Exploring the unique child welfare ecologies of different stages of development. Further research studies could specifically include several microsystems within a child's mesosystem that interact to promote development. Additionally, the longitudinal analysis of outcomes nested within contexts could allow a better understanding of stage-salient differences throughout development. For example, studies can employ both foster homes and families of origin as microsystems that interact with the child's school environment. These interactions offer a unique mesosystem for the child, which provide differential level of attention based on age of involvement (i.e., preschool or school-age), as well as children with different experiences of early attachment and language stimulation.

Examining core developmental processes. Based on the evidence presented, inclusion of accurate and sensitive measures to assess emotion regulation and language development are important in further understanding how each of these core developmental process can individually and interactively mediate later academic readiness. Reducing measurement errors can also provide important tools to implement a Response to Intervention (RTI) approach that could tailor effective interventions to maltreated children who display specific school readiness profiles. An RTI approach provides more accurate and effective interventions to help promote better school readiness skills through early identification and differentiating instructions for children who display deficits in emotion regulation or language skills. RTI usually includes 
universal screening to identify children who need additional school support; ongoing assessment of children's progress over time, increasing frequency of services for those who display increased needs; multiple tiers of support; and a decision-making model so that children identified with weak and very weak skills receive more intensive support in a timely manner (Greenwood, C. R., Carta, J. J., Atwater, J., Goldstein, H., Kaminski, R., \& McConnell, S., 2012).

One example of a methodological approach to specify school readiness profiles is the use of person-centered designs such as finite mixture models. These methods could shed some light on possible heterogeneity in emotion regulation and language development profiles for children who have maltreatment histories. Intervention and assessment can then be provided based on these specific profiles. Additionally, future studies should include the role that gender plays in language and emotion regulation development.

Conducting studies of long-term developmental outcomes. Academic outcomes during school age and adolescence could be better understood by examining earlier processes associated with school readiness. Application of prospective longitudinal designs and analyses may provide information on growth trajectories and sequelae of deficits documented during baseline analysis. Investigating the processes relating to the quality of family functioning. This allows for the examination of proximal processes important in promoting resilience or risk with respect to child outcomes. Due to the complex contextual considerations for children involved with child welfare, processes related to the quality of family functioning should include other microsystems as well. For example, related (kinship) and non-related (fictive kinship) foster placement, traditional foster placement, and biological homes of children should be investigated. It is 
particularly important to understand the relationship between family functioning and development of emotion regulation skills and language.

\section{Concluding Remarks}

Negative developmental outcomes have been associated with experiencing maltreatment early in life. Numerous studies have examined the physical and mental health of maltreated children, with little attention paid to academic outcomes. Through the framework proposed by the bio-ecological model, these academic outcomes have been shown to be influenced by proximal processes that connect biological/individual and ecological factors specific to children who have experienced maltreatment. Understanding the role that these person-context-timeprocess variables play in promoting development, particularly in early childhood, can provide directions for future research to further understand experiences unique to maltreated children. This in turn can help inform policy and practice to facilitate the academic success of this vulnerable population. 


\section{Chapter 3: Methods}

This study examined the emotion regulation/dysregulation development of young maltreated children over 3 waves of data collection (i.e., $3-5$ years old at wave $1,4 \frac{1}{2}-6$ 1/2 years old at wave 3, and 6-8 years old at wave 4). Additionally, heterogeneity in groups based on developmental patterns was examined. Next, language, gender, placement instability, caregiver emotional support, and caregiver cognitive stimulation were considered for their influence on the development of emotion regulation/dysregulation in young maltreated children. Finally, this study explored the relationship between emotion regulation/dysregulation developmental patterns and later academic achievement. The National Survey of Child and Adolescent WellBeing (NSCAW) restricted release data was employed in order to answer the research questions. This chapter provides an outline of the NSCAW dataset, the measures used, and the analytic plan for answering the research questions.

\section{The National Survey of Child and Adolescent Well-Being}

The National Survey of Child and Adolescent Well-Being (NSCAW) was the first nationally representative, longitudinal survey of children and families who were investigated by Child Protective Services (CPS). It was also the first national study to relate family characteristics, child welfare system experiences, community environment, and other factors to child and family well-being. The NSCAW study was a fixed panel design with 3 waves of data collection wherein children selected into the sample at baseline in 1999 and 2000 were followed over time. Data were then collected at approximately 18 months (wave 3) and 36 months (wave 4) after baseline (see Table 1). Current caregivers, caseworkers, and teachers were also surveyed across all three waves. Wave 2 included caseworkers and caregivers who were surveyed approximately 12 months after baseline. These surveys did not include measures of well-being 
and functioning similar to waves 1 and 3 and therefore are not considered as a wave of data collection in the subsequent analyses. A fifth wave of data collection was later included in order to examine long-term outcomes. Data from wave 5 were collected between September 2005 and December 2007, and were fielded by age cohort rather than time interval since investigation. Children in the infant cohort (0-12 months at the time of sampling) were fielded in September 2005-February 2006 (3-5 years at wave 5). Children aged 12-48 months at baseline were fielded next around February-November 2006. Young adults (18 years old by April 2006) were fielded in July 2006. The remaining children were fielded between March and December, 2007. Table 1

Timeline of NSCAW Data Collection

\begin{tabular}{cccccc}
\hline \multicolumn{6}{c}{ Wave } \\
\hline \multirow{2}{*}{ Start and End Dates } & 1 & 2 & 3 & 4 & 5 \\
\cline { 2 - 6 } & $\begin{array}{c}11 / 15 / 1999- \\
04 / 20 / 2001\end{array}$ & $\begin{array}{c}10 / 01 / 2000- \\
03 / 31 / 2002\end{array}$ & $\begin{array}{c}04 / 01 / 2001- \\
09 / 30 / 2002\end{array}$ & $\begin{array}{c}08 / 01 / 2002- \\
02 / 28 / 2004\end{array}$ & $\begin{array}{c}09 / 05 / 2005- \\
12 / 30 / 2007\end{array}$ \\
\hline $\begin{array}{c}\text { Months after Close of } \\
\text { Investigation }\end{array}$ & $2-6$ & 12 & 18 & 36 & $59-97$ \\
\hline Child & \multicolumn{2}{c}{ Respondent } & & & \\
\hline Current Caregiver & $\mathrm{X}$ & $\mathrm{X}$ & $\mathrm{X}$ & $\mathrm{X}$ & $\mathrm{X}$ \\
Investigator/Services & $\mathrm{X}$ & $\mathrm{X}$ & & & \\
Caseworker & $\mathrm{X}$ & $\mathrm{X}$ & $\mathrm{X}$ & $\mathrm{X}$ & $\mathrm{X}$ \\
Teacher & $\mathrm{X}$ & & $\mathrm{X}$ & $\mathrm{X}$ & $\mathrm{X}$ \\
\hline
\end{tabular}

Source: NSCAW Data File User's Manual (DFUM; 2008)

The study cohort included 6,228 children from birth to 14 at the time of sampling. These children were selected from two groups, the Child Protective Services (CPS) and the longer-term foster care (LTFC) samples. The CPS sample included 5,501 children who were the subject of child abuse or neglect investigations at the time of sampling (October 1999 - December 2000). The LTFC sample had 727 children who were under investigation for child abuse and neglect and living in out-of-home care for approximately one year at the time of sampling. Both groups 
were selected from 92 Primary Sampling Units (PSUs) in 97 counties across the country. The sample included cases that received on-going services and cases that did not receive services. For the latter, services were not continued due to investigations not being substantiated (i.e., lack of evidence to support maltreatment occurrence) or because of determination that services were no longer needed. Interviews or assessments at baseline, wave 3, wave 4, and wave 5 were conducted face-to-face with children, parents or permanent caregivers, non-parent adult caregivers such as foster caregivers, teachers for school-aged children, and child welfare investigators.

\section{Target Population and Sample Design}

The target population in the CPS sample included all children in the United States who were subjects of child abuse or neglect investigations conducted by CPS investigators and who lived in states not requiring agency first contact. Four states were excluded from the study because the laws required that the first contact with a caregiver whose child was selected for the study was made by CPS staff instead of an NSCAW field representative.

The study used a two-stage stratified sample design in order to select the NSCAW sample. During stage 1, the United States was divided into nine sampling strata where 8 of the strata corresponded to the 8 states with the largest child welfare caseloads (i.e., California, Florida, Illinois, Michigan, New York, Ohio, Pennsylvania, and Texas). The ninth stratum consisted of the remaining 38 states and the District of Columbia. During stage 2, Primary Sampling Units (PSUs) were formed and selected within each of the 9 strata. The PSUs were defined as geographic areas that surround a population area served by a single CPS agency. The sample PSUs were randomly selected using a probability-proportionate-to-size (PPS) procedure, which gave a higher chance of selection to PSUs with larger caseloads. 
The NSCAW PSU sample frame was composed of all counties in the U.S. that were large enough to support one interviewer workload with about 60-67 cases per year. Counties that were smaller than this size were deleted from the sampling frame. Each within-PSU sampling strata was comprised of eight mutually exclusive and exhaustive categories of children (i.e., sampling domains). To select the sample of PSUs, each PSU in the population was assigned a size measure that was a function of the desired sampling rate for each of the eight sampling domains within each stratum and the estimated target population size in each PSU.

Using systematic sampling, an independent sample was drawn from each stage 1 stratum. Sorting the first stage frame before sample selection resulted in implicit stratification. The original sample consisted of 100 PSUs and after selection, 7 were deemed too small and were combined with adjacent counties, 6 refused and replaced with similar sized PSUs, and 8 were determined to be ineligible. The final sample consisted of 92 responding, eligible PSUs.

The eight within-PSU sampling domains were used for selection of the NSCAW CPS sample. This study was interested in examining the individual-level and context-level factors that impact emotion regulation development for young maltreated children after first contact with CPS. Given the interest in early childhood, caregiving quality between the family of origin and short-term foster care, and placement stability, this study only drew from the CPS sample. Though important in understanding developmental processes of children in long-term foster care, the LTFC sample included children who have been in out-of-home placement for over a year at the beginning of baseline and may not include the necessary sample size to answer questions that related to early childhood. Further information on the LTFC sample is available in the Data File User's Manual (DFUM; 2008). The domain structure consisted of the cross-classification of 4 child characteristics (see Appendix A). The first level divided children into 2 groups: not 
receiving services (group A: Domains 1 and 2) and receiving services (group B: Domains 3-8). For group A, two subdomains included children who were less than 1 (Domain 1) and older children (Domain 2). Group B was further divided into 6 subdomains: by age (under 1 year old and 1-14 years old), then within each age subgroup, by placement setting (in-home care and outof-home care). The older subgroup by placement setting was then further divided by type of abuse/neglect (investigated for sexual abuse allegations then all others).

The sampling process occurred over a 15-month period and, as stated earlier, included all children investigated or assessed for child abuse and neglect between October 1999 and December 2000. Participating agencies identified eligible children ages 0 to 14 and provided relevant files each month. Children 15 years old and older were excluded and removed from the sampling frame. There was an oversampling of infants, sexual abuse cases, and cases receiving ongoing services after investigation. Sample age was capped at 14 years in order to increase the likelihood that children could be located due to challenges once children emancipate. The sampling approach allowed for generation of national estimates for the full population of children and families who entered the child welfare system, as well as adequate power to consider subgroups within the child welfare population.

To avoid duplication or resampling, children who were identified from the previous month were deleted from the current month's submission. Additionally, children who were members of the same household that was previously identified were removed from the current month in order to limit increased burden on the families. Children under investigation as perpetrators of the abuse were ineligible and thus excluded from the sample. Once the inclusionary and exclusionary criteria were applied, a simple random sample of children was selected from within each of the sampling domains. 
Sampling rates were adjusted as necessary at the end of baseline data collection and number of interviews in each domain were kept as close to the target sample size as possible. Adjustments to the sampling rates were monitored and conducted in order to keep workload in each PSU within an acceptable range and to keep the domain-specific unequal weighting effect as small as possible for each PSU. All children with a completed interview at wave 1 were followed up and contacted for participation in subsequent waves.

\section{Bias Due to Sampling Frame Non-coverage Error}

Based on the sampling frame discussed above, there should be a positive probability that every member of the target population will be selected. However, inclusion of every child referred to CPS is impractical, and differences in maltreatment definitions across states may change the selection rate of children. When these children from the target population are missing from the sample frame, the members they represent have a low probability of selection, which results in a frame non-coverage error. The DFUM (p. 2-9) includes details on calculation of noncoverage error rates. According to the manual, bias due to frame non-coverage is comprised of (1) the non-coverage rate, and (2) the difference in mean characteristic for the subpopulation covered and the subpopulation not covered by the sampling frame. That is, a high non-coverage rate will relate to a large non-coverage bias if the difference in mean characteristic is not small. If, on the other hand, this difference in mean characteristic is small, the non-coverage bias may be small regardless of the non-coverage rate.

Several types of non-coverage exist in the NSCAW frame. First, counties or agencies with few children investigated for maltreatment were deleted. Second, PSUs in a few states were removed because first contact with CPS (designated as "first contact sites") was required instead of NSCAW representatives. Because response rates from these PSUs were so low, they were 
removed from the frame and the target population was redefined to reflect the exclusion of states that required CPS agency contact first. Finally, some agencies did not provide records for all the children investigated for abuse or neglect due to incomplete records or delay in data entry. These types of non-coverage errors may cause population estimate bias.

To account for these potential biases, the NSCAW data manual (DFUM, 2008) provided detailed analyses on each of these types of non-coverage and only the final results are provided here. For bias due to eliminating small counties, 710 out of the 3,141 ( 23\%) counties were excluded from the sample. It was estimated that less than $3 \%$ of the target population resided in these counties and that their exclusion would not have significant consequences on statistical inference.

For bias due to removing first contact sites, no adjustment to the estimates for the missing states were conducted in the sample weights. These first contact site states were excluded from the NSCAW inferential sample and the manual recommends that inference from the data should reflect this exclusion. Strictly speaking, inference beyond "all children in the United States who were subjects of child abuse or neglect investigations conducted by CPS investigators and who live in states not requiring agency first contact" may not be valid. However, further analyses revealed that the NSCAW estimates may still be nearly unbiased estimates of the entire U. S. population (including the first contact states). According to the manual, non-coverage bias due to the exclusion of first contact sites was ignorable since the proportion of the total child welfare population in these states is less than $5.2 \%$.

For bias due to record completeness, the NSCAW study team compared the completed surveys with county data on the number of children investigated for maltreatment during the reporting period. Using the procedures outlined in the manual, egregious errors were identified 
and non-coverage errors were prevented. It was concluded that cases were missing at random. Further analyses were conducted to examine the distributions of demographic characteristics to verify the missing at random data structure. Results revealed that the distributions of demographic characteristics were closely similar across the weighted sample and the agency data. Adjustments were made in order to assure that any residual bias in the NSCAW data was minor.

\section{Sampling Weights}

The NSCAW sample design includes complex features such as unequal selection probabilities, stratification, and clustering. According to Stapleton (2002), traditional and modern analytic techniques operate on assumptions about the sample design. If these assumptions do not hold, then incorrect estimation of key parameters and their variances may result, which compromises hypothesis tests and inferences drawn from the results. This section will briefly outline problems with parameter estimates if complex sampling design is not taken into account during model specification. Unequal probability sampling assumes that elements in the population are associated with unequal selection probabilities when sampled at different rates. If these unequal probabilities are ignored, then biased parameter estimates known as selection bias may result when the probability of selection is correlated with the response variable (Asparouhov, 2005; Stapleton, 2002). Inclusion of design weights can compensate for differential nonresponse and reflect the original unequal sample inclusion probabilities (Stapleton, 2002). Stratification refers to the division of all elements in the sampling frame into mutually exclusive categories where sampling is performed within each category (Stapleton, 2006). The division of each stratum into PSUs within is an example of stratification in the sampling design for the NSCAW study. More specifically, the NSCAW study employed 
disproportionate sampling, which means that the use of sample weights is required to obtain unbiased parameter estimates (Stapleton, 2006). Clustering sampling designs often include a degree of dependence among observations, violating an assumption in traditional analyses that observations are independent and identically distributed (Stapleton, 2002). If the level of dependence is not accounted for, then the sample variance may be underestimated, which results in high levels of Type I error rates (Stapleton, 2002).

The NSCAW data contain several weight variables that reflect differential selection probabilities of sample members, which account for under-coverage and nonresponse from the final sample. These weights are essential when employing a longitudinal analysis in order to obtain approximately unbiased estimates of the population parameters. Because response patterns vary by wave, different weights for each wave was required during analyses.

Additionally, coverage adjustments were made on the sample frames (i.e., CPS and LTFC) due to missing cases. When considering weight requirements, selection of different weights are needed based on the level of analysis (e.g., national or stratum level). Descriptions of the calculations for the CPS weights are provided in Section 7 of the NSCAW Data File User's Manual (2008) and Section 1 of the NSCAW Statistical User's Manual (2008).

Analysis weights were constructed in stages corresponding to the stages of the sample design (i.e., first stage PSU weight and initial sampling base weight). Adjustments were made due to missing months of frame data or types of children, nonresponse, and under-coverage. NANALWT weights were created for making inference at the national level, which includes inference for substantiated and unsubstantiated cases. SANALWT weights were created for making inferences at the stratum level. All children were followed across baseline, wave 2, wave 3, wave 4, and wave 5. At wave 2, interviews with current caregivers and caseworkers were 
attempted for all the children from wave 1 . At waves 3, 4, and 5, interviews with current caregivers, caseworkers, and children were attempted. Nonresponse was incurred in waves 2 through 5, which have the potential for nonresponse bias estimates for the later waves. Adjustments to the wave 1 weights were made to compensate for these nonresponses at later waves. Weights were created for children who responded to waves 1, 3, 4 and waves 1, 2, 4, 5 . See Appendix B for available sampling weights and types of analyses appropriate for each constructed weight. Given the interest in national-level parameters across all 5 waves of data collection, multiple national-level weights were selected based on the analytic procedures used.

Standard error and chi-square tests of model fit corrections were accounted for when the appropriate weight variable was selected. Within Mplus, incorporation of sampling weights was accomplished by using the TYPE=COMPLEX option of the ANALYSIS command in conjunction with the STRATIFICATION, CLUSTER, and WEIGHT options of the VARIABLE command. This study used the NSCAW Restricted Release version, which included geographic details and fewer recoded variables. Access to the Restricted Release required additional steps and requirements to ensure that data access was limited to research personnel.

\section{Measures for the Proposed Study}

This section will include details on the selected measures used as indicator variables for emotion regulation. Additionally, covariate variables and the distal outcome of academic achievement will be discussed. Each section will begin with an overview of the derived variables for the study. This will be followed by a general description of the measures from the NSCAW study, along with psychometric properties for each measure. Psychometric properties for each measure were taken from the Data User's File Manual (2008), the Statistical Analysis User's Manual (2008), and the Instrumentation Manual (2008) from NSCAW. 


\section{Emotion Regulation/Dysregulation}

The NSCAW dataset did not contain any direct measure of emotion regulation/dysregulation for children beyond 4 years old. Using the process model of emotion regulation, a factor-derived measure was conducted in order to construct a measure for emotion regulation/dysregulation.

According to the theoretical model presented in Chapter 2 on emotion regulation, the latent factor for the study included subscales derived from the CBCL (2-3 and 4-18; Achenbach, 1991) and a subscale from the Social Skills Rating System (SSRS; Gresham \& Elliot, 1990). The Anxious/Depressed, Attention, and Aggression subscales, also known as the AAA profile, from the CBCL has been shown to be indicative of emotion and behavioral dysregulation (Althoff, 2010; Ayer et al., 2009; Spencer et al., 2011) and procedures outlined in these studies were replicated in order to arrive at the indicator variables for the emotion regulation/dysregulation latent factor. The process model of emotion regulation was used as a framework to select response items from the CBCL and SSRS as indicators of emotion regulation/dysregulation.

In addition to the AAA profile from the CBCL, the Self-control subscale of the Social Skills Rating System (SSRS; Gresham \& Elliott, 1990) was included as indicator variables for the emotion regulation latent factor. Based on the theoretical perspective presented in Chapter 2, inclusion of this subscale appears valid. Inhibitory control, a component of emotion regulation specifically, and self-regulation broadly, share common components that define self-control. Sample items include, "controls temper in conflict situations with you, responds appropriately when hit or pushed by other children, and controls temper when arguing with other children."

Ayer et al. (2009) speculated that the AAA profile, along with another cluster of CBCL syndrome scores, represented a latent factor that they hypothesized as a dysregulatory system. 
The authors recommended that replicating their findings or incorporating other factors related to emotion regulation may provide further validity to their results. Inclusion of the self-control subscale may validate these findings.

Child Behavior Checklist (CBCL; Achenbach, 1991). This study employed two versions of the CBCL, measures for ages 2-3 and 4-18. The CBCL is typically administered to parents, teachers, and children to obtain a report on competencies and behavior problems. The CBCL 2-3 includes 9 scales: 6 syndrome scales (anxious/depressed, sleep problems, aggressive behavior, withdrawn, somatic problems, \& destructive behavior) and 3 compiled scales (internalizing problems, externalizing problems, \& total problems). Test-retest reliability show medium to high reliability for the syndrome scales $(\alpha=0.72-0.93)$, the internalizing scale ( $\alpha$ $=0.87)$, the externalizing scale $(\alpha=0.84)$, and the total problems scale $(\alpha=0.91)$. Construct validity was $0.56-0.77$ when compared with the Richman Behavior Checklist. Internal consistency for the NSCAW sample was high for externalizing $(\alpha=0.91)$, internalizing $(\alpha=$ 0.80), and total problem behaviors ( $\alpha=0.95$ ). Children who scored above 60 on the externalizing, internalizing, and total problem behaviors were classified as having clinical/borderline problem behaviors.

The CBCL 4-18 includes 8 syndromes: social withdrawal, somatic complaints, anxiety/depression, social problems, thought problems, attention problems, delinquent behavior, and aggressive behavior. In addition, the CBCL 4-18 includes a sex problems scale. There are 2 broad groupings of syndrome scales: externalizing and internalizing scales. The internalizing problems scale combines the social withdrawal, somatic complaints, and anxiety/depression scales. The externalizing problems scale combines the delinquent behavior and aggressive behavior scales. The NSCAW Instrumentation Manual reports high internal consistency and test- 
retest reliability. The manual also reports high construct validity when correlated with similar scales corresponding with psychopathology diagnoses from the Diagnostic and Statistical Manual of Mental Disorders (4 ${ }^{\text {th }}$ ed., text rev.; DSM-IV-TR; American Psychiatric Association, 2000). Internal consistency for the NSCAW sample was high for externalizing ( $\alpha=0.92$ ), internalizing ( $\alpha=0.90$ ), and total problem behaviors $(\alpha=0.96)$. Children who scored above 60 on the externalizing, internalizing, and total problem behaviors were classified as having clinical/borderline problem behaviors.

Item responses for both the CBCL 2-3 and CBCL 4-18 are on 3-point scale ranging from 0 (not true), 1 (somewhat or sometimes true), and 2 (very or often true). Item responses are summed up to arrive at a raw score. Summary raw scores are calculated for each of the syndromes, the internalizing, externalizing, and total problems scale. Standardized scores are also available for each of the scales.

Social Skills Rating System (SSRS; Gresham \& Elliott, 1990). The SSRS measures social competence using three versions: preschool, elementary, and secondary versions. A majority of the items describe positive social behaviors. There are 40 items in the SSRS under 2 domains of social competence: social skills (30 items) and problem behaviors (10 items). The social skills domain includes cooperation, assertive, responsibility, and self-control subscales while the problem behaviors domain includes externalizing and internalizing subscales. The SSRS has 3 forms (i.e., parent, teacher, and student), which have all been employed in the NSCAW study. Each form has the same domains and subscales except for the addition of an academic competence scale with the teacher form and the empathy subscale for the student form. Item responses are on a 3 -point Likert scale: $0=$ never, $1=$ sometimes, and $2=$ very often. 
The caregiver version was selected for the current study. The parent rating is psychometrically sound with internal consistency $\alpha=0.73-0.95$ and test-retest reliability $\alpha=$ $0.65-0.87$. The SSRS shows criterion validity for the parent form, when compared with the CBCL social skills scale $(r=0.58)$. Finally, internal consistency for the NSCAW sample was high for preschool and secondary $(\alpha=0.90)$ and elementary $(\alpha=0.87)$.

\section{Covariates}

Early childhood is an important period in the development of school readiness skills, particularly in the socioemotional domain (Kagan et al., 1995). Being ready for school during early childhood can help children transition successfully into formal schooling (Duncan \& Magnuson, 2011; McWayne et al., 2012). Development of school readiness skills, especially socioemotional skills, rely on the transactional processes between individual-level and contextlevel factors. Based on the studies reviewed in Chapter 2, however, young maltreated children who come into contact with CPS are at an increased developmental and contextual risk that impact the development of their school readiness. Thus, this study included individual-level factors (i.e., gender and language) and context-level factors (i.e., placement stability, caregiver cognitive stimulation, and emotional support) in order to understand how these covariates impact emotion regulation development across time. These covariates were taken from wave 1 and employed as time-invariant (i.e., having an effect across time). Placement stability scores were calculated for this study and procedures for this derived stability index, as well as descriptions for the other covariate measures will be discussed next.

Language. The language scores were taken from the Preschool Language Scale - 3 (PLS-3; Zimmerman, Steiner, \& Pond, 1992) for children under 6 at wave 1 . The PLS-3 is an instrument used to evaluate language development and tests receptive and expressive language 
skills. Additionally, the PLS-3 is used to assess behaviors that are considered to be precursors to language development. The standardized scales are auditory comprehension (AC), expressive communication (EC), and a total language score. The total language score includes pre-linguistic skills (attention, vocal development, and social communication) and language skills (syntax, morphology, vocabulary, concept development). Children are directly observed and assessed by trained individuals and response items are scored by entering a check for each correct response and a minus for each incorrect response. The PLS-3 yields standard and age-equivalent scores for all 3 scales. Internal consistency for the EC, AC, and total language scores are $r=0.84,0.79$, and 0.88 respectively. Test-retest reliability for the scales is as follows: $\mathrm{EC}=0.82-0.92, \mathrm{AC}=$ $0.89-0.90$, and total language $=0.91-0.94$. For construct validity, the manual reports that the PLS-3 discriminates language disordered children $66 \%-80 \%$ of the time. Concurrent validity with the PLS-Revised is $r=0.86$ for the EC scale, $r=0.66$ for the AC scale, and $r=0.88$ for the total language score.

Placement stability. Placement stability scores were calculated following recommendations from Casanueva, Dozier, Tueller, Dolan, Smith, Bruce Webb, Westbrook, \& Jones Harden (2014). The authors defined stability, or caregiver instability, as "a new placement for one week or longer in a different household and/or with a new caregiver" (p.499). A change in caregiver was counted if a child was placed in a new home for 7 days or more and the original caregiver did not move along with the child. Placement at baseline was counted as " 0 " to reflect no change in placement, which would include foster care or biological family.

Caregiver emotional support and cognitive stimulation. The Home Observation for Measurement of the Environment (HOME) inventory (Caldwell \& Bradley, 1984) was used to measure caregiver emotional support and cognitive stimulation. The HOME inventory was 
designed to measure the quality of stimulation and support available to a child in their home environment. Information is gathered through observation and interview of the primary caregiver. Scores were calculated based on the answers to the semi-structured interview questions and observations of the home by trained personnel. The measure yields total and subscale scores for the preschool (3-5 years old) and elementary (6-10 years old) sample. Subscale items include learning stimulation, language stimulation, physical environment, warmth and affection, academic stimulation, and modeling. Internal consistency (i.e., $\alpha$ ) for the subscales was $0.53-0.88$ and 0.93 for the total score. Predictive validity was reported as being associated with measures of language development and success in school. Internal consistency for the NSCAW sample was reported as $\alpha=0.41$ for emotional support and $\alpha=0.71$ for physical environment for the preschool sample. Internal consistency for the elementary sample was $\alpha=.48$ for cognitive stimulation, $\alpha=0.48$ for emotional support, and $\alpha=0.74$ for physical environment.

\section{Distal Outcome}

The Woodcock-Johnson III Tests of Achievement (WJ-III ACH; Woodcock, McGrew, \& Mather, 2001) at wave 5 was selected as the distal outcome for this study. The WJ-III ACH includes 22 tests for measuring skills in reading, math, writing, oral language abilities, and academic knowledge. The WJ-III ACH provides norm-referenced measures of academic abilities with each of the tests measuring one or more specific abilities. Only 4 of the 22 tests were included in the NSCAW study: letter-word identification, calculation, passage comprehension, and applied problems.

Passage comprehension requires the construction of propositional representations, integration of syntactic and semantic properties of printed words onto a representation of the 
entire passage, and inferential bridging. The applied problems test requires construction of mental mathematics models through language comprehension, application of math knowledge, calculation and quantitative reasoning, and insight. Letter-word identification is concerned with feature detection and analysis of letters, as well as the recognition of visual word forms and phonological access to pronunciations. Calculation tests math achievement with cognitive processes that access and apply knowledge of numbers and calculation procedures, as well as verbal associations between numbers represented as words (Wendling, Schrank, \& Schmitt, 2007).

The following are split-half reliability values for the subtests used in this study: applied problems test $(r=0.93)$, passage comprehension test $(r=0.88)$, letter-word identification $(r=$ 0.94), and calculation $(r=0.86)$. Concurrent validity (i.e., $r$ ) evidence showed that the WJ-III $\mathrm{ACH}$ is measuring academic skills similar to those measured by other achievement tests: Wechsler Individual Achievement (reading comprehension $=0.79$; math reasoning $=0.60$ ); Kaufman Test of Educational Achievement (reading comprehension $=0.62$; math reasoning $=$ 0.29). Construct validity range of correlations for achievement clusters was $0.50-0.70$, indicating that the broad range of cognitive abilities are related to, but distinct from one another (Schrank, McGrew, \& Woodcock, 2001).

\section{Power and Sample Size}

Power analyses and sample size determination were conducted for the confirmatory factor analysis (CFA) and latent growth model (LGM). Analyses were conducted on two levels, parameter testing and data-model fit testing (Hancock, 2006). At the parameter testing level, sample size was determined ahead of time in order to conduct an $\alpha$-level statistical test of interest with a desired level of power. The necessary sample size was determined as: 


$$
n=1+\left[\frac{\lambda}{F_{M L}\left(\theta_{R}\right)}\right]
$$

where $\lambda$ is a noncentrality parameter and $F_{M L}\left(\theta_{R}\right)$ is a fit function. Thus, the sample size was determined if badness of fit of the reduced model is magnified. As this badness of fit increases, there is sufficient noncentrality to achieve the desired level of power for the $\alpha$-level test of the focal parameters. In other words, the test statistic is parameter-based such as a $\mathrm{X}^{2}$ difference test statistic that compares full and reduced models that have the focal parameters included or not. The 3-step process proposed by Hancock (2006) was followed with power and alpha levels set as follows: $1-\beta=0.80 ; \alpha=0.05$.

In order to evaluate the degree to data-model fit acceptability, Root Mean Square Error of Approximation (RMSEA) was employed as an assessment index. RMSEA represents a dispersal of data-model discrepancy across degrees of freedom and has often been used as a parsimonious data-model fit index. The null and alternative hypotheses are as follows: $H_{0}: R M S E A_{0} \geq 0.05$ and $H_{1}: R M S E A_{1}<0.05$ respectively. Table 2 presents the required sample size at each RMSEA level.

Table 2

Sample Size Requirement at Different RMSEA Levels

\begin{tabular}{cc}
\hline RMSEA & Sample size requirement \\
\hline .00 & $\approx 255$ \\
.02 & $\approx 330$ \\
.04 & $\approx 1455$ \\
\hline Note. $\lambda=27.56$ for the linear growth model in this study
\end{tabular}




\section{Analytic Plan}

The framework presented in Chapter 1 lends itself to an analytic approach that combines variable- and person-oriented approaches, as well as models that incorporate longitudinal analyses. Variable-oriented approaches assume that the population being studied is drawn from a homogenous group looking at relationships between variables. Person-oriented approaches to research assume that distinct subgroups possibly exist in the population and that aggregate-level analysis of variables may attenuate the relationship between the processes of interest, and thus affect the quality of the conclusions drawn by the study (von Eye \& Bergman, 2003; von Eye \& Bogat, 2006). Often, studies employ a variable-centered approach to predicting academic outcomes from maltreatment experiences and other individual or contextual factors (Muthén \& Muthén, 2000).

Though important in furthering our knowledge about academic outcomes of maltreated children, variable-oriented research can only provide an understanding of the relative contributions that predictor variables have on a specific outcome variable. Unfortunately, this variable to variable relationship may bring some limitations on our understanding of processes, particularly if these processes are concerned with children's development. If the questions asked are in line with developmental processes, growth, and individual-level differences, then personoriented analyses are more appropriate (Laursen \& Hoff, 2006; von Eye \& Bergman, 2003). In fact, Bergman and Vargha (2013) presented recommendations for matching specific methodologies for problems or inquiries from a developmental perspective, particularly around the use of person-oriented methods. The authors highlighted several ideas related to developmental science and how these ideas should match the selected methodology for research. For example, one highlighted idea was the view that individuals "should be viewed as a 
'functioning whole' and the focus is often shifted from variables as the main conceptual and analytical unit to the individual patterns of characteristics" (p. 10).

According to von Eye and Bergman (2003), the fundamental tenet of a person-oriented approach is that the holistic perspective of the individual is required to fully understand functioning and development. Laursen and Hoff (2006) added that person-oriented approaches describe differences among individuals in how variables relate to one another such that groups or types of individuals who share particular attributes are identified. Analytic methods employed as person-oriented models are appropriate for questions that examine group or individual differences in patterns of development and associations among variables (Laursen \& Hoff, 2006). Analytic techniques considered person-centered include latent profile, latent class, latent transition, and cluster analyses, as well as other finite mixture models.

The ultimate goal of this study then, was to identify these patterns of characteristics by identifying subgroups of children that differ in key growth parameters such as baseline and growth rate in an LGM framework or in latent classification for latent profile, class, and transition analyses. Additionally, this study aimed to examine how these classifications can be influenced by antecedents (i.e., time-invariant covariates) and consequences (i.e., distal outcomes). According to Petras and Masyn (2010) the complexity of the growth models and the different measurement model specifications for the latent classes must be taken into account. As such, the model building process must proceed in a systematic step-wise manner. All levels of analyses outlined here were conducted using SPSS v.21 and Mplus Student Version 7.3.

\section{Exploratory Data Analyses}

Preliminary analyses of the data were carried out in order to assess missing data patterns, prepare data for analyses, run univariate descriptive analyses, and examine correlations among 
the study variables. Sampling weights were employed to account for the complex sampling design. The NSCAW manual provided recommendations on how to incorporate complex sampling design when using SPSS in exploring descriptive statistics. As stated earlier, Mplus syntax accommodated complex sampling within its syntax. The level of variability and problematic observations such as outliers in the data were also examined.

\section{Model Specification and Identification}

According to Kline (2011), there are 6 steps to analysis using SEM. The first is to specify a model or models, followed by evaluation of model identification. The implication for underidentified or just-identified models includes not having sufficient observed variance or covariance information in order to estimate focal parameters. At the third step, measures were selected, and data were collected and screened. Model estimation occured at the fourth step. It is at this step that model fit was evaluated, parameter estimates interpreted, and consideration of equivalent models occurred. During step 5, the model is re-specified if model estimation does not yield tenable results. Finally, at step 6, results are reported.

This study focused on the trajectories of young maltreated children's core developmental processes of emotion regulation/dysregulation. Exploring this process, as well as answering the proposed research questions required a principled approach to model building. Subsequent models require that earlier models converge and that the models fit the data. Overall, these models have to be tenable and theoretically defensible. Additional specification and identification considerations specific to LGMs, GMMs, LCAs and LTA followed recommendations from Muthén (2004), Nagin and Odgers (2012), Nylund (2007) Petras and Masyn (2010), and Wang and Bodner (2007). 


\section{Latent Growth Models}

Latent growth models (LGM) using an SEM framework was employed to identify trajectories of emotion regulation. LGM provides information on an individual's initial levels as well as developmental trajectories to and from those levels, along with information on variability across individuals in both initial levels and trajectories. Additionally, LGMs provide a way to test contributions of other variables or constructs to explaining initial levels and trajectories (Hancock, Harring, \& Lawrence, 2013). According to Hancock, Kuo, and Lawrence (2001), LGM methods "simultaneously focus on correlation over time, changes in variance, and shifts in mean values...utilizing more information available in the measured variables than do traditional methods" (p. 471).

In a second-order latent growth model, first-order factors are modeled to be dependent on one or more second-order factors. Exogenous second-order factors only have first-order factors as indicators rather than manifest or measured variables. Second-order factors explain variance in, and covariance among, first-order factors (Hancock, 2001). Within the growth modeling context, repeated measures over time from the same individual are used to model first-order constructs, with latent growth parameters imposed as latent second-order factors. Similar to McArdle's (1988) “curve-of-factors model” and Tisak and Meredith's (1990) "latent variable longitudinal curve model," these exogenous second-order factors (i.e., latent intercepts and latent slopes) attempt to explain means, variances, and covariances of the first-order factors. That is, growth over time in the latent construct, rather than measured variables, is explained by these second-order latent intercept and slope factors.

When change is being assessed across $t$ time points, let $\eta_{j}$ be a latent construct that is indicated at time $j$ by $k$ measured variables $Y_{i j}(i=1, \ldots, k)$. This can be expressed in two steps, 
with the first equation representing a conventional first-order CFA (measurement) model with its mean structure modeled simultaneously:

$$
\mathbf{y}=\boldsymbol{\tau}+\Lambda \boldsymbol{\eta}+\boldsymbol{\varepsilon}
$$

Where vector $\mathbf{y}$ contains $t$ sets of values across time for $k, Y$ variables, $\boldsymbol{\tau}$ is a vector of variable intercepts, $\Lambda$ is a matrix of loadings relating measured variable indicators to their respective $\eta_{j}$ construct, and $\varepsilon$ is a vector of random normal errors. Modeling growth at the construct level can be described by:

$$
\boldsymbol{\eta}=\boldsymbol{\Gamma} \xi+\zeta
$$

Where $\boldsymbol{\Gamma}$ is a matrix of second-order factor loadings that reflect the hypothesized growth pattern underlying the $\eta_{t}$ constructs, $\xi$ is a vector of exogenous latent factors capturing the patterns for the growth being modeled, and $\zeta$ is a vector of random normal disturbances in the first-order $\eta_{t}$ constructs (Hancock, 2001; Hancock \& Buehl, 2008). See Figure 6 for a standard second-order latent growth model.

Use of LGM models is in line with Wulczyn et al.'s (2005) recommendation that wellbeing outcomes have an initial status and movement along a developmental trajectory. Within this analytic framework, intercept parameters can be interpreted as the initial or baseline status for individuals while the slope parameter can be interpreted as the rate of change across time. 


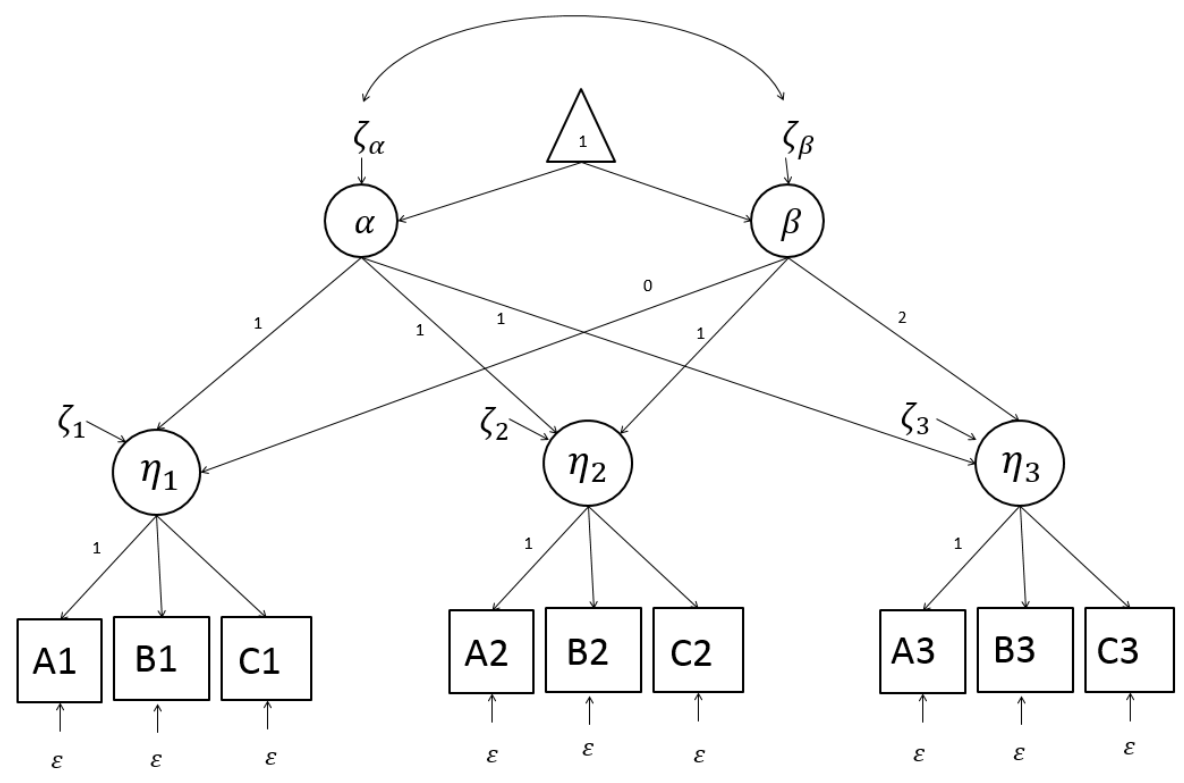

Figure 6. Standard structural diagram for a second-order latent growth model Adapted from Hancock and Buehl (2008). Circles represent first-order $(\eta)$ and second-order ( $\alpha ; \beta$ ) latent factors, and squares represent observed/manifest repeated measured variables taken at 3 time points.

\section{Mixture Models}

To accommodate person-oriented analyses, mixture models such as latent class analysis, latent transition analysis, and growth mixture models were incorporated in this study. Latent class analysis (LCA) is a class of mixture model to examine categorical indicators in order to model statistical parameters that differ across unobserved subgroups (Vermunt \& Magidson, 2002). Its longitudinal extension, latent transition analysis (LTA) examines transition probabilities for individuals switching from one latent class to another over time (Nylund, 2007).

Parameter estimates and individual class probabilities obtained from mixture models provide a description of group-level differences in amounts and patterns of within-person change and between-person differences. GMMs in particular, consider a separate growth model (and possibly growth function) for each of the latent classes that result from the model fitting process. The flexibility of mixture models can be seen in the inclusion of covariates and distal outcomes 
in modeling the resultant mixture classes. For example, a covariate can be included to predict latent class membership. Additionally, class membership can be related to a distal outcome, which provides predictive validity for the selected classes.

According to Wang and Bodner (2007), GMMs and other mixture models can be estimated using the maximum-likelihood approach. More specifically, an expectationmaximization (EM) algorithm is used. During the expectation (E) step, information on the latent class variable are considered missing and the conditional probability of individual $i$ belonging to the latent class $K$ (posterior probability of group membership) can be estimated. At the maximization (M) stage, the posterior probability for each individual is incorporated in the full data $\log$ likelihood function. This M step then maximizes the log likelihood function in the means and variance-covariance of the latent growth factors, factor loadings of longitudinal variables, logistic regression coefficients for covariates on the growth factors, and residual variances. Using FIML estimation method as the maximum likelihood approach at this stage allows for modeling of missing data as reported earlier, further adding to the flexibility of mixture models.

When employing mixture models, convergence on local rather than global maxima may arise, causing problems such as poorly behaved likelihood functions. Failure to consider the presence of local optima can have serious consequences such as selection of an inferior solution that differs from the actual maximum likelihood solution. According to Hipp and Bauer (2006), default values in software such as Mplus will have to be overridden in order to evaluate the parameter space and evaluate that the maximum likelihood solution has been obtained. One way to override the default values in current software is to use multiple start values from random locations in the parameter space, which can improve the chance of convergence to global 
maxima (Hipp \& Bauer, 2006; Petras and Masyn, 2010). Replication of the maximum likelihood value across multiple start values can increase the confidence that the solution obtained is a global maximum.

\section{Evaluation of data-model fit}

Once candidate models have been identified and specified, evaluation of data-model fit were conducted in order to ensure that the best fitting model was selected to answer each research question. Assessments of model fit were done on the confirmatory factor analyses models, latent growth curve models, latent class analyses models, and latent transition analyses models.

Confirmatory factor analysis and latent growth models. In order to evaluate datamodel fit quality for the CFA and LGM models presented above, model fit evaluations were conducted using indices proposed by $\mathrm{Hu}$ and Bentler (1999). These fit indices include absolute, parsimonious, and incremental index classes. From the absolute index class, the model $\mathrm{X}^{2}$ statistic (tested against $d f$ ) as well as the Standardized Root Mean Square Residual (SRMR $\leq .08)$ were assessed. From the parsimonious index class, evaluation of the Root Mean Square of Approximation (RMSEA $\leq .06)$ was done. Finally, from the incremental index class, Comparative Fit Index (CFI $\geq .95)$ were examined.

Mixture models. Additional data-model fit assessments specific to GMMs, LCAs, and LTAs were conducted. These assessments followed recommendations from Nylund (2007), Petras and Masyn (2010), and Wang and Bodner (2007). Nested model tests and fit indices are important assessment tools to evaluate model fit when estimating number of classes, deciding on the smallest appropriate number of classes, and overall model fit. In addition to these statistical 
criteria, the value and utility of the resultant classes were assessed, as well as qualitative evaluations of face validity and usefulness of latent class extractions.

Model fit comparisons were based on log likelihood values rather than standard chisquare difference tests. These log likelihood tests included the Vuong-Lo-Mendell-Rubin test (VLMR-LRT) and the parametric bootstrapped Likelihood Ratio Test (BLRT). The VLMR-LRT approximates the LRT distribution when comparing a $k$-class to a $(k$-g)-class finite mixture model where classes differ in the mean structure (Petras \& Masyn, 2010). BLRT uses bootstrap samples to empirically derive the sampling distribution of the LRT statistic. These tests compare a $(k-1)$-class null model with a $k$-class alternative (less restrictive) model. A statistically significant $p$-value suggests that the $k$-class model fits the data significantly better than a model with $k$-1 fewer classes (Petras \& Masyn, 2010; Wang \& Bodner, 2007).

Likelihood-based information indices such as the Bayesian Information Criteria (BIC) and sample-size adjusted BIC were used for model selection in addition to the nested model fit procedures highlighted above (Petras \& Masyn, 2010; Wang \& Bodner, 2007). These indices are calculated as a function of the log likelihood with a penalty for model complexity. Model complexity is defined as the number of parameters estimated relative to the sample size. Lower values on BIC and adjusted BIC generally indicated a better model. Nylund, Asparouhov, and Muthén (2007) recommended the use of BIC and VLMR-LRT for the initial model selection, then inclusion of the BLRT to compare a smaller set of models.

The value and utility of the resulting classes were examined in addition to the statistical diagnostics presented above. Specifically, the use entropy was recommended by Petras and Masyn (2010). According to the authors, "entropy summarizes the degree to which the latent classes are distinguishable and the precision with which individuals can be placed into classes" 
(p.80). Entropy is a measure of class separation with the purpose of finding homogenous groupings of individuals with distinct trajectories wherein the between-class variability is much greater than the within-class dispersion, resulting in orthogonal separation of classes. Entropy is also a function of the individual estimated posterior probabilities. Entropy values range from 0 to 1 where higher values indicate better class separation.

Additional qualitative evaluations of the usefulness and face validity of the latent classes were done in order to corroborate the quantitative analyses specified above. Additional assessment can be accomplished by examining and interpreting the estimates and plots of the model-implied mean class trajectories for different models. Examining class size and proportions will be important to note since over-extraction of classes might be present. Finally, the use of auxiliary information such as antecedent (i.e., language, gender, caregiver emotional support, and cognitive stimulation) and consequent (academic achievement) variables, informed by theory, were examined to assess the concurrent validity of the candidate models.

These evaluations of data-model fit procedures were first conducted on unconditional mixture models prior to the inclusion of covariates and distal outcomes. This is especially important in order to prevent the selection of a model with misspecifications that have not been addressed. Doing so may result in the improper and inaccurate interpretation of the model parameters that have serious implications for interpretation of the results (Wang \& Bodner, 2007).

\section{Analysis of Research Questions}

Measurement models were first specified prior to answering the stated research questions. In so doing, factor structures for emotion regulation/dysregulation can be examined. This ensures that the construct of interest in subsequent growth models were indeed of emotion 
regulation/dysregulation. Once measurement models were specified, the next step entailed analysis of the first research question using LGMs. This was then followed by procedures to answer research questions 2,3 , and 4 .

Measurement Models. Prior to exploring emotion regulation development using the latent growth models and subsequent exploration of mixture models, covariates, and distal outcome, measurement models were specified to ensure validity of the factor structure for emotion regulation/dysregulation. These measurement model specifications were conducted at each wave of data collection (i.e., cross-sectional) and longitudinally. Item and construct reliability were assessed for the cross-sectional and longitudinal models.

Factorial invariance. Longitudinal measurement invariance is required in any secondorder latent growth modeling framework (Ferrer, Balluerka, \& Widaman, 2008; Harring et al., 2012). An implicit assumption related to this requirement is that growth in the first-order latent level reflects true changes in the underlying theoretical phenomena of interest. This is in contrast to changes related to non-invariant measurement models or a shift in observed variable indicators that do not measure the same construct at different age groups (Ferrer et al., 2008; Hancock \& Buehl, 2008; Harring et al., 2012). To ensure measurement invariance, constraints were placed on CBCL and SSRS items across the 3 waves of data collection.

Research Question 1. What are the growth trajectory patterns of emotion regulation development for young maltreated children across 3 years since time of first contact with Child Protective Services?

The aim of this first question was to examine growth curves and determine the functional form for emotion regulation development across 3 time points since first contact with CPS during the preschool period. Information on an individual's initial levels and developmental 
trajectories to and from those levels on measures of emotion regulation were of interest. To answer this question, first-order and second-order latent growth models using both ordinal assumptions and factor-saved scores were specified to examine growth trajectories on the emotion dysregulation latent construct derived from the earlier measurement models. Invariance constraints were placed on the indicator items, particularly in the ordinal analysis. Invariance constraints ensured that the thresholds for each item responses were the same across time and ensures that changes in response probabilities were a reflection of changes in the construct rather than changes in item functioning.

Research Question 2. Is there heterogeneity in young maltreated children's display of emotion regulation and do they exhibit stability and change in these developmental patterns across time?

Lack of significant growth parameters in the LGM models prompted the use of Latent class analysis (LCA) and its longitudinal extension, latent transition analysis (LTA) in answering this question. LCA and LTA models were employed to examine possible emotion regulation/dysregulation classes in which maltreated children belong, as well as to examine how children's regulatory classes might change across 3 years since initial contact with CPS. Ultimately, this question aims to enumerate the number of latent classes in the sample of maltreated preschool children.

Research Question 3. To what extent do emotion regulation class memberships differ by gender, language, placement stability, caregiver emotional support, and caregiver cognitive stimulation?

Based on discussions from Chapters 1 and 2, developmental theories state that different subgroups of people follow different developmental trajectories across their lifespan. 
Specifically, bio-ecological theory (Bronfenbrenner \& Morris, 2006) attributes change in developmental outcomes as a result of proximal processes between the child and his or her environment. If these environmental influences hold, then hypothetical variations in developmental trajectories should be present. These hypothetical latent classes of individuals are distinguishable by different developmental patterns across time. Research question 3 was answered by incorporating gender, language, placement stability, caregiver emotional support, and caregiver cognitive stimulation in a multinomial logistic regression framework as predictors of emotion regulation/dysregulation class membership.

Research Question 4. What are the different pathways in which emotion regulation predicts later academic achievement for young maltreated children who had contact with CPS during the preschool period?

The aim of this question was to understand the multiple pathways to academic achievement for young maltreated children. Understanding the impact that placement stability and changing caregiver quality on changes in school readiness domains is important. By uncovering how these predictors influence class membership and thus, later academic achievement, policies and interventions can focus on these predictors as mechanisms of change to help close the achievement gap for these understudied and vulnerable children. In order to answer this research question, a second-order LTA model was used to examine changes in emotion regulation/dysregulation class membership across time. Subtests of the WJ-III that assessed math and reading achievement were then included as a distal outcome at wave 4 . Thus, answers to this question can provide information on the stability of emotion regulation/dysregulation patterns and how classification of maltreated children at 3 years after CPS investigation might impact school achievement at 53-72 months post-investigation. 


\section{Chapter 4: Results}

This study had four goals for understanding the core developmental process of young maltreated children. The first was to determine the growth patterns of emotion dysregulation (EdR) across three waves of data collection that were 18 months apart, beginning with preschool age at baseline. The second goal was to determine whether there are unobserved patterns (i.e., heterogeneity of subgroups of maltreated children) and to determine the number of these developmental trajectory patterns present in the current sample. The third goal was to examine how gender, language, placement instability, caregiver emotional support, and caregiver cognitive stimulation impact development by predicting membership in specific latent classes. Finally, this study examined how particular classes represented developmental pathways that predicted later academic achievement.

To achieve these goals, this study employed longitudinal analyses and person-centered approaches that followed a cohort of children entering the child welfare system during the preschool period. As stated in Chapter 2, preschool-aged children are a vulnerable and understudied population. Longitudinal methods to study changes over time can provide information on how development proceeds. Additionally, person-centered methods using finite mixture models can provide information on unobserved subgroups of children that can provide information on different pathways of development.

The majority of the studies reviewed in Chapter 2 only examined the academic outcomes for long-term foster children. Because not every child investigated by CPS is placed in long term foster care, there remains a gap in understanding early developmental processes for children that remain in their homes or are temporarily removed. Thus, the sample for this study was drawn from the NSCAW CPS sample rather than the LTFC sample. 


\section{Analytic Sample for the Study}

The NSCAW data were divided by waves into separate files, making longitudinal analysis of repeated measures challenging. The first step in preparing the data for analysis entailed merging these files into a single analytic dataset. According to the NSCAW Data File User's Manual (DFUM; 2008), this process of "stacking” the data is essential prior to any longitudinal analyses and the manual provided recommendations for these procedures. The DFUM recommended the use of the "NSCAWID" variable as the merge variable for combining data from multiple waves into a single data record. The NSCAWID was a unique child identifier that allowed each child-level data from multiple respondents and related variables to be included during the merge process. The next step was to identify the different age groups that make up the CPS sample. Table 3 lists the distribution of ages for the 5,501 children in the CPS sample. Data on participant children in the study included information from 4 sources: the child, current caregiver, CPS caseworker/investigator, and a teacher. In order to confirm the age for the missing case, a visual inspection of the data was conducted and child age was compared across the 4 informants. The final age in the 3-5 range was then added when age information was confirmed across informants.

Table 3

Distribution of Age Groups in the Total NSCAW CPS Sample

\begin{tabular}{lcc}
\hline & $n$ & $\%$ \\
\hline Missing & 0 & .0 \\
0-2 years & 1996 & 36.3 \\
3-5 years & 834 & 15.2 \\
6-10 years & 1492 & 27.1 \\
11+ years & 1179 & 21.4 \\
\hline Total & 5501 & 100.0 \\
\hline
\end{tabular}


The preschool subsample was then extracted from the full data, yielding a final sample of 834 preschool-aged (i.e., 3-5 years old) children from baseline. This was the final sample for subsequent analyses across baseline (i.e., wave 1), wave 3, wave 4, and wave 5. Of the 834 preschool-aged children at baseline, 3-year olds comprised of 35\% $(n=291)$ of the sample; fourand five-year olds made up 34\% $(n=287)$ and $31 \%(n=256)$, respectively. The mean and standard deviation of the children's ages at subsequent waves are as follows: wave $3(M=5.25$ years old, $S D=.94)$, wave $4(M=6.56$ years old, $S D=.95)$, and wave $5(M=10.21$ years old, $S D=1.45)$. Table 4 presents a summary of the children's demographic information. Gender was almost evenly split between boys and girls, with the sample being predominantly White children. Similar to what is typically found in the literature, neglect was the predominant maltreatment type experienced by the children in this sample.

Table 4

\section{Child Demographics Information}

\begin{tabular}{lcc}
\hline Characteristic & \multicolumn{2}{c}{ Wave 1 } \\
& $n$ & $\%$ \\
\cline { 2 - 3 } & & \\
\cline { 2 - 3 } Gender & 438 & 52.5 \\
Boys & 396 & 47.5 \\
Girls & & \\
Race & 45 & 5.4 \\
Native American & 17 & 2 \\
Asian/Pacific Islander & 246 & 29.5 \\
Black/non-Hispanic & 471 & 56.5 \\
White/non-Hispanic & 55 & 6.6 \\
Other & & \\
Hispanic & 152 & 18.2 \\
Yes & 681 & 81.7 \\
$\quad$ No & 1 & 0.1 \\
Refused & & \\
Type of maltreatment & 191 & 22.9 \\
Physical abuse & 107 & 12.8 \\
Sexual abuse & 43 & 5.2 \\
Emotional abuse & &
\end{tabular}




\begin{tabular}{lcc}
\hline Characteristic & \multicolumn{2}{c}{$\begin{array}{c}\text { Wave } 1 \\
(n=834)\end{array}$} \\
\cline { 2 - 3 } & $n$ & $\%$ \\
\hline Neglect & 372 & 44.6 \\
Other & 55 & 6.59 \\
Missing & 66 & 7.91 \\
\hline
\end{tabular}

Note. 3-5 years old at wave 1

Information on alleged abuse type was derived from the caseworker/investigator instrument and was available only at baseline. Subsequent waves included information on new investigations or allegations only. For the purpose of this study, only the original nature of abuse information that resulted in Child Protective Services (CPS) contact was included in descriptive analyses. The NSCAW data were selected for this study because every child included in the survey had been referred to CPS. Because this study's main goal was to understand the developmental processes of young children with a history of overall maltreatment, maltreatment subtype analyses were not conducted.

Child placement information is presented in Table 5. A majority of the children remained in their homes during the baseline interviews. The remainder of the children lived in out-of-home $(\mathrm{OOH})$ placements mostly with relatives or with non-relative foster homes. A majority of the children $(99.3 \% ; n=828)$ lived with the caregiver who completed baseline interviews. A majority of the children in this sample were not removed from their homes, whereas those who were removed only experienced a single occurrence. Less than $1 \%$ of the sample experienced $2-4$ removals from their homes. 
Table 5

Child Placement Information

\begin{tabular}{lcccccc}
\hline \multirow{2}{*}{ Variable } & \multicolumn{2}{c}{ Wave 1} & \multicolumn{2}{c}{ Wave 3} & \multicolumn{2}{c}{ Wave 4} \\
\cline { 2 - 7 } & $n$ & $\%$ & $n$ & $\%$ & $n$ & $\%$ \\
\hline Child ever removed & 98 & 11.8 & - & - & - & - \\
$\quad$ Yes & 735 & 88.1 & - & - & - & - \\
No & 1 & 0.1 & - & - & - & - \\
$\quad$ Refused & & & & & & \\
Number of times removed & 91 & 10.9 & - & - & - & - \\
1 & 4 & 0.5 & - & - & - & - \\
2 & 2 & 0.2 & - & - & - & - \\
3 & 1 & 0.1 & - & - & - & - \\
4 & 736 & 88.2 & - & - & - & - \\
Not applicable & & & & & & \\
Home setting & 630 & 75.5 & 509 & 61 & 510 & 61.2 \\
In-home: bio parent & 6 & 0.7 & 13 & 1.6 & 33 & 4 \\
In-home: adoptive parent & 42 & 5 & 56 & 6.7 & 76 & 9.1 \\
In-home: other & 151 & 18.1 & 124 & 14.9 & 84 & 10.1 \\
Out-of-home & 5 & 0.6 & 132 & 15.8 & 131 & 15.7 \\
Missing & & & & & & \\
Out-of-home placement & 72 & 8.6 & 67 & 8 & 45 & 5.4 \\
Foster home & 77 & 9.2 & 64 & 7.7 & 33 & 4 \\
Kinship care & 3 & 0.4 & 1 & 0.1 & 2 & 0.2 \\
Group home & 4 & 0.5 & 3 & 0.4 & 2 & 0.2 \\
Other & 678 & 81.3 & 594 & 71.2 & 632 & 75.8 \\
Not applicable & - & - & 105 & 12.6 & 120 & 14.4 \\
Missing & & & & & & \\
\hline
\end{tabular}

School placement at baseline indicated that $39 \%(n=329)$ of the children were not enrolled in formal schooling; 37\% $(n=308)$ were enrolled in nursery, preschool, or Head Start. Additionally, 21\% $(n=174)$ of the children were in Kindergarten, while $1 \%(n=8)$ were in ungraded placement or informal care. There were 14 children reported to be in first grade and 1 child reported to be in the fourth grade, which appear to be a data entry error. School placement information across waves 1,3 , and 4 are presented in Table 6 . Wave 5 grade placement for this cohort of children are presented in Table 7. 
Table 6

School Placement Information

\begin{tabular}{|c|c|c|c|c|c|c|}
\hline & \multicolumn{2}{|c|}{ Wave 1} & \multicolumn{2}{|c|}{ Wave 3} & \multicolumn{2}{|c|}{ Wave 4} \\
\hline & $n$ & $\%$ & $n$ & $\%$ & $n$ & $\%$ \\
\hline $\begin{array}{l}\text { Nursery/Preschool/Head } \\
\text { Start }\end{array}$ & 308 & 36.9 & 170 & 20.4 & 14 & 1.7 \\
\hline Kindergarten & 174 & 20.9 & 241 & 28.9 & 136 & 16.3 \\
\hline $1^{\text {st }}$ Grade & 14 & 1.7 & 164 & 19.7 & 279 & 33.5 \\
\hline $2^{\text {nd }}$ Grade & - & - & 29 & 3.5 & 196 & 23.5 \\
\hline $3^{\text {rd }}$ Grade & - & - & - & - & 57 & 6.8 \\
\hline $4^{\text {th }}$ Grade & 1 & .1 & - & - & 1 & .1 \\
\hline $5^{\text {th }}$ Grade & - & - & 1 & .1 & 2 & .2 \\
\hline Ungraded placement & 8 & 1 & 3 & .4 & 4 & .5 \\
\hline Child not in school & 329 & 39.4 & 90 & 10.8 & 10 & 1.2 \\
\hline Don’t know & - & - & 1 & .1 & - & - \\
\hline Non-interview & - & - & 135 & 16.2 & 133 & 15.9 \\
\hline Legitimate skip & - & - & - & - & 2 & .2 \\
\hline Total & 834 & 100 & 834 & 100 & 834 & 100 \\
\hline
\end{tabular}

Table 7

Grade Level at Wave 5

\begin{tabular}{lcc}
\hline & $n$ & $\%$ \\
\hline Legitimate skip & 4 & .5 \\
Non interview & 208 & 24.9 \\
$1^{\text {st }}$ Grade & 5 & .6 \\
$2^{\text {nd }}$ Grade & 69 & 8.3 \\
$3^{\text {rd }}$ Grade & 137 & 16.4 \\
$4^{\text {th }}$ Grade & 59 & 7.1 \\
$5^{\text {th }}$ Grade & 138 & 16.5 \\
$6^{\text {th }}$ Grade & 143 & 17.1 \\
$7^{\text {th }}$ Grade & 62 & 7.4 \\
$8^{\text {th }}$ Grade & 8 & 1.0 \\
$9^{\text {th }}$ Grade & 1 & .1 \\
\hline Total & 834 & 100 \\
\hline
\end{tabular}

Demographic information for the children's caregivers who responded at baseline are presented in Table 8. A majority of the caregiver respondents were the children's biological parent under the age of 35. Most had at least a high school education and worked at least part- 
time. Information from the Health and Human Services poverty guidelines (see Appendix C) was used to derive poverty levels. Further, information from the income module (IN) was used along with household size to estimate poverty levels for the current sample. Given that income information from NSCAW was presented in categorical format rather than continuous, and that this variable was not central to the study, poverty status was only approximated and not used in the final analysis. A cross-tabulation procedure was conducted to examine where income levels and household size coincided with the particular HHS poverty guidelines to consider whether or not a family can be classified as living in poverty (see Appendix D). Based on HHS guidelines, the following proportions of families were classified as living in poverty: $386(46.3 \%)$ at Wave $1,334(40.1 \%)$ at Wave 3, and $329(39.5 \%)$ at Wave 4.

Table 8

Caregiver Demographics at Wave 1

\begin{tabular}{lcc}
\hline \multicolumn{1}{c}{ Characteristic } & $n$ & $\%$ \\
\hline Participants & 834 & - \\
Age & & \\
$\quad$ <35 years & 558 & 66.9 \\
35-44 years & 164 & 19.7 \\
45-54 years & 75 & 9 \\
>54 years & 36 & 4.3 \\
$\quad$ Refused & 1 & 0.1 \\
Gender & & \\
$\quad$ Male & 85 & 10.2 \\
Female & 749 & 89.8 \\
Race & & \\
$\quad$ Native American & 41 & 4.92 \\
Asian/Pacific Islander & 17 & 2.04 \\
Black/non-Hispanic & 200 & 23.98 \\
White/non-Hispanic & 443 & 53.12 \\
Hispanic & 121 & 14.51 \\
Other & 10 & 0.12 \\
Refused & 2 & 0.24 \\
Child's biological parent & & \\
$\quad$ Yes & 569 & 68.2 \\
No & 265 & 31.8
\end{tabular}




\begin{tabular}{|c|c|c|}
\hline Characteristic & $n$ & $\%$ \\
\hline \multicolumn{3}{|l|}{ Marital status } \\
\hline Married & 306 & 36.7 \\
\hline Separated & 113 & 13.5 \\
\hline Divorced & 146 & 17.5 \\
\hline Widowed & 21 & 2.5 \\
\hline Never married & 247 & 29.6 \\
\hline Refused & 1 & 0.1 \\
\hline \multicolumn{3}{|l|}{ Employment status } \\
\hline Full-time & 323 & 38.7 \\
\hline Part-time & 113 & 13.5 \\
\hline Unemployed & 113 & 13.5 \\
\hline Does not work & 260 & 31.2 \\
\hline Other & 24 & 2.9 \\
\hline Does not know & 1 & 0.1 \\
\hline \multicolumn{3}{|l|}{ Highest education level } \\
\hline Beyond high school & 206 & 24.7 \\
\hline High school & 412 & 49.4 \\
\hline Less than high school & 214 & 25.7 \\
\hline Ungraded place & 1 & 0.1 \\
\hline Refused & 1 & 0.1 \\
\hline \multicolumn{3}{|c|}{ Total family income per year } \\
\hline$<\$ 5000$ & 58 & 7 \\
\hline$\$ 5000-\$ 9999$ & 133 & 15.9 \\
\hline$\$ 10000-\$ 14000$ & 121 & 14.5 \\
\hline$\$ 15000-\$ 19999$ & 85 & 10.2 \\
\hline$\$ 20000-\$ 24999$ & 89 & 10.7 \\
\hline$\$ 25000-\$ 29999$ & 60 & 7.2 \\
\hline$\$ 30000-\$ 34999$ & 41 & 4.9 \\
\hline$\$ 35000-\$ 39999$ & 44 & 5.3 \\
\hline$\$ 40000-\$ 44999$ & 24 & 2.9 \\
\hline$\$ 45000-\$ 49999$ & 22 & 2.6 \\
\hline$>\$ 50000$ & 83 & 10 \\
\hline Partial interview & 3 & 3.8 \\
\hline Refused & 39 & 4.7 \\
\hline Don't know & 32 & 0.4 \\
\hline
\end{tabular}

\section{Missing Data}

According to the NSCAW data file manual (DFUM, 2008), prior analyses have been conducted with the NSCAW data to ensure that the data were missing at random. Additionally, the use of sampling weights ensured that the nonresponse and non-coverage bias due to the 
complex sampling design was accounted for. Missing data analysis was conducted using Mplus Student version 7.1. Frequencies of missing data patterns can be found in Appendix E. Based on these results, the most frequently occurring pattern did not have missing values on all endogenous and exogenous variables. A majority of the frequency patterns included missing values on the exogenous variables (i.e., CBCL, SSRS, and Woodcock Johnson-III). Some of the data patterns had missing values on the endogenous variables, though frequency counts were negligible. Given that subsequent analyses employed Maximum Likelihood (ML) and Full Information Maximum Likelihood (FIML), which was able to estimate missing values on the exogenous variables, missing data was not a problem and no further imputation methods were conducted.

\section{Data Cleaning}

To address the problem of missing data with the CBCL and SSRS due to instrument changes based on age of administration, several steps were conducted to ensure that all items or indicators were present across waves 1, 3, and 4. For both the CBCL and SSRS, overlapping items were first selected from each of the attention, aggression, anxiety/depression, and selfcontrol subscales (see Appendix F for tables that outline the overlapping items). Because items were ordered differently based on the instrument version (i.e., BC for 2-3 year olds and TC for 418 year olds), items were reordered and relabeled to maintain consistency. The final 21 items used in the study are included in Appendix G. The Likert-type responses for these items were $0=$ not true, 1 = somewhat or sometimes true, 2 = very often or often true. This order was retained from the original response categories where higher scores indicate increased problem behaviors.

Similar steps to the CBCL item reduction procedures were conducted on the self-control subscale of the SSRS. The final items retained for the study are presented in Appendix H. These 
included the overlapping items from the preschool (PS) and the elementary (PT) versions of the instrument. The original Likert-type responses ranged from $0=$ never, $1=$ sometimes, and $2=$ very often, which indicated that a child exhibited self-control. In order to be parallel with the emotion dysregulation construct purportedly measured by the CBCL items, the SSRS was reverse coded. The recoded SSRS response items were changed to $0=$ very often, $1=$ sometimes, and $2=$ never where higher scores indicated problems with self-control. Item texts were also altered in order to reflect the reversed direction of the current items employed in this study.

Creation of the placement stability covariate was supposed to replicate procedures from Casanueva et al. (2014). However, difficulties were encountered when some of the stated variables were not available in the current dataset. For example, the caseworker question that asked, "where is the child currently living?" was not located in the caseworker instrument. Another source of problem was the question that asked about the number of times that the respondent and child moved together. According to Casanueva et al. (2014), a change in caregiver was counted if a child was placed in a new household for more than 7 days and the original caregiver did not move with the child. This question (item PLE20A) was present at the baseline interview but not in subsequent waves. In fact, the Data File User's Manual (DFUM) stated that questions in the PLE modules were only administered at Wave 1.

In order to address this problem, an alternate procedure was conducted. Using the NSCAW study-derived variables that categorized the child's placement setting at each wave (i.e., CHDHMSET, CH3HMSET, and CH4HMSET), a score of 0 or 1 was assigned if changes in placement occurred. For example, if placement setting was equal across all time points, then a score of 0 was given due to stability in placement across all waves. If changes in placement 
occurred at any point in time, then a score of 1 was assigned. See Table 9 for the final count in placement instability.

Table 9

Frequency of Placement Instability

\begin{tabular}{|c|c|c|}
\hline Instability score & $n$ & $\%$ \\
\hline 0 (stable) & 465 & 55.8 \\
\hline 1 (change in placement) & 293 & 35.1 \\
\hline Missing & 76 & 9.1 \\
\hline TOTAL & 834 & 100 \\
\hline
\end{tabular}

\section{Descriptives}

Descriptive statistics for the study's covariates, along with the distal outcome of academic achievement are presented in Table 10. Similar to the CBCL and SSRS preliminary analyses, problems with shifting measures for the cognitive stimulation, emotional support, and language instruments were detected. Procedures similar to the CBCL and SSRS reordering and recoding could not be performed, however, on these covariate measures due to difficulty with the data structure. As such, only wave 1 measures for the HOME inventory (cognitive stimulation and emotional support) and the PLS (language) were used. The distal outcome of academic achievement was measured using the 4 standardized scores on the subscales of the WoodcockJohnson III at wave 5. These subscales measured math (applied problems and calculation) and reading (letter-word identification and passage comprehension) achievement in early elementary. 
Table 10

Descriptive Statistics for Time-invariant Covariates from Wave 1 and Distal Outcome at Wave 5

\begin{tabular}{|c|c|c|c|c|c|c|}
\hline Variable & $n$ & $M$ & $S D$ & Range & Skewness & Kurtosis \\
\hline Cognitive stimulation & 832 & 10.69 & 2.291 & $0-14$ & -1.025 & 1.913 \\
\hline Emotional support & 832 & 8.53 & 2.30 & $0-12$ & -0.997 & 0.785 \\
\hline Language & 751 & 86.43 & 20.57 & $50-135$ & -0.008 & -0.760 \\
\hline \multicolumn{7}{|l|}{ Academic achievement } \\
\hline Applied problems & 598 & 95.18 & 16.56 & $1-129$ & -2.222 & 10.135 \\
\hline Calculation & 598 & 95.68 & 18.55 & $9-176$ & -1.469 & 5.296 \\
\hline $\begin{array}{l}\text { Letter-word } \\
\text { identification }\end{array}$ & 598 & 97.48 & 18.62 & $1-157$ & -1.164 & 4.604 \\
\hline $\begin{array}{l}\text { Passage } \\
\text { comprehension }\end{array}$ & 598 & 89.05 & 15.37 & $1-117$ & -2.411 & 10.176 \\
\hline
\end{tabular}

Results indicated a problem with skewness and kurtosis on all the covariates except for language. This means that univariate normality assumptions may have been violated. The next step was to analyze outliers to examine its potential impact on the data distribution. Boxplots with the outlier cases are presented in Appendix I. There appeared to be extreme values identified by the cases on the lower and upper bound sections of the plots. Descriptive results were further examined. Mean values for each measure were compared with the $5 \%$ trimmed mean to evaluate the magnitude of the discrepancy between the value that included the outlier cases, with those that were excluded. Table 11 displays the trimmed mean along with the confidence intervals. Based on these results, it appears that the outliers did not contribute significantly to the skewed distribution of the covariates and distal outcome variables. Given that the study employed a sample of maltreated children, it may be possible that the lower score values were truly indicative of the participants' scores on these measures. Indeed, based on the studies reviewed in Chapter 2, children with a history of maltreatment often score below their peers in standardized achievement tests and language measures compared to non-maltreated peers. Skewed results from the caregiving measures could also reflect underlying values that are 
expected with this population. Referrals to CPS are often made due to compromised caregiving. Given that a majority of the sample were the caregivers who were the subject of the investigation, lower scores on the HOME may reflect lower quality of caregiving. Given that the mean values fell within the CI limits and that differences with or without the outliers were negligible, a decision to proceed with the full sample was made.

Table 11

Comparison of Mean Values with Outliers Excluded

\begin{tabular}{|c|c|c|c|c|c|}
\hline \multirow{3}{*}{ Variable } & \multirow{3}{*}{$n$} & \multirow{3}{*}{$M$} & \multirow{3}{*}{$5 \%$ Trimmed $M$} & \multicolumn{2}{|c|}{$95 \%$ CI for the $M$} \\
\hline & & & & Lower & Upper \\
\hline & & & & Bound & Bound \\
\hline Cognitive stimulation & 832 & 10.69 & 10.82 & 10.53 & 10.84 \\
\hline Emotional support & 832 & 8.53 & 8.68 & 8.38 & 8.69 \\
\hline Language & 751 & 86.43 & 86.32 & 84.96 & 87.90 \\
\hline \multicolumn{6}{|l|}{ Academic achievement } \\
\hline Applied problems & 598 & 95.18 & 96.44 & 93.85 & 96.51 \\
\hline Calculation & 598 & 95.68 & 97.03 & 94.19 & 97.17 \\
\hline $\begin{array}{l}\text { Letter-word } \\
\text { identification }\end{array}$ & 598 & 97.48 & 98.38 & 95.99 & 98.98 \\
\hline $\begin{array}{l}\text { Passage } \\
\text { comprehension }\end{array}$ & 598 & 89.05 & 90.38 & 87.82 & 90.29 \\
\hline
\end{tabular}

\section{Correlations}

Once data cleaning and diagnostics were conducted, bivariate correlation analyses were done across all endogenous and exogenous variables. Results are presented in Appendix J. Looking among the covariates, there did not appear to be any significant relationship between gender and maltreatment type. Being female was positively related to language and the reading subscales of the Woodcock Johnson-III (i.e., letter-word identification, passage comprehension). Gender had no correlation with the math subscales (i.e., calculation and applied problems). Maltreatment type was significantly related to language, though no relationship with academic achievement. Placement instability was related to type of maltreatment but had no significant 
relationship with the other covariates and academic achievement. Instability was only related to a few of the CBCL and SSRS items.

Examining the individual items for the CBCL and SSRS, acting young for age was related to all the covariates, as well as with academic achievement. Most of the attention problem items (e.g., can’t pay attention, can’t sit still) were related to academic achievement and language. Being male had significant correlations with the physical aggression items (e.g., destroys things, disobedient at home, does not respond appropriately when hit by other children). Academic achievement measures were also related to the items that asked about physical aggression. A majority of the CBCL and SSRS items were correlated with each other within each wave (i.e., cross-sectional), as well as across all the waves (i.e., longitudinal).

\section{Measurement Models for Emotion Dysregulation}

Specification of measurement models that represent emotion dysregulation was needed prior to the growth trajectory and subgroup analyses. Doing so ensured that misspecifications of these models did not enter into the structural model during the examination of emotion dysregulation for the 834 preschool-aged children who had prior contact with Child Protective Services (CPS).

Based on the studies reviewed in Chapter 2, along with the theory of change proposed by the bio-ecological model, a single factor model was specified wherein items on the Child Behavior Checklist (CBCL) and the Social Skills Rating System (SSRS) were loaded onto the emotion dysregulation (EdR) latent factor. Specifically, the CBCL subscale items of anxiety/depression, aggression, and attention (items A1-A21), along with the SSRS subscale items for self-control (items B1-B6), were selected as ordinal indicators that represented EdR. Scores ranged from 0 to 2, with higher scores indicating higher levels of emotion dysregulation. 
As discussed in Chapter 3, items on the self-control subscale of the SSRS were reverse-coded to ensure that the direction of the response items were similar to the CBCL.

A series of cross-sectional confirmatory factor analyses was conducted to examine the loading structures of the EdR items for waves 1,3, and 4. This was followed by a longitudinal confirmatory factor analysis (CFA) to assess the loading structures of the EdR items simultaneously across the three data collection waves. Raw data were analyzed using Mplus version 7.3 (Muthén \& Muthén, 2012), which included stratification, cluster, and weight variables to account for the complex sampling design. A mean- and variance-adjusted weighted least squares (WLSMV) estimation method was selected based on the ordinal nature of the indicators. Data-model goodness of fit was evaluated using absolute (i.e., Model $\mathrm{X}^{2}$ ), parsimonious (i.e., RMSEA), and incremental (i.e., CFI) fit indices. Recommendations proposed by $\mathrm{Hu}$ and Bentler (1999) will be followed where acceptable data-model fit should meet the following criteria as stated in Chapter 3: Model $X^{2}(p>0.001)$, Root Mean Square of Approximation (RMSEA $\leq 0.06,90 \% \mathrm{CI} \leq 0.06)$, and Comparative Fit Index (CFI $\geq 0.95)$. The Standardized Root Mean Square Residual (SRMR) values were not computed due to the ordinal nature of the EdR indicator items. For categorical variables using the WLSMV estimator, Mplus computes the Weighted Root Mean Square Residual (WRMR) instead.

The WRMR fit index employs a variance-weighted method for models with categorical indicators (Cook, Kallen, \& Antnabb, 2009). According to Muthén (2010), WRMR is an experimental fit statistic that requires further examination. However, in a study by $\mathrm{Yu}(2002)$, WRMR cut-off values of 0.95 or 1.0 has "acceptable Type I error rates with small to moderate Type II error rates in the CFA and MIMIC models...” (p. 161). Given the limited empirical evaluation of the WRMR fit index, a decision was made to exclude it as a fit index. 
Construct reliability is important in assessing the the quality of the resulting measurement model. According to Gagne and Hancock (2006), the quality of the measurement model should take into account the magnitude of the factor loadings and the number of indicator variables selected for the construct. Cronbach's $\alpha$ and coefficient $H$ were selected as measures of internal consistency and construct reliability for this study. Coefficient $H$ or maximal reliability can be expressed for a $k$-indicator factor using the standardized loadings $\left(a_{i}\right)$ :

$$
H=\frac{\sum_{i=1}^{k} \frac{a_{i}^{2}}{\left(1-a_{i}^{2}\right)}}{1+\sum_{i=1}^{k} \frac{a_{i}^{2}}{\left(1-a_{i}^{2}\right)}}
$$

According to the authors, coefficient $H$ has several advantages. First, reliability does not decrease with additional indicators. Second, reliability is not affected by the sign of the loadings. This is important given that the items for self-control loaded negatively on emotion dysregulation. Finally, coefficient $H$ cannot be smaller than the reliability of the best indicator..

\section{Cross-sectional CFA}

The CFA models at waves 1, 3, and 4 were over-identified with $324 d f$ and 81 free parameters. The quality of the loading from each wave will be discussed next. Model fit and reliability measures will also be presented in each of the subsections below. Overall, model fit indices showed that a one-factor model fit the data well. Additionally, each of the indicators from the CBCL and SSRS measures loaded highly on the latent factor termed emotion dysregulation.

Wave 1. The one-factor model yielded adequate overall data-model fit, $X^{2}(324)=$ $511.85, p<0.001$. This absolute model fit index was significant, which likely indicates poor fit even if the model was properly specified. Given the large $d f$ relative to the $\mathrm{X}^{2}$ value, as well as the ordinal nature of the variables, data-model fit was further evaluated using the other fit 
indices. Based on additional fit indices, the one-factor model fit the data well, RMSEA=0.026 $(90 \% \mathrm{CI}=0.022-0.031), \mathrm{CFI}=0.935, \mathrm{TLI}=0.930$. Examination of the standardized residuals and modification indices (MI) did not indicate any localized misfit. The Mplus default for minimum MI values to print suggested modifications was 10.00 . According to the results, there were no values above this minimum. The $R^{2}$, along with the unstandardized and standardized (standardized on $x$ and $y$ ) factor loadings are presented in Table 12. The standardized factor loadings above .40 indicate that the 27 items derived from the CBCL and SSRS subscales of anxiety/depression, aggression, attention, and self-control were strongly related to the emotion dysregulation latent factor.

Table 12

Wave 1 Factor Loadings and $R^{2}$ for CFA Model of Emotion Dysregulation ( $n=833$ )

\begin{tabular}{cccccc}
\hline \multicolumn{5}{c}{ Wave 1 } \\
\hline Item \# & Unstandardized & S.E. & Standardized & S.E. & $R^{2}$ \\
\hline A1 & 1 & 0 & $0.448^{* *}$ & $0.058^{* *}$ & $0.201^{* *}$ \\
A2 & $1.467 * *$ & 0.222 & $0.658^{* *}$ & 0.042 & $0.433^{* *}$ \\
A3 & $1.605^{* *}$ & 0.219 & $0.720^{* *}$ & 0.036 & $0.518^{* *}$ \\
A4 & $1.432^{* *}$ & 0.188 & $0.642^{* *}$ & 0.043 & $0.412^{* *}$ \\
A5 & $1.771^{* *}$ & 0.232 & $0.794^{* *}$ & 0.032 & $0.631^{* *}$ \\
A6 & $1.795^{* *}$ & 0.209 & $0.805^{* *}$ & 0.028 & $0.647^{* *}$ \\
A7 & $1.707^{* *}$ & 0.220 & $0.765^{* *}$ & 0.025 & $0.585^{* *}$ \\
A8 & $0.666^{* *}$ & 0.174 & $0.298^{* *}$ & 0.059 & $0.089^{*}$ \\
A9 & $1.407^{* *}$ & 0.232 & $0.631^{* *}$ & 0.056 & $0.398^{* *}$ \\
A10 & $1.612^{* *}$ & 0.229 & $0.723^{* *}$ & 0.042 & $0.522^{* *}$ \\
A11 & $1.35^{* *}$ & 0.267 & $0.605^{* *}$ & 0.064 & $0.366^{* *}$ \\
A12 & $1.098^{* *}$ & 0.267 & $0.492^{* *}$ & 0.089 & $0.242^{*}$ \\
A13 & $1.684^{* *}$ & 0.215 & $0.755^{* *}$ & 0.042 & $0.570^{* *}$ \\
A14 & $0.637 * *$ & 0.152 & $0.285^{* *}$ & 0.071 & $0.081^{* *}$ \\
A15 & $1.419 * *$ & 0.198 & $0.636^{* *}$ & 0.067 & $0.404^{* *}$ \\
A16 & $1.618^{* *}$ & 0.231 & $0.725^{* *}$ & 0.035 & $0.526^{* *}$ \\
A17 & $1.668^{* *}$ & 0.234 & $0.748^{* *}$ & 0.036 & $0.559^{* *}$ \\
A18 & $1.626^{* *}$ & 0.195 & $0.729^{* *}$ & 0.031 & $0.531^{* *}$ \\
A19 & $1.766^{* *}$ & 0.237 & $0.792^{* *}$ & 0.029 & $0.627^{* *}$ \\
A20 & $1.453^{* *}$ & 0.182 & $0.651^{* *}$ & 0.043 & $0.424^{* *}$ \\
A21 & $1.022^{* *}$ & 0.261 & $0.458^{* *}$ & 0.072 & $0.210^{* *}$ \\
B1 & $-0.972 * *$ & 0.156 & $-0.436^{* *}$ & 0.059 & $0.190^{* *}$ \\
& & & & &
\end{tabular}




\begin{tabular}{cccccc}
\hline \multicolumn{5}{c}{ Wave 1 } \\
\hline Item \# & Unstandardized & S.E. & Standardized & S.E. & $R^{2}$ \\
\hline B2 & $-0.551^{*}$ & 0.187 & $-0.247 * *$ & 0.077 & 0.061 \\
B3 & $-0.762 * *$ & 0.146 & $-0.342 * *$ & 0.063 & $0.117 *$ \\
B4 & $-0.888 * *$ & 0.168 & $-0.398 * *$ & 0.061 & $0.159 * *$ \\
B5 & $-0.895 * *$ & 0.151 & $-0.401 * *$ & 0.045 & $0.161 * *$ \\
B6 & $-1.197 * *$ & 0.156 & $-0.536 * *$ & 0.052 & $0.288^{* *}$ \\
\hline$* * p<0.001 ; * p<0.05$ & & & &
\end{tabular}

To further assess the quality of the measurement model for emotion dysregulation, reliability measures were computed. Internal consistency as measured by Cronbach's $\alpha$ for the 27 emotion dysregulation items was 0.784 . Given the large number of indicators and inclusion of negatively loading items, coefficient $H$ was also calculated using the standardized loadings for each of the items. Results indicated high construct reliability for the emotion dysregulation latent factor at wave $1(H=0.952)$.

Wave 3. Evaluation of the overall data-model fit for the measurement model at wave 3 yielded similar results to the first wave, $\mathrm{X}^{2}(324)=523.28, p<0.001$, which likely indicated poor absolute fit even when the model was properly specified. However, additional fit indices showed that the one-factor model at wave 3 fit the data well, RMSEA=0.030 (90\% CI=0.025 0.034), $\mathrm{CFI}=0.914, \mathrm{TLI}=0.907$. Examination of the standardized residuals and modification indices (MI) did not indicate any localized poor fit. The $R^{2}$, along with the unstandardized and standardized (standardized on $x$ and $y$ ) factor loadings for wave 3 are presented in Table 13 . Similar to wave 1 results, standardized factor loadings above .40 indicate that the 27 items were strongly related to the emotion dysregulation latent factor. 
Table 13

Wave 3 Factor Loadings and $R^{2}$ for CFA Model of Emotion Dysregulation ( $\left.n=699\right)$

\begin{tabular}{|c|c|c|c|c|c|}
\hline \multicolumn{6}{|c|}{ Wave 3} \\
\hline Item \# & Unstandardized & S.E. & Standardized & S.E. & $R^{2}$ \\
\hline A1 & 1 & 0 & $0.849 * *$ & 0.061 & $0.255^{* *}$ \\
\hline $\mathrm{A} 2$ & $1.408 * *$ & 0.149 & $0.251 * *$ & 0.035 & $0.506 * *$ \\
\hline A3 & $1.426 * *$ & 0.162 & $0.765 * *$ & 0.038 & $0.518 * *$ \\
\hline A4 & $1.123 * *$ & 0.167 & $0.756 * *$ & 0.059 & $0.322 * *$ \\
\hline A5 & $1.592 * *$ & 0.184 & $0.710 * *$ & 0.034 & $0.646 * *$ \\
\hline A6 & $1.605 * *$ & 0.169 & $0.760 * *$ & 0.030 & $0.657 * *$ \\
\hline A7 & $1.682 * *$ & 0.187 & $0.745 * *$ & 0.028 & $0.721 * *$ \\
\hline A8 & $0.498 * *$ & 0.156 & $0.340 * *$ & 0.079 & 0.063 \\
\hline A9 & $1.516 * *$ & 0.157 & $0.647 * *$ & 0.046 & $0.586 * *$ \\
\hline A10 & $1.497 * *$ & 0.157 & $0.759 * *$ & 0.042 & $0.572 * *$ \\
\hline A11 & $1.405 * *$ & 0.156 & $0.726 * *$ & 0.052 & $0.503 * *$ \\
\hline A12 & $1.506 * *$ & 0.180 & $0.743 * *$ & 0.050 & $0.578 * *$ \\
\hline A13 & $1.474 * *$ & 0.174 & $0.824 * *$ & 0.049 & $0.554 * *$ \\
\hline A14 & $0.673 * *$ & 0.158 & $0.581 * *$ & 0.080 & $0.116 *$ \\
\hline A15 & $1.282 * *$ & 0.209 & $0.542 * *$ & 0.065 & $0.419 * *$ \\
\hline A16 & $1.503 * *$ & 0.170 & $-0.318 * *$ & 0.036 & $0.576^{* *}$ \\
\hline A17 & $1.437 * *$ & 0.173 & $-0.289 * *$ & 0.041 & $0.527 * *$ \\
\hline A18 & $1.471 * *$ & 0.160 & $-0.385 * *$ & 0.042 & $0.552 * *$ \\
\hline A19 & $1.631 * *$ & 0.196 & $-0.445 * *$ & 0.022 & $0.678 * *$ \\
\hline A20 & $1.151 * *$ & 0.217 & $-0.385 * *$ & 0.066 & $0.338 * *$ \\
\hline A21 & $1.073 * *$ & 0.150 & $-0.514 * *$ & 0.060 & $0.294 * *$ \\
\hline B1 & $-0.629 * *$ & 0.112 & $0.849 * *$ & 0.057 & $0.101 *$ \\
\hline B2 & $-0.572 * *$ & 0.126 & $0.251 * *$ & 0.059 & $0.083^{*}$ \\
\hline B3 & $-0.762 * *$ & 0.195 & $0.765 * *$ & 0.084 & $0.148 *$ \\
\hline B4 & $-0.881 * *$ & 0.185 & $0.756 * *$ & 0.068 & $0.198 * *$ \\
\hline B5 & $-0.762 * *$ & 0.161 & $0.710 * *$ & 0.066 & $0.148 *$ \\
\hline B6 & $-1.018 * *$ & 0.213 & $0.760 * *$ & 0.080 & $0.264 * *$ \\
\hline
\end{tabular}

Cronbach's $\alpha$ indicated that emotion dysregulation at wave 3 was highly reliable ( $\alpha=$ 0.808). Following the procedure from wave 1, further assessment of the quality of the measurement model using coefficient $H$ was calculated based on the reported standardized loadings. Results indicated high construct reliability for the emotion dysregulation construct at wave $3(H=0.961)$. 
Wave 4. At wave 4, overall data-model fit for the measurement model yielded similar results to the first two waves, $\mathrm{X}^{2}(324)=499.76, p<0.001$, indicating poor absolute fit. However, additional fit indices indicated good data-model fit during wave 4, RMSEA=0.028 $(90 \% \mathrm{CI}=0.023-0.033), \mathrm{CFI}=0.921, \mathrm{TLI}=0.914$. Examination of the standardized residuals and modification indices (MI) did not indicate any localized poor fit. The unstandardized and standardized (standardized on $x$ and $y$ ) factor loadings, along with the $R^{2}$ values for wave 4 are presented in Table 14. Similar to wave 1 and 3 results, standardized factor loadings above .40 indicate that the 26 out of the 27 items were strongly related to the emotion dysregulation latent factor. Factor loading for item A8 (i.e., fears certain animals, situations, or places, other than school) indicated that it was not a significant indicator of emotion dysregulation at wave 4 ( $\lambda=$ $\left.0.179, p=0.349 ; R^{2}=0.007, p=0.637\right)$. When considering post hoc model modifications, Bandalos and Finney (2010) recommended against the removal of non-significant paths ("trimming") for several reasons. First, obtaining replicable results may decrease when using the same sample to modify and test the new model. Second, the hypothesized model becomes datadriven rather than theory-driven. Finally, a re-specified model without the significant factor loadings are not in line with a priori specifications discussed in Chapter 3. Based on the authors' recommendations, a decision was made to retain the specified model, which included the nonsignificant loading for item A8.

Table 14

Wave 4 Factor Loadings and $R^{2}$ for CFA Model of Emotion Dysregulation (n=699)

\begin{tabular}{cccccc}
\hline \multicolumn{7}{c}{ Wave 4 } \\
\hline Item \# & Unstandardized & S.E. & Standardized & S.E. & $R^{2}$ \\
\hline A1 & 1 & 0 & $0.452^{* *}$ & 0.061 & $0.204 * *$ \\
A2 & $1.475^{* *}$ & 0.243 & $0.667 * *$ & 0.046 & $0.445^{* *}$ \\
A3 & $1.420^{* *}$ & 0.225 & $0.642^{* *}$ & 0.044 & $0.412^{* *}$ \\
A4 & $1.198^{* *}$ & 0.239 & $0.541^{* *}$ & 0.06 & $0.293^{* *}$
\end{tabular}




\begin{tabular}{|c|c|c|c|c|c|}
\hline \multicolumn{6}{|c|}{ Wave 4} \\
\hline Item \# & Unstandardized & S.E. & Standardized & S.E. & $R^{2}$ \\
\hline A5 & $1.873 * *$ & 0.245 & $0.847 * *$ & 0.029 & $0.717 * *$ \\
\hline A6 & $1.804 * *$ & 0.233 & $0.815^{* *}$ & 0.041 & $0.665^{* *}$ \\
\hline A7 & $1.811 * *$ & 0.222 & $0.819 * *$ & 0.028 & $0.67 * *$ \\
\hline A8 & $0.179 * *$ & 0.191 & 0.081 & 0.086 & 0.007 \\
\hline A9 & $1.695 * *$ & 0.273 & $0.766 * *$ & 0.056 & $0.587 * *$ \\
\hline A10 & $1.64 * *$ & 0.21 & $0.742 * *$ & 0.036 & $0.55 * *$ \\
\hline A11 & $1.623 * *$ & 0.212 & $0.734 * *$ & 0.048 & $0.538 * *$ \\
\hline A12 & $1.866 * *$ & 0.302 & $0.844 * *$ & 0.056 & $0.712 * *$ \\
\hline A13 & $1.528 * *$ & 0.207 & $0.691 * *$ & 0.048 & $0.477 * *$ \\
\hline A14 & $0.622 * *$ & 0.193 & $0.281 * *$ & 0.072 & $0.079 *$ \\
\hline A 15 & $1.165^{* *}$ & 0.181 & $0.526 * *$ & 0.068 & $0.277 * *$ \\
\hline A16 & $1.644 * *$ & 0.226 & $0.743 * *$ & 0.042 & $0.552 * *$ \\
\hline A17 & $1.666 * *$ & 0.232 & $0.753 * *$ & 0.032 & $0.567 * *$ \\
\hline A18 & $1.521 * *$ & 0.249 & $0.688 * *$ & 0.046 & $0.473 * *$ \\
\hline A19 & $1.651 * *$ & 0.243 & $0.746 * *$ & 0.045 & $0.557 * *$ \\
\hline A20 & $1.393 * *$ & 0.24 & $0.630 * *$ & 0.053 & $0.397 * *$ \\
\hline A21 & $1.202 * *$ & 0.231 & $0.544 * *$ & 0.06 & $0.295^{* *}$ \\
\hline B1 & $-0.786 * *$ & 0.194 & $-0.355 * *$ & 0.071 & $0.126^{*}$ \\
\hline B2 & $-0.695 * *$ & 0.142 & $-0.314 * *$ & 0.05 & $0.099 *$ \\
\hline B3 & $-1.167 * *$ & 0.129 & $-0.528 * *$ & 0.054 & $0.278 * *$ \\
\hline B4 & $-1.413 * *$ & 0.279 & $-0.639 * *$ & 0.056 & $0.408 * *$ \\
\hline B5 & $-1.039 * *$ & 0.239 & $-0.470 * *$ & 0.071 & $0.221 * *$ \\
\hline B6 & $-1.305 * *$ & 0.253 & $-0.590 * *$ & 0.07 & $0.348 * *$ \\
\hline
\end{tabular}

Cronbach's $\alpha$ for emotion dysregulation at wave 4 was 0.769 , which indicated high internal consistency among the 26 out of 27 items from the CBCL and SSRS. Results using coefficient $H$ indicated high construct reliability for the emotion dysregulation construct at wave $4(H=0.961)$.

Fit indices, along with reliability measures, indicated that the single factor measurement models at each wave was appropriate in representing emotion dysregulation. For wave 4 in particular, overall model fit and high reliability was found despite a non-significant loading for item A8. Based on these results, the single factor model was retained for subsequent analyses. 


\section{Longitudinal CFA}

Given that the measurement models of emotion dysregulation fit the data well at each time point, the next step evaluated construct stability across time. For this analysis, indicator variables for each were loaded onto the emotion dysregulation factor within the respective wave (i.e., items on wave 1 loaded on the wave 1 EdR factor). EdR factors were allowed to correlate across the three time points in order to assess stability or invariance across waves 1,3 , and 4 . The longitudinal CFA model was over-identified with 3,240 $d f$ and 246 free parameters.

Examination of the standardized residuals and modification indices (MI) did not indicate any localized misfit. According to the results, there were no values above the minimum MI value of 10.0. The unstandardized and standardized (standardized on $x$ and $y$ ) factor loadings, along with the $R^{2}$ values for all three waves are presented in Table 15. Similar to the cross-sectional results, standardized factor loadings above .40 indicate that the CBCL and SSRS items were strongly related to the emotion dysregulation latent factor, which was found to be consistent across time points. Similar to the wave 4 cross-sectional analyses, item A8 did not load significantly on EdR at wave 4. Following earlier procedures, this item was retained for the specified model. The correlations, covariance, and variance estimates are presented in Table 16, which indicated that the latent factor EdR was highly correlated across all waves. Emotion dysregulation across all 3 waves was found to be highly reliable ( 81 items; $\alpha=0.896$ ). A coefficient $H$ value of 0.985 indicated high construct or maximal reliability across all waves. 
Table 15

Factor Loadings and $R^{2}$ for CFA Model of Emotion Dysregulation across Waves 1, 3, and 4 (n=834)

\begin{tabular}{|c|c|c|c|c|c|c|c|c|c|}
\hline \multicolumn{4}{|c|}{ Wave 1} & \multicolumn{3}{|c|}{ Wave 3} & \multicolumn{3}{|c|}{ Wave 4} \\
\hline Item \# & Standardized & S.E. & $R^{2}$ & Standardized & S.E. & $R^{2}$ & Standardized & S.E. & $R^{2}$ \\
\hline A1 & $0.598 * *$ & 0.061 & $0.357 * *$ & $0.572 * *$ & 0.051 & $0.327 * *$ & $0.492 * *$ & 0.066 & $0.242 * *$ \\
\hline $\mathrm{A} 2$ & $0.651 * *$ & 0.048 & $0.424 * *$ & $0.689 * *$ & 0.045 & $0.475 * *$ & $0.628 * *$ & 0.05 & $0.395 * *$ \\
\hline A3 & $0.702 * *$ & 0.05 & $0.493 * *$ & $0.701 * *$ & 0.046 & $0.491 * *$ & $0.564 * *$ & 0.055 & $0.318 * *$ \\
\hline A4 & $0.638 * *$ & 0.057 & $0.407 * *$ & $0.588 * *$ & 0.06 & $0.345 * *$ & $0.521 * *$ & 0.064 & $0.271 * *$ \\
\hline A5 & $0.825 * *$ & 0.039 & $0.681 * *$ & $0.813^{* *}$ & 0.036 & $0.661 * *$ & $0.845^{* *}$ & 0.033 & $0.714 * *$ \\
\hline A6 & $0.785^{* *}$ & 0.041 & $0.617 * *$ & $0.839 * *$ & 0.034 & $0.705 * *$ & $0.778 * *$ & 0.051 & $0.605 * *$ \\
\hline A7 & $0.757 * *$ & 0.031 & $0.573 * *$ & $0.86^{* *}$ & 0.029 & $0.740 * *$ & $0.814 * *$ & 0.03 & $0.663 * *$ \\
\hline A8 & $0.269 * *$ & 0.071 & 0.072 & $0.211 *$ & 0.084 & $0.045 * *$ & 0.112 & 0.086 & 0.013 \\
\hline A9 & $0.695 * *$ & 0.073 & $0.483 * *$ & $0.735 * *$ & 0.054 & $0.540 * *$ & $0.723 * *$ & 0.063 & $0.523 * *$ \\
\hline A10 & $0.733 * *$ & 0.057 & $0.537 * *$ & $0.783^{* *}$ & 0.038 & 0.613 & $0.753 * *$ & 0.046 & $0.566 * *$ \\
\hline A11 & $0.563 * *$ & 0.079 & $0.317 * *$ & $0.672 * *$ & 0.06 & $0.452 * *$ & $0.734 * *$ & 0.048 & $0.539 * *$ \\
\hline A12 & $0.550 * *$ & 0.077 & $0.303 * *$ & $0.687 * *$ & 0.062 & $0.472 * *$ & $0.821 * *$ & 0.059 & $0.674 * *$ \\
\hline A13 & $0.700 * *$ & 0.057 & $0.491 * *$ & $0.746^{* *}$ & 0.057 & $0.556 * *$ & $0.695^{* *}$ & 0.055 & $0.483 * *$ \\
\hline A14 & $0.348 * *$ & 0.073 & $0.121 *$ & $0.305 * *$ & 0.09 & 0.093 & $0.266 * *$ & 0.072 & 0.071 \\
\hline A 15 & $0.642 * *$ & 0.082 & $0.412 * *$ & $0.706^{* *}$ & 0.068 & $0.499 * *$ & $0.619 * *$ & 0.071 & $0.383 * *$ \\
\hline A16 & $0.690 * *$ & 0.047 & $0.476^{* *}$ & $0.712 * *$ & 0.051 & $0.506 * *$ & $0.691 * *$ & 0.054 & $0.477 * *$ \\
\hline A17 & $0.759 * *$ & 0.04 & $0.577 * *$ & $0.708 * *$ & 0.043 & $0.501 * *$ & $0.755^{* *}$ & 0.031 & $0.57 * *$ \\
\hline A18 & $0.729 * *$ & 0.045 & $0.531 * *$ & $0.773 * *$ & 0.045 & $0.598 * *$ & $0.640 * *$ & 0.051 & $0.41^{* *}$ \\
\hline A19 & $0.826 * *$ & 0.035 & $0.682 * *$ & $0.820 * *$ & 0.025 & $0.672 * *$ & $0.746 * *$ & 0.05 & $0.557 * *$ \\
\hline A20 & $0.648 * *$ & 0.062 & $0.419 * *$ & $0.635^{* *}$ & 0.056 & $0.404 * *$ & $0.575^{* *}$ & 0.056 & $0.331 * *$ \\
\hline A21 & $0.397 * *$ & 0.096 & $0.157 *$ & $0.551 * *$ & 0.063 & $0.304 * *$ & $0.552 * *$ & 0.063 & $0.305 * *$ \\
\hline B1 & $-0.526 * *$ & 0.071 & $0.277 * *$ & $-0.280 * *$ & 0.06 & $0.078 *$ & $-0.320 * *$ & 0.083 & 0.102 \\
\hline B2 & $-0.189 *$ & 0.086 & 0.036 & $-0.308 * *$ & 0.074 & $0.095^{*}$ & $-0.246 * *$ & 0.05 & $0.061 *$ \\
\hline B3 & $-0.323 * *$ & 0.074 & $0.104 *$ & $-0.476 * *$ & 0.075 & $0.226 * *$ & $-0.467 * *$ & 0.061 & $0.218 * *$ \\
\hline B4 & $-0.396 * *$ & 0.079 & $0.157 *$ & $-0.484 * *$ & 0.075 & $0.234 * *$ & $-0.622 * *$ & 0.06 & $0.387 * *$ \\
\hline
\end{tabular}




\begin{tabular}{cccccccccc}
\hline \multicolumn{3}{c}{ Wave 1 } & \multicolumn{4}{c}{ Wave 3 } & \multicolumn{3}{c}{ Wave 4 } \\
\hline Item \# & Standardized & S.E. & $R^{2}$ & Standardized & S.E. & $R^{2}$ & Standardized & S.E. & $R^{2}$ \\
\hline B5 & $-0.454^{* *}$ & 0.048 & $0.206^{* *}$ & $-0.394^{* *}$ & 0.084 & $0.155^{*}$ & $-0.410^{* *}$ & 0.083 & $0.168^{*}$ \\
B6 & $-0.552^{* *}$ & 0.068 & $0.305^{* *}$ & $-0.536^{* *}$ & 0.070 & $0.287 * *$ & $-0.563 * *$ & 0.071 & $0.317^{* *}$ \\
\hline$* * p<0.001 ; * p<0.05$ & & & & & & & & &
\end{tabular}

Table 16

Correlation (lower half), Covariance (upper half), and Variance (diagonal) for Estimated Factor Scores

\begin{tabular}{llll}
\hline & EdRw1 & EdRw3 & EdRw4 \\
\hline EdRw1 & $0.357 * *$ & $0.205^{* *}$ & $0.173^{* *}$ \\
EdRw3 & $0.699 * *$ & $0.327^{* *}$ & $0.226^{* *}$ \\
EdRw4 & $0.663 * *$ & $0.841^{* *}$ & $0.242^{* *}$ \\
\hline$* * p<0.001$ & & &
\end{tabular}


The previous set of analyses to assess the fit and quality of the specified one-factor measurement model indicated that items from the CBCL and SSRS do measure emotion dysregulation for the 834 preschool-aged children in the sample. Given that the models were specified appropriately for the data, subsequent analyses employed the factor structure used in the CFA models above. The next section will cover results from the latent growth models (LGM), which examined growth trajectories of emotion dysregulation for the sample of maltreated preschool-aged children across the 3 waves of data collection. Correct specification and high reliability of the measurement models were important indicators in modeling growth in emotion dysregulation scores across time.

\section{Research Question 1}

The aim of research question 1 was to determine the functional form for emotion dysregulation development across the three waves of data collection for the NSCAW study. Latent growth curves can provide information on children's initial levels of dysregulation, as well as growth to and from those levels. In order to examine these growth parameters for emotion dysregulation, a series of latent growth models was selected and evaluated for proper data-model fit.

\section{Latent growth models of emotion dysregulation}

Latent growth models (LGMs) were selected to identify the growth trajectories of maltreated preschool-aged children's emotion dysregulation across the three waves of data collection from the NSCAW study. The series of growth analyses began with a second-order growth model as discussed in Chapter 3. Second-order growth models were specified to examine growth of the first-order level factors. In this case, the first-order factors were the emotion dysregulation construct measured by the items derived from the CBCL subscales (i.e., 
anxiety/depression, attention, aggression) and the SSRS self-control subscale. Subsequent analyses and the rationale for each selected growth models are discussed following the secondorder growth model.

First-order growth model: Factor scores. Factor-saved scores of emotion dysregulation at waves 1,3 , and 4 were employed as the repeated continuous indicators for the first-order growth model. These factor scores were derived using the regression method-the default method in Mplus from the longitudinal CFA model from the analysis discussed in the previous section. The latent growth parameters (i.e., for the intercept and $\beta$ for the slope) were employed as first-order factors to examine the growth trajectories of emotion dysregulation. Results indicated overall poor data-model fit, $\mathrm{X}^{2}(3)=89.758, p<0.001$, RMSEA $=0.186(90 \% \mathrm{CI}=$ $0.154-0.220), \mathrm{CFI}=0.639, \mathrm{TLI}=0.639, \mathrm{SRMR}=0.094$. Given the poor model fit, interpretation of the point estimates from the growth model may be inappropriate. A potential source of poor fit for the growth model may be the factor scores derived from the longitudinal CFA. Statistical methods such as structural equation modeling (SEM) require continuous or interval scales as a basic assumption in the observed data. Unfortunately, variances, covariances, and means of the measured variables lack meaning due to their ordinal nature (Koran \& Hancock, 2010). In order to address this limitation, a second-order growth model using ordinal indicators was selected following recommendations by Koran and Hancock (2010).

Second-order growth model: Ordinal indicators. Typical growth models using an SEM framework require the assumption that the measured or indicator variables are on an interval-level scale. This is particularly important when means, variances, and covariances are necessary parameter estimates to evaluate change over time, as well as adequacy of data-model fit. Unfortunately, observed variables on an ordinal scale require a different approach when 
measuring change using an SEM framework. In the case of ordinal data, the specified growth model predicts joint ordinal proportions (Mehta, Neale, \& Flay, 2004). Unlike data on an interval-level scale where means, variances, and covariances are estimated, ordinal repeated measures design consist of a multidimensional contingency table. Growth parameters (i.e., intercept and slope) were specified as the second-order factors that represent aspects of change on the first-order factors of emotion dysregulation. The first-order level of the model included the indicator items A1 through 21 and B1 through B6 for each time point. These were entered in Mplus syntax as categorical variables in order for the program to employ a Weighted Least Squares (WLSMV) estimation method. Growth parameter estimates were calculated by including all indicator variables and latent emotion dysregulation factors across the 3 waves. In order to address model identification problems from an unconstrained model, invariance constraints were included to ensure that thresholds for each indicator items were the same across the three measurement occasions.

To address the challenge of modeling ordinal data requiring interval-level scales, recommendations by Koran and Hancock (2010) were applied in the next stage of analyses. According to the authors, ordinal-level variables can be employed in an SEM framework by mapping them to some underlying unobserved continuous variable and modeling the unobserved variable using similar methods for continuous data. This proceeds in 3 steps where the first two involve calculations of polychoric correlations. For the third step, estimated parameters are then fit in the SEM model of interest.

The first two steps were conducted in a single process using the WLSMV estimation method in Mplus. During this step, a monotonic transformation process matched the density of the observed ordinal distribution with the density of the continuous latent factor distribution. 
Next, the scale of the latent factor must be set in order to define this transformation. Scaling was done by setting the ordinal variable thresholds to be invariant across the three time points (Koran \& Hancock, 2010). In addition to establishing threshold invariance, fixing the variance of the error terms to a constant (theta method) was done in order to achieve model identification (Koran \& Hancock, 2010; Muthén \& Asparouhov, 2002). In other words, this stage of the process established a longitudinal CFA model with constraints on the threshold and error variances in order to create a linear composite of the ordinal indicators. Finally, a latent growth model using an SEM framework was fit to the data.

Based on the absolute fit index, the null model should be retained, $X^{2}(3264)=3652.536$, $p<0.001$. Considering other criteria, however, there was indication that adequate model fit was obtained, RMSEA $=0.012(90 \% \mathrm{CI}=0.009-0.014), \mathrm{CFI}=0.905, \mathrm{TLI}=0.906$. Figure 7 shows the specification of the second-order ordinal growth model, along with the estimated parameters. Point estimates showed no significant difference in mean values for the initial level ( $\alpha=0.043, p$ $=0.632)$ and growth $(\beta=-0.002, p=0.453)$ for emotion dysregulation. However, the covariation between these intercept and slope parameters was significant $\left(\operatorname{covar}_{\alpha \beta}=0.002, p<0.05\right)$, indicating that the two growth parameters were related. There appeared to be significant variability in the initial levels of emotion dysregulation $\left(v a r_{\alpha}=0.175, p<0.05\right)$. Significant residual variances for the emotion dysregulation factor at each time point were significant $\left(E d R_{\text {res }}=0.105, p<0.001\right)$, indicating potential problems with the selected model when accounting for measurement error. The emotion dysregulation scores were estimated by the longitudinal CFA portion of the model, which became the first-order level that was evaluated by the growth portion of the overall model. 


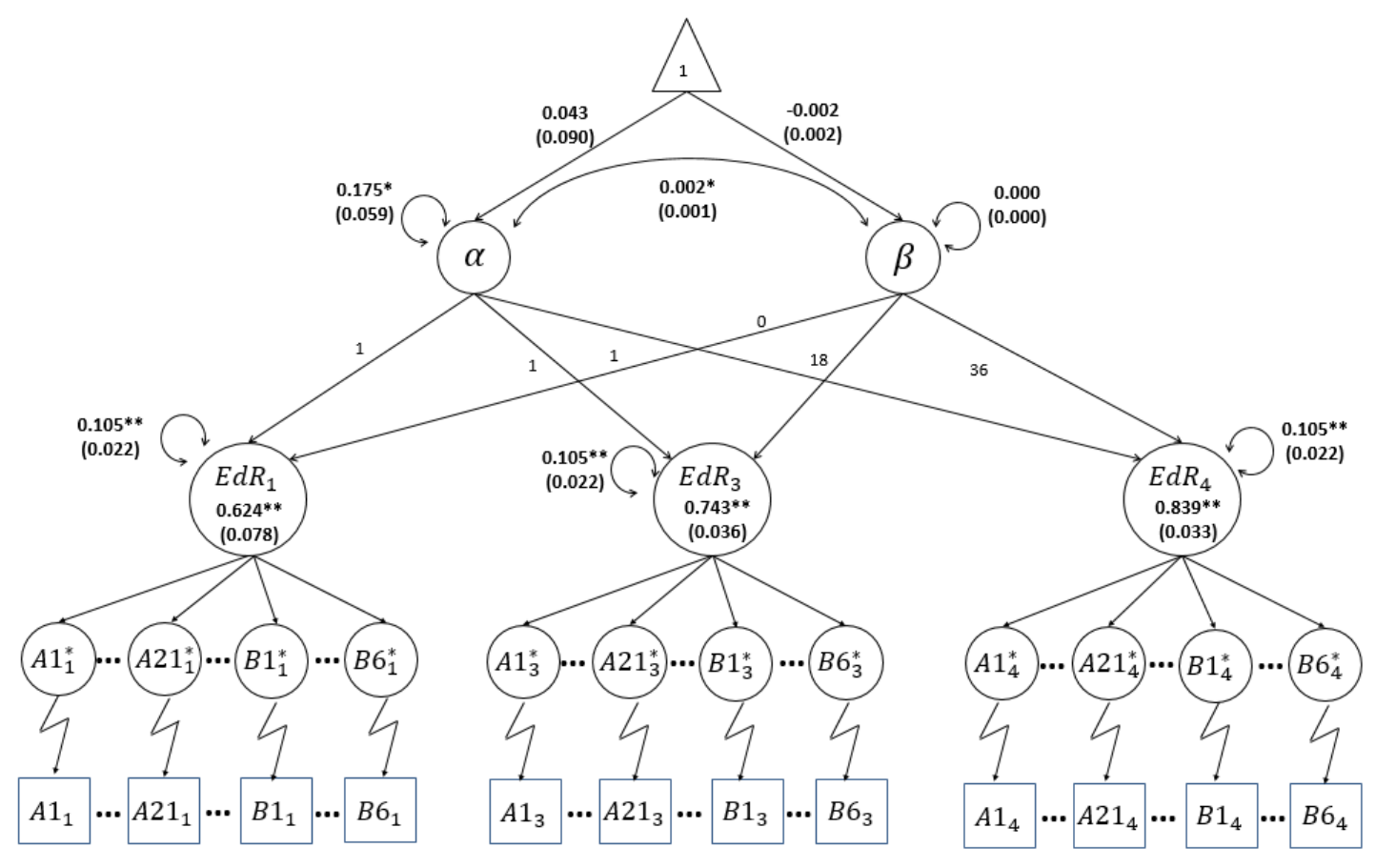

Figure 7. Second-order growth model with ordinal indicators. Jagged lines represent the link function that provides an underlying latent continuum transformation of the ordinal indicators. Estimated means and standard errors (in parentheses) are shown $(\mathrm{n}=834)$

Note. ${ }^{* *} p<0.001 ; * p<0.05$

Despite model convergence and an indication of adequate model fit, interpretation of the estimated scores for emotion dysregulation is problematic. These scores were derived through a probit link (represented by the jagged arrows in Figure 7) between the ordinal indicators into a continuous latent factor for each ordinal indicator. These transformed latent factors were then employed as indicators of emotion dysregulation. The growth model was then imposed on the resulting emotion dysregulation construct once the transformation was completed. This analysis of growth in emotion dysregulation assumes that the scale for the latent factor indicators should have been transformed from the ordinal indicators. This underlying continuous structure then should have been translated into the latent construct of emotion dysregulation. However, given that the ordinal indicator items were measured on a 3-level polytomous response, it is difficult to interpret the resulting scale. For example, the EdR score for wave 1 was 0.624 . Does this value 
fall within a standardized continuous value of -2 to +2 given the constrained thresholds imposed on the measurement portion of the model? Or can this value be interpreted on an absolute level indicating that the emotion dysregulation score was closer to 1 from the original $0,1,2$ scale?

Mehta et al. (2004) examined growth over time for a single ordinal item measured repeatedly across 3 time points, which made estimated parameters easily interpretable based on the direct transformation of the single ordinal item into meaningful thresholds. In this case, however, the coarseness of the ordinal items (i.e., 3 response categories) were collapsed into a single latent construct, making it challenging to interpret the resulting score. As such, the results from the growth parameters are also in question. That is, problems with interpreting the estimated factor scores for emotion dysregulation may have been transmitted to the imposed growth model portion of the model. Thus, the next set of analyses attempted to address the interpretability of the latent factor by reducing the polytomous responses of the ordinal indicators to dichotomous scores.

Second-order growth model: Dichotomous indicators. To see whether interpretation of the estimated latent scores can be improved upon, responses on the 3-point Likert scale were recoded into dichotomous responses. Each of the 27 indicator items at each of the 3 time points yielded answers that indicated the presence (1) or absence (0) of problem behaviors. After recoding, procedures similar to the second-order ordinal growth model were followed, which included specification of the measurement model with threshold invariance and theta parameterization method imposed.

Results indicated poor overall data-model fit, $\mathrm{X}^{2}(3210)=3446.706, p<0.001$, RMSEA=0.009 $(90 \% \mathrm{CI}=0.006-0.012), \mathrm{CFI}=0.890, \mathrm{TLI}=0.889$. See Table 17 for point estimates of the growth model. Similar to the ordinal growth model, residual variances for 
emotion dysregulation at each time point were significant $(0.087, p<0.001)$, possibly indicating problems with the selected model. Growth parameters were not significant $(\alpha=0.127, p=$ 0.092; $\beta=-0.001, p=0.633$ ), nor was there significant covariation between these two parameters $\left(\operatorname{covar}_{\alpha \beta}=0.001, p=0.385\right)$.

Table 17

Point Estimates of the Second-Order Growth Model with Dichotomous Indicators

\begin{tabular}{lcccc}
\hline & Means & Variances & Covariances & Residual Variances \\
\hline Intercept & $0.127(\mathrm{p}=0.092)$ & $0.182 *(\mathrm{p}=0.012)$ & $0.001(\mathrm{p}=0.385)$ & -- \\
Slope & $-0.001(\mathrm{p}=0.633)$ & $0.000(\mathrm{p}=0.097)$ & & -- \\
EdRw1 & -- & -- & -- & $0.087 * *$ \\
EdRw3 & -- & -- & -- & $0.087 * *$ \\
EdRw4 & -- & -- & -- & $0.087 *$ \\
$* * p<0.001 * * p<0.05$ & & &
\end{tabular}

Based on the preceding analysis, the shift from polytomous to dichotomous responses resulted in a poor fitting model when compared with the growth model of ordinal indicators. Given similar procedures to the ordinal model, the underlying continuous factor scores in this model were scaled based on the dichotomous indicators. Thus, problems with interpretability of the factor scores remain and difficulty with accurate interpretation of the growth parameters continue to be problematic. Given the problems with factor scores and categorical (ordinal and dichotomous) indicators in modeling growth, the final step in this section incorporated summed scores following similar procedures employed by Ayer et al. (2009) in their analysis of the dysregulation profile of the CBCL.

First-order growth model: Summed scores. For this next set of analysis, a first-order latent growth model was fit to the data using summed scores. Summed scores were selected for this procedure to address the problem of interpretability of the emotion dysregulation scores. By employing summed scores, the repeated measures were continuous, with interval-level data 
making growth parameters easier to interpret. The factor structure for emotion dysregulation was validated using the cross-sectional and longitudinal CFA models in earlier analyses. With the single factor measurement model fitting the data well, scores were summed across the 27 ordinal indicators. Results indicated overall poor data-model fit, $\mathrm{X}^{2}(3)=66.468, p<0.001$, RMSEA $=0.097(90 \% \mathrm{CI}=0.065-0.133), \mathrm{CFI}=0.628, \mathrm{TLI}=0.628, \mathrm{SRMR}=0.133$. Because of the poor data-model fit, point estimates for the growth model were not evaluated.

\section{Possible Sources of Model Misfit}

Results from the series of growth model analyses consistently showed poor data-model fit when attempting to model the growth trajectory of maltreated preschool-aged children's emotion dysregulation. This makes it difficult to interpret the accuracy of the growth parameters and to say with certainty that children's development of emotion dysregulation was changing or not. The selection of growth models may be problematic when imposed on data with ordinal data. The lack of significant results could be a function of the coarseness of the indicators with only 3 distinct response categories (i.e., 3-point Likert scale) and the availability of only 3 time points. Despite attempts at adjusting the growth models to accommodate ordinal data, the resulting factor scores may not provide enough variability to model growth. Additionally, the linear composite created by the factor analysis assumed that the unidimensionality of the latent factor was correct. Thus, further analyses of the separate indicator items need to be considered.

Implications for these results, as well as future directions will be further discussed in Chapter 5. Given that the significant growth trend was not available to answer the next research question, alternate models were selected to undergo subgroup analyses. These will be covered next. 


\section{Research Question 2}

The second research question aimed to identify unobserved groups based on differences in growth trajectories of maltreated preschool-aged children. Given the lack of convergence and resulting model misfit for the growth models selected in answering the first research question, a growth mixture model was not appropriate for this section. To answer research question 2, alternate latent variable mixture models were selected for subgroup analyses. Specifically, latent class analysis (LCA) and latent transition analysis (LTA) were employed to identify the unobserved groups of maltreated children based on their emotion dysregulation pattern.

\section{Latent Class Analysis}

Unlike the preceding methods of confirmatory factor analyses (CFA) and structural equation modeling (SEM) that require observed variables to be on a continuous scale, LCA is traditionally employed when analyzing categorical data (Samuelsen \& Dayton, 2010). LCA is a method that classifies cases into known (specified a priori) or unknown (latent) groups (see Figure 8). Cases that have similar response patterns on the categorical measured items are then classified into unobserved or latent classes based on probabilistic estimation methods (Samuelsen \& Dayton, 2010). For this study, LCA was employed in an exploratory manner to examine response patterns on the 27 categorical items that are intended to measure emotion dysregulation. Cross-sectional LCA models were specified first, which was then followed by the LTA model. 


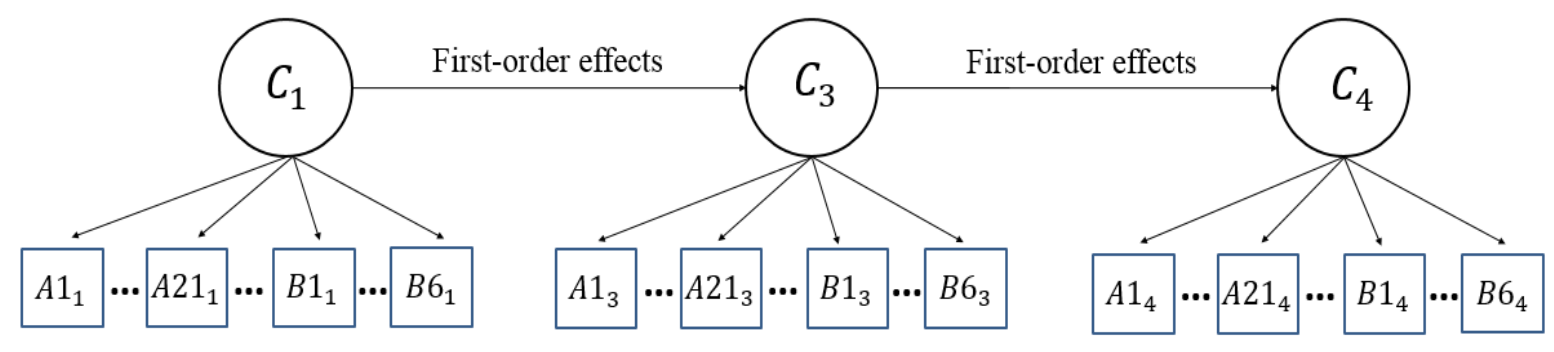

Figure 8. Latent class model across 3 time points with first order effects.

Note. Adapted from Nylund (2007).

Wave 1 latent class analysis: Dichotomous indicators. For the first model, wave 1 scores on the 27 indicator items were dichotomized and analyzed using Mplus. During model estimation, the following error message was displayed: “THE CHI-SQUARE TEST CANNOT BE COMPUTED BECAUSE THE FREQUENCY TABLE FOR THE LATENT CLASS

INDICATOR MODEL PART IS TOO LARGE.” Chi-square $\left(\mathrm{X}^{2}\right)$ values were calculated using Pearson statistic (based on differences between observed and expected frequencies) and is often used to evaluate absolute model fit. This situation is known as sparse data, which often occurs when the number of observed categorical variables is large.

According to Magidson and Vermunt (2004), when sparse data is present in the analysis, the $\mathrm{X}^{2}$ distribution should not be used because the total number of cells in the frequency table will be large relative to sample size, which results in many empty cells (i.e., sparse data). Thus, the resulting $p$-value for the test of model fit will not be appropriate because the $L^{2}$ will not be replicated. Instead of the model $\mathrm{X}^{2}$, alternative fit indices were selected to evaluate model fit based on the authors' recommendations. Specifically, AIC, BIC, and Adjusted BIC values were used for model comparison where smaller values indicated better fit. Entropy values were used in order to assess the classification performance of the model where values closer to 1 indicated better class separation. Finally, likelihood ratio tests (i.e., LMR Adjusted LRT and VLMR LRT) 
were used to compare a $(k-1)$-class null model with a $k$-class alternative (less restrictive) model. A statistically significant $p$-value suggests that the $k$-class model fits the data better than a model with $k-1$ fewer classes. Results of the test of model fit is presented in Table 18.

Given that this was an exploratory analysis with a descriptive rather than inferential purpose, a 2-class model was selected as the best-fitting model despite the non-significant LRT values. Selection of the 2-class model was based on the smaller AIC values relative to the 1-class model. Entropy values for the 2-class model was slightly higher than the 3-class model, and thus the 2-class model was retained. There were 398 (48\%) children classified under latent class 1 (i.e., emotionally dysregulated) and 435 (52\%) children classified under latent class 2 (i.e., emotionally regulated). Graphical results of the latent classes and probability of occurrence for each item are shown in Figure 9. Item probabilities for the LCA models at each wave are presented in Appendix K. The probability of endorsing these items that indicated problem behaviors are highlighted based on class membership.

Table 18

Model Fit Index for Wave 1 LCA Model $(n=833)$

\begin{tabular}{ccccccc}
\hline $\begin{array}{c}\text { No. of } \\
\text { Classes }\end{array}$ & AIC & BIC & Adjusted BIC & LRT & Entropy & VLMR LRT \\
\hline 1 & 27073.967 & 27201.543 & 27115.800 & $\begin{array}{c}-- \\
2847.721\end{array}$ & -- & - \\
2 & 24267.123 & 24527.000 & 24352.338 & $\begin{array}{c}(\mathrm{p}=0.3322) \\
595.015\end{array}$ & 0.868 & $1 \mathrm{v} 2=\mathrm{ns}$ \\
3 & 23724.948 & 24117.126 & 23853.547 & $\begin{array}{c}\mathrm{p}=0.8244) \\
311.347\end{array}$ & 0.841 & $2 \mathrm{v} 3$ classes $=\mathrm{ns}$ \\
4 & 23467.948 & 23992.426 & 23639.929 & $(\mathrm{p}=0.7304)$ & 0.834 & $3 \mathrm{v} 4$ classes $=\mathrm{ns}$ \\
\hline$* p<0.001 ; * p<0.05$ & & & &
\end{tabular}




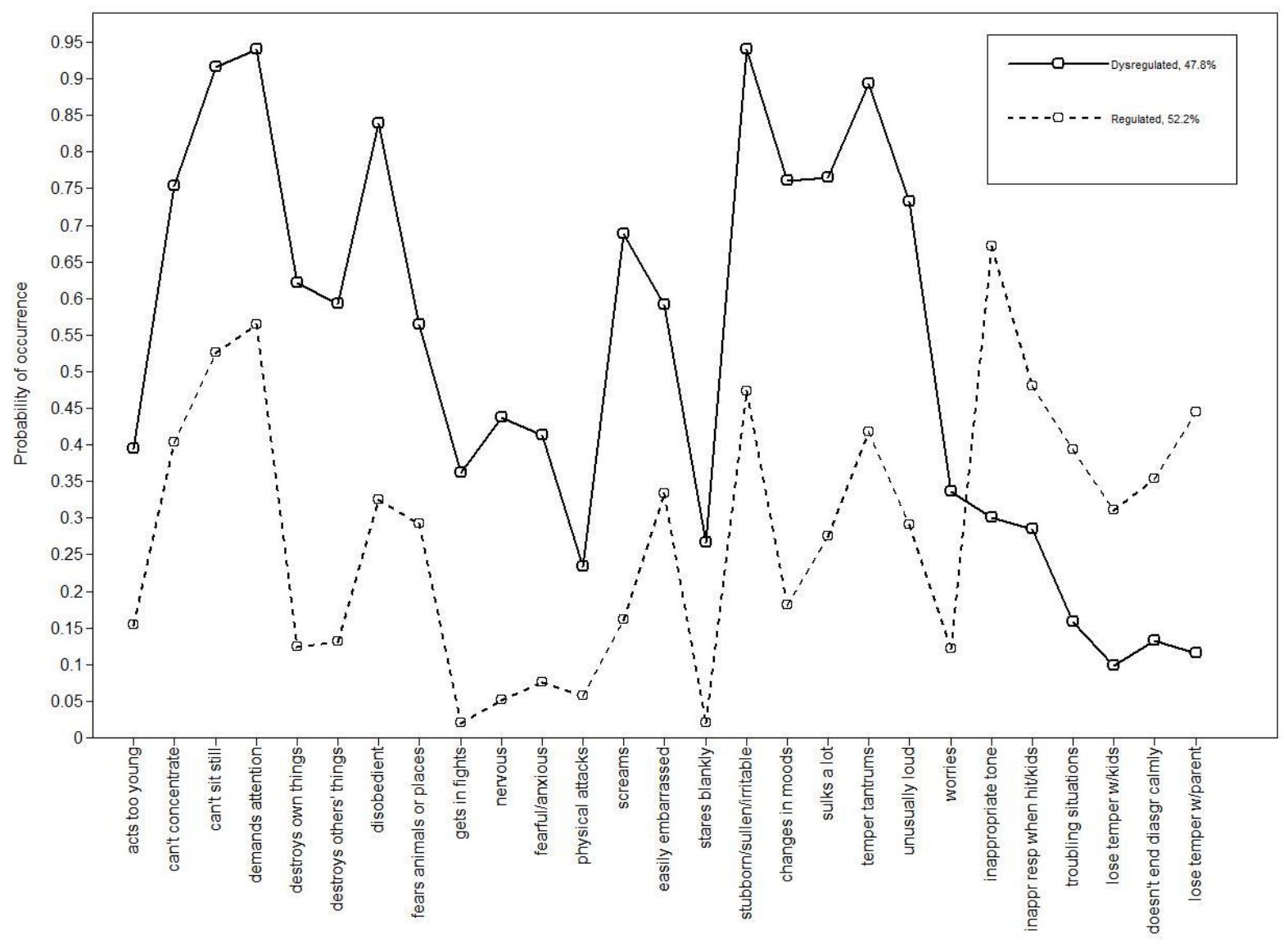

Figure 9. Profiles for the 2-class model showing emotionally regulated and dysregulated maltreated children at wave 1 .

Wave 3 latent class analysis: Dichotomous indicators. Convergence problems occurred starting with the specification of a 4-class solution. These may be due to problems with replication of the loglikelihood. Although the entropy value for the 4-class model was higher than the 2-class model, the 2-class model was selected as the best-fitting model. The 2-class model was retained due to a fairly high entropy value and lack of convergence problems.

Results of the wave 3 cross-sectional LCA indicated that a 2-class model fit the data well. Model selection criteria are presented in Table 19. There were 363 (52\%) children classified under latent class 1 (i.e., emotionally dysregulated) and 335 (48\%) children classified under 
latent class 2 (i.e., emotionally regulated). Figure 10 shows the items that have a higher probability of endorsement by children who were classified as emotionally dysregulated. Table 19

Model Fit Index for Wave 3 LCA Model $(n=699)$

\begin{tabular}{ccccccc}
\hline $\begin{array}{c}\text { No. of } \\
\text { Classes }\end{array}$ & AIC & BIC & Adjusted BIC & $\begin{array}{c}\text { LMR Adjusted } \\
\text { LRT }\end{array}$ & Entropy & VLMR LRT \\
\hline 1 & 23324.981 & 23447.882 & 23362.092 & $\begin{array}{c}-- \\
2759.902 \\
(\mathrm{p}=0.144)\end{array}$ & -- & - \\
2 & 20606.030 & 20856.261 & 20681.625 & $\begin{array}{c}646.041 \\
(\mathrm{p}=0.7442) \\
368.182\end{array}$ & 0.883 & 2 v 3 classes $=\mathrm{ns}$ \\
3 & 20012.466 & 20390.087 & 20126.546 & & \\
4 & 19698.276 & 20203.288 & 19850.841 & $(\mathrm{p}=0.8156)$ & 0.918 & $3 \mathrm{v} 4$ classes $=\mathrm{ns}$ \\
\hline$* * p<0.001 ; * p<0.05$ & & & &
\end{tabular}

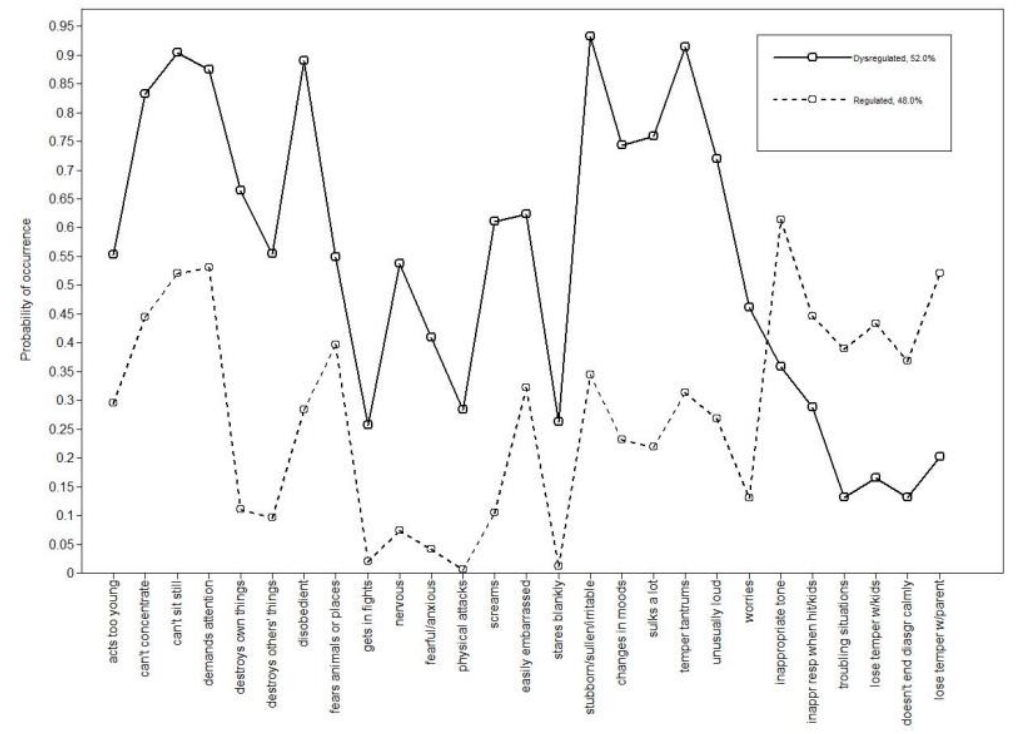

Figure 10. Profiles for the 2-class model showing emotionally regulated and dysregulated maltreated children at wave 3 . 
Wave 4 latent class analysis: Dichotomous indicators. Results of the wave 4 crosssectional LCA indicated that a 2-class model fit the data well. These results indicate that there appears to be stability in number of classes across the three waves. Moreover, high entropy values for the 2-class solution across the three time points indicate that the model distinguishes between the classes very well. Model selection criteria are presented in Table 20. There were 363 $(52 \%)$ children classified under latent class 1 (i.e., emotionally dysregulated) and 335 (48\%) children classified under latent class 2 (i.e., emotionally regulated). Figure 11 shows the items that have a higher probability of endorsement by children who were classified as emotionally dysregulated at wave 4 .

Table 20

Model Fit Index for Wave 4 LCA Model $(n=700)$

\begin{tabular}{ccccccc}
\hline $\begin{array}{c}\text { No. of } \\
\text { Classes }\end{array}$ & AIC & BIC & Adjusted BIC & $\begin{array}{c}\text { LMR Adjusted } \\
\text { LRT }\end{array}$ & Entropy & VLMR LRT \\
\hline 1 & 23656.390 & 23779.269 & 23693.539 & $\begin{array}{c}-- \\
2682.048\end{array}$ & -- & -- \\
2 & 21015.721 & 21266.031 & 21091.394 & $\begin{array}{c}(\mathrm{p}=0.6591) \\
595.015\end{array}$ & 0.879 & $1 \mathrm{v} 2=\mathrm{ns}$ \\
3 & 20429.789 & 20807.528 & 20543.987 & $\begin{array}{c}(\mathrm{p}=0.8244) \\
373.225\end{array}$ & 0.910 & $2 \mathrm{v} 3$ classes $=\mathrm{ns}$ \\
4 & 20110.529 & 20615.699 & 20263.252 & $(\mathrm{p}=0.7100)$ & 0.911 & $3 \mathrm{v} 4$ classes $=\mathrm{ns}$ \\
\hline$* p<0.001 ; * p<0.05$ & & & &
\end{tabular}




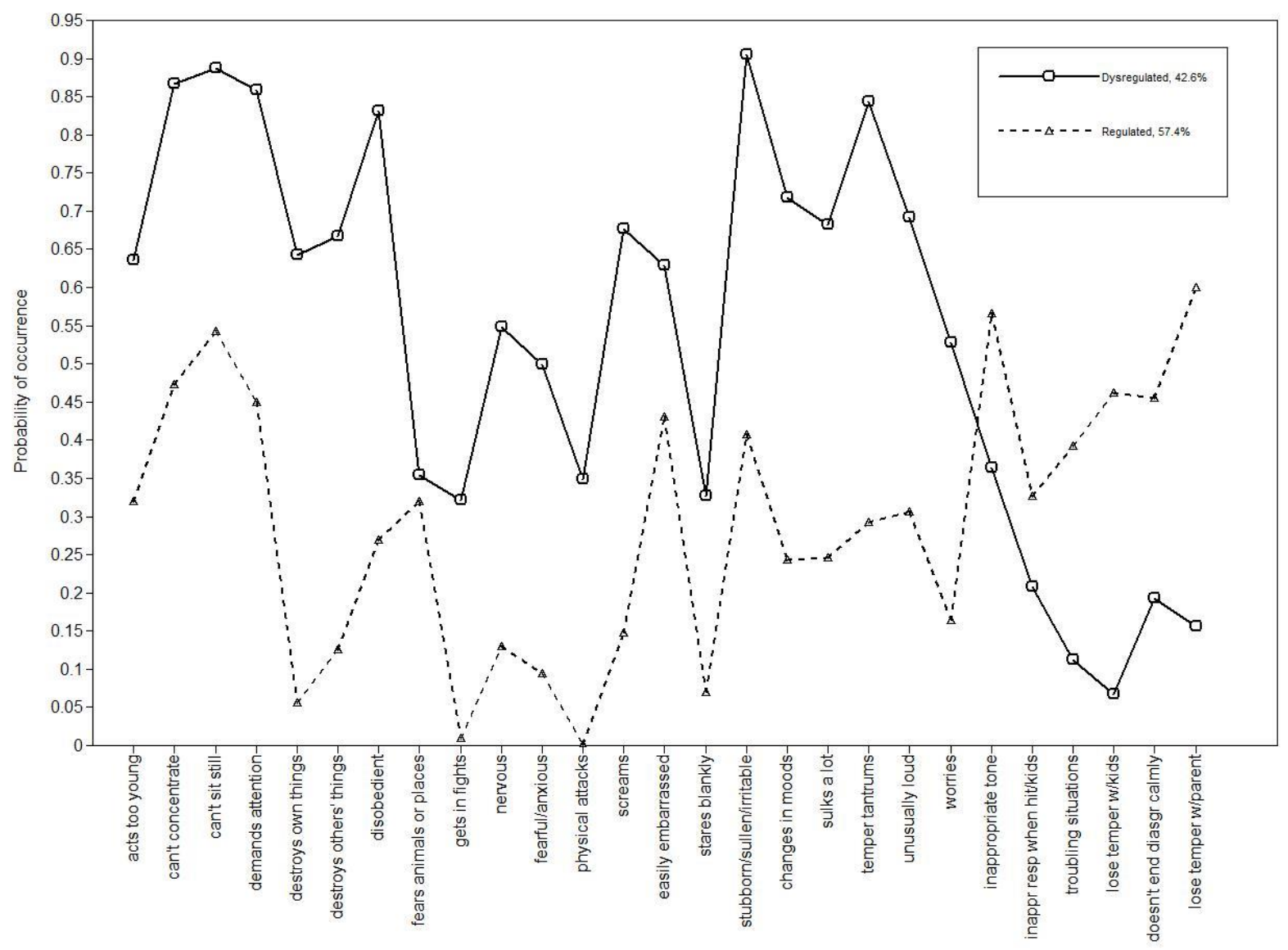

Figure 11. Profiles for the 2-class model showing emotionally regulated and dysregulated maltreated children at wave 3 .

Upon examination of the cross-sectional longitudinal data, it appeared that there were a number of children that may have changed classes over time. This can be seen in the shifting proportion in each class at each cross-sectional wave (see Figure 12 and Table 21). The cross-sectional LCA models also appeared to classify children with clear separation between classes consistently over time (see Table 22). These results provide justification for proceeding to the next step and employing an LTA model where threshold invariance is employed. 
Table 21

Cross-sectional Comparison of Class Counts \& Proportions of Children in Each Class

\begin{tabular}{lcccccc}
\hline & \multicolumn{2}{c}{ Wave 1 $(n=833)$} & \multicolumn{2}{c}{ Wave 3 $(n=699)$} & \multicolumn{2}{c}{ Wave $4(n=700)$} \\
\hline & Count & Proportion & Count & Proportion & Count & Proportion \\
\cline { 2 - 7 } dysregulated class & 398 & 0.478 & 363 & 0.520 & 298 & 0.426 \\
regulated class & 435 & 0.522 & 336 & 0.480 & 402 & 0.574 \\
\hline
\end{tabular}

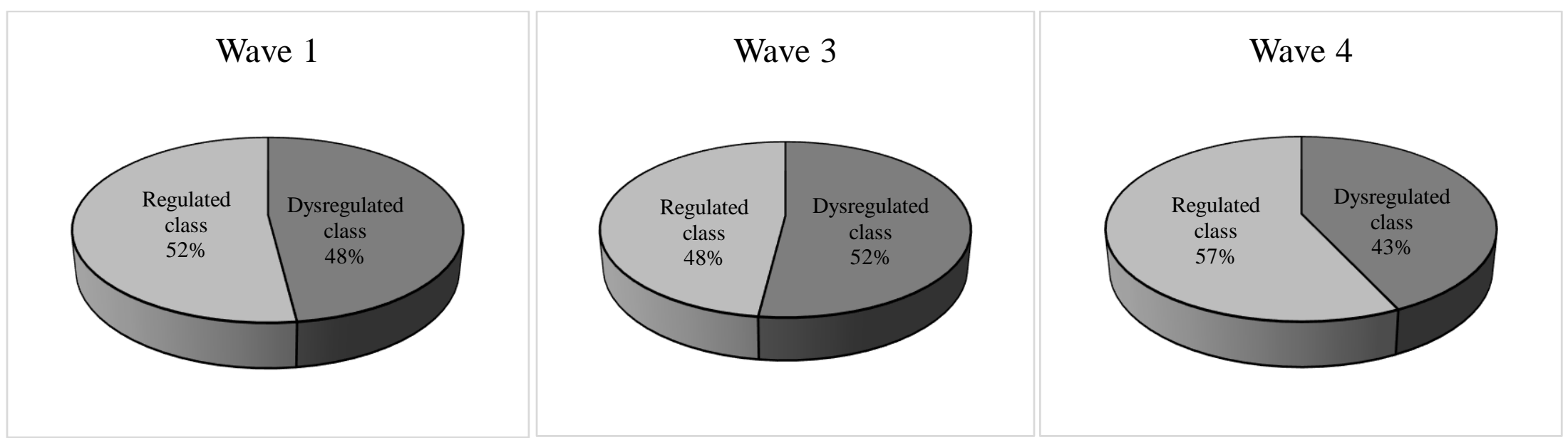

Figure 12. Proportions of each latent class at each wave. 
Table 22

Cross-sectional Comparison of Class Selection Probabilities

\begin{tabular}{ccccccc}
\hline \multicolumn{9}{c}{ Classification Table } \\
\hline & EdR & ER & EdR & ER & EdR & ER \\
\cline { 2 - 7 } & 0.961 & 0.039 & 0.978 & 0.022 & 0.957 & 0.043 \\
EdR & 0.033 & 0.967 & 0.028 & 0.972 & 0.031 & 0.969 \\
\hline ER &
\end{tabular}

Note. EdR = Emotionally Dysregulated; ER = Emotionally Regulated

\section{Latent Transition Analysis}

Given that the original aim of this question was to examine underlying subgroups in the development of maltreated children's emotion dysregulation, a latent transition analytic (LTA) model was chosen. According to Rindskopf (2010), LTA models are often used when latent categorical constructs are employed in more than 2 time points, parameters of interest are often included that assess initial status and transition probabilities from one latent class into another, and observed categorical variables are imperfect indicators of the hypothesized latent construct, which in this case was emotion dysregulation. A Second-order effect model (see Figure 13) was selected in order to take into account the impact that wave 1 classification might have on wave 3 based on the developmental nature of the inquiry (Nylund, 2007). Additionally, the selected LTA model allowed for backward transitions between classes to better model the hypothesized experiences that young maltreated children have when experiencing context-level factors specific to contact with CPS. That is, given the possible changes in placement and quality of the caregiving environment, children's latent classification may move from being emotionally regulated to emotionally dysregulated or vice versa. The flexibility of the LTA model allowed for these backward class transitions. 


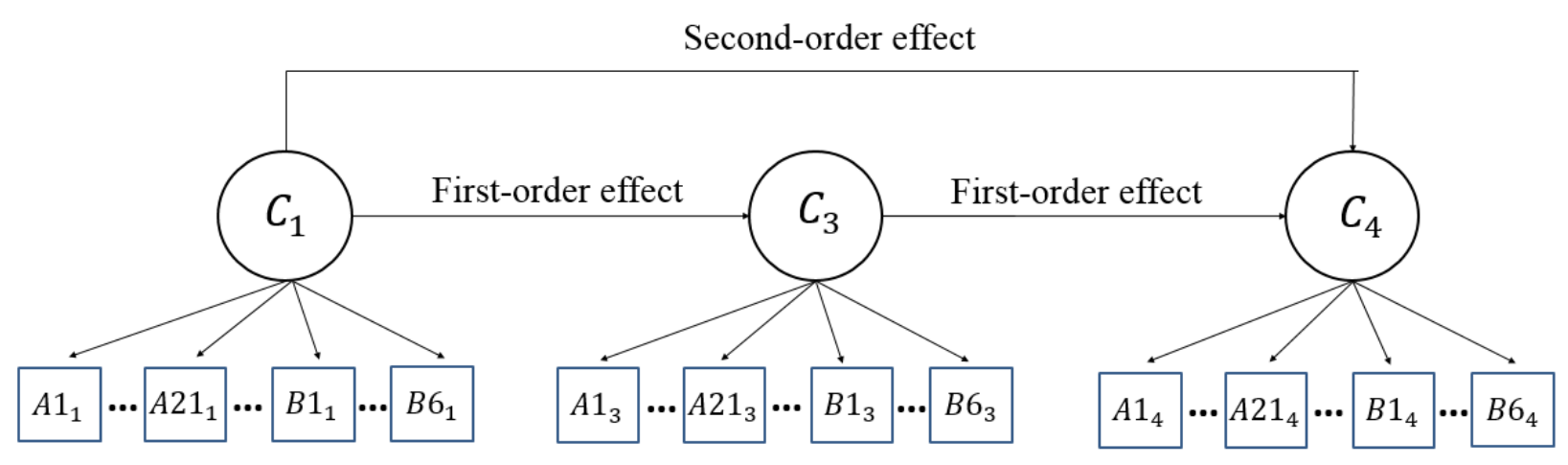

Figure 13. Second-order effect model for latent transition analysis

Based on results attained from the cross-sectional LCA, a 2-class model was chosen to be modeled across the 3 waves of data collection. Model fit indices are presented in Table 23. A majority of the children in this sample had a higher probability of belonging to the dysregulated emotion latent class when transitioning from wave 1 to 3 and from wave 3 to 4 (74\% and $75.5 \%$ respectively). Table 24 displays the transition probabilities for maltreated children's emotion regulation/dysregulation classes across time. A similar pattern was present for children with a higher probability of belonging to the regulated emotion latent class when transitioning from wave 1 to 3 and from wave 3 to 4 ( $73 \%$ and $86.8 \%$ respectively). These results provide evidence that a majority of the children remained stable within their class membership. An additional $27 \%$ of children transitioned from the regulated emotion class to the dysregulated emotion class from wave 1 to 3 , and $13.2 \%$ from wave 3 to 4 . Finally, $26 \%$ of the children went from belonging to the dysregulated emotion latent class to the regulated emotion latent class from wave 1 to 3 and $24.5 \%$ from wave 3 to 4 . Taken together, these transition probabilities indicated an increase in class transitions from wave 1 to 3 compared to the class transition from wave 3 to 4 . 
Table 23

Model Fit Index for LTA Model $(n=834)$

\begin{tabular}{ccccc}
\hline $\begin{array}{c}\text { No. of } \\
\text { Classes }\end{array}$ & AIC & BIC & Adjusted BIC & Entropy \\
\hline 1 & 78904.68 & 79273.33 & 79025.63 & -- \\
2 & 73370.12 & 73648.97 & 73461.60 & 0.884 \\
3 & 71617.30 & 72033.21 & 71753.76 & 0.849 \\
\hline
\end{tabular}

Table 24

Transition Probabilities across Waves

\begin{tabular}{llrr}
\hline \multicolumn{3}{c}{ Transition probabilities } \\
\cline { 3 - 4 } & & \multicolumn{2}{c}{ Wave 3 } \\
\cline { 3 - 4 } Wave 1 & Dysregulated & Regulated \\
& Regulated & 0.740 & 0.260 \\
& Regulated & 0.270 & 0.730 \\
\hline
\end{tabular}

\section{Transition probabilities}

\begin{tabular}{llrr}
\hline & & \multicolumn{2}{c}{ Wave 4 } \\
\cline { 3 - 4 } Wave 3 3 & Dysregulated & 0.755 & 0.245 \\
& Regulated & 0.132 & 0.868 \\
\hline
\end{tabular}

There were 8 transition patterns that emerged from the data (see Figure 14). A majority of the children belonged to stable classes across waves 1, 3, and 4 . That is, $31 \%$ were classified as emotionally dysregulated - stable and $34 \%$ were classified as emotionally regulated - stable due to consistent classification across all waves. The short-term regulating trend developmental pattern included children (5\%) who had 2 previous waves of belonging to the emotionally dysregulated class and transitioning to the regulated class at wave 4 . The short-term dysregulating trend pattern on the other hand, included children (3\%) who had 2 previous waves of being classified as regulated and transitioning to the dysregulated class at wave 4 . The 
regulated short-term (4\% of children) and dysregulated short-term (7\%) developmental patterns included children who were either regulated or dysregulated at wave 1 , transitioned to an alternate class at wave 3, then returning to the same class at wave 4 . Finally, the regulating trend - long-term developmental pattern included children (9\%) who were dysregulated at wave 1, then transitioned to the regulated class at waves 3 and 4, while the dysregulating trend - longterm pattern included children (7\%) who were classified as regulated at wave 1 and transitioned to the dysregulated class at waves 3 and 4 . These 8 distinct patterns provide evidence of heterogeneity in the development of young maltreated children's emotion regulation skills. 

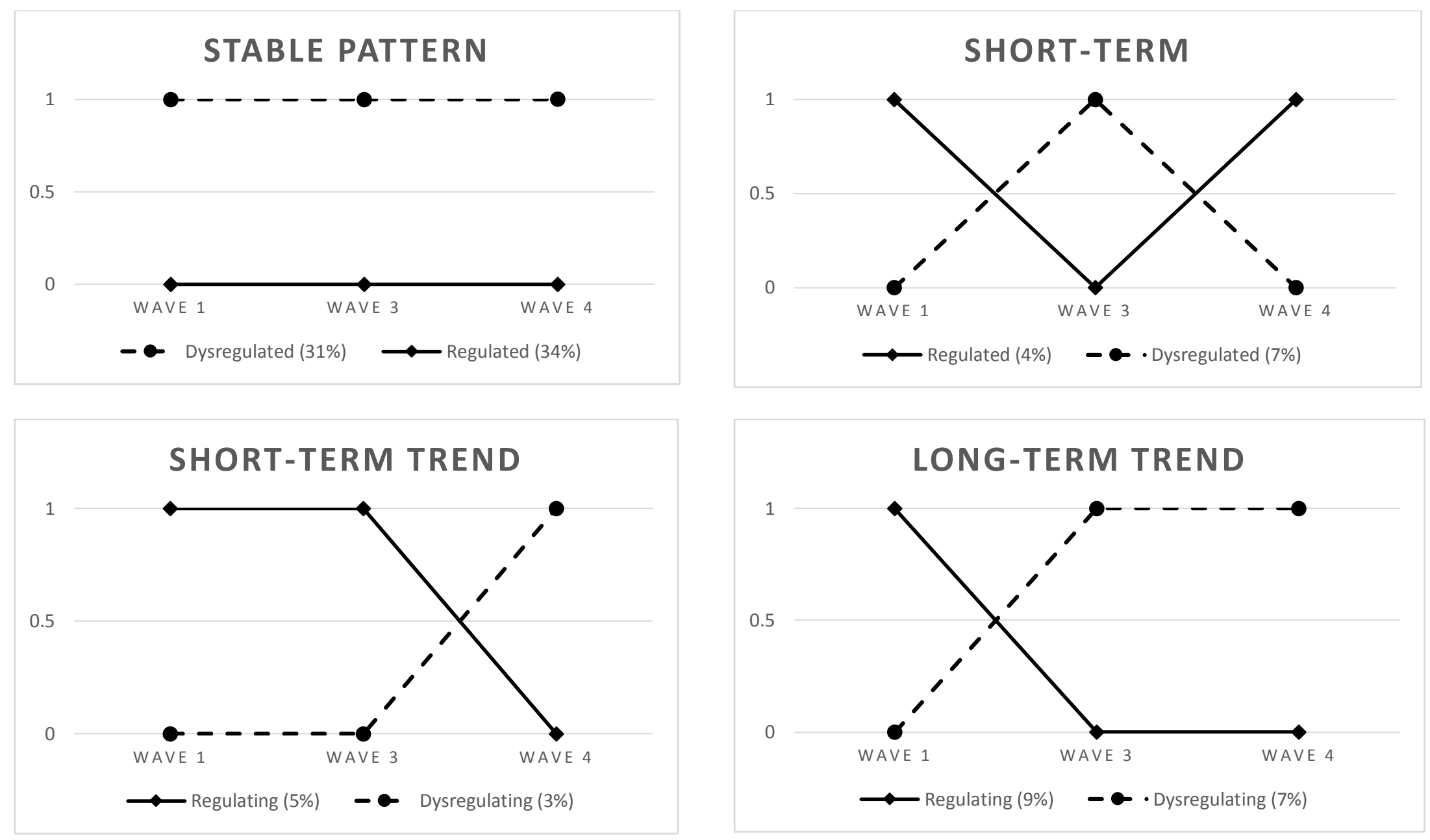

Figure 14. Developmental patterns of emotion dysregulation across waves 1, 3, and 4 


\section{Research Question 3}

The third research question aimed to examine developmental differences based on contextual factors experienced by children with a history of maltreatment. Specifically, differences in group membership was examined as a function of placement stability, caregiver emotional support, and caregiver cognitive stimulation. Gender and language were also examined as person-level factors that potentially predicted class membership. In order to answer this question, a second-order effect latent transition analysis (LTA) model was selected, building upon results from the previous section. This LTA model included time-varying transitional probabilities and covariates that were not allowed to have a time-varying effect (see Figure 15). The transitional probabilities were not constrained in order to examine how class membership transitions across the waves as a function of the selected covariates.

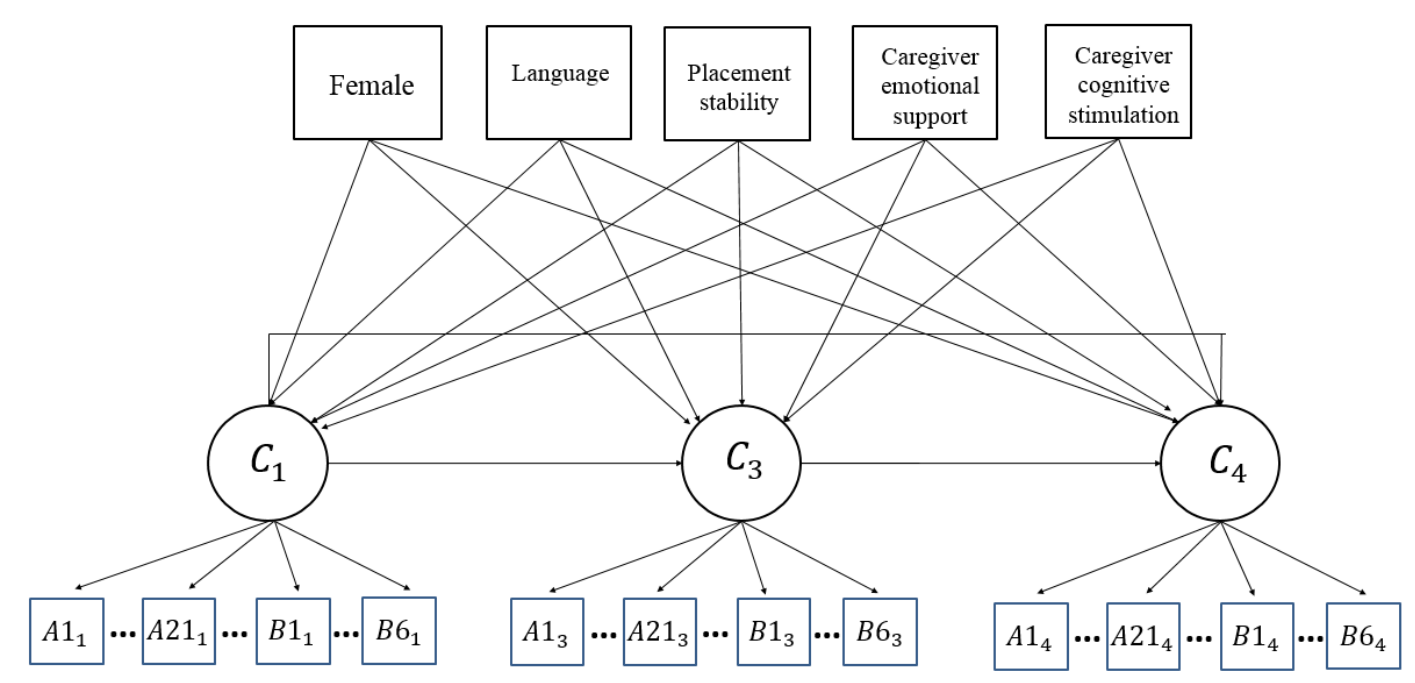

Figure 15. Second-order LTA model with covariates included 
This analysis began with the selection of a single covariate, gender, to examine transitional probabilities across the 3 waves. Gender as a categorical covariate, allowed for direct comparison of transition matrices because these are the same for boys and girls (Nylund, 2007). The resulting model had an AIC value of 74518.94, a BIC value of 73015.82, and an adjusted BIC of 74621.28. The inclusion of gender as a grouping variable improved entropy value to 0.913 compared to the unconditional model in the previous analysis, indicating good classification by the model. Table 25 shows the effect of gender on predicting class membership across the three time points. The non-significant values for gender effects across all 3 time points indicated that boys and girls were equally likely to be classified into the emotionally regulated and emotionally dysregulated classes.

Given the lack of significance in gender differences, the estimated transition probabilities for boys and girls were not reported. Table 26 displays the class counts and proportions by gender for each of the 8 response patterns. It should be noted that the classification labels for the covariate models shifted slightly compared to the unconditional models. The resulting 2 -classes were switched so that latent class 1 was the emotionally regulated (ER) latent class, while latent class 2 became the emotionally dysregulated (EdR) latent class. Given the lack of significance in the effect of gender on class assignment, this table was provided as a means to explore descriptive differences in proportion by gender. For example, the proportions for each pattern appeared to be similar across both boys and girls except for pattern 8. Boys more than girls appeared to have $5 \%$ more children who were classified as dysregulated across all three time points. 
Table 25

Odds Ratio for the Model Coefficients with Gender as a Covariate Predicting Class Membership

\begin{tabular}{cllccc}
\hline Time point & \multicolumn{1}{c}{ Class } & Gender & Coefficient & S. E. & $p$-value \\
\hline Wave 1 & Emotionally regulated & Female & -0.134 & 0.331 & 0.686 \\
Wave 3 & Emotionally regulated & Female & -0.450 & 0.460 & 0.328 \\
Wave 4 & Emotionally regulated & Female & 0.163 & 0.625 & 0.794 \\
\hline
\end{tabular}

Note. EdR was the reference class

Table 26

Class Counts and Proportions of Children within Each Latent Class by Gender $(n=834)$

\begin{tabular}{|c|c|c|c|c|c|c|c|c|c|}
\hline \multirow[t]{11}{*}{ Boys } & \multirow{2}{*}{$\begin{array}{c}\text { Latent } \\
\text { Class } \\
\text { Patterns }\end{array}$} & \multicolumn{2}{|c|}{ Wave 1} & \multicolumn{2}{|c|}{ Wave 3} & \multicolumn{2}{|c|}{ Wave 4} & \multirow[b]{2}{*}{ Class counts } & \multirow[b]{2}{*}{ Proportions } \\
\hline & & EdR & ER & EdR & ER & EdR & ER & & \\
\hline & 1 & & $\mathrm{X}$ & & $\mathrm{X}$ & & $\mathrm{X}$ & 142 & 0.171 \\
\hline & 2 & & $\mathrm{X}$ & & $\mathrm{X}$ & $\mathrm{X}$ & & 18 & 0.021 \\
\hline & 3 & & $\mathrm{X}$ & $\mathrm{X}$ & & & $\mathrm{X}$ & 33 & 0.040 \\
\hline & 4 & & $\mathrm{X}$ & $\mathrm{X}$ & & $\mathrm{X}$ & & 32 & 0.039 \\
\hline & 5 & $\mathrm{X}$ & & & $\mathrm{X}$ & & $\mathrm{X}$ & 30 & 0.036 \\
\hline & 6 & $\mathrm{X}$ & & & $\mathrm{X}$ & $\mathrm{X}$ & & 21 & 0.026 \\
\hline & 7 & $\mathrm{X}$ & & $\mathrm{X}$ & & & $\mathrm{X}$ & 28 & 0.033 \\
\hline & 8 & $X$ & & $X$ & & $\mathrm{X}$ & & 153 & 0.183 \\
\hline & & & & & & & TAL & 457 & 0.549 \\
\hline
\end{tabular}




\begin{tabular}{cccccccccc}
\hline Girls & $\begin{array}{c}\text { Latent } \\
\text { Class } \\
\text { Patterns }\end{array}$ & \multicolumn{2}{c}{ Wave 1 } & \multicolumn{2}{c}{ Wave 3 } & \multicolumn{2}{c}{ Wave 4 } & & \\
\cline { 2 - 7 } & EdR & ER & EdR & ER & EdR & ER & Class counts & Proportions \\
\hline 1 & & X & & X & & X & 143 & 0.172 \\
2 & & X & & X & X & & 5 & 0.006 \\
3 & & X & X & & & X & 26 & 0.031 \\
4 & & X & X & & X & & 24 & 0.028 \\
5 & X & & & X & & X & 44 & 0.053 \\
6 & X & & & X & X & & 12 & 0.014 \\
7 & X & & X & & & X & 16 & 0.020 \\
8 & X & & X & & X & & 107 & 0.128 \\
\hline
\end{tabular}

Note. $\mathrm{EdR}=$ Emotionally Dysregulated; ER = Emotionally Regulated 
The next step in this section included all covariates in the model. Because the additional covariates included categorical (i.e., gender and placement instability) and continuous (i.e., language wave 1, caregiver cognitive stimulation wave 1 , and caregiver emotional support wave 1), the LTA model was not specified as a multiple group analysis. Rather, all covariates were included as predictors of class membership. The resulting LTA model included a second-order effect with varying transition probabilities and time-invariant effects on the classes. The following error message was displayed in the Mplus output: "WARNING: THE BEST LOGLIKELIHOOD VALUE WAS NOT REPLICATED. THE SOLUTION MAY NOT BE TRUSTWORTHY DUE TO LOCAL MAXIMA. INCREASE THE NUMBER OF RANDOM STARTS." Based on this warning, start values were specified in the syntax as STARTS $=200$ 40, which was an increase from the initial values of STARTS $=10020$. Replication problems persisted and a start seed was included in the syntax (i.e., "STSEED = 5). This resulted in the loglikelihood being replicated. To ensure that local maxima problems did not persist, the start values were increased to STARTS $=40080$, which resulted in a successful replication.

Results indicated good model fit relative to the model that included gender as the sole covariate $(\mathrm{AIC}=60268.34, \mathrm{BIC}=60603.40$, and adjusted $\mathrm{BIC}=60368.44)$. Entropy value went down slightly compared with the one-covariate model (entropy $=0.884$ ). There were 8 response probability patterns, which was similar to the single covariate model above. Table 27 displays the class counts and proportions of these patterns based on the estimated model. 
Table 27

Class counts and proportions $(n=684)$

\begin{tabular}{crrrrrrrr}
\hline Latent Class & \multicolumn{2}{c}{ Wave 1 } & \multicolumn{2}{c}{ Wave 3 } & \multicolumn{2}{c}{ Wave 4 } & \\
Patterns & EdR & ER & EdR & ER & EdR & ER & Class counts & Proportions \\
\hline 1 & & X & & X & & X & 243 & 0.355 \\
2 & & X & & X & X & & 22 & 0.033 \\
3 & & X & X & & & X & 41 & 0.060 \\
4 & & X & X & & X & & 53 & 0.076 \\
5 & X & & & X & & X & 61 & 0.089 \\
6 & X & & & X & X & & 28 & 0.042 \\
7 & X & & X & & & X & 32 & 0.047 \\
8 & X & & X & & X & & 204 & 0.298 \\
& & & & & & TOTAL & 684 & 1.00 \\
\hline
\end{tabular}

Note . EdR $=$ Emotionally Dysregulated; $\mathrm{ER}=$ Emotionally Regulated

Results were consistent with the model above wherein covariates were not significantly related to prediction of class membership. Logistic regression coefficients are presented in Table 28. Implications for the non-significant relationship between covariates and class membership will be discussed in Chapter 5. Given that the covariates did not significantly predict class membership in this model, the next section only employed the unconditional LTA model in predicting the distal outcome of academic achievement. 
Table 28

Odds Ratio for the Model Coefficients with Covariates Predicting Class Membership

\begin{tabular}{|c|c|c|c|c|c|}
\hline Time point & Class & Covariate & Coefficient & S. E. & $p$-value \\
\hline \multirow[t]{5}{*}{ Wave 1} & Emotionally regulated & Female & 0.157 & 0.403 & 0.697 \\
\hline & & Language & 0.004 & 0.008 & 0.645 \\
\hline & & Placement instability & -0.197 & 0.381 & 0.606 \\
\hline & & Cognitive stimulation & 0.105 & 0.085 & 0.217 \\
\hline & & Emotional support & 0.100 & 0.078 & 0.200 \\
\hline \multirow[t]{5}{*}{ Wave 3} & Emotionally regulated & Female & 0.326 & 0.422 & 0.439 \\
\hline & & Language & -0.005 & 0.011 & 0.628 \\
\hline & & Placement instability & 0.240 & 0.516 & 0.642 \\
\hline & & Cognitive stimulation & 0.155 & 0.116 & 0.183 \\
\hline & & Emotional support & -0.036 & 0.100 & 0.745 \\
\hline \multirow[t]{5}{*}{ Wave 4} & Emotionally regulated & Female & 0.367 & 0.415 & 0.376 \\
\hline & & Language & 0.001 & 0.011 & 0.938 \\
\hline & & Placement instability & 0.603 & 0.539 & 0.263 \\
\hline & & Cognitive stimulation & 0.035 & 0.069 & 0.610 \\
\hline & & Emotional support & 0.071 & 0.098 & 0.470 \\
\hline
\end{tabular}

Note. EdR was the reference class; covariates were measured at wave 1 


\section{Research Question 4}

In order to answer the final research question, distal outcomes were included in the unconditional LTA model from the research question 2 analysis. Additionally, inclusion of an outcome predicted from the latent classes is an important extension of employing mixture models. These are often referred to as either a distal outcome or proximal outcomes (Muthén, 2004). These distal outcomes offer an opportunity to examine the predictive validity of the resultant latent classes. No covariates were included from the research question 3 analyses due to non-significant findings (Figure 16). The distal outcomes were collected at the $5^{\text {th }}$ wave of data collection, which ranged from 18-36 months after wave 4, or 72-90 months since the close of the Child Protective Services (CPS) investigation. The outcomes selected were the standard scores on the letter-word identification, calculation, passage comprehension, and applied problems subscales of the Woodcock-Johnson III tests of achievement. These 4 distal outcomes were directly related to the emotion dysregulation latent classes at wave 4 to examine the distal impact that emotion regulation skills may have on later achievement in school.

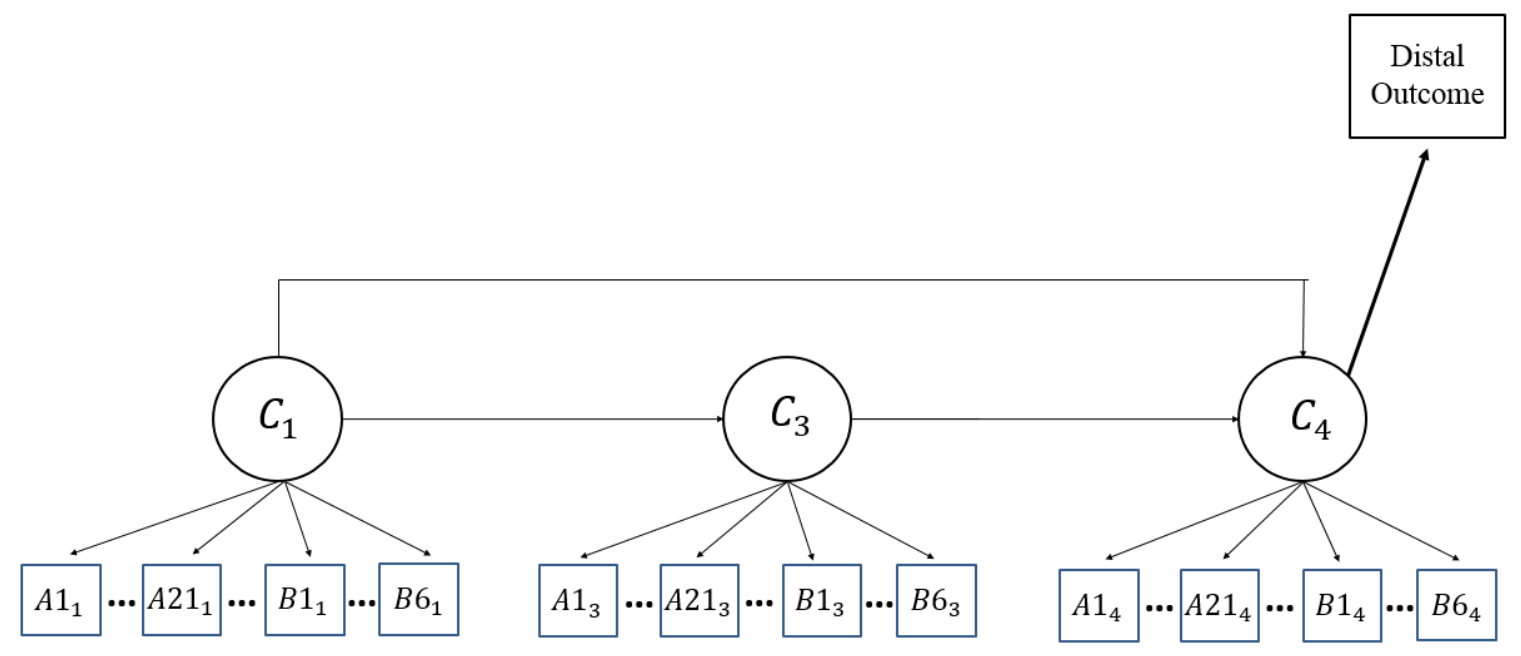

Figure 16. Second-order LTA model with distal outcome included 
Transition probabilities for the distal outcome model are presented in Table 29 alongside the unconditional LTA model. Based on the transitional probabilities of the current model, slightly less children transitioned from the EdR class at wave 1 to the ER class at wave 3 $(24.5 \%)$ when compared to the unconditional model. This pattern was repeated between wave 3 and wave 4 . There was a slight increase in the probability that children who start out dysregulated at wave 1 will remain in the same dysregulated latent class by wave 4 . Alternatively, children classified as emotionally regulated have a slightly lower probability of transitioning into the dysregulated class between waves 1 and 3 and between waves 3 and 4 .

These results indicate that dynamic processes were evident in that children transitioned between latent classes across different waves, similar to the findings from research question 2. Class counts and proportions are presented in Table 30. Similar to the unconditional model, a majority of the children fell in the stable pattern classes. That is, $34 \%$ of the children were classified as dysregulated across the 3 waves, while $32 \%$ were classified as regulated. There were 6 other latent class patterns that indicated differing classification across the waves. These patterns indicate the possibility of different pathways leading to different achievement outcomes. Table 29

Transition Probabilities for the Distal Outcome and Unconditional LTA Models $(n=834)$

\begin{tabular}{lccccc}
\hline & \multicolumn{2}{c}{ Distal outcome model } & \multicolumn{2}{c}{ Unconditional model } \\
\cline { 3 - 6 } & & \multicolumn{2}{c}{ Wave 3 } & \multicolumn{3}{c}{ Wave 3 } \\
\cline { 3 - 6 } & EdR & EdR & ER & EdR & ER \\
\hline \multirow{2}{*}{ Wave 1 } & ER & 0.755 & 0.245 & 0.740 & 0.260 \\
& & 0.253 & 0.747 & 0.270 & 0.730 \\
\cline { 3 - 6 } & EdR & Wave 4 & \multicolumn{3}{c}{ Wave 4 } \\
Wave 3 & ER & 0.773 & 0.227 & 0.755 & 0.245 \\
& & 0.157 & 0.843 & 0.132 & 0.868 \\
\hline
\end{tabular}

Note. EdR = Emotionally Dysregulated; ER = Emotionally Regulated 
Table 30

Latent Class Patterns across Waves 1, 3, and 4

\begin{tabular}{ccccccccc}
\hline & \multicolumn{9}{c}{ Wave 1 } & \multicolumn{2}{c}{ Wave 3 } & \multicolumn{2}{c}{ Wave 4 } \\
\cline { 2 - 6 } $\begin{array}{c}\text { Latent Class } \\
\text { Patterns }\end{array}$ & $\begin{array}{c}\text { EdR } \\
(1)\end{array}$ & $\begin{array}{c}\text { ER } \\
(2)\end{array}$ & $\begin{array}{c}\text { EdR } \\
(1)\end{array}$ & $\begin{array}{c}\text { ER } \\
(2)\end{array}$ & $\begin{array}{c}\text { EdR } \\
(1)\end{array}$ & $\begin{array}{c}\text { ER } \\
(2)\end{array}$ & $\begin{array}{c}\text { Class } \\
\text { counts }\end{array}$ & Proportions \\
\hline $1(111)$ & $\mathrm{X}$ & & $\mathrm{X}$ & & $\mathrm{X}$ & & 282 & 0.339 \\
$2(112)$ & $\mathrm{X}$ & & $\mathrm{X}$ & & & $\mathrm{X}$ & 51 & 0.061 \\
$3(121)$ & $\mathrm{X}$ & & & $\mathrm{X}$ & $\mathrm{X}$ & & 37 & 0.044 \\
$4(122)$ & $\mathrm{X}$ & & & $\mathrm{X}$ & & $\mathrm{X}$ & 71 & 0.085 \\
$5(211)$ & & $\mathrm{X}$ & $\mathrm{X}$ & & $\mathrm{X}$ & & 52 & 0.062 \\
$6(212)$ & & $\mathrm{X}$ & $\mathrm{X}$ & & & $\mathrm{X}$ & 47 & 0.057 \\
$7(221)$ & & $\mathrm{X}$ & & $\mathrm{X}$ & $\mathrm{X}$ & & 27 & 0.032 \\
$8(222)$ & & $\mathrm{X}$ & & $\mathrm{X}$ & & $\mathrm{X}$ & 267 & 0.320 \\
& & & & & & TOTAL & 834 & 1.00 \\
\hline
\end{tabular}

The 4 distal outcomes in the model were significantly predicted by class membership. As shown in Table 31 and Figure 17, children who were classified as emotionally dysregulated at wave 4 (54 months after the close of a CPS investigation; $M$ age $=6.56$ years, $S D=.95)$ scored lower on academic achievement than emotionally regulated children when tested at wave 5 (72 to 90 months after a CPS investigation; $M$ age $=10.21$ years, $S D=1.45$ ).

Table 31

Mean and Standard Deviation for Wave 5 Academic Achievement by Latent Class

\begin{tabular}{c|cccc}
\hline \multicolumn{5}{c}{ Wave 5 Outcomes } \\
\hline $\begin{array}{c}\text { Wave 4 } \\
\text { Classification }\end{array}$ & $\begin{array}{c}\text { WJ-Letter-word } \\
\text { ID }\end{array}$ & $\begin{array}{c}\text { WJ- } \\
\text { Calculations } \\
\text { M (SD) }\end{array}$ & $\begin{array}{c}\text { WJ-Passage } \\
\text { comprehension } \\
\text { M (SD) }\end{array}$ & $\begin{array}{c}\text { WJ-Applied } \\
\text { problems } \\
\text { M (SD) }\end{array}$ \\
\hline $\begin{array}{c}\text { Emotionally } \\
\text { dysregulated }\end{array}$ & $94.00(17.78)$ & $93.28(16.09)$ & $88.18(14.12)$ & $93.14(15.87)$ \\
$\begin{array}{c}\text { Emotionally } \\
\text { regulated }\end{array}$ & $101.97(17.78)$ & $101.74(16.09)$ & $92.57(14.12)$ & $98.65(15.87)$ \\
\hline
\end{tabular}

Note. All wave 5 distal outcomes were significant at the $p<0.001$ level. 


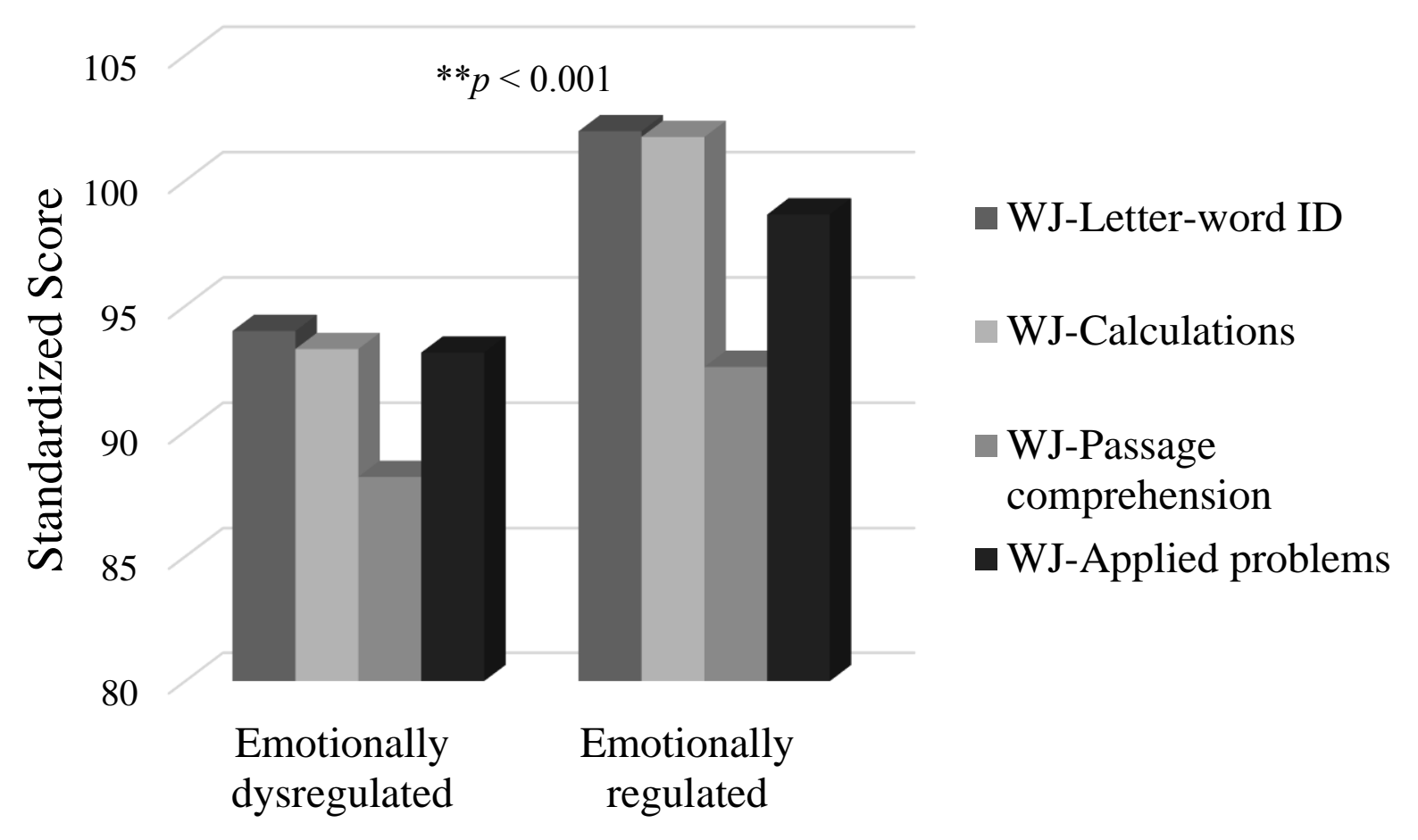

Figure 17. Academic achievement at wave 5 for emotionally regulated vs. dysregulated latent classes.

\section{Conclusion}

Based on the preceding analyses, a linear functional form for modeling growth in emotion dysregulation for the young maltreated children in this sample appeared to fit the data poorly. The measurement models for the CBCL and SSRS subscales were reliably measuring the construct of emotion dysregulation from a cross-sectional and longitudinal framework. However, when a latent growth model was fit to the data, sensitivity to growth in the parameters of interest did not appear to be captured. Although the models were specified appropriately, problems with the coarseness of the response items (i.e., 3-item Likert-type scale) and the limited number of data points made it difficult to estimate growth. Additionally, factor scores used in modeling growth need to satisfy the assumption of an underlying continuous scale. Deriving the 
continuous scores using ordinal data proved challenging with the limitations mentioned above. As such, alternative finite mixture models or person-oriented models were selected.

Subsequent analyses employed person-centered models that appeared to be better suited for categorical data in order to answer the first and second research questions. More specifically, LCA and LTA models allowed for the exploration of unobserved groups of children in this sample and how these latent classes changed over time. LCA models were first specified as measurement models to extract the number of latent classes to be used in LTA modeling. For this sample of young maltreated children, a 2-class solution appeared to fit the data well where children were classified as emotionally regulated or dysregulated. The same 2-class solution appeared to be stable across time when similar results were acquired in the cross-sectional portion of the analyses for all 3 waves.

Using LTA modeling, eight developmental patterns emerged from the data, which described how the children transitioned between the dysregulated and regulated classes across waves 1, 3, and 4. A majority of the children in this sample remained either emotionally regulated or dysregulated (34\% and $31 \%$ respectively) with the rest exhibiting one of the remaining 6 patterns. Based on these results, it is clear that clear developmental differences exist between the children in this sample. Thus, it is important to understand what influenced the children's transitional probabilities or the likelihood that they belonged to one of the developmental patterns found. This would answer the proposed third research question. Unfortunately, no significant effects were found on the covariates that were hypothesized to influence a child's emotion regulatory capacity.

Finally, research question 4 asked what pathways possibly exist that influence children's later academic achievement. Because the covariates in the previous models did not predict 
membership in either the emotion dysregulated or emotion regulated latent class, these were not included in the final model. Results indicated that a child's regulatory capacity has long-lasting effects, especially in predicting academic achievement by the early elementary years. Being classified as emotionally dysregulated by wave 3 negatively impacted later achievement scores. Given that a second-order LTA model was specified, which yielded significant results, it can be inferred that being emotionally regulated or dysregulated at wave 3 was influenced by earlier classification. Implications for these findings will be discussed in the next chapter. 


\section{Chapter 5: Discussion}

Understanding the developmental pathways to maltreated children's academic achievement is crucial for the creation of effective policies and beneficial intervention programs. Employing a bio-ecological framework, this study drew from developmental science, educational science, and child welfare perspectives to elucidate the individual- and context-level factors associated with the development of preschool-aged maltreated children and their later academic achievement. More specifically, analysis of a large-scale dataset aimed to understand the extent to which emotion regulation developed over time for a group of young maltreated children, as well as to explore whether different groups of children exhibit specific developmental patterns or classes. Additional individual-level factors (i.e., gender and language) and context-level factors (i.e., placement instability, caregiver cognitive stimulation, and caregiver emotional support) were included in order to understand how resulting proximal processes predict latent class membership (being characterized by dysregulated or regulated emotionality) for each of the children in this sample. A final goal for this study was to understand whether membership in a specific latent class during the early childhood period predicted later academic achievement for children who came into contact with Child Protective Services (CPS) as a result of suspected maltreatment.

\section{Summary and Interpretation of Results}

Achieving these goals required the application of a principled step-wise selection of models that were appropriate for understanding the development of emotion regulation/dysregulation for the sample of young maltreated children in this study. This section provides a summary of these findings beginning with specification of the measurement models. This is followed by answers to research questions 1 and 2, which asked how development of 
emotion regulation/dysregulation proceeded across time. Specific to question 2 was the inquiry about stability and change in latent classification of children's emotion regulation/dysregulation patterns. Next, individual and context-level factors that influenced development were examined in order to answer research question 3. Finally, answers to research question 4, which asked about the extent to which different developmental pathways predicted academic achievement, is presented.

\section{Measurement Models}

Results from the specification of measurement models provided evidence that items selected from the CBCL (i.e., attention, aggression, and anxiety/depression subscales) and SSRS (i.e., self-control subscale) were reliable indicators of emotion dysregulation for the 834 preschool-aged children in the sample. These results provided alternative procedures for measuring emotion dysregulation when using extant datasets that do not contain instruments specifically designed to measure emotion regulation. Indeed, the items selected for the CFA models were similar to several items from the Emotion Regulation Checklist (ERC) such as "exhibits wide mood swings," "is easily frustrated," or "prone to angry outbursts/tantrums easily" (Shields \& Cicchetti, 1997).

Additionally, results confirmed similar findings by Ayer et al. (2009). The authors conducted a series of confirmatory factor analyses that assessed CBCL post-traumatic symptom (CBCL-PTSP) and CBCL anxiety-attention-aggression (CBCL-AAA) items as measuring an underlying latent factor responsible for dysregulation in mood, behavior, and cognition. Though the database used in the current study only employed 21 of the original 118 CBCL items, the selected one-factor model replicated the same model identified in the Ayer et al. (2009) study. 
The authors hypothesized that the selected CBCL subscale items measured the single latent factor called emotion dysregulation.

The findings of the current study contributed to this literature in several ways. First, procedures for the specification of measurement models of emotion dysregulation provided an alternative way to measure emotion regulation or dysregulation when an ideal instrument is not available. Second, this investigation validated findings from the Ayer et al. (2009) study by providing similar results despite using a different analytic sample (e.g., maltreated children vs. clinical sample). Additionally, this study extended the authors' findings by including items from the self-control subscale of the SSRS, providing additional indicators for emotion dysregulation. Finally, internal consistency and construct reliability for emotion dysregulation remained high in the cross-sectional and longitudinal analyses, providing confidence in the reliability and stability of the measures.

\section{Development of Emotion Dysregulation across Time}

Research in the development of emotion dysregulation for maltreated children has been sparse. Indeed, most studies that examine emotion regulation or dysregulation in maltreated children have employed cross-sectional research designs with older children (Althoff, Rettew, Faraone, Boomsman, \& Hudziak, 2006; Althoff, Ayer, Rettew, \& Hudziak, 2010; Ayer et al., 2009). Longitudinal studies can provide better information about the dynamic nature of children's development. The identification of linear or nonlinear forms of growth can shed light on sensitive periods of development. Knowledge of these sensitive periods of development can then inform practice and policy about the timing of intervention to improve child outcomes. Latent growth models, latent class analysis models, and latent transition models were examined 
in order to understand how the development of emotion dysregulation proceeded for the children in this sample.

Latent growth models. Using the one-factor model for emotion dysregulation derived from the analyses above, a linear growth model was fit to the data to estimate growth. Results did not support the assumption that growth in emotion regulation/dysregulation for young maltreated children was continuous and linear. These results prompted a reexamination of the data structure, particularly the measurement scales of the indicator variables used in the measurement of emotion dysregulation. As a result, several models were employed to address the ordinal nature of the CBCL and SSRS response items. Methodological and empirical considerations for these findings are discussed next.

Methodological considerations. Due to poor data-model fit of the first-order latent growth models, change over time in the emotion regulation/dysregulation of young maltreated children could not be estimated in the strongest way statistically. As a solution to this problem, factor-saved scores from the measurement portion of the model were used in the analysis. This method of combining multivariate data assumed that the underlying continuous scale of the factor scores were truly captured in the model. A major problem was that the CBCL and SSRS items that loaded onto emotion dysregulation were assessed on a 3-point ordinal scale. This lack of precision in measurement may have challenged the underlying assumption of a continuous distribution (and may have caused special difficulties when measuring growth).

Thus, the next step entailed taking the ordinal nature of these items into account. Although the ordinal and dichotomized items in these second-order latent growth models fit the data well, there were no significant differences in the baseline levels and rate of growth in emotion regulation/dysregulation between the ages of 3-5 years at baseline and 6-8 years old at 
wave 4. This lack of significant growth might be due to the coarseness of the measures (i.e., lack of variability in response categories) selected to represent emotion dysregulation, as well as having a limited number of data points to model growth. The measurement models selected in the cross-sectional and longitudinal analyses appeared to fit the data well. However, when modeling growth over time, indicators with only 3 response categories are not sensitive enough to capture a wide range of responses. That is, the items selected as indicators for emotion regulation/dysregulation are not picking up the granularity of the construct given the limited response categories. When creating any form of linear combination in outcome measures, one often assumes an underlying continuous value in the factor scores. With only 3 response categories, this underlying continuous structure is often not captured.

Additionally, a growth model with only 3 data points may not provide enough room for growth in the underlying continuous factor scores within the second-order growth model. The inclusion of only three waves of data collection with large time intervals in between may not be capturing the dynamic growth in emotion regulation/dysregulation for the children in this sample. That is, sensitivity to changes in the mean levels across the growth parameters (i.e., intercept and slope) may not be captured by having only 3 time points when employing growth curve models. In this instance, application of longitudinal ANOVA models may be better suited to the investigation of changes in mean levels.

If the number of response categories and number of data points were increased, the selected model may yield significant results. In fact, a study by Long, Harring, Brekke, Test, and Greenberg (2007) employed similar procedures that resulted in significant changes in their constructs of interest. Using the Brief Symptom Inventory (BSI), the authors used nonlinear factor analysis to test graded response models of growth in the 9 subscales of the BSI. The 
authors collected data from adults diagnosed with schizophrenia every 6 months over a period of three years. Results indicated that both a fully constrained invariance model and a less constrained invariance model displayed good fit to the data. These results provided evidence that the subscale scores of the BSI were valid for studying change in these constructs over time for adults diagnosed with schizophrenia.

Given that these authors were able to model growth using ordinal data, it can be assumed that the limitation of the linear growth model for this study could be mitigated if one could use more response categories and more data points. Indeed, the models selected for this study were not incorrectly specified. Rather, data used in this study did not conform to growth, apparently because of the limitation posed by the coarseness of the response categories and the limited data points. Change over time using a latent growth model (LGM) can be seen through variations in the slope when assuming a continuous latent variable, but this was not the case in this study.

Empirical and theoretical considerations. According to Bronfenbrenner and Morris (2006), development refers to both stability and change in the characteristics of children over the life course. Understanding how development proceeds requires focusing on the features that point to more differentiated and precise theoretical formulations about change that takes into account person, context, and proximal processes. The authors propose that research conducted in an exploratory or "generative process" should examine the pattern of interdependencies within the data rather than a confirmatory/disconfirming approach. This means that an appropriate research design for testing the tenets of bio-ecological theory should involve a series of progressively more differentiated methods, with the results at each successive step informing the next. 
According to the authors, widely used models for hypothesis testing are often not appropriate for developmental investigations during exploratory procedures. This is particularly true for models that control solely for linear relationships among the variables of interest. Increased Type I errors occur due to the lack of homogeneity in these linear relationships. The authors added that violations of the homogeneity assumptions often occur with examination of developmental data. From the perspective of bio-ecological theory then, the lack of significant findings from the latent growth models may point to the need for considering alternative models that provide a better understanding of emotion regulation/dysregulation for maltreated children. From a variable-oriented perspective, the lack of variability in the mean scores across time may indicate that growth or development in emotion regulation/dysregulation did not occur. This conclusion is taken from the assumption that the children in the study were drawn from a homogenous sample. However, the assumption of group homogeneity may be incorrect, thus pointing to the inaccuracy of the resulting conclusion. In fact, when a person-centered method was employed, the heterogeneity in children's regulatory patterns became evident. Children in this sample were classified as emotionally regulated or dysregulated. Additionally, developmental patterns in how these children were classified across time emerged from the analyses.

Clear differences in the results were evident when comparing the variable-oriented and person-oriented methods. Taking into account the research questions and the data structure, development in regulatory processes were more evident when using the LCA and LTA models. These results point to the value of person-oriented models, providing additional support for the usefulness of these methods in exploring the socioemotional development of maltreated children (McWayne, Green, \& Fantuzzo, 2009; Pears, Kim, \& Fisher, 2008). 
Another possibility, of course, is that children experiencing maltreatment do not show significant growth along the regulation/dysregulation continuum. That is, organization of dysregulated emotional responses early in the maltreatment experience may have shown stability over time. In fact, maltreatment occurring between infancy and the preschool period often results in stability of maladaptive emotion regulation strategies (Maughan \& Cicchetti, 2002; Woodruff \& Lee, 2011). Given that a large number of 3-5 year olds are often returned home after a CPS investigation (Stahmer et al., 2005), these children remained in a disorganized environment. This in turn may not have provided them with opportunities to develop better adaptive emotion regulation strategies. However, given the problem with interpretability of the factor scores that represented emotion dysregulation, this conclusion may be inaccurate and warrant further attention.

Another important consideration is that the young maltreated children in this sample were recruited as a cohort from the point in time that they had contact with CPS. Though timing and prevalence of maltreatment experiences cannot be ascertained, it is reasonable to assume that first contact with CPS became a central organizer of these children's subsequent emotional regulation responses. This, in turn, may exacerbate maladaptive emotion regulatory patterns that resulted from early maltreatment (Jaffee \& Maikovich-Fong, 2011; Manly et al., 2001).

It is important to note, however, that development of emotion regulation processes start early in life (Ekas, Lickenbrock, \& Braungart-Rieker, 2013; MacLean, Rynes, Aragon, Caprihan, Phillips, \& Rowe, 2014; Martins, Soares, Martins, Tereno, \& Osorio, 2012; Ursache, Blair, Stifter, \& Voegtline, 2013). As such, emergent regulatory processes at the time of CPS involvement may be confounded by functioning and experiences during earlier development. In this case, involvement with CPS may alter the proximal processes that occur within the child's 
home due to creating a disequilibrium in the family system. According to the bio-ecological model, microtime refers to the continuity and/or discontinuity in these ongoing episodes of proximal processes (Bronfenbrenner \& Morris, 2006).

The continuity/discontinuity of proximal processes as a result of CPS involvement may then represent events wherein children organize or reorganize subsequent emotion regulation responses. Therefore, it is this organized set of responses that should be the focus of inquiry, particularly in how changes in emotion regulation/dysregulation occur across time (i.e., developmental changes). Moreover, the transactional influence of additional individual-level and context-level factors should be included. These inquiries can be answered by employing personcentered models, which are discussed in the next section.

Latent class analysis. To address the limitations of the variable-oriented models (i.e., LGMs) as a result of the limited number of time points (i.e., 3 waves of data collection) and the limited response categories (i.e., 3-point Likert scale), LCA and LTA models were employed as person-oriented alternatives to model growth and development of emotion regulation/dysregulation in the sample of young maltreated children. Results from the crosssectional LCA models provided evidence that children whose caregivers had a high probability of endorsing the items on the CBCL and SSRS subscales (i.e., lack of attention, aggression, anxiety/depression, and absence of self-control) exhibited dysregulated emotions. This provided corroborating person-centered evidence for the presence of an underlying emotion dysregulation construct responsible for problems in these areas.

Methodological considerations. Cross-sectional LCA models were specified at each of the waves (i.e., baseline, 18 months, and 36 months after a CPS investigation) to examine stability over time. By incorporating cross-sectional LCA models across 3 time points, this study 
contributed to the burgeoning literature on the application of LCA models in maltreatment research, particularly in understanding core developmental processes related to school readiness. Results of these longitudinal cross-section models indicated that the young maltreated children in this sample were consistently classified as emotionally regulated or emotionally dys regulated across time despite increasing the length of time since initial CPS contact. Bio-ecological theory proposes that later development is dependent upon earlier conditions (Bronfenbrenner and Morris, 2006). Results from this study supported this proposition.

Further, examining the longitudinal cross-sectional results indicated the possibility of changes in emotion regulation classifications across time as evidenced by shifting response probabilities for different items. For example, the item "acts too young for age" was not often endorsed in wave 1 . However, the probability of endorsing this item increased across waves 3 and 4. Changes in other item responses include, "demands a lot of attention," "fears certain animals or situations," "nervous or tense," "worries," and "temper problems with caregivers." These results are important in providing preliminary evidence that categorization of children's regulatory skills are dynamic rather than static. Furthermore, these findings provided additional evidence that LCA models, and subsequently LTA models, were appropriate for the CBCL and SSRS items using a bio-ecological framework. This in turn provided preliminary evidence that discontinuous proximal processes due to CPS involvement affects development in emotion regulation/dysregulation for the young maltreated children in this sample.

Empirical and theoretical considerations. Similar to the proposition by Ayer et al. (2009), a specific dysregulatory system may be responsible for elevated scores in the CBCL and SSRS subscales, indicating distinctly different processes between psychopathology and dysregulation. That is, a distinct core developmental process exists that may be responsible for 
problems in regulating cognitive, behavioral, and emotional processes. Additionally, these results provided support for the findings by Althoff, Ayer, Rettew, and Hudziak (2010) in which children who exhibited elevated scores on these CBCL subscales were classified as emotionally dysregulated. The authors found that a single latent class accounted for higher probabilities of endorsing these items, which signified regulatory problems in children with and without a history of trauma.

Indeed, studies that have examined developmental outcomes in maltreated children have looked at psychopathology and emotion regulation as distinctly different processes (Alink, Cicchetti, Kim, \& Rogosch, 2009; Kim-Spoon, Cicchetti, \& Rogosch, 2013; O’Mahen, Karl, Moberly, \& Fedock, 2014). Upon closer examination of the items with the highest probability of endorsement, however, they appeared to closely mirror items more related to attentional problems, internalizing behaviors, and relational aggression (e.g., can't concentrate, can't sit still, easily embarrassed, irritable, sudden changes in mood, sulks a lot, demands a lot of attention, disobedient). Higher probability of endorsement of these items may offer insight into the relationship between emotion regulation and later psychopathology in maltreated children.

For example, items that are highly correlated with internalizing behaviors may indicate an increased risk of developing anxiety or depression problems later in life. The study by O’Mahen et al. (2014), for example, provided evidence of associations between emotion regulation strategies and internalizing behaviors, and later depression in a community sample of low-income women who experienced maltreatment in early childhood. Indicators that point to relational problems could also signal problems with attachment and later psychopathology. For example, Alink et al. (2009) found evidence for the link between attachment pattern, emotion regulation, internalizing symptoms, and later psychopathology. 
The emergence of the dysregulated latent class that included both internalizing problem items and attention items was different from the findings of McWayne and Bulotsky-Shearer (2013) in their examination of developmental patterns in socioemotional school readiness. The authors found that internalizing symptoms did not generally emerge for their sample of young children in Head Start. Instead, they found higher probability of endorsing attention-related items. One possible explanation for this difference is that the authors employed a community sample of young children from Head Start while this study included young children with a history of maltreatment. If these differences can be determined to be due to maltreatment status, then the inclusion of both attention-related items and internalizing items may indicate a specific profile or latent class that distinguishes maltreated and non-maltreated children in the community. This warrants further attention.

Additional consideration must be given to possible age differences in the regulated and dysregulated classes. Results from this and the McWayne and Bulotsky-Shearer (2013) studies point to the possibility that age-salient differences can be seen in 3-, 4-, and 5-year olds. Previous studies have confirmed that regulatory strategies differ based on these ages. For example, Cole et al. (2009) found age effects for emotion regulation strategy generation where 4-year olds recognized and generated more regulatory strategies for anger, while 3- and 4-year olds recognized and generated strategies for sadness. Furthermore, these authors found that emotion regulation strategy generation was mediated by the children's expressive language skills. Further evidence of age-related distinctions in regulatory capacity was found by Kalpidou. Power, Cherry, and Gottfried (2004) where 5-, compared with 3-year olds, exhibited greater behavior and emotion regulation during a sorting task. Taken together, these studies indicate that further 
unpacking early childhood into more discrete ages may provide important distinctions on how young children's development of emotion regulation proceeds.

Latent transition analysis. In line with analytic recommendations by Bronfenbrenner and Morris (2006), results from the cross-sectional LCA analyses set the stage for examining development in emotion regulation/dysregulation using a longitudinal framework. Evidence for stability and change in young maltreated children's emotion regulation capacities were found in that the regulated and dysregulated patterns were consistent when children were 3-5 years old (i.e., wave 1, baseline), 4 1/2 - 6 1/2 years old (i.e., wave 3, 18 months after CPS investigation), and 6-8 years old (i.e., wave 4, 36 months after investigation). This suggests that structurally stable emotion regulation classes for the young maltreated children in this sample was present.

In addition, eight distinct developmental patterns emerged from the data, which showed differences in how children transitioned from one regulatory class to another across time. A majority of the children remained within their regulatory class over time. That is, over $60 \%$ of the children remained in either the regulated or dysregulated class, representing stable developmental patterns. The remainder of the sample exhibited changes in their regulatory class membership over time. These included developmental patterns that exhibited short-term (11\%), short-term trend (8\%), and long-term trend (16\%) in their transitions between regulated vs. dysregulated classes. These findings provide empirical support for the notion that development is a dynamic process rather than a static one. Further, the stability of classification for children in the emotion dysregulation class is consistent with research that underscores the stability in severely dysregulated behaviors (e.g., non-compliance, aggression) from the preschool period onward (Loeber et al., 2009; Tremblay et al., 2004; Vitaro et al., 2011). 
Differences in class proportions were evident when comparing the probability of changing emotion regulatory classes from wave 1 to 3 , and from wave 3 to 4 , where a higher number of class changes occurred during the first transition (i.e., wave 1 to 3 ) than the second transition (i.e., wave 3 to 4). One possible explanation for the higher number of class changes between wave 1 and 3 is the proximity of time since involvement of Child Protective Services (CPS). Indeed, the period between wave 1 and 3 potentially represents a tumultuous moment in a child's life when CPS intervenes. It is in this moment that an investigator is assigned to the case, which entails monitoring of the family. It is also during this moment that a child may be removed from their home depending on the severity of the maltreatment report. Additionally, severity of the maltreatment behavior may warrant home removal, which becomes another source of trauma for the child. This, in turn, negatively impacts socioemotional well-being (Wechsler-Zimring, Kearney, Kaur, \& Day, 2012). It is important to contrast this period (i.e., wave 1 to 3 ) with the transition from wave 3 to 4 , which occurred approximately 36 months after initial contact with CPS. After the initial shock in the family system, a period of 3 years post-contact with CPS may have been enough time for the family or the child to develop a sense of equilibrium in family functioning. This, in turn, resulted in fewer class transitions.

Another possible explanation for these differences in class proportions could be that between wave 1 and 3, children may be transitioning between preschool into formal schooling. Indeed, at wave 1,76\% of the children were either in preschool, Head Start, or nursery and by wave 3, 48.6\% of these children have transitioned into Kindergarten or first grade. The transition to formal schooling is a difficult time for young children who may not be "school ready" (Duncan et al., 2007; Pianta, 2007; Rouse \& Fantuzzo, 2009). Additionally, this transition to formal schooling, along with the involvement of CPS, may provide a cumulatively difficult 
experience for these young maltreated children. This, in turn, may place children at additional risk for poor academic outcomes in later grades. Examining the school transition and CPS involvement should then be considered in future research.

Stability in the child's home environment is important to consider when considering how emotionally regulated/dysregulated behaviors develop or are maintained. Maltreated children who have had contact with CPS typically have experienced one or more changes in their caregiver across time (Casanueva et al., 2014). Moreover, instability is likely to occur within the first 6 months of placement (Wulczyn, Kogan, \& Jones Harden, 2003). This places children at high risk for poor outcomes such as compromised socioemotional skills and lower academic achievement (Conger \& Finkelstein, 2003; Conger \& Rebeck, 2001; Kerbow, 1996; Rumberger \& Larson, 1998; Rumberger, 2003). Based on these results, however, it appeared that stability may not have had a consistent impact on developmental outcomes across time. That is, close proximity to the event appeared to result in increased probabilities of switching regulatory classes. These results support the notion of continuity vs. discontinuity in proximal processes within microtime, which then impact development. That is, exposure to stable or unstable interactions within the home may have differential impacts on emotion regulatory capacities. This points to the complexity of understanding the impact of the foster home and family of origin environments on children's outcomes; the quantity and quality of homes from various perspectives must be considered.

\section{Individual-level and Context-level Factors}

The inclusion of individual-level and context-level factors were examined next in order to understand what determined membership in the regulated or dysregulated class, and how these 
influenced the developmental pathway across time. The inclusion of gender, language, caregiver cognitive stimulation, and caregiver emotional support yielded non-significant results.

Methodological considerations. Several potential sources of error may be responsible for the lack of significance in these analyses. First, the selected covariates were included as timeinvariant, meaning that their effects on the transition probabilities were equal across time. Wave 1 measures were selected in order to address problems with the data structure that gave rise to missing data due to changes in measures. This potential shortcoming was addressed by employing a second-order LTA model that allowed wave 1 and wave 4 latent classes to be related. This means that development across time was allowed to be influenced by wave 1 conditions. Despite these attempts however, the selected measures taken from wave 1 may have still been problematic for predicting transitional probabilities. Second, cross-sectional validation of the LCA measurement models may need to be included. Prior to including these covariates in the LTA model, conditional LCA models could be used to conduct cross-sectional validation in order to ensure that the selected items are appropriate when deciding on the number of latent classes. Additional diagnostic procedures could be included such as differential item functioning to further validate the items used in deriving the latent classes. Third, inferring causality from the selected covariates may be problematic if possible confounders have not been accounted for. Lanza, Coffman, and Xu (2013) provide procedures for conducting causal inferencing in an LCA framework using propensity score matching and inverse propensity weighting.

Empirical and theoretical considerations. The null findings reported above were surprising given that individual-level factors such as gender have been shown to influence emotion regulation and behavior problems in young children (Denham, Bassett, Thayer, Mincic, Sirotkin, \& Zinsser, 2012; Keenan \& Shaw, 1997; Scarborough \& McCrae, 2010). However, 
non-significant gender effects have been found in other studies as well, possibly providing alternative explanations. For example, a study by Blandon, Calkins, Keane, and O'Brien (2008) found that gender did not significantly predict children's ability to regulate their emotions. According to the authors, the link between gender and regulatory processes may not be evident until adolescence when social norms around emotional expression develop further. In addition to emotion regulation measures, however, the authors included measures of negativity and found marginal gender differences were evident by age 7 wherein boys expressed higher levels of negativity compared to girls. The authors attributed the difference in negativity but not in regulation as possible divergence in regulatory processes between boys and girls. Similarly, Cole, Dennis, Smith-Simon, and Cohen (2008) conducted a study to examine emotion regulation understanding and self-regulation in preschoolers. Prior to testing the study's main models, the authors tested for gender differences in emotion understanding, which is implicated in regulatory behaviors. In their preliminary analyses, the authors did not find any gender differences in strategy understanding, leading to the combination of the data for boys and girls in the subsequent examination of emotion regulation.

Although it is possible that the measures selected for this study were not sensitive enough to capture regulatory differences between boys and girls in the preschool period, the results of these two studies suggest an alternative. It may be that gender differences in early childhood may not truly be present and that these differences could be seen at later ages. However, upon further examination of the developmental pattern differences between boys and girls, descriptive differences can be seen in that slightly higher proportions of boys belonged in the dysregulated class. As in other studies (e.g., Denham et al., 2012), these descriptive data point to the higher likelihood of emotion dysregulation among boys. 
After examination of gender as the sole predictor of class membership, the next step included all other individual-level and context-level covariates. Gender, language, placement instability, caregiver cognitive stimulation, and caregiver emotional support were examined as predictors of transition probabilities between being classified as emotionally regulated vs. dysregulated across the 3 waves. Contrary to the study hypotheses, these individual-level and context-level variables did not significantly predict developmental patterns for this sample of young maltreated children.

From a bio-ecological perspective, these context-level factors are important drivers of development (Bronfenbrenner \& Morris, 2006). That is, stability in placement provides consistent interaction over time with caregivers in order for proximal processes (i.e., cognitive stimulation and emotional support) to be promoted in the interaction of parents and children. These interactions, in turn, promote development of emotion regulation. Findings from this study, however, do not support these theoretical suppositions. There is evidence that cognitive stimulation and emotional support promote positive outcomes across developmental domains for children in the child welfare system (e.g., Jones Harden \& Whittaker, 2011). Given that maltreatment experiences represent early compromised caregiving for these children, it was important to show a distinction between maltreatment, broadly defined from these early experiences, and the dynamic influence of ongoing caregiving or parenting quality. The inclusion of the HOME inventory attempted to elucidate whether the resulting regulatory classes were due to earlier maltreatment or subsequent parenting quality. However, these distinctions were not found in the current study and need to be further investigated. The focus on transitions over time may have required a more refined examination of parenting and the home environment that took 
into account the bidirectional impact of emotion regulation and parenting and home environmental characteristics.

\section{Developmental Pathways to Academic Achievement}

The examination of the impact of emotion regulation/dysregulation development on later academic outcomes yielded significant results. Specifically, children who were classified as emotionally dysregulated by age $6 \frac{1}{2} 2$ years old (i.e., wave 4 ) scored lower on the academic achievement tests of math and reading at age 10 (i.e., wave 5). Based on earlier analyses, these regulatory patterns were stable across time. This suggests that how children regulate their emotions earlier in life can have long-term negative consequences, especially regarding their academic achievement. This is particularly important for maltreated children who come into contact with CPS during the preschool period, which is a time when children's school readiness skills become salient contributors to later school success (NRC/IOM, 2000). Emotion regulation skills are part of the broader socioemotional domain of school readiness and have been associated with school achievement (Blair, 2002; Kagan et al., 1995; Raver 2002). Thus, compromised development in emotion regulation skills impact children's school readiness broadly.

Extant research suggests that children classified as emotionally dysregulated exhibit increased problems with peer relationships (Miller et al, 2004; Sala, 2014), compromised interactions with their teachers (Graziano et al, 2006; Miller et al, 2004; Raver, 2002; Williford et al, 2013), poor classroom adjustment (Shields et al., 2001), and deficits in social competence (Denham et al., 2001). These adverse school-related outcomes were evident even after controlling for IQ (Graziano et al., 2006). Taken together, such studies underscore that emotion regulation difficulties negatively impact a child's school context, which could potentially result 
in poor academic outcomes. Based on these studies, it appears that emotion regulation is an important candidate to consider as a mechanism of change that can be the primary target of home and school interventions. In fact, according to Kagan et al. (1995), socioemotional domains are malleable and more prone to intervention effects by early childhood educators.

The negative impact of emotion dysregulation on academic achievement may also be explained by the negative effects of school transitions. Indeed, for non-maltreated children, transitioning from preschool to kindergarten results in challenges that affect school outcomes (Pianta, 2007). For maltreated children, these negative effects may be exacerbated by discontinuous proximal processes as a result of CPS involvement. For the children in this sample, the transition between wave 1 (3-5 years old) and wave 3 (i.e., 4 1/2 to $6 \frac{1 / 2}{2}$ years old) may be the point at which school entry occurs. Thus, it can be assumed that the children in this study are not only adjusting to CPS involvement, but are also adjusting to school transitions. This immediately places these children at risk for academic challenges. Stability in emotion regulatory classes may show that these risks may persist. According to Duncan and Magnuson (2011), persistence in positive or negative school readiness skills is highly predictive of later attainment.

Overall, the relation between emotion dysregulation and academic outcomes documented in the current study is consistent with the theses that emotionally dysregulated children, due to a combination of biopsychological and interpersonal processes, have more difficulty learning and have fewer opportunities for learning (Raver et al., 2007). Given the increased risk for poor academic outcomes among emotionally dysregulated children, emotion regulation should be considered a target for intervention particularly during the preschool period when children are experiencing major transitions. 


\section{Limitations}

The current study provided evidence of stability and change in the development of emotion regulation/dysregulation in young maltreated children. Furthermore, differential pathways to later academic achievement was established in that children who were classified as emotionally dysregulated at wave 4 (i.e., 6-8 years old) scored significantly lower in the math and reading achievement at wave 5 (i.e., 7 11/2 - 9 1/2 years old). Additionally, accounting for the complex sampling design ensured a nationally representative sample of young maltreated children. This means that findings can be generalized to the population of young maltreated children in the United States. However, several important limitations need to be considered.

First, this study attempted to answer questions that were longitudinal and developmental in nature and the NSCAW dataset was limited in providing the necessary data structure to answer these questions. Future research should provide enough data points to model growth. Additionally, the selected measures for emotion regulation should include more response categories when using Likert-type responses, or include additional observational measures to account for the dynamic nature of emotion regulation. For example, a study by Panlilio, Jones Harden, Harring, and Morrison (in preparation), employed an observational study design to code for children's expression and regulation of fear in a sample of young children in foster care. Using latent class growth models, the authors were able to examine 2 latent trajectory classes of fear regulation. These trajectory classes took into account the dynamic nature of emotion regulation through modeling the components (i.e., baseline, peak, and rate of increase in emotion expression) of the regulatory process. These components and the dynamic nature of emotion regulation should be considered in future studies (for a review of emotion regulation assessment tools, see Adrian, Zeman, \& Veits, 2011). 
Second, the emotion dysregulation construct relied heavily on the CBCL and SSRS items. Despite good overall model fit in the CFA and LCA frameworks, the validity of these measures have been previously questioned. For example, Fantuzzo, Manz, and McDermott (1998) investigated the reliability and validity of the teacher version of the preschool SSRS for children enrolled in Head Start. In this study, the authors ran an exploratory factor analysis to replicate the factor structure of the domains provided in the user manual. The authors found that the problem behavior domain replicated the findings presented in the manual. However, subscales in the social skills domain were not the same as the item loadings reported in the manual's factor structure. For the self-control subscale, the authors included 11 items that differed from the manual's recommended items..

In another study, Manz, Fantuzzo, and McDermott (1999) examined the psychometric properties of the parent version of the preschool SSRS in a sample of low-income, AfricanAmerican children. The authors found support for a 2-factor solution rather than a 4-factor solution for the social skills scale. Item loadings were assessed for the self-control and interpersonal skill subscales resulting in different item loadings for each of the subscale. The validity of the CBCL was examined in a study by Perry, Miller, Fusco, and Fantuzzo (2013). The authors asked Head Start parents to sort items from the CBCL based on their level of comfort in answering the questions honestly. They found 29 items that parents often categorized as provoking discomfort. Taken together, these studies point to possible problems with the validity of the CBCL and SSRS when used with an at-risk community sample of children.

Third, though the resulting latent factor was labeled as emotion dysregulation, crossvalidation of the measure was not available in this study. Given the validation issues raised by some of the studies cited above, it would be important to understand how the emotion 
dysregulation construct in this study overlaps with other measures of emotion regulation or dysregulation. One important consideration for this process is the lack of positively weighted emotion regulation items. Although the psychometric properties of the measurement models' indicator items in this study were high, and there were overlapping items with other measures like the ERC, positively weighted items were missing, for example, "can recover from stress," is warm/responsive," "is empathic" (Shields \& Cicchetti, 1997). Inclusion of these items might provide insight into resilient factors related to emotion regulation in maltreated children.

The inclusion of these additional indicators for emotion regulation will be important in capturing a complete picture of children's regulatory systems that go beyond regulation vs. dysregulation. In doing so, it may be feasible to more accurately differentiate between developmental patterns that result from other individual-level factors such as temperament from that of maltreatment experiences. For example, Zimmerman and Stansbury (2003), in a sample of 3-year olds, found that situational context and a child's temperamental reactivity were significant predictors of the regulatory strategies (i.e., comforting, distraction, cognitive, or instrumental) selected in completing a task. Others have examined the role that emotion regulation plays in mediating temperament and later social competence (Dollar \& Stifter, 2012; Eisenberg, Fabes, Guthrie, \& Reiser, 2000). Finally, other authors have argued that emotion regulation improved based on maturational processes (Kalpidou et al., 2004). These studies point to the varying perspectives with which to understand how development of emotion regulation proceeds on a continuum rather than dichotomized regulated or dysregulated classes. These need to be further evaluated in future studies.

Fourth, despite the complex sampling framework and the strength of this study's nationally representative sampling of maltreated children, caution must be exercised in extending 
these findings to the general population of young children. This study did not provide a comparative community sample that would allow for generalization of results beyond maltreated children.

Another limitation is that the predominant reason for referral in this sample was neglect. There may be specific subgroup differences that could not be assessed (e.g. sexual abuse in particular). Another limitation is the absence of information about siblings (both in the family of origin and the foster family). For example, Pears, Kim, and Fisher (2008) employed the Maltreatment Classification System (MCS) to code for different maltreatment types and found that specific profiles of maltreatment experiences were related to differences in cognitive functioning, externalizing problems, and internalizing problems. Manly, Kim, Rogosch, and Cicchetti (2001) also found similar results where developmental outcomes differed by maltreatment type. These studies point to the need that further analysis employing maltreatment subtypes are warranted.

Finally, measures of proximal processes between the caregiver and child using the HOME may not have accurately captured the dynamic nature of the family system. The HOME inventory, which includes the cognitive stimulation and emotional support subscales, describes main areas of a child's caregiving environment based on the presence or absence of selected caregiving indicators. These are often conducted at one point in time during the interviewer's visits. The transactional nature of the parent-child relationship responsible for developmental outcomes may not be accurately captured by this instrument. These proximal processes that need to occur consistently over time need to be better captured in order to directly relate developmental outcomes of interest such as emotion regulation. The binary response categories for the HOME inventory may also be limited in capturing frequency or severity of the targeted 
item. For example, if the question asks about the occurrence of physical punishment, a score of 1 for yes or 0 for no does not accurately capture how often this occurred in the home. Limitations of the HOME inventory have also been presented by Totsika and Sylva (2004) in their review of the measures clinical utility and psychometric properties.

\section{Future Research}

In addition to the limitations and suggested future directions discussed in earlier sections, this section outlines additional recommendations for future research. Bio-ecological theory proposes that developmental changes occur as a result of stable and consistent interaction with a child's environment. The dynamic nature of this proposition was not captured in this study. As such, future research should consider employing alternate measures or research designs that would allow for this. For example, observational studies allow for measuring immediate parentchild interactions. Coding procedures could be adopted or created in order to code for parenting behaviors that relate to emotion regulation development. These could include a more refined coding system that examines parental responsiveness to children's presentation of emotionally dysregulated behaviors in the moment and specific strategies in the family/home environment to support regulation (e.g., quiet times) might allow for more valid evidence in this regard.

Extending the latent transition analysis to include parent behavior measures may also improve upon this study. This is similar to the procedures employed by McWayne and BulotskyShearer (2013) in their LTA models. Inclusion of covariates within their latent class models yielded significant results in the prediction of class membership and transitional probabilities in a sample of young children in Head Start. Alternatively, increasing the number of measures on parenting behavior allow for its inclusion as a time-varying covariate. In doing so, analytic models are allowed to model changes in growth that takes into account the covariates' influence 
in their respective time point. Given the movements that occur in children's placements when CPS gets involved, these procedures may closely match the experiences of maltreated children.

Next, proximal process variables in this study were limited to the home setting. When children transition from preschool to Kindergarten and beyond, the school microsystem is added into their expanding context. Thus, interest in later academic achievement should include schoolrelated factors such as measures of classroom quality (i.e., instructional quality, emotional, and organizational support), transition to formal schooling, and school mobility. In addition to classroom-level factors, parent involvement in school should be considered. For maltreated children in particular, these factors may be affected by their involvement with CPS. When considering data analysis, future research should consider moving beyond a multi-trait-multiinformant method to examine these multilevel variables' impact on child outcomes. Rather, more person-centered approaches that consider the whole child should be employed. Doing so places methodologies in line with developmental theories of change.

Another consideration for future research is the inclusion of other covariates that may influence emotion regulation development and later school achievement. For example, maltreatment type and other maltreatment risk factors (e.g., poverty) are candidates for empirical examination. Inclusion of these covariates could provide better information about sources of variability in the developmental outcomes of young maltreated children. Other factors to consider in future research include different child care or school settings that might affect later school functioning. Indeed, the sample in this study at baseline included a majority of children who were not in any formal school or care setting, while the next largest group of children were enrolled in Head Start. Future studies must consider the potential impact that these early care settings may have on school readiness and later school achievement. 
Finally, future research should incorporate multiple measures of emotion regulation. These measures should include more than 3 response categories. In fact, larger response categories (e.g., 7-point Likert scale) will provide better replication of an underlying latent continuum in studies that will employ latent growth and growth mixture models. . Observational measures of emotion regulation, as opposed to the caregiver report measures in the current study, could also enrich investigations in this domain. Additionally, measurement occasions should be increased beyond three time points in order for the models to detect significant changes in mean levels across time. This also allows for inclusion of other growth parameters to model multiple forms of growth (e.g., quadratic, cubic). Inclusion of multiple emotion regulation measures can provide the researcher with an opportunity to cross-validate measurement models that examine emotion regulation as a latent factor.

Future research that are based on better measures and improved research design can help further our understanding of the link between maltreatment, school readiness, and later academic outcomes. More importantly, guiding theory, along with empirical evidence for the causal relationships leading to these compromised school outcomes can inform prevention programs by providing empirically-derived mechanisms for change (for a detailed discussion on causal links in program outcomes, see Lanier, Maguire-Jack, Mienko, \& Panlilio, 2015).

\section{Implications}

Experiencing maltreatment and CPS involvement negatively impact children's emotion regulation development. Emotion regulation skills have been implicated as a necessary foundation for early school success (Blair, 2002; Raver, 2002; Raver, Garner, \& Smith-Donald, 2007). When these adverse events happen, particularly during early childhood, developmental outcomes are negatively affected. 
This study contributed to the literature by showing the stability and change in maltreated children's emotion regulation patterns across time. In addition, significant findings relating emotion regulation to later academic achievement have important implications for intervention and policy in addressing the academic vulnerabilities of young maltreated children. These are discussed next.

\section{Theory}

Bio-ecological theory provided an important framework with which to examine the developmental processes and academic outcomes of young maltreated children. Changes in emotion regulation/dysregulation classes over time supported the notion that person characteristics are important for producing stability and change (i.e., development) in socioemotional functioning. More specifically, person or individual-level factors are important to understand because certain traits or characteristics of a person (e.g., emotional disturbance, behavior problems, or learning disability) may serve as risk factors for ongoing maltreatment and poor academic outcomes.

Additionally, this study provided support for the effect of discontinuity in episodes of proximal processes within the microtime level. This was evident in the different rates of emotion regulation class transitions based on proximity to the initial contact with CPS. Finally, partial support for the theory's proposition that the effects of proximal processes vary based on the developmental outcome. That is, developmental impact of proximal processes for children in disorganized environments will occur mainly for developmental dysfunction while for children in stable environments, proximal processes will have a greater impact on competence (Bronfenbrenner \& Morris, 2006). This notion that different developmental pathways lead to different outcomes was evident in the emergence of different developmental pathways based on 
children's regulatory pattern, as well as the significant relationship in predicting later academic achievement.

Unfortunately, the discontinuity of proximal processes that lead to different regulatory classes could only be assumed. The inclusion of caregiver emotional support and cognitive stimulation did not yield significant results. However, Bronfenbrenner and Morris (2006) stipulated that the aim of exploratory research to test theoretical propositions was not to claim empirical validity but to indicate inclusion in research design at subsequent stages of exploratory work. That said, these non-significant findings provided important theoretical implications by validating a point made earlier in this chapter. That is, interest in developmental research means that the primary focus should be on developing an iterative process for research designs. Nonsignificant findings from this study may signify that the methodology was limited in its ability to distinguish proximal processes from context. Thus, the suggestions in the earlier sections could provide more precise patterns of relationships among the variables and improved research design for future research.

\section{Intervention}

The notion that not all maltreated children develop in a uniform manner should be taken into consideration when designing intervention programs for improving academic outcomes. Given the importance of socioemotional development as a school readiness indicator, it is necessary to consider core developmental processes such as emotion regulation when designing interventions, particularly in addressing the school vulnerabilities of maltreated children. Results from this study support the proposition that emotion regulation, an important component of the socioemotional domain of school readiness, is impacted by early experiences of maltreatment 
and involvement of CPS. More importantly, emotion regulation can provide a mechanism with which to address later academic achievement.

The long-term impact of maltreatment and CPS involvement is salient for the children in this study. This is especially important given the negative impact of dysregulated emotions on achievement even at later ages. As such, the timing, type, and dosage of services can be altered based on the developmental needs of children with a history of maltreatment. By adopting a developmental perspective in the creation and delivery of intervention services, the field can move away from a deficit approach.

To this end, early childhood educators will need to know how to adapt educational practices based on the circumstances that a child comes into the school with. For example, early assessment of young children's socioemotional functioning should occur prior to school entry in order to identify these developmental needs for children. Additional contextual factors should be included in order to identify potential environmental stressors that may tax a child's regulatory capacity, especially in the period when a child transitions into formal schooling. The identification of these additional risk factors could help educators and other providers to initiate the appropriate type and dosage of intervention services that are based on targeted needs. Early childhood educators should be able to help young maltreated children in their classroom emply appropriate emotional regulation strategies. These in turn will help young maltreated children with the socioemotional demands in the classroom with teachers and peers, identify emerging emotions elicited by these socioemotional demands, and learn how to modulate emotional responses during emotion-eliciting events or activities.

Intervention programs should also consider the whole child that takes into account the school microsystem, the home microsystem, and the Child Welfare microsystem. For example, 
when designing interventions to mitigate academic risk and vulnerability, core developmental processes from the individual level should be assessed. Specifically, for children in the emotionally dysregulated category, early interventions can facilitate caregiver support of children's strategies to deploy their cognitive and emotional resources to modulate themselves (e.g., mindfulness, problem-solving). Developmental considerations in designing intervention programs should also take into account the multiple environments in which a child grows. For example, the microsystems in the child's life should be brought together (e.g., school, home, foster home, and CPS) in interventions, as a way to ensure that the child is getting similar types and quality of support across these multiple environments to promote emotion regulation.

Based on the recommendations provided above, adoption of a Head Start program model that includes additional services for children with a history of maltreatment may be important to consider. Nationally, Head Start promotes the school readiness of young children by providing comprehensive services that address the education needs of young children at risk. In addition to the education needs of young children, these comprehensive services include developmental screening, social and emotional health, social services, and family services among others. As of 2014, Head Start programs served 17,858 children in foster care (U. S. Department of Health and Human Services, Administration for Children and Families, 2014). However, based on the sample in this study, a nationally representative number of children involved with CPS are not in long-term foster care. Additionally, the evidence that emotion regulation was compromised for this sample of children indicate that Head Start services should take into account children's maltreatment histories in order to help promote school readiness. Family services should also account for the different microsystems that children in this sample may experience (e.g., family of origin, out-of-home placement, schools). 
In order to address the academic needs of children above 5 years of age, a similar service model to Head Start should be implemented by school systems. Continuation of the comprehensive services that were available during the Head Start period should be able to help with the transition to formal schooling. In addition, teachers at the primary grades could receive trauma-informed training in order to adapt educational practices for these children transitioning from preschool. Thus, a continuum of care that promotes emotion regulation development within the school, home, and CPS contexts could potentially mitigate the academic problems at later ages.

\section{Policy}

Children with a history of maltreatment are involved in a complex, multi-systemic context that have impact their developmental processes. This is particularly salient for the development of school readiness in the preschool period. It is therefore imperative that education and child welfare systems work in tandem to help promote positive change for children with a maltreatment history. Currently, the CAPTA Reauthorization Act of 2010 provides recommendations for children's access to an Individualized Family Services Plan (IFSP) under the Individuals with Disabilities Education Act (IDEA, 2004), as well as access to Part C of the IDEA, which authorizes states to access funds for early intervention services.

The IDEA was reauthorized to be aligned with the No Child Left Behind (NCLB, 2003) Act, which became effective on July 1, 2005. Under this realignment, certain regulations may make it more feasible to provide better services for maltreated children. For example, "scientifically based research" has been added into the IDEA, which attempts to ensure that interventions that are put in place, meet the minimal definitions provided. Additionally, the section that requires states to link records for migratory children could allow school districts to 
more readily share academic records for maltreated children with multiple placements. Finally, there is a section that requires additional qualifications and training for special education teachers. Better understanding of teaching practices that work for maltreated children could provide information on teacher qualifications to teach these vulnerable students.

In addition to the education-related policies stated above, federal policies from the Office of Head Start indicate that there are regional priorities to collaborate with Child Welfare. Currently, Early Head Start agency partnerships with Child Welfare attempt to address the developmental needs of children aged 0 to $3^{1}$. Additionally, service arrays from The Children's Bureau at the Administration for Children and Families currently offer education-related services to children who are in out-of-home care ${ }^{2}$. Though commendable for these current policies, results from this study indicated that children between the ages of 3 and 5, who have a history of maltreatment, and who remain in their home, exhibited compromised emotion regulation skills. In addition, problems with emotion regulation early in life have negative consequences on these children's academic achievement in later school grades. Thus, current policies should take these findings into account by extending the Head Start-Child Welfare partnership beyond the 0-3 age range, moving the Child Welfare collaboration priority from regional to the national level, and for the Children's Bureau to extend educational services to all children who come into contact with CPS and not just for children in foster care.

\section{Conclusion}

Results from this study showed that young maltreated children who came into contact with CPS during early childhood were classified as being emotionally regulated or dysregulated across time. In addition, children who were classified as being emotionally dysregulated by age

\footnotetext{
${ }^{1}$ Source: http://eclkc.ohs.acf.hhs.gov/hslc/states/collaboration/about.html

${ }^{2}$ Source: https://www.childwelfare.gov/topics/systemwide/service-array/education-services/
} 
$6 \frac{1}{2}$ (i.e., wave 4 of data collection) scored significantly lower than emotionally regulated children in reading and math achievement at age 10 (i.e., wave 5 of data collection). Initial results from latent growth models did not yield results in the hypothesized direction. However, application of person-oriented models provided information about patterns in young maltreated children's emotion regulation/dysregulation. These results point to the value of applying personoriented models in understanding growth and development in emotion regulation of young maltreated children. These patterns appeared to exhibit stability and change in emotion regulation/dysregulation, hallmark definitions of development. Developmental patterns that emerged indicated that some children transitioned between being emotionally regulated and dysregulated, which provided evidence of the dynamic nature of developmental processes. This warranted further investigation on the determinants of class membership in these regulatory patterns. Based on bio-ecological theory, caregiver emotional support and cognitive stimulation were examined, along with gender and language, to understand how each might influence membership in the dysregulated or regulated emotional classes and how these class membership changed over time. Though inclusion of person-level and context-level factors yielded nonsignificant results, it is clear that further examination of the dynamic nature of these covariates are needed based on bio-ecological theory's proposition that continuity of proximal processes are important drivers of development. Finally, compromised math and reading achievement as a result of belonging to the emotionally dysregulated latent class points to the important role that socioemotional development plays in making children more socially and emotionally ready for school. Thus, educators and CPS workers should consider emotion regulation development as an important mechanism for change when attempting to improve later school outcomes of young maltreated children. 


\section{Appendix A: Sampling Domains for the NSCAW CPS Sample}

Table A1

Within-PSU Sampling Domains for the NSCAW CPS Sample

\begin{tabular}{|c|c|}
\hline Domain & Description \\
\hline 1 & Infants (age $<1$ year old) who were not receiving CPS agency funded services \\
\hline 2 & Children age 1 to 14 years old who were not receiving CPS agency funded services \\
\hline 3 & $\begin{array}{l}\text { Infants (age }<1 \text { year old) who were receiving CPS agency funded services and were } \\
\text { not in out of home care }\end{array}$ \\
\hline 4 & $\begin{array}{l}\text { Children age } 1 \text { to } 14 \text { years old who were receiving CPS agency funded services, were } \\
\text { not in out of home care, and were investigated for allegations of sexual abuse }\end{array}$ \\
\hline 5 & $\begin{array}{l}\text { Children age } 1 \text { to } 14 \text { years old who were receiving CPS agency funded services, were } \\
\text { not in out of home care, and were investigated for allegations of other abuse or neglect }\end{array}$ \\
\hline 6 & $\begin{array}{l}\text { Infants (age }<1 \text { year old) who were receiving CPS agency funded services and were in } \\
\text { out of home care }\end{array}$ \\
\hline 7 & $\begin{array}{l}\text { Children age } 1 \text { to } 14 \text { years old who were receiving CPS agency funded services, were } \\
\text { in out of home care, and were investigated for allegations of sexual abuse }\end{array}$ \\
\hline 8 & $\begin{array}{l}\text { Children age } 1 \text { to } 14 \text { years old who were receiving CPS agency funded services, were } \\
\text { in out of home care, and were investigated for allegations other abuse or neglect }\end{array}$ \\
\hline
\end{tabular}

Source: NSCAW Data File User's Manual (DFUM; 2008) 


\section{Appendix B: NSCAW Sampling Weights}

Table B1

Available Weights for Analyzing NSCAW Data

\begin{tabular}{ll}
\hline $\begin{array}{c}\text { Weighting } \\
\text { Variable Name }\end{array}$ & \multicolumn{1}{c}{ Description } \\
\hline NANALWT & Cross-sectional weight for national-level estimates, Wave 1 \\
SANALWT & Cross-sectional weight for stratum-level estimates, Wave 1 \\
NANALWT2 & Longitudinal weight for national-level estimates, Wave 2, or Waves 1 and 2 \\
SANALWT2 & Longitudinal weight for stratum-level estimates, Wave 2, or Waves 1 and 2 \\
NANALWT3 & Longitudinal weight for national-level estimates, Wave 3, or Waves 1 and 3 \\
SANALWT3 & Longitudinal weight for stratum-level estimates, Wave 3, or Waves 1 and 3 \\
NANALWT4 & Longitudinal weight for national-level estimates, Wave 4, or Waves 1 and 4 \\
SANALWT4 & Longitudinal weight for stratum-level estimates, Wave 4, or Waves 1 and 4 \\
NANALWT34 & Longitudinal weight for national-level estimates, Waves 1, 3, and 4 \\
SANALWT34 & Longitudinal weight for stratum-level estimates, Waves 1, 3, and 4 \\
NANALWT5 & Longitudinal weight for national-level estimates, Wave 5, or Waves 1 and 5 \\
NANALWT345 & Longitudinal weight for national-level estimates, Waves 1, 3, 4, and 5 \\
\hline SOHCe: NSCAW Statistical User's Matimat (2008)
\end{tabular}

Source: NSCAW Statistical User's Manual (2008) 


\section{Appendix C: HHS Poverty Guidelines}

Table C1

\begin{tabular}{cccc}
\hline \multicolumn{3}{c}{$\mathbf{2 0 0 0}$} \\
\hline $\begin{array}{c}\text { Size of Family } \\
\text { Unit }\end{array}$ & $\begin{array}{c}\text { 48 Contiguous } \\
\text { States and D.C. }\end{array}$ & Alaska & Hawaii \\
\hline $\mathbf{1}$ & $\$ 8,350$ & $\$ 10,430$ & $\$ 9,590$ \\
$\mathbf{2}$ & $\$ 11,250$ & $\$ 14,060$ & $\$ 12,930$ \\
$\mathbf{3}$ & $\$ 14,150$ & $\$ 17,690$ & $\$ 16,270$ \\
$\mathbf{4}$ & $\$ 17,050$ & $\$ 21,320$ & $\$ 19,610$ \\
$\mathbf{5}$ & $\$ 19,950$ & $\$ 24,950$ & $\$ 22,950$ \\
$\mathbf{6}$ & $\$ 22,850$ & $\$ 28,580$ & $\$ 26,290$ \\
$\mathbf{7}$ & $\$ 25,750$ & $\$ 32,210$ & $\$ 29,630$ \\
$\mathbf{8}$ & $\$ 28,650$ & $\$ 35,840$ & $\$ 32,970$ \\
For each & & & \\
additional person, & $\$ 2,900$ & $\$ 3,630$ & $\$ 3,340$ \\
add & & & \\
\hline
\end{tabular}

Source: Federal Register, Vol. 65, No. 31, February 15, 2000, pp. 7555-7557

Table C2

\section{1}

\begin{tabular}{cccc}
\hline $\begin{array}{c}\text { Size of Family } \\
\text { Unit }\end{array}$ & $\begin{array}{c}\text { 48 Contiguous } \\
\text { States and D.C. }\end{array}$ & Alaska & Hawaii \\
\hline $\mathbf{1}$ & $\$ 8,590$ & $\$ 10,730$ & $\$ 9,890$ \\
$\mathbf{2}$ & $\$ 11,610$ & $\$ 14,510$ & $\$ 13,360$ \\
$\mathbf{3}$ & $\$ 14,630$ & $\$ 18,290$ & $\$ 16,830$ \\
$\mathbf{4}$ & $\$ 17,650$ & $\$ 22,070$ & $\$ 20,300$ \\
$\mathbf{5}$ & $\$ 20,670$ & $\$ 25,850$ & $\$ 23,770$ \\
$\mathbf{6}$ & $\$ 23,670$ & $\$ 29,630$ & $\$ 27,240$ \\
$\mathbf{7}$ & $\$ 26,710$ & $\$ 33,410$ & $\$ 30,710$ \\
$\mathbf{8}$ & $\$ 29,730$ & $\$ 37,190$ & $\$ 34,180$ \\
For each & & & \\
additional person, & & & $\$ 3,470$ \\
add & $\$ 3,020$ & $\$ 3,780$ & \\
\hline
\end{tabular}

Source: Federal Register, Vol. 66, No. 33, February 16, 2001, pp. 10695-10697 
Table C3

\begin{tabular}{cccc}
\hline \multicolumn{4}{c}{$\mathbf{2 0 0 2}$} \\
\hline $\begin{array}{c}\text { Size of Family } \\
\text { Unit }\end{array}$ & $\begin{array}{c}\text { 48 Contiguous } \\
\text { States and D.C. }\end{array}$ & Alaska & Hawaii \\
\hline $\mathbf{1}$ & $\$ 8,860$ & $\$ 11,080$ & $\$ 10,200$ \\
$\mathbf{2}$ & $\$ 11,940$ & $\$ 14,930$ & $\$ 13,740$ \\
$\mathbf{3}$ & $\$ 15,020$ & $\$ 18,780$ & $\$ 17,280$ \\
$\mathbf{4}$ & $\$ 18,100$ & $\$ 22,630$ & $\$ 20,820$ \\
$\mathbf{5}$ & $\$ 21,180$ & $\$ 26,480$ & $\$ 24,360$ \\
$\mathbf{6}$ & $\$ 24,260$ & $\$ 30,330$ & $\$ 27,900$ \\
$\mathbf{7}$ & $\$ 27,340$ & $\$ 34,180$ & $\$ 31,440$ \\
$\mathbf{8}$ & $\$ 30,420$ & $\$ 38,030$ & $\$ 34,980$ \\
For each & & & \\
additional person, & $\$ 3,080$ & $\$ 3,850$ & $\$ 3,540$ \\
add & & & \\
\hline
\end{tabular}

Source: Federal Register, Vol. 67, No. 31, February 14, 2002, pp. 6931-6933 
Appendix D: Cross-tabulation for Poverty Estimation

Table D1

\begin{tabular}{|c|c|c|c|c|c|c|c|c|c|c|c|c|c|c|c|}
\hline \multirow{2}{*}{\multicolumn{2}{|c|}{ Income level }} & \multicolumn{13}{|c|}{ Number in household: Wave 1} & \multirow[b]{2}{*}{ Total } \\
\hline & & 0 & 1 & 2 & 3 & 4 & 5 & 6 & 7 & 8 & 9 & 10 & 13 & 17 & \\
\hline \multirow{2}{*}{$\begin{array}{l}\text { PARTIAL } \\
\text { INTV }\end{array}$} & Count & 0 & 0 & 0 & 0 & 0 & 0 & 0 & 0 & 0 & 0 & 0 & 0 & 0 & 3 \\
\hline & $\begin{array}{l}\% \text { of } \\
\text { Total }\end{array}$ & $\begin{array}{r}0.0 \\
\%\end{array}$ & $\begin{array}{r}0.0 \\
\%\end{array}$ & $0.0 \%$ & $0.0 \%$ & $0.0 \%$ & $0.0 \%$ & $0.0 \%$ & $\begin{array}{r}0.0 \\
\%\end{array}$ & $\begin{array}{r}0.0 \\
\%\end{array}$ & $\begin{array}{r}0.0 \\
\%\end{array}$ & $\begin{array}{r}0.0 \\
\%\end{array}$ & $\begin{array}{r}0.0 \\
\%\end{array}$ & $\begin{array}{r}0.0 \\
\%\end{array}$ & $.4 \%$ \\
\hline \multirow{2}{*}{$\begin{array}{l}\text { REFUSE } \\
\text { D }\end{array}$} & Count & 0 & 0 & 0 & 0 & 0 & 0 & 0 & 0 & 0 & 0 & 0 & 0 & 0 & 39 \\
\hline & $\begin{array}{l}\% \text { of } \\
\text { Total }\end{array}$ & $\begin{array}{r}0.0 \\
\%\end{array}$ & $\begin{array}{r}0.0 \\
\%\end{array}$ & $0.0 \%$ & $0.0 \%$ & $0.0 \%$ & $0.0 \%$ & $0.0 \%$ & $\begin{array}{r}0.0 \\
\%\end{array}$ & $\begin{array}{r}0.0 \\
\%\end{array}$ & $\begin{array}{r}0.0 \\
\%\end{array}$ & $\begin{array}{r}0.0 \\
\%\end{array}$ & $\begin{array}{r}0.0 \\
\%\end{array}$ & $\begin{array}{r}0.0 \\
\%\end{array}$ & $4.7 \%$ \\
\hline \multirow{2}{*}{$\begin{array}{l}\text { DONT } \\
\text { KNOW }\end{array}$} & Count & 1 & 0 & 1 & 5 & 8 & 1 & 6 & 1 & 1 & 0 & 0 & 0 & 0 & 32 \\
\hline & $\begin{array}{l}\% \text { of } \\
\text { Total }\end{array}$ & $.1 \%$ & $\begin{array}{r}0.0 \\
\%\end{array}$ & $.1 \%$ & $.6 \%$ & $1.0 \%$ & $.1 \%$ & $.7 \%$ & $.1 \%$ & $.1 \%$ & $\begin{array}{r}0.0 \\
\%\end{array}$ & $\begin{array}{r}0.0 \\
\%\end{array}$ & $\begin{array}{r}0.0 \\
\%\end{array}$ & $\begin{array}{r}0.0 \\
\%\end{array}$ & $3.8 \%$ \\
\hline \multirow[t]{2}{*}{$<5000$} & Count & 0 & 2 & 17 & 15 & 16 & 6 & 0 & 0 & 1 & 0 & 0 & 1 & 0 & 58 \\
\hline & $\begin{array}{l}\% \text { of } \\
\text { Total }\end{array}$ & $\begin{array}{r}0.0 \\
\%\end{array}$ & $.2 \%$ & $2.0 \%$ & $1.8 \%$ & $1.9 \%$ & $.7 \%$ & $0.0 \%$ & $\begin{array}{r}0.0 \\
\%\end{array}$ & $.1 \%$ & $\begin{array}{r}0.0 \\
\%\end{array}$ & $\begin{array}{r}0.0 \\
\%\end{array}$ & $.1 \%$ & $\begin{array}{r}0.0 \\
\%\end{array}$ & $7.0 \%$ \\
\hline \multirow[t]{2}{*}{ 5K-9999 } & Count & 0 & 1 & 28 & 39 & 30 & 19 & 11 & 2 & 1 & 1 & 0 & 0 & 0 & 133 \\
\hline & $\begin{array}{l}\% \text { of } \\
\text { Total }\end{array}$ & $\begin{array}{r}0.0 \\
\%\end{array}$ & $.1 \%$ & $3.4 \%$ & $4.7 \%$ & $3.6 \%$ & $2.3 \%$ & $1.3 \%$ & $.2 \%$ & $.1 \%$ & $.1 \%$ & $\begin{array}{r}0.0 \\
\%\end{array}$ & $\begin{array}{r}0.0 \\
\%\end{array}$ & $\begin{array}{r}0.0 \\
\%\end{array}$ & $15.9 \%$ \\
\hline \multirow{2}{*}{$\begin{array}{l}10 K- \\
14999\end{array}$} & Count & 0 & 2 & 17 & 24 & 35 & 23 & 13 & 4 & 3 & 0 & 0 & 0 & 0 & 121 \\
\hline & $\begin{array}{l}\% \text { of } \\
\text { Total }\end{array}$ & $\begin{array}{r}0.0 \\
\%\end{array}$ & $.2 \%$ & $2.0 \%$ & $2.9 \%$ & $4.2 \%$ & $2.8 \%$ & $1.6 \%$ & $.5 \%$ & $.4 \%$ & $\begin{array}{r}0.0 \\
\%\end{array}$ & $\begin{array}{r}0.0 \\
\%\end{array}$ & $\begin{array}{r}0.0 \\
\%\end{array}$ & $\begin{array}{r}0.0 \\
\%\end{array}$ & $14.5 \%$ \\
\hline \multirow{2}{*}{$\begin{array}{l}15 \mathrm{~K}- \\
19999\end{array}$} & Count & 0 & 1 & 10 & 19 & 13 & 24 & 10 & 4 & 3 & 1 & 0 & 0 & 0 & 85 \\
\hline & $\begin{array}{l}\% \text { of } \\
\text { Total }\end{array}$ & $\begin{array}{r}0.0 \\
\%\end{array}$ & $.1 \%$ & $1.2 \%$ & $2.3 \%$ & $1.6 \%$ & $2.9 \%$ & $1.2 \%$ & $.5 \%$ & $.4 \%$ & $.1 \%$ & $\begin{array}{r}0.0 \\
\%\end{array}$ & $\begin{array}{r}0.0 \\
\%\end{array}$ & $\begin{array}{r}0.0 \\
\%\end{array}$ & $10.2 \%$ \\
\hline \multirow{3}{*}{$\begin{array}{l}20 K- \\
24999\end{array}$} & Count & 0 & 1 & 8 & 22 & 30 & 15 & 9 & 3 & 0 & 1 & 0 & 0 & 0 & 89 \\
\hline & $\begin{array}{l}\% \text { of } \\
\text { Total }\end{array}$ & $\begin{array}{r}0.0 \\
\%\end{array}$ & $.1 \%$ & $1.0 \%$ & $2.6 \%$ & $3.6 \%$ & $1.8 \%$ & $1.1 \%$ & $.4 \%$ & $\begin{array}{r}0.0 \\
\%\end{array}$ & $.1 \%$ & $\begin{array}{r}0.0 \\
\%\end{array}$ & $\begin{array}{r}0.0 \\
\%\end{array}$ & $\begin{array}{r}0.0 \\
\%\end{array}$ & $10.7 \%$ \\
\hline & Count & 0 & 0 & 3 & 15 & 12 & 16 & 7 & 5 & 2 & 0 & 0 & 0 & 0 & 60 \\
\hline
\end{tabular}




\begin{tabular}{|c|c|c|c|c|c|c|c|c|c|c|c|c|c|c|c|}
\hline \multirow{2}{*}{\multicolumn{2}{|c|}{ Income level }} & \multicolumn{13}{|c|}{ Number in household: Wave 1} & \multirow[b]{2}{*}{ Total } \\
\hline & & 0 & 1 & 2 & 3 & 4 & 5 & 6 & 7 & 8 & 9 & 10 & 13 & 17 & \\
\hline \multirow{5}{*}{$\begin{array}{l}25 \mathrm{~K}- \\
29999 \\
30 \mathrm{~K}- \\
34999\end{array}$} & $\%$ of & 0.0 & 0.0 & \multirow{2}{*}{$.4 \%$} & \multirow{2}{*}{$1.8 \%$} & \multirow{2}{*}{$1.4 \%$} & \multirow{2}{*}{$1.9 \%$} & \multirow{2}{*}{$.8 \%$} & \multirow[b]{2}{*}{$.6 \%$} & \multirow{2}{*}{$.2 \%$} & 0.0 & 0.0 & 0.0 & 0.0 & \multirow{2}{*}{$7.2 \%$} \\
\hline & Total & $\%$ & $\%$ & & & & & & & & $\%$ & $\%$ & $\%$ & $\%$ & \\
\hline & Count & 0 & 0 & 3 & 7 & 11 & 9 & 5 & 4 & 1 & 1 & 0 & 0 & 0 & 41 \\
\hline & $\%$ of & 0.0 & 0.0 & \multirow{2}{*}{$.4 \%$} & \multirow{2}{*}{$.8 \%$} & \multirow{2}{*}{$1.3 \%$} & \multirow{2}{*}{$1.1 \%$} & \multirow{2}{*}{$.6 \%$} & \multirow{2}{*}{$.5 \%$} & \multirow{2}{*}{$.1 \%$} & \multirow{2}{*}{$.1 \%$} & 0.0 & 0.0 & 0.0 & \multirow{2}{*}{$4.9 \%$} \\
\hline & Total & $\%$ & $\%$ & & & & & & & & & $\%$ & $\%$ & $\%$ & \\
\hline \multirow{3}{*}{$\begin{array}{l}35 \mathrm{~K}- \\
39999\end{array}$} & Count & 0 & 0 & 2 & 10 & 13 & 8 & 5 & 4 & 0 & 2 & 0 & 0 & 0 & 44 \\
\hline & $\%$ of & 0.0 & 0.0 & \multirow{2}{*}{$.2 \%$} & \multirow{2}{*}{$1.2 \%$} & \multirow{2}{*}{$1.6 \%$} & \multirow{2}{*}{$1.0 \%$} & \multirow{2}{*}{$.6 \%$} & \multirow{2}{*}{$.5 \%$} & 0.0 & \multirow{2}{*}{$.2 \%$} & 0.0 & 0.0 & 0.0 & \multirow{2}{*}{$5.3 \%$} \\
\hline & Total & $\%$ & $\%$ & & & & & & & $\%$ & & $\%$ & $\%$ & $\%$ & \\
\hline \multirow{2}{*}{$\begin{array}{l}40 K- \\
44999\end{array}$} & Count & 0 & 0 & 1 & 3 & 6 & 7 & 4 & 1 & 1 & 1 & 0 & 0 & 0 & 24 \\
\hline & $\%$ of & 0.0 & 0.0 & $.1 \%$ & $.4 \%$ & $.7 \%$ & $.8 \%$ & $.5 \%$ & $.1 \%$ & $.1 \%$ & $.1 \%$ & 0.0 & 0.0 & 0.0 & $2.9 \%$ \\
\hline $45 \mathrm{~K}-$ & Count & $\begin{array}{r}10 \\
0\end{array}$ & 0 & 0 & 5 & 2 & 6 & 6 & 3 & 0 & 0 & 0 & 0 & 0 & 22 \\
\hline 49999 & $\%$ of & 0.0 & 0.0 & $00 \%$ & $6 \%$ & $2 \%$ & $7 \%$ & $7 \%$ & $4 \%$ & 0.0 & 0.0 & 0.0 & 0.0 & 0.0 & $26 \%$ \\
\hline & Total & $\%$ & $\%$ & $0.0 \%$ & $.0 \%$ & $.2 \%$ & $.1 \%$ & $.1 \%$ & $.4 \%$ & $\%$ & $\%$ & $\%$ & $\%$ & $\%$ & $2.0 \%$ \\
\hline$>50 \mathrm{~K}$ & Count & 0 & 1 & 3 & 13 & 21 & 17 & 7 & 10 & 5 & 2 & 2 & 1 & 1 & 83 \\
\hline & $\begin{array}{l}\% \text { of } \\
\text { Total }\end{array}$ & $\begin{array}{r}0.0 \\
\%\end{array}$ & $.1 \%$ & $.4 \%$ & $1.6 \%$ & $2.5 \%$ & $2.0 \%$ & $.8 \%$ & $\begin{array}{r}1.2 \\
\%\end{array}$ & $.6 \%$ & $.2 \%$ & $.2 \%$ & $.1 \%$ & $.1 \%$ & $10.0 \%$ \\
\hline & $\begin{array}{l}\text { TOTA } \\
\mathrm{L}\end{array}$ & 1 & 8 & 93 & 177 & 197 & 151 & 83 & 41 & 18 & 9 & 2 & 2 & 1 & 834 \\
\hline & $\%$ of & $1 \%$ & 1.0 & 11.2 & 21.2 & 23.6 & 18.1 & 10.0 & 4.9 & 2.2 & 1.1 & $.2 \%$ & $.2 \%$ & $1 \%$ & 100.0 \\
\hline & Total & $.1 \%$ & $\%$ & $\%$ & $\%$ & $\%$ & $\%$ & $\%$ & $\%$ & $\%$ & $\%$ & $.2 \%$ & $.2 \%$ & $.1 \%$ & \\
\hline
\end{tabular}

Note. Shaded region indicates meeting poverty level guidelines using HHS standards

Table D2

\begin{tabular}{|c|c|c|c|c|c|c|c|c|c|c|c|c|c|}
\hline \multirow{2}{*}{ Income level } & \multicolumn{12}{|c|}{ Number in household: Wave 3} & \multirow[b]{2}{*}{ Total } \\
\hline & 2 & 3 & 4 & 5 & 6 & 7 & 8 & 9 & 10 & 11 & 12 & 56 & \\
\hline & 0 & 0 & 0 & 0 & 0 & 0 & 0 & 0 & 0 & 0 & 0 & 0 & 135 \\
\hline
\end{tabular}




\begin{tabular}{|c|c|c|c|c|c|c|c|c|c|c|c|c|c|c|c|}
\hline \multirow{2}{*}{ Income level } & & \multicolumn{13}{|c|}{ Number in household: Wave 3} & \multirow[b]{2}{*}{ Total } \\
\hline & & 1 & 2 & 3 & 4 & 5 & 6 & 7 & 8 & 9 & 10 & 11 & 12 & 56 & \\
\hline $\begin{array}{l}\text { NON } \\
\text { INTERVIE } \\
\text { W }\end{array}$ & $\begin{array}{l}\% \text { of } \\
\text { Total }\end{array}$ & $\begin{array}{r}0.0 \\
\%\end{array}$ & $\begin{array}{r}0.0 \\
\%\end{array}$ & $0.0 \%$ & $0.0 \%$ & $0.0 \%$ & $0.0 \%$ & $\begin{array}{r}0.0 \\
\%\end{array}$ & $\begin{array}{r}0.0 \\
\%\end{array}$ & $\begin{array}{r}0.0 \\
\%\end{array}$ & $\begin{array}{r}0.0 \\
\%\end{array}$ & $\begin{array}{r}0.0 \\
\%\end{array}$ & $\begin{array}{r}0.0 \\
\%\end{array}$ & $\begin{array}{r}0.0 \\
\%\end{array}$ & $16.2 \%$ \\
\hline \multirow[t]{2}{*}{ REFUSED } & Count & 0 & 0 & 0 & 0 & 0 & 0 & 0 & 0 & 0 & 0 & 0 & 0 & 0 & 16 \\
\hline & $\begin{array}{l}\% \text { of } \\
\text { Total }\end{array}$ & $\begin{array}{r}0.0 \\
\%\end{array}$ & $\begin{array}{r}0.0 \\
\%\end{array}$ & $0.0 \%$ & $0.0 \%$ & $0.0 \%$ & $0.0 \%$ & $\begin{array}{r}0.0 \\
\%\end{array}$ & $\begin{array}{r}0.0 \\
\%\end{array}$ & $\begin{array}{r}0.0 \\
\%\end{array}$ & $\begin{array}{r}0.0 \\
\%\end{array}$ & $\begin{array}{r}0.0 \\
\%\end{array}$ & $\begin{array}{r}0.0 \\
\%\end{array}$ & $\begin{array}{r}0.0 \\
\%\end{array}$ & $1.9 \%$ \\
\hline \multirow{2}{*}{$\begin{array}{l}\text { DONT } \\
\text { KNOW }\end{array}$} & Count & 0 & 1 & 3 & 0 & 3 & 3 & 1 & 0 & 0 & 0 & 0 & 0 & 0 & 13 \\
\hline & $\begin{array}{l}\% \text { of } \\
\text { Total }\end{array}$ & $\begin{array}{r}0.0 \\
\%\end{array}$ & $.1 \%$ & $.4 \%$ & $0.0 \%$ & $.4 \%$ & $.4 \%$ & $.1 \%$ & $\begin{array}{r}0.0 \\
\%\end{array}$ & $\begin{array}{r}0.0 \\
\%\end{array}$ & $\begin{array}{r}0.0 \\
\%\end{array}$ & $\begin{array}{r}0.0 \\
\%\end{array}$ & $\begin{array}{r}0.0 \\
\%\end{array}$ & $\begin{array}{r}0.0 \\
\%\end{array}$ & $1.6 \%$ \\
\hline \multirow[t]{2}{*}{$<5000$} & Count & 1 & 9 & 9 & 9 & 6 & 0 & 1 & 0 & 0 & 0 & 0 & 0 & 1 & 36 \\
\hline & $\begin{array}{l}\% \text { of } \\
\text { Total }\end{array}$ & $.1 \%$ & $\begin{array}{r}1.1 \\
\%\end{array}$ & $1.1 \%$ & $1.1 \%$ & $.7 \%$ & $0.0 \%$ & $.1 \%$ & $\begin{array}{r}0.0 \\
\%\end{array}$ & $\begin{array}{r}0.0 \\
\%\end{array}$ & $\begin{array}{r}0.0 \\
\%\end{array}$ & $\begin{array}{r}0.0 \\
\%\end{array}$ & $\begin{array}{r}0.0 \\
\%\end{array}$ & $.1 \%$ & $4.3 \%$ \\
\hline \multirow[t]{2}{*}{ 5K-9999 } & Count & 1 & 16 & 18 & 28 & 18 & 4 & 1 & 3 & 1 & 0 & 0 & 0 & 0 & 90 \\
\hline & $\begin{array}{l}\% \text { of } \\
\text { Total }\end{array}$ & $.1 \%$ & $\begin{array}{r}1.9 \\
\%\end{array}$ & $2.2 \%$ & $3.4 \%$ & $2.2 \%$ & $.5 \%$ & $.1 \%$ & $.4 \%$ & $.1 \%$ & $\begin{array}{r}0.0 \\
\%\end{array}$ & $\begin{array}{r}0.0 \\
\%\end{array}$ & $\begin{array}{r}0.0 \\
\%\end{array}$ & $\begin{array}{r}0.0 \\
\%\end{array}$ & $10.8 \%$ \\
\hline \multirow[t]{2}{*}{$10 \mathrm{~K}-14999$} & Count & 0 & 15 & 24 & 31 & 26 & 16 & 2 & 2 & 0 & 1 & 0 & 0 & 0 & 117 \\
\hline & $\begin{array}{l}\% \text { of } \\
\text { Total }\end{array}$ & $\begin{array}{r}0.0 \\
\%\end{array}$ & $\begin{array}{r}1.8 \\
\%\end{array}$ & $2.9 \%$ & $3.7 \%$ & $3.1 \%$ & $1.9 \%$ & $.2 \%$ & $.2 \%$ & $\begin{array}{r}0.0 \\
\%\end{array}$ & $.1 \%$ & $\begin{array}{r}0.0 \\
\%\end{array}$ & $\begin{array}{r}0.0 \\
\%\end{array}$ & $\begin{array}{r}0.0 \\
\%\end{array}$ & $14.0 \%$ \\
\hline \multirow[t]{2}{*}{$15 K-19999$} & Count & 0 & 12 & 18 & 28 & 18 & 12 & 8 & 2 & 0 & 1 & 1 & 0 & 0 & 100 \\
\hline & $\begin{array}{l}\% \text { of } \\
\text { Total }\end{array}$ & $\begin{array}{r}0.0 \\
\%\end{array}$ & $\begin{array}{r}1.4 \\
\%\end{array}$ & $2.2 \%$ & $3.4 \%$ & $2.2 \%$ & $1.4 \%$ & $\begin{array}{r}1.0 \\
\%\end{array}$ & $.2 \%$ & $\begin{array}{r}0.0 \\
\%\end{array}$ & $.1 \%$ & $.1 \%$ & $\begin{array}{r}0.0 \\
\%\end{array}$ & $\begin{array}{r}0.0 \\
\%\end{array}$ & $12.0 \%$ \\
\hline \multirow[t]{2}{*}{$20 \mathrm{~K}-24999$} & Count & 0 & 6 & 13 & 16 & 16 & 7 & 3 & 0 & 1 & 0 & 0 & 1 & 0 & 63 \\
\hline & $\begin{array}{l}\% \text { of } \\
\text { Total }\end{array}$ & $\begin{array}{r}0.0 \\
\%\end{array}$ & $.7 \%$ & $1.6 \%$ & $1.9 \%$ & $1.9 \%$ & $.8 \%$ & $.4 \%$ & $\begin{array}{r}0.0 \\
\%\end{array}$ & $.1 \%$ & $\begin{array}{r}0.0 \\
\%\end{array}$ & $\begin{array}{r}0.0 \\
\%\end{array}$ & $.1 \%$ & $\begin{array}{r}0.0 \\
\%\end{array}$ & $7.6 \%$ \\
\hline \multirow[t]{2}{*}{$25 \mathrm{~K}-29999$} & Count & 0 & 4 & 18 & 9 & 13 & 5 & 4 & 3 & 1 & 0 & 1 & 0 & 0 & 58 \\
\hline & $\begin{array}{l}\% \text { of } \\
\text { Total }\end{array}$ & $\begin{array}{r}0.0 \\
\%\end{array}$ & $.5 \%$ & $2.2 \%$ & $1.1 \%$ & $1.6 \%$ & $.6 \%$ & $.5 \%$ & $.4 \%$ & $.1 \%$ & $\begin{array}{r}0.0 \\
\%\end{array}$ & $.1 \%$ & $\begin{array}{r}0.0 \\
\%\end{array}$ & $\begin{array}{r}0.0 \\
\%\end{array}$ & $7.0 \%$ \\
\hline \multirow[t]{2}{*}{$30 \mathrm{~K}-34999$} & Count & 0 & 2 & 6 & 14 & 8 & 6 & 1 & 0 & 0 & 1 & 0 & 0 & 0 & 38 \\
\hline & $\begin{array}{l}\% \text { of } \\
\text { Total }\end{array}$ & $\begin{array}{r}0.0 \\
\%\end{array}$ & $.2 \%$ & $.7 \%$ & $1.7 \%$ & $1.0 \%$ & $.7 \%$ & $.1 \%$ & $\begin{array}{r}0.0 \\
\%\end{array}$ & $\begin{array}{r}0.0 \\
\%\end{array}$ & $.1 \%$ & $\begin{array}{r}0.0 \\
\%\end{array}$ & $\begin{array}{r}0.0 \\
\%\end{array}$ & $\begin{array}{r}0.0 \\
\%\end{array}$ & $4.6 \%$ \\
\hline
\end{tabular}




\begin{tabular}{|c|c|c|c|c|c|c|c|c|c|c|c|c|c|c|c|}
\hline \multirow{2}{*}{ Income level } & & \multicolumn{13}{|c|}{ Number in household: Wave 3} & \multirow[b]{2}{*}{ Total } \\
\hline & & 1 & 2 & 3 & 4 & 5 & 6 & 7 & 8 & 9 & 10 & 11 & 12 & 56 & \\
\hline \multirow[t]{2}{*}{$35 \mathrm{~K}-39999$} & Count & 0 & 1 & 4 & 5 & 12 & 6 & 2 & 1 & 0 & 0 & 0 & 0 & 0 & 31 \\
\hline & $\begin{array}{l}\% \text { of } \\
\text { Total }\end{array}$ & $\begin{array}{r}0.0 \\
\%\end{array}$ & $.1 \%$ & $.5 \%$ & $.6 \%$ & $1.4 \%$ & $.7 \%$ & $.2 \%$ & $.1 \%$ & $\begin{array}{r}0.0 \\
\%\end{array}$ & $\begin{array}{r}0.0 \\
\%\end{array}$ & $\begin{array}{r}0.0 \\
\%\end{array}$ & $\begin{array}{r}0.0 \\
\%\end{array}$ & $\begin{array}{r}0.0 \\
\%\end{array}$ & $3.7 \%$ \\
\hline \multirow[t]{2}{*}{ 40K-44999 } & Count & 0 & 1 & 7 & 9 & 5 & 7 & 4 & 1 & 0 & 0 & 0 & 0 & 0 & 34 \\
\hline & $\begin{array}{l}\% \text { of } \\
\text { Total }\end{array}$ & $\begin{array}{r}0.0 \\
\%\end{array}$ & $.1 \%$ & $.8 \%$ & $1.1 \%$ & $.6 \%$ & $.8 \%$ & $.5 \%$ & $.1 \%$ & $\begin{array}{r}0.0 \\
\%\end{array}$ & $\begin{array}{r}0.0 \\
\%\end{array}$ & $\begin{array}{r}0.0 \\
\%\end{array}$ & $\begin{array}{r}0.0 \\
\%\end{array}$ & $\begin{array}{r}0.0 \\
\%\end{array}$ & $4.1 \%$ \\
\hline \multirow[t]{2}{*}{$45 \mathrm{~K}-49999$} & Count & 0 & 2 & 5 & 3 & 3 & 3 & 1 & 1 & 1 & 1 & 0 & 0 & 0 & 20 \\
\hline & $\begin{array}{l}\% \text { of } \\
\text { Total }\end{array}$ & $\begin{array}{r}0.0 \\
\%\end{array}$ & $.2 \%$ & $.6 \%$ & $.4 \%$ & $.4 \%$ & $.4 \%$ & $.1 \%$ & $.1 \%$ & $.1 \%$ & $.1 \%$ & $\begin{array}{r}0.0 \\
\%\end{array}$ & $\begin{array}{r}0.0 \\
\%\end{array}$ & $\begin{array}{r}0.0 \\
\%\end{array}$ & $2.4 \%$ \\
\hline \multirow[t]{4}{*}{$>50 \mathrm{~K}$} & Count & 0 & 3 & 13 & 23 & 18 & 14 & 4 & 4 & 1 & 3 & 0 & 0 & 0 & 83 \\
\hline & $\begin{array}{l}\% \text { of } \\
\text { Total }\end{array}$ & $\begin{array}{r}0.0 \\
\%\end{array}$ & $.4 \%$ & $1.6 \%$ & $2.8 \%$ & $2.2 \%$ & $1.7 \%$ & $.5 \%$ & $.5 \%$ & $.1 \%$ & $.4 \%$ & $\begin{array}{r}0.0 \\
\%\end{array}$ & $\begin{array}{r}0.0 \\
\%\end{array}$ & $\begin{array}{r}0.0 \\
\%\end{array}$ & $10.0 \%$ \\
\hline & $\begin{array}{l}\text { TOTA } \\
\mathrm{L}\end{array}$ & 2 & 72 & 138 & 175 & 146 & 83 & 32 & 17 & 5 & 7 & 2 & 1 & 1 & 834 \\
\hline & $\begin{array}{l}\% \text { of } \\
\text { Total }\end{array}$ & $.2 \%$ & $\begin{array}{r}8.6 \\
\%\end{array}$ & $\begin{array}{r}16.5 \\
\%\end{array}$ & $\begin{array}{r}21.0 \\
\%\end{array}$ & $\begin{array}{r}17.5 \\
\%\end{array}$ & $\begin{array}{r}10.0 \\
\%\end{array}$ & $\begin{array}{r}3.8 \\
\%\end{array}$ & $\begin{array}{r}2.0 \\
\%\end{array}$ & $.6 \%$ & $.8 \%$ & $.2 \%$ & $.1 \%$ & $.1 \%$ & $\begin{array}{r}100.0 \\
\%\end{array}$ \\
\hline
\end{tabular}

Note. Shaded region indicates meeting poverty level guidelines using HHS standards

Table D3

\begin{tabular}{|c|c|c|c|c|c|c|c|c|c|c|c|c|c|}
\hline \multirow{2}{*}{ Income level } & & \multicolumn{11}{|c|}{ Number in household: Wave 4} & \multirow[b]{2}{*}{ Total } \\
\hline & & 1 & 2 & 3 & 4 & 5 & 6 & 7 & 8 & 9 & 10 & 11 & \\
\hline \multirow[t]{2}{*}{ NON INTERVIEW } & Count & 0 & 0 & 0 & 0 & 0 & 0 & 0 & 0 & 0 & 0 & 0 & 133 \\
\hline & $\%$ of Total & $0.0 \%$ & $0.0 \%$ & $0.0 \%$ & $0.0 \%$ & $0.0 \%$ & $0.0 \%$ & $0.0 \%$ & $0.0 \%$ & $0.0 \%$ & $0.0 \%$ & $0.0 \%$ & $15.9 \%$ \\
\hline \multirow[t]{2}{*}{ REFUSED } & Count & 0 & 0 & 0 & 0 & 0 & 0 & 0 & 0 & 0 & 0 & 0 & 11 \\
\hline & $\%$ of Total & $0.0 \%$ & $0.0 \%$ & $0.0 \%$ & $0.0 \%$ & $0.0 \%$ & $0.0 \%$ & $0.0 \%$ & $0.0 \%$ & $0.0 \%$ & $0.0 \%$ & $0.0 \%$ & $1.3 \%$ \\
\hline \multirow[t]{2}{*}{ DONT KNOW } & Count & 0 & 1 & 3 & 2 & 3 & 0 & 0 & 0 & 0 & 0 & 0 & 11 \\
\hline & $\%$ of Total & $0.0 \%$ & $.1 \%$ & $.4 \%$ & $.2 \%$ & $.4 \%$ & $0.0 \%$ & $0.0 \%$ & $0.0 \%$ & $0.0 \%$ & $0.0 \%$ & $0.0 \%$ & $1.3 \%$ \\
\hline$<5000$ & Count & 0 & 8 & 7 & 10 & 4 & 2 & 1 & 0 & 0 & 0 & 0 & 32 \\
\hline
\end{tabular}




\begin{tabular}{|c|c|c|c|c|c|c|c|c|c|c|c|c|c|}
\hline \multirow{3}{*}{ Income level } & & \multicolumn{11}{|c|}{ Number in household: Wave 4} & \multirow[b]{2}{*}{ Total } \\
\hline & & 1 & 2 & 3 & 4 & 5 & 6 & 7 & 8 & 9 & 10 & 11 & \\
\hline & $\%$ of Total & $0.0 \%$ & $1.0 \%$ & $.8 \%$ & $1.2 \%$ & $.5 \%$ & $.2 \%$ & $.1 \%$ & $0.0 \%$ & $0.0 \%$ & $0.0 \%$ & $0.0 \%$ & $3.8 \%$ \\
\hline \multirow[t]{2}{*}{ 5K-9999 } & Count & 2 & 14 & 30 & 18 & 18 & 5 & 1 & 1 & 0 & 1 & 0 & 90 \\
\hline & $\%$ of Total & $.2 \%$ & $1.7 \%$ & $3.6 \%$ & $2.2 \%$ & $2.2 \%$ & $.6 \%$ & $.1 \%$ & $.1 \%$ & $0.0 \%$ & $.1 \%$ & $0.0 \%$ & $10.8 \%$ \\
\hline \multirow[t]{2}{*}{ 10K-14999 } & Count & 1 & 13 & 17 & 35 & 21 & 10 & 4 & 0 & 1 & 0 & 0 & 102 \\
\hline & $\%$ of Total & $.1 \%$ & $1.6 \%$ & $2.0 \%$ & $4.2 \%$ & $2.5 \%$ & $1.2 \%$ & $.5 \%$ & $0.0 \%$ & $.1 \%$ & $0.0 \%$ & $0.0 \%$ & $12.2 \%$ \\
\hline \multirow[t]{2}{*}{ 15K-19999 } & Count & 0 & 10 & 17 & 27 & 20 & 11 & 6 & 1 & 1 & 0 & 0 & 93 \\
\hline & $\%$ of Total & $0.0 \%$ & $1.2 \%$ & $2.0 \%$ & $3.2 \%$ & $2.4 \%$ & $1.3 \%$ & $.7 \%$ & $.1 \%$ & $.1 \%$ & $0.0 \%$ & $0.0 \%$ & $11.2 \%$ \\
\hline \multirow[t]{2}{*}{ 20K-24999 } & Count & 0 & 7 & 18 & 12 & 17 & 11 & 4 & 1 & 2 & 0 & 0 & 72 \\
\hline & $\%$ of Total & $0.0 \%$ & $.8 \%$ & $2.2 \%$ & $1.4 \%$ & $2.0 \%$ & $1.3 \%$ & $.5 \%$ & $.1 \%$ & $.2 \%$ & $0.0 \%$ & $0.0 \%$ & $8.6 \%$ \\
\hline \multirow[t]{2}{*}{ 25K-29999 } & Count & 0 & 1 & 13 & 12 & 11 & 5 & 2 & 2 & 0 & 0 & 0 & 46 \\
\hline & $\%$ of Total & $0.0 \%$ & $.1 \%$ & $1.6 \%$ & $1.4 \%$ & $1.3 \%$ & $.6 \%$ & $.2 \%$ & $.2 \%$ & $0.0 \%$ & $0.0 \%$ & $0.0 \%$ & $5.5 \%$ \\
\hline \multirow[t]{2}{*}{ 30K-34999 } & Count & 0 & 3 & 8 & 8 & 11 & 6 & 4 & 1 & 1 & 0 & 0 & 42 \\
\hline & $\%$ of Total & $0.0 \%$ & $.4 \%$ & $1.0 \%$ & $1.0 \%$ & $1.3 \%$ & $.7 \%$ & $.5 \%$ & $.1 \%$ & $.1 \%$ & $0.0 \%$ & $0.0 \%$ & $5.0 \%$ \\
\hline \multirow[t]{2}{*}{ 35K-39999 } & Count & 0 & 1 & 4 & 14 & 18 & 5 & 1 & 3 & 0 & 0 & 0 & 46 \\
\hline & $\%$ of Total & $0.0 \%$ & $.1 \%$ & $.5 \%$ & $1.7 \%$ & $2.2 \%$ & $.6 \%$ & $.1 \%$ & $.4 \%$ & $0.0 \%$ & $0.0 \%$ & $0.0 \%$ & $5.5 \%$ \\
\hline \multirow[t]{2}{*}{ 40K-44999 } & Count & 0 & 3 & 6 & 9 & 5 & 4 & 1 & 1 & 0 & 0 & 1 & 30 \\
\hline & $\%$ of Total & $0.0 \%$ & $.4 \%$ & $.7 \%$ & $1.1 \%$ & $.6 \%$ & $.5 \%$ & $.1 \%$ & $.1 \%$ & $0.0 \%$ & $0.0 \%$ & $.1 \%$ & $3.6 \%$ \\
\hline \multirow[t]{2}{*}{ 45K-49999 } & Count & 0 & 1 & 2 & 10 & 4 & 2 & 2 & 0 & 0 & 0 & 0 & 21 \\
\hline & $\%$ of Total & $0.0 \%$ & $.1 \%$ & $.2 \%$ & $1.2 \%$ & $.5 \%$ & $.2 \%$ & $.2 \%$ & $0.0 \%$ & $0.0 \%$ & $0.0 \%$ & $0.0 \%$ & $2.5 \%$ \\
\hline \multirow[t]{4}{*}{$>50 \mathrm{~K}$} & Count & 0 & 1 & 16 & 31 & 25 & 14 & 10 & 4 & 3 & 1 & 0 & 105 \\
\hline & $\%$ of Total & $0.0 \%$ & $.1 \%$ & $1.9 \%$ & $3.7 \%$ & $3.0 \%$ & $1.7 \%$ & $1.2 \%$ & $.5 \%$ & $.4 \%$ & $.1 \%$ & $0.0 \%$ & $12.6 \%$ \\
\hline & Count & 3 & 63 & 141 & 188 & 157 & 75 & 36 & 14 & 8 & 2 & 1 & 834 \\
\hline & $\%$ of Total & $.4 \%$ & $7.6 \%$ & $16.9 \%$ & $22.5 \%$ & $18.8 \%$ & $9.0 \%$ & $4.3 \%$ & $1.7 \%$ & $1.0 \%$ & $.2 \%$ & $.1 \%$ & $100.0 \%$ \\
\hline
\end{tabular}

Note. Shaded region indicates meeting poverty level guidelines using HHS standards 


\section{Appendix E: Missing Data Pattern Frequencies}

Table E1

\begin{tabular}{|c|c|c|c|c|c|}
\hline Pattern & Frequency & Pattern & Frequency & Pattern & Frequency \\
\hline 1 & 399 & 39 & 3 & 77 & 1 \\
\hline 2 & 1 & 40 & 1 & 78 & 5 \\
\hline 3 & 2 & 41 & 1 & 79 & 5 \\
\hline 4 & 1 & 42 & 4 & 80 & 1 \\
\hline 5 & 2 & 43 & 1 & 81 & 1 \\
\hline 6 & 1 & 44 & 1 & 82 & 1 \\
\hline 7 & 1 & 45 & 1 & 83 & 8 \\
\hline 8 & 1 & 46 & 1 & 84 & 1 \\
\hline 9 & 1 & 47 & 1 & 85 & 25 \\
\hline 10 & 1 & 48 & 83 & 86 & 1 \\
\hline 11 & 1 & 49 & 1 & 87 & 1 \\
\hline 12 & 1 & 50 & 2 & 88 & 1 \\
\hline 13 & 1 & 51 & 1 & 89 & 1 \\
\hline 14 & 1 & 52 & 25 & 90 & 1 \\
\hline 15 & 1 & 53 & 1 & 91 & 2 \\
\hline 16 & 14 & 54 & 1 & 92 & 1 \\
\hline 17 & 1 & 55 & 1 & 93 & 1 \\
\hline 18 & 1 & 56 & 1 & 94 & 1 \\
\hline 19 & 1 & 57 & 19 & 95 & 1 \\
\hline 20 & 5 & 58 & 2 & 96 & 1 \\
\hline 21 & 1 & 59 & 1 & 97 & 1 \\
\hline 22 & 2 & 60 & 1 & 98 & 15 \\
\hline 23 & 2 & 61 & 4 & 99 & 1 \\
\hline 24 & 1 & 62 & 1 & 100 & 2 \\
\hline 25 & 23 & 63 & 1 & 101 & 1 \\
\hline 26 & 1 & 64 & 1 & 102 & 1 \\
\hline 27 & 1 & 65 & 1 & 103 & 1 \\
\hline 28 & 1 & 66 & 1 & 104 & 1 \\
\hline 29 & 1 & 67 & 1 & 105 & 4 \\
\hline 30 & 2 & 68 & 19 & 106 & 6 \\
\hline 31 & 1 & 69 & 1 & 107 & 7 \\
\hline 32 & 1 & 70 & 32 & 108 & 1 \\
\hline 33 & 1 & 71 & 1 & 109 & 2 \\
\hline 34 & 1 & 72 & 1 & 110 & 1 \\
\hline 35 & 1 & 73 & 23 & 111 & 1 \\
\hline 36 & 1 & 74 & 1 & 112 & 1 \\
\hline 37 & 1 & 75 & 1 & 113 & 2 \\
\hline 38 & 1 & 76 & 4 & & \\
\hline
\end{tabular}




\section{Appendix F: Overlapping Items on the CBCL and SSRS}

Table F1

\begin{tabular}{ccl}
\hline & \multicolumn{1}{c}{ Anxious/Depressed (CBCL) } \\
\hline Item \# (BC) & Item \# (TC) & \multicolumn{1}{c}{ Item text } \\
14 & 13 & cries a lot \\
29 & 32 & fears certain animals, situations, or places, other than school \\
30 & -- & fears going to school \\
31 & -- & fears he/she might think or do something bad \\
32 & -- & feels he/she has to be perfect \\
33 & -- & feels or complains that no one loves him/her \\
35 & -- & feels worthless or inferior \\
45 & 47 & nervous, highstrung, or tense \\
50 & 87 & too fearful or anxious \\
52 & -- & feels too guilty \\
71 & 68 & self-conscious or easily embarrased \\
91 & -- & talks about killing self \\
112 & -- & worries \\
-- & 10 & clings to adults or too dependent \\
-- & 33 & feelings are easily hurt \\
-- & 37 & gets too upset when separated from parents \\
-- & 43 & looks unhappy without good reason \\
-- & 50 & overtired \\
-- & 73 & shy or timid \\
-- & 90 & unhappy, sad, or depressed \\
-- & 96 & wants a lot of attention \\
\hline
\end{tabular}

Table F2

\begin{tabular}{ccl}
\hline & \multicolumn{2}{c}{ Aggressive (CBCL) } \\
\hline Item \# (BC) & Item \# (TC) & Item text \\
3 & -- & argues a lot \\
16 & -- & cruelty, bullying, or meanness to others \\
19 & -- & demands a lot of attention \\
20 & -- & destroys his/her own things \\
21 & -- & destroys things belonging to family or others \\
22 & -- & disobedient at home \\
23 & -- & disobedient at school \\
37 & 35 & gets in many fights \\
57 & -- & physically attacks people \\
68 & 66 & screams a lot \\
\hline
\end{tabular}




\begin{tabular}{lll}
\hline & \multicolumn{1}{c}{ Aggressive (CBCL) } \\
\hline 86 & -- & stubborn, sullen , or irritable \\
87 & 82 & sudden changes in mood or feelings \\
88 & -- & sulks a lot \\
89 & -- & suspiscious \\
94 & -- & teases a lot \\
95 & 85 & temper tantrums or hot temper \\
97 & -- & threatens people \\
104 & 91 & unusually loud \\
-- & 15 & defiant \\
-- & 16 & demands must be met immediately \\
-- & 20 & disobedient \\
-- & 29 & easily frustrated \\
-- & 30 & easily jealous \\
-- & 40 & hits others \\
-- & 44 & angry moods \\
-- & 58 & punishment does not change behavior \\
-- & 69 & selfish or won't share \\
-- & 97 & whining \\
\hline
\end{tabular}

Table F3

\begin{tabular}{ccl}
\hline & \multicolumn{3}{c}{ Attention (CBCL) } \\
\hline Item \# (BC) & Item \# (TC) & Item text \\
1 & 2 & acts too young for age \\
10 & 6 & can't sit still, restless, or hyperactive \\
13 & -- & confused or seems to be in a fog \\
17 & -- & daydreams or gets lost in thoughts \\
41 & -- & impulsive or acts without thinking \\
61 & -- & poor school work \\
80 & -- & stares blankly \\
8 & 5 & can't concentrate/can't pay attention for long \\
-- & 59 & quickly shifts from one activity to another \\
-- & 77 & stares into space or seems preoccupied \\
-- & 8 & can't stand waiting; wants everything now \\
\hline
\end{tabular}

Table F4

Self-control (SSRS)

$\begin{array}{ccl}\text { Item \# (PS) } & \text { Item \# (PT) } & \text { Item Text } \\ 1 & -- & \text { Follows instructions(NEVR13T) } \\ 11 & 6 & \text { Resp approp when hit by oth ch(NEVR13T) } \\ 14 & 14 & \text { Avoid trouble situations(NEVR13T) }\end{array}$




\begin{tabular}{lcl}
\hline & \multicolumn{1}{c}{ Self-control (SSRS) } \\
\hline 18 & 26 & Control temper in conflict w/R(NEVR13T) \\
19 & 22 & Control tempr when argue oth ch(NEVR13T) \\
21 & -- & Follow rules when play others(NEVR13T) \\
22 & -- & Listen/follow Rs instructions (NEVR13T) \\
28 & -- & Waits turn in games(NEVR13T) \\
35 & 25 & Calmly end disagreements w/R(NEVR13T) \\
39 & 3 & Spk in approp tne of voice @hme(NEVR13T) \\
-- & 17 & Receive criticism well(NEVR13T) \\
-- & 32 & Resp approp teasing(NEVR13T) \\
-- & 36 & Coop w/fam mmbrs w/o being askd(NEVR13T) \\
\hline
\end{tabular}




\section{Appendix G: Final CBCL Items Used in the Study}

Table G1

\begin{tabular}{ccccc}
\hline $\begin{array}{c}\text { Item \# } \\
\text { (BC) }\end{array}$ & $\begin{array}{c}\text { Item \# } \\
(\text { TC })\end{array}$ & Recode & Item text & Subscale \\
\hline 1 & 2 & A1 & $\begin{array}{c}\text { acts too young for age } \\
\text { can't concentrate, can't pay attention for }\end{array}$ & Attention \\
8 & 5 & A2 & Attention \\
10 & 6 & A3 & can't sit still, restless, hyperactive & Attention \\
19 & 96 & A4 & demands a lot of attention & Aggressive \\
20 & 17 & A5 & destroys his/her own things & Aggressive \\
21 & 18 & A6 & destroys things belonging to his/her family & Aggressive \\
22 & 20 & A7 & disobedient at home & Aggressive \\
29 & 32 & A8 & fears certain animals, situations, or places, & Anxious/Depressed \\
37 & 35 & A9 & other than school & Aggressive \\
45 & 47 & A10 & nervous, high-strung, or tense & Anxious/Depressed \\
50 & 87 & A11 & too fearful or anxious & Anxious/Depressed \\
57 & 53 & A12 & physically attacks people & Aggressive \\
68 & 66 & A13 & screams a lot & Aggressive \\
71 & 68 & A14 & self-conscious or easily embarrassed & Anxious/Depressed \\
80 & 77 & A15 & stares blankly & Attention \\
86 & 81 & A16 & stubborn, sullen, or irritable & Aggressive \\
87 & 82 & A17 & sudden changes in mood or feelings & Aggressive \\
88 & 83 & A18 & sulks a lot & Aggressive \\
95 & 85 & A19 & temper tantrums or hot temper & Aggressive \\
104 & 91 & A20 & unusually loud & Aggressive \\
112 & 99 & A21 & worries & Anxious/Depressed \\
\hline
\end{tabular}




\section{Appendix H: Final SSRS Items Used in the Study}

Table H1

\begin{tabular}{cccc}
\hline $\begin{array}{c}\text { Item \# } \\
\text { (PS) }\end{array}$ & $\begin{array}{c}\text { Item \# } \\
\text { (PT) }\end{array}$ & Recode & Item Text \\
\hline 39 & 3 & B1 & inappropriate tone of voice \\
11 & 6 & B2 & inappropriate response when hit \\
14 & 14 & B3 & gets in troubling situations \\
19 & 22 & B4 & temper problems with kids \\
35 & 25 & B5 & doesn't calmly end disagreements \\
18 & 26 & B6 & temper problems with caregiver \\
\hline
\end{tabular}




\section{Appendix I: Boxplot Figures with Outliers}

HOME Cognitive Stimulation
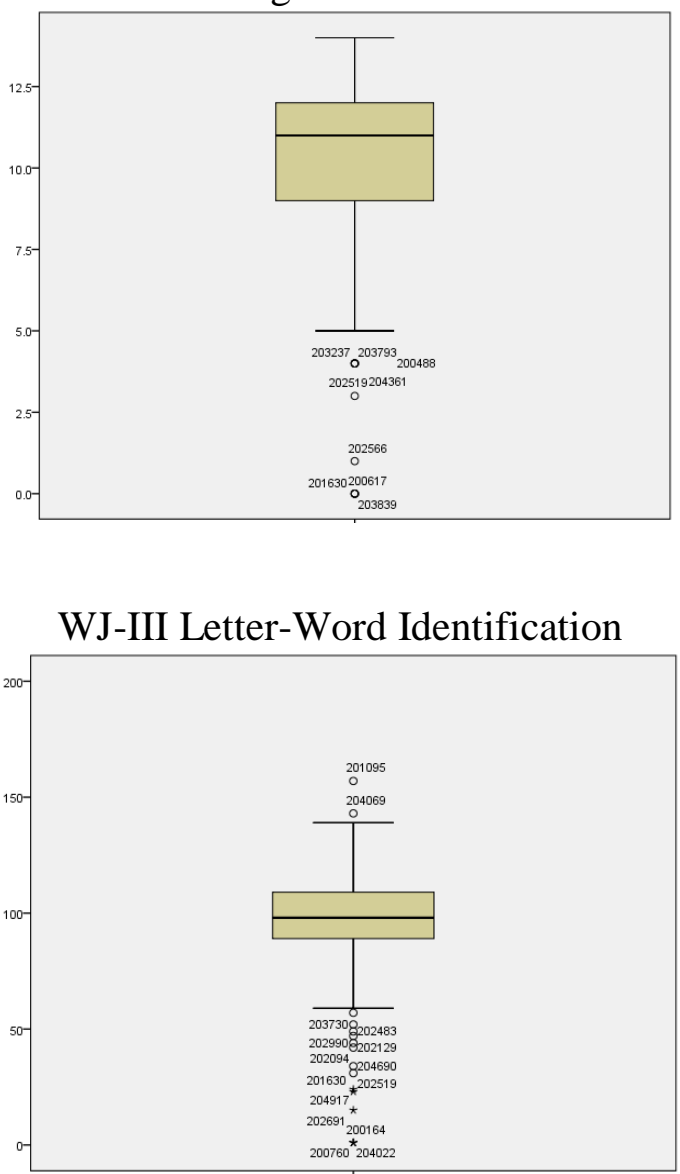

WJ-III Comprehension

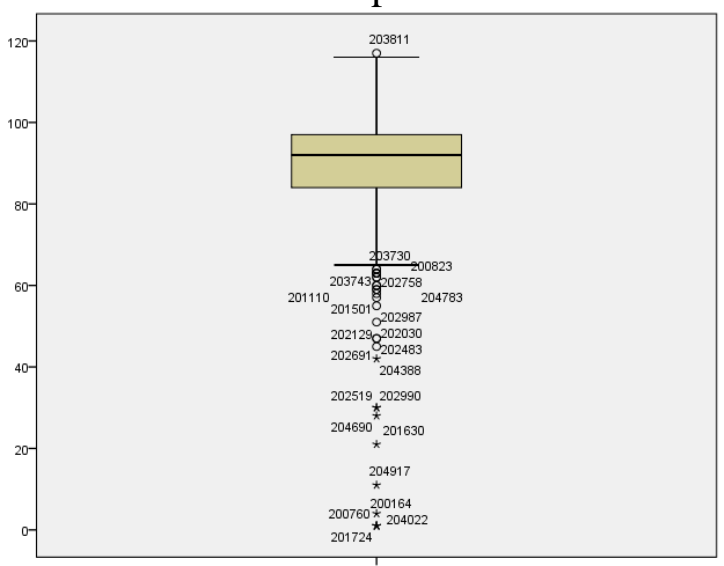

HOME Emotional Support

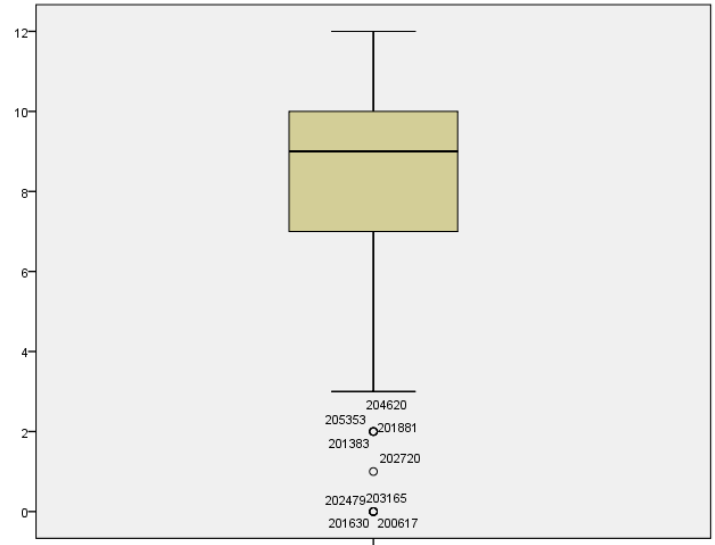

WJ-III Calculation

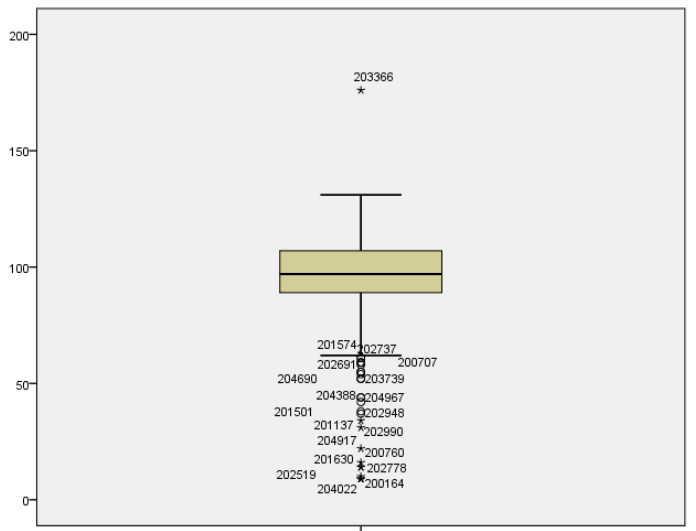

WJ-III Applied Problem

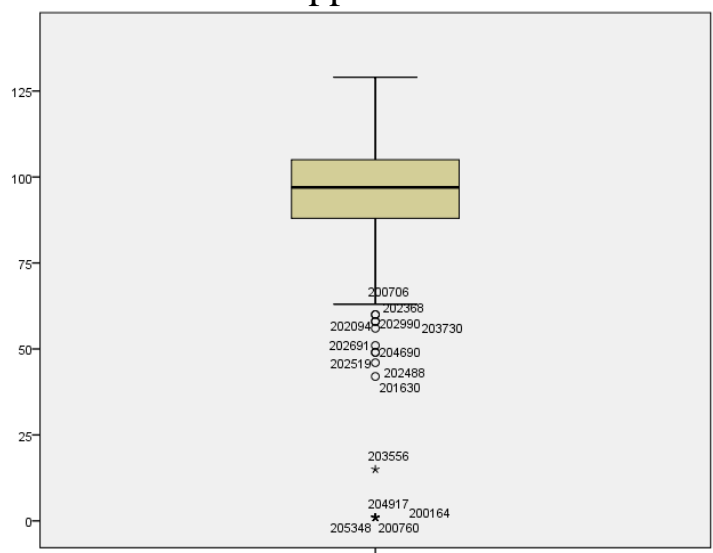




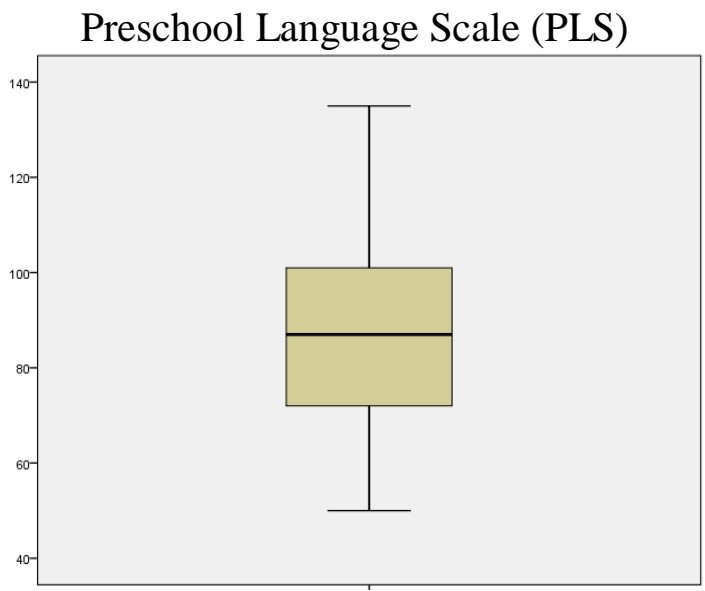

Figure I1. Boxplot figures with outlier cases for the language and context covariates, as well as the distal outcome of academic achievement. 
Appendix J: Intercorrelations of Study Variables

Table J1

\begin{tabular}{|c|c|c|c|c|c|c|c|c|c|c|c|}
\hline & 1. & 2. & 3. & 4. & 5. & 6. & 7. & 8. & 9. & 10. & 11. \\
\hline 1. child gender & 1 & & & & & & & & & & \\
\hline $\begin{array}{l}\text { Type of } \\
\text { maltreatment }\end{array}$ & .008 & 1 & & & & & & & & & \\
\hline $\begin{array}{l}\text { 3. PLS Total Lang } \\
\text { (SS) }\end{array}$ & $.103^{* *}$ & $.117^{* *}$ & 1 & & & & & & & & \\
\hline $\begin{array}{l}\text { W-J Letter- } \\
\text { Word ID (SS) }\end{array}$ & $.096^{*}$ & -.033 & $.350^{* *}$ & 1 & & & & & & & \\
\hline $\begin{array}{l}\text { W-J Calculation } \\
\text { (SS) }\end{array}$ & .063 & .024 & $.304^{* *}$ & $.576^{* *}$ & 1 & & & & & & \\
\hline $\begin{array}{l}\text { W-J Passage } \\
\text { Comp (SS) }\end{array}$ & $.116^{* *}$ & .020 & $.343^{* *}$ & $.788^{* *}$ & $.641^{* *}$ & 1 & & & & & \\
\hline $\begin{array}{l}\text { W-J Applied } \\
\text { Problems (SS) }\end{array}$ & .025 & -.016 & $.315^{* *}$ & $.605^{* *}$ & $.695^{* *}$ & $.692^{* *}$ & 1 & & & & \\
\hline 8. A1w1 & $-.096^{* *}$ & $-.070^{*}$ & $-.164^{* *}$ & $-.180^{* *}$ & $-.144^{* *}$ & $-.222^{* *}$ & $-.172^{* *}$ & 1 & & & \\
\hline 9. $\mathrm{A} 2 \mathrm{w} 1$ & -.049 & -.017 & $-.089^{*}$ & $-.186^{* *}$ & $-.129^{* * *}$ & $-.140^{* *}$ & $-.135^{* *}$ & $.310^{* *}$ & 1 & & \\
\hline 10. $\mathrm{A} 3 \mathrm{w} 1$ & -.030 & .020 & $-.094^{*}$ & $-.162^{* *}$ & $-.123^{* *}$ & $-.124^{* *}$ & $-.140^{* *}$ & $.215^{* *}$ & $.536^{* *}$ & 1 & \\
\hline 11. $\mathrm{A} 4 \mathrm{w} 1$ & .015 & .039 & -.032 & -.067 & -.030 & -.017 & -.044 & $.142^{* *}$ & $.317^{* *}$ & $.358^{* *}$ & 1 \\
\hline 12. A5w1 & $-.107^{* *}$ & .016 & -.071 & -.033 & -.052 & -.014 & -.033 & $.172^{* *}$ & $.327^{* *}$ & $.386^{* *}$ & $.292^{* *}$ \\
\hline 13. A6w1 & -.049 & -.003 & $-.077^{*}$ & -.041 & -.066 & $-.081^{*}$ & $-.083^{*}$ & $.211^{* *}$ & $.325^{* *}$ & $.363^{* *}$ & $.285^{* *}$ \\
\hline 14. $\mathrm{A} 7 \mathrm{w} 1$ & $-.074^{*}$ & .048 & -.034 & -.045 & -.036 & -.021 & -.047 & $.211^{* *}$ & $.404^{* *}$ & $.386^{* *}$ & $.353^{* *}$ \\
\hline 15. A8w1 & .068 & -.016 & -.035 & -.014 & -.005 & .026 & .006 & $.088^{*}$ & $.113^{* *}$ & $.175^{* *}$ & $.103^{* *}$ \\
\hline 16. A9w1 & -.046 & .026 & -.013 & $-.183^{* *}$ & $-.133^{* *}$ & $-.208^{* *}$ & $-.164^{* *}$ & $.121^{* *}$ & $.225^{* *}$ & $.246^{* *}$ & $.232^{* *}$ \\
\hline 17. A10w1 & -.020 & $-.076^{*}$ & -.068 & -.050 & -.007 & -.050 & -.030 & $.296^{* *}$ & $.363^{* *}$ & $.384^{* *}$ & $.281^{* *}$ \\
\hline 18. A11w1 & -.004 & -.030 & -.034 & .004 & .038 & .003 & .006 & $.150^{* *}$ & $.301^{* *}$ & $.308^{* *}$ & $.253^{* *}$ \\
\hline
\end{tabular}




\begin{tabular}{|c|c|c|c|c|c|c|c|c|c|c|c|c|}
\hline & & 1. & 2. & 3. & 4. & 5. & 6. & 7. & 8. & 9. & 10. & 11. \\
\hline & A12w1 & $-.099^{* *}$ & .058 & -.024 & -.027 & $-.083^{*}$ & -.073 & $-.106^{* *}$ & $.151^{* *}$ & $.195^{* *}$ & $.213^{* *}$ & $.212^{* *}$ \\
\hline & A13w1 & -.029 & .057 & $-.085^{*}$ & $-.102^{*}$ & $-.092^{*}$ & $-.088^{*}$ & $-.104^{*}$ & $.162^{* *}$ & $.337^{* *}$ & $.376^{* *}$ & $.336^{* *}$ \\
\hline & A14w1 & .028 & -.008 & .026 & $.091^{*}$ & $.109^{* *}$ & $.092^{*}$ & $.084^{*}$ & .065 & $.117^{* *}$ & $.119^{* *}$ & $.190^{* *}$ \\
\hline & A15w1 & -.015 & -.020 & $-.152^{* *}$ & $-.135^{* *}$ & $-.127^{* *}$ & $-.143^{* *}$ & $-.161^{* *}$ & $.309^{* *}$ & $.252^{* *}$ & $.249^{* *}$ & $.198^{* *}$ \\
\hline & A16w1 & -.022 & .058 & -.055 & -.046 & $-.081^{*}$ & -.026 & -.071 & $.176^{* *}$ & $.370^{* *}$ & $.399^{* *}$ & $.388^{* *}$ \\
\hline & $\mathrm{A} 17 \mathrm{w} 1$ & -.042 & .011 & .001 & -.023 & -.074 & -.015 & -.060 & $.179^{* *}$ & $.342^{* *}$ & $.400^{* *}$ & $.368^{* *}$ \\
\hline & A18w1 & .033 & .027 & .036 & .002 & -.036 & -.013 & -.027 & $.146^{* *}$ & $.303^{* *}$ & $.310^{* *}$ & $.373^{* *}$ \\
\hline & A19w1 & -.042 & .050 & -.027 & -.032 & $-.086^{*}$ & -.016 & -.042 & $.131^{* *}$ & $.330^{* *}$ & $.379^{* *}$ & $.398^{* *}$ \\
\hline 27. & A20w1 & -.024 & .037 & $-.081^{*}$ & $-.097^{*}$ & -.066 & $-.097^{*}$ & $-.085^{*}$ & $.172^{* *}$ & $.298^{* *}$ & $.408^{* *}$ & $.313^{* *}$ \\
\hline & A21w1 & .024 & -.001 & $.078^{*}$ & .033 & .042 & .051 & .065 & $.087^{*}$ & $.105^{* *}$ & $.135^{* *}$ & $.238^{* *}$ \\
\hline 29. & B1w1 & .032 & .009 & $.145^{* *}$ & $.105^{*}$ & .065 & .052 & $.104^{*}$ & $-.153^{* *}$ & $-.208^{* *}$ & $-.199^{* *}$ & $-.161^{* *}$ \\
\hline 30. & B2w1 & $.078^{*}$ & -.038 & .035 & .023 & .026 & .006 & -.010 & $-.102^{* *}$ & $-.150^{* *}$ & $-.165^{* *}$ & $-.144^{* *}$ \\
\hline 31. & B3w1 & $.098^{* *}$ & -.013 & $.143^{* *}$ & $.082^{*}$ & .063 & .061 & .064 & $-.163^{* *}$ & $-.174^{* *}$ & $-.199^{* *}$ & $-.153^{* *}$ \\
\hline 32. & B4w1 & .059 & -.028 & .061 & .068 & .048 & $.084^{*}$ & $.098^{*}$ & $-.123^{* *}$ & $-.158^{* *}$ & $-.198^{* *}$ & $-.197^{* *}$ \\
\hline 33. & B5w1 & $.088^{*}$ & -.060 & $.076^{*}$ & .064 & $.084^{*}$ & .032 & .077 & -.046 & $-.159^{* *}$ & $-.145^{* *}$ & $-.161^{* *}$ \\
\hline 34. & B6w1 & .065 & $-.089^{*}$ & .034 & .018 & .057 & -.002 & .021 & $-.118^{* *}$ & $-.225^{* *}$ & $-.263^{* *}$ & $-.227^{* *}$ \\
\hline & A1w3 & -.064 & -.019 & $-.136^{* *}$ & $-.182^{* *}$ & $-.118^{* *}$ & $-.179^{* *}$ & $-.121^{* *}$ & $.433^{* *}$ & $.190^{* *}$ & $.154^{* *}$ & $.100^{* *}$ \\
\hline 36. & $\mathrm{~A} 2 \mathrm{w} 3$ & $-.095^{*}$ & .018 & $-.162^{* *}$ & $-.156^{* *}$ & $-.103^{*}$ & $-.121^{* *}$ & $-.114^{* *}$ & $.218^{* *}$ & $.393^{* *}$ & $.324^{* *}$ & $.193^{* *}$ \\
\hline 37. & A3w3 & -.049 & .029 & $-.136^{* *}$ & -.070 & -.028 & -.045 & -.012 & $.113^{* *}$ & $.350^{* *}$ & $.398^{* *}$ & $.198^{* *}$ \\
\hline 38. & A4w3 & .040 & .053 & .051 & -.070 & $-.100^{*}$ & -.044 & -.038 & $.113^{* *}$ & $.209^{* *}$ & $.271^{* *}$ & $.373^{* *}$ \\
\hline 39. & A5w3 & $-.172^{* *}$ & .038 & $-.150^{* *}$ & $-.124^{* *}$ & $-.147^{* *}$ & $-.092^{*}$ & -.082 & $.129^{* *}$ & $.263^{* *}$ & $.278^{* *}$ & $.182^{* *}$ \\
\hline 40. & A6w3 & -.056 & .007 & $-.147^{* *}$ & $-.118^{* * *}$ & $-.163^{* *}$ & -.080 & $-.110^{*}$ & $.113^{* *}$ & $.251^{* *}$ & $.278^{* *}$ & $.156^{* *}$ \\
\hline 41. & A7w3 & -.054 & -.026 & -.072 & -.034 & -.081 & .001 & -.003 & $.075^{*}$ & $.268^{* *}$ & $.215^{* *}$ & $.187^{* *}$ \\
\hline 42. & A8w3 & .047 & -.021 & -.015 & -.015 & -.043 & -.042 & -.028 & .050 & .021 & $.099^{* * *}$ & .063 \\
\hline
\end{tabular}




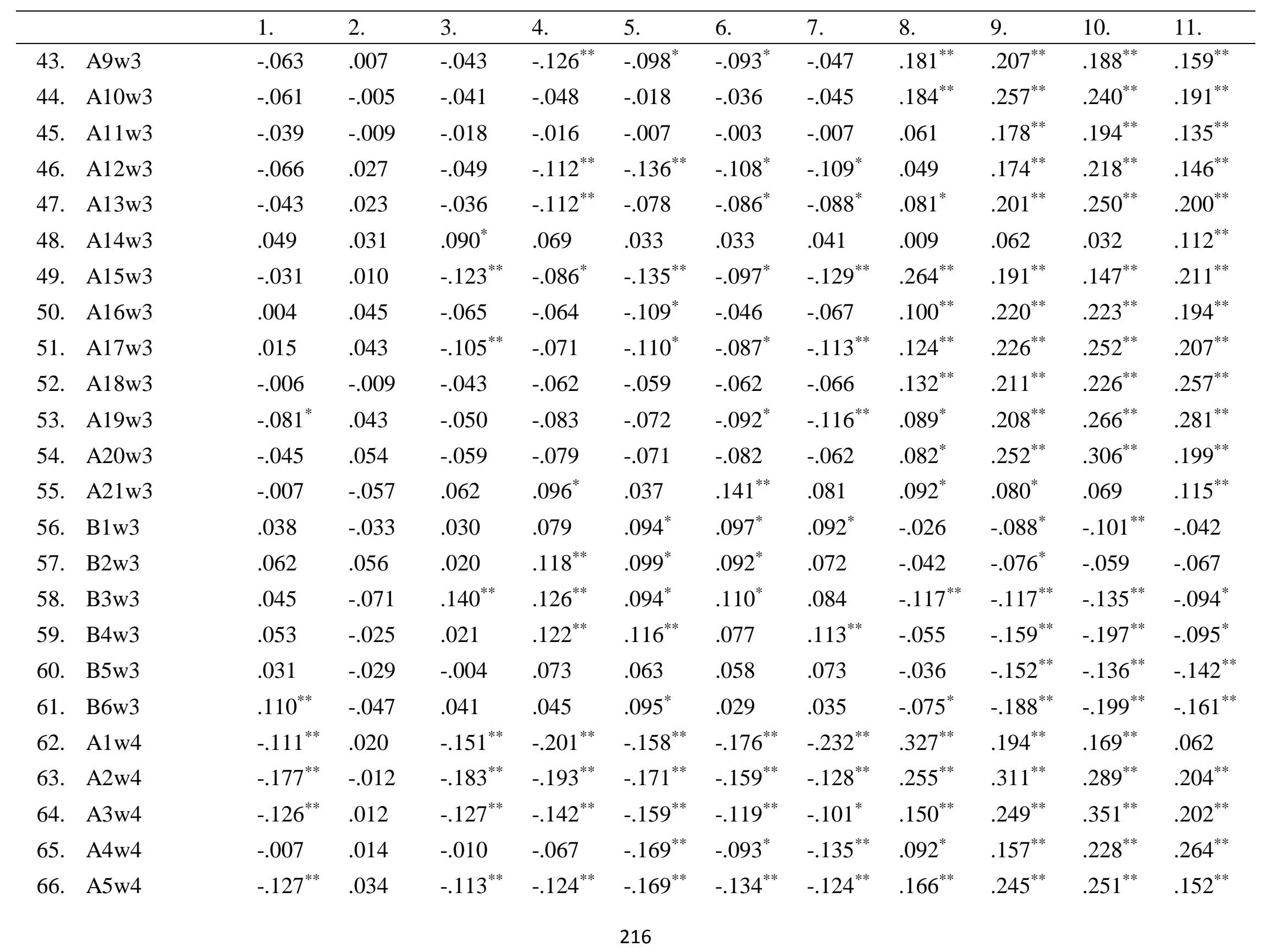




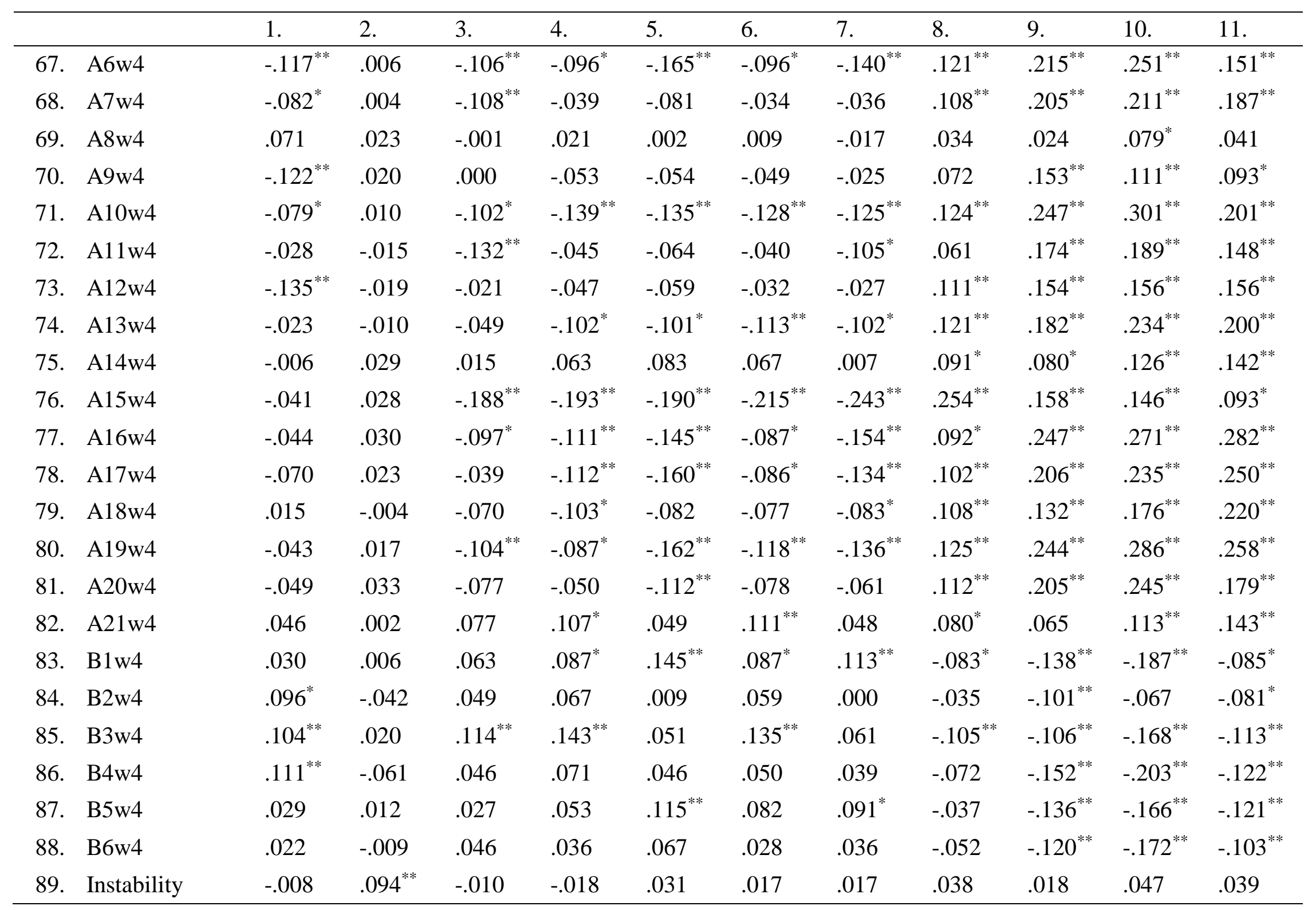




\begin{tabular}{|c|c|c|c|c|c|c|c|c|c|c|c|}
\hline & 12. & 13. & 14. & 15. & 16. & 17. & 18. & 19. & 20. & 21. & 22. \\
\hline 12. A5w1 & 1 & & & & & & & & & & \\
\hline 13. A6w1 & $.643^{* *}$ & 1 & & & & & & & & & \\
\hline 14. $\mathrm{A} 7 \mathrm{w} 1$ & $.422^{* *}$ & $.470^{* *}$ & 1 & & & & & & & & \\
\hline 15. A8w1 & $.091^{* * *}$ & $.104^{* *}$ & $.106^{* *}$ & 1 & & & & & & & \\
\hline 16. A9w1 & $.313^{* *}$ & $.360^{* *}$ & $.358^{* *}$ & .049 & 1 & & & & & & \\
\hline 17. A10w1 & $.306^{* *}$ & $.347^{* *}$ & $.329^{* *}$ & $.185^{* *}$ & $.248^{* *}$ & 1 & & & & & \\
\hline 18. A11w1 & $.271^{* * *}$ & $.250^{* *}$ & $.229^{* *}$ & $.249^{* *}$ & $.178^{* *}$ & $.417^{* *}$ & 1 & & & & \\
\hline 19. A12w1 & $.309^{* * *}$ & $.309^{* *}$ & $.332^{* *}$ & .022 & $.384^{* *}$ & $.256^{* *}$ & $.187^{* *}$ & 1 & & & \\
\hline 20. A13w1 & $.364^{* *}$ & $.372^{* *}$ & $.433^{* *}$ & $.116^{* *}$ & $.358^{* *}$ & $.329^{* *}$ & $.291^{* *}$ & $.311^{* *}$ & 1 & & \\
\hline 21. A14w1 & $.080^{*}$ & $.112^{* *}$ & $.126^{* *}$ & $.260^{* *}$ & $.101^{* *}$ & $.154^{* *}$ & $.269^{* *}$ & $.100^{* *}$ & $.145^{* *}$ & 1 & \\
\hline 22. A15w1 & $.268^{* *}$ & $.273^{* *}$ & $.276^{* *}$ & $.159^{* *}$ & $.191^{* *}$ & $.310^{* *}$ & $.274^{* *}$ & $.213^{* *}$ & $.236^{* *}$ & $.110^{* *}$ & 1 \\
\hline 23. A16w1 & $.377^{* *}$ & $.392^{* *}$ & $.491^{* *}$ & $.128^{* *}$ & $.340^{* *}$ & $.336^{* *}$ & $.296^{* *}$ & $.315^{* *}$ & $.440^{* *}$ & $.171^{* *}$ & $.280^{* *}$ \\
\hline 24. A17w1 & $.362^{* *}$ & $.357^{* *}$ & $.370^{* *}$ & $.170^{* *}$ & $.321^{* *}$ & $.360^{* *}$ & $.402^{* *}$ & $.333^{* *}$ & $.406^{* *}$ & $.228^{* *}$ & $.373^{* *}$ \\
\hline 25. A18w1 & $.265^{* *}$ & $.277^{* *}$ & $.340^{* *}$ & $.163^{* *}$ & $.244^{* *}$ & $.268^{* *}$ & $.320^{* *}$ & $.230^{* *}$ & $.408^{* *}$ & $.255^{* *}$ & $.260^{* *}$ \\
\hline 26. A19w1 & $.363^{* *}$ & $.361^{* *}$ & $.486^{* *}$ & $.097^{* *}$ & $.354^{* *}$ & $.325^{* *}$ & $.276^{* *}$ & $.345^{* *}$ & $.551^{* *}$ & $.156^{* *}$ & $.170^{* *}$ \\
\hline 27. A20w1 & $.387^{* *}$ & $.374^{* *}$ & $.399^{* *}$ & $.177^{* *}$ & $.345^{* *}$ & $.321^{* *}$ & $.282^{* *}$ & $.253^{* *}$ & $.505^{* *}$ & $.162^{* *}$ & $.254^{* *}$ \\
\hline 28. A21w1 & $.153^{* * *}$ & $.090^{* *}$ & $.165^{* *}$ & $.230^{* *}$ & .048 & $.295^{* * *}$ & $.271^{* *}$ & $.119^{* * *}$ & $.144^{* *}$ & $.252^{* *}$ & $.171^{* *}$ \\
\hline 29. B1w1 & $-.166^{* *}$ & $-.182^{* *}$ & $-.251^{* *}$ & -.009 & $-.142^{* *}$ & $-.127^{* *}$ & $-.114^{* *}$ & $-.137^{* *}$ & $-.270^{* *}$ & -.035 & $-.134^{* *}$ \\
\hline 30. B2w1 & $-.137^{* *}$ & $-.162^{* *}$ & $-.189^{* *}$ & .066 & $-.148^{* *}$ & $-.117^{* *}$ & -.045 & $-.192^{* *}$ & $-.174^{* *}$ & .034 & $-.084^{*}$ \\
\hline 31. B3w1 & $-.210^{* *}$ & $-.193^{* *}$ & $-.186^{* *}$ & .059 & $-.193^{* *}$ & $-.109^{* *}$ & -.019 & $-.104^{* *}$ & $-.143^{* *}$ & $.073^{*}$ & $-.118^{* *}$ \\
\hline 32. B4w1 & $-.210^{* *}$ & $-.291^{* *}$ & $-.212^{* *}$ & -.020 & $-.259^{* *}$ & $-.161^{* *}$ & $-.099^{* *}$ & $-.212^{* *}$ & $-.256^{* *}$ & -.002 & $-.144^{* *}$ \\
\hline 33. B5w1 & $-.163^{* *}$ & $-.163^{* *}$ & $-.242^{* *}$ & -.016 & $-.147^{* *}$ & $-.111^{* *}$ & $-.084^{*}$ & $-.168^{* *}$ & $-.290^{* *}$ & .034 & -.068 \\
\hline 34. B6w1 & $-.246^{* *}$ & $-.226^{* *}$ & $-.326^{* *}$ & -.065 & $-.213^{* *}$ & $-.180^{* *}$ & $-.118^{* *}$ & $-.226^{* *}$ & $-.350^{* *}$ & -.047 & $-.149^{* *}$ \\
\hline 35. A1w3 & $.132^{* * *}$ & $.176^{* *}$ & $.145^{* *}$ & -.001 & $.160^{* *}$ & $.193^{* *}$ & $.085^{*}$ & $.163^{* *}$ & $.118^{* *}$ & .024 & $.183^{* *}$ \\
\hline
\end{tabular}




\begin{tabular}{|c|c|c|c|c|c|c|c|c|c|c|c|c|}
\hline & & 12. & 13. & 14. & 15. & 16. & 17. & 18. & 19. & 20. & 21. & 22. \\
\hline 36. & A2w3 & $.318^{* *}$ & $.297^{* *}$ & $.243^{* *}$ & .066 & $.215^{* *}$ & $.237^{* *}$ & $.221^{* *}$ & $.123^{* *}$ & $.227^{* *}$ & .022 & $.134^{* * *}$ \\
\hline 37. & A3w3 & $.296^{* *}$ & $.290^{* *}$ & $.234^{* *}$ & .040 & $.187^{* *}$ & $.272^{* * *}$ & $.236^{* *}$ & $.125^{* *}$ & $.190^{* *}$ & .005 & $.167^{* *}$ \\
\hline 38. & A4w3 & $.184^{* *}$ & $.204^{* *}$ & $.214^{* *}$ & .030 & $.180^{* *}$ & $.198^{* *}$ & $.155^{* *}$ & $.174^{* *}$ & $.252^{* *}$ & .070 & $.151^{* *}$ \\
\hline 39. & A5w3 & $.454^{* *}$ & $.417^{* *}$ & $.301^{* *}$ & -.008 & $.273^{* *}$ & $.247^{* * *}$ & $.202^{* *}$ & $.228^{* *}$ & $.251^{* *}$ & .066 & $.212^{* *}$ \\
\hline 40. & A6w3 & $.344^{* *}$ & $.428^{* *}$ & $.325^{* *}$ & .035 & $.275^{* *}$ & $.250^{* *}$ & $.185^{* *}$ & $.278^{* *}$ & $.250^{* *}$ & .043 & $.176^{* *}$ \\
\hline 41. & A7w3 & $.311^{* *}$ & $.298^{* *}$ & $.368^{* *}$ & .044 & $.201^{* *}$ & $.240^{* *}$ & $.159^{* *}$ & $.209^{* *}$ & $.225^{* *}$ & .006 & $.115^{* *}$ \\
\hline 42. & A8w3 & .028 & .047 & $.100^{* *}$ & $.232^{* *}$ & .007 & $.126^{* *}$ & $.139^{* *}$ & .025 & .022 & $.128^{* *}$ & .053 \\
\hline 43. & A9w3 & $.237^{* *}$ & $.281^{* *}$ & $.222^{* *}$ & .045 & $.371^{* *}$ & $.208^{* *}$ & $.203^{* *}$ & $.227^{* *}$ & $.250^{* *}$ & $.096^{*}$ & $.185^{* *}$ \\
\hline 44. & A10w3 & $.287^{* *}$ & $.331^{* *}$ & $.287^{* *}$ & $.110^{* *}$ & $.223^{* *}$ & $.353^{* *}$ & $.270^{* *}$ & $.154^{* *}$ & $.240^{* *}$ & $.093^{*}$ & $.225^{* *}$ \\
\hline 45. & A11w3 & $.190^{* *}$ & $.176^{* *}$ & $.174^{* *}$ & $.087^{*}$ & $.172^{* *}$ & $.210^{* *}$ & $.264^{* *}$ & $.117^{* *}$ & $.154^{* *}$ & $.122^{* *}$ & $.181^{* *}$ \\
\hline 46. & A12w3 & $.217^{* *}$ & $.277^{* *}$ & $.281^{* *}$ & .055 & $.383^{* *}$ & $.240^{* *}$ & $.178^{* *}$ & $.323^{* *}$ & $.238^{* *}$ & .016 & $.141^{* *}$ \\
\hline 47. & A13w3 & $.212^{* *}$ & $.263^{* *}$ & $.208^{* *}$ & .043 & $.265^{* *}$ & $.229^{* *}$ & $.177^{* *}$ & $.180^{* *}$ & $.379^{* *}$ & $.081^{*}$ & $.190^{* *}$ \\
\hline 48. & A14w3 & $.094^{*}$ & $.074^{*}$ & $.084^{*}$ & $.100^{* *}$ & $.112^{* *}$ & $.119^{* *}$ & $.184^{* *}$ & $.078^{*}$ & $.117^{* *}$ & $.279^{* *}$ & $.085^{*}$ \\
\hline 49. & A15w3 & $.232^{* *}$ & $.237^{* *}$ & $.163^{* *}$ & .028 & $.179^{* *}$ & $.146^{* * *}$ & $.149^{* *}$ & $.147^{* *}$ & $.128^{* * *}$ & .067 & $.296^{* * *}$ \\
\hline 50. & A16w3 & $.257^{* *}$ & $.274^{* *}$ & $.257^{* *}$ & .022 & $.243^{* *}$ & $.234^{* *}$ & $.171^{* *}$ & $.203^{* *}$ & $.195^{* *}$ & .066 & $.132^{* *}$ \\
\hline 51. & A17w3 & $.261^{* *}$ & $.286^{* *}$ & $.218^{* *}$ & $.083^{*}$ & $.229^{* *}$ & $.239^{* * *}$ & $.263^{* *}$ & $.186^{* *}$ & $.185^{* *}$ & $.082^{*}$ & $.253^{* *}$ \\
\hline 52. & A18w3 & $.236^{* *}$ & $.268^{* *}$ & $.257^{* *}$ & $.081^{*}$ & $.226^{* *}$ & $.230^{* *}$ & $.256^{* *}$ & $.199^{* *}$ & $.248^{* *}$ & $.111^{* *}$ & $.234^{* *}$ \\
\hline 53. & A19w3 & $.286^{* *}$ & $.317^{* *}$ & $.299^{* *}$ & .038 & $.289^{* *}$ & $.245^{* *}$ & $.232^{* *}$ & $.244^{* *}$ & $.327^{* *}$ & .059 & $.146^{* *}$ \\
\hline 54. & A20w3 & $.234^{* *}$ & $.217^{* *}$ & $.272^{* *}$ & $.086^{*}$ & $.248^{* *}$ & $.225^{* *}$ & $.212^{* *}$ & $.159^{* *}$ & $.293^{* *}$ & .063 & $.149^{* *}$ \\
\hline 55. & A21w3 & $.084^{*}$ & .053 & $.138^{* * *}$ & $.095^{*}$ & .058 & $.152^{* *}$ & $.145^{* *}$ & $.129^{* * *}$ & $.108^{* * *}$ & $.192^{* * *}$ & .055 \\
\hline 56. & B1w3 & $-.128^{* *}$ & $-.125^{* *}$ & $-.118^{* *}$ & -.029 & $-.222^{* *}$ & -.029 & -.017 & $-.107^{* *}$ & $-.157^{* *}$ & .060 & $-.079^{*}$ \\
\hline 57. & B2w3 & -.044 & -.062 & $-.092^{*}$ & .025 & $-.147^{* *}$ & -.035 & .050 & $-.150^{* *}$ & $-.125^{* *}$ & .060 & -.016 \\
\hline 58. & B3w3 & $-.177^{* *}$ & $-.157^{* *}$ & $-.147^{* *}$ & -.021 & $-.167^{* *}$ & $-.131^{* *}$ & -.049 & $-.131^{* *}$ & $-.134^{* *}$ & .050 & $-.130^{* *}$ \\
\hline 59. & B4w3 & $-.198^{* * *}$ & $-.194^{* *}$ & $-.186^{* *}$ & -.073 & $-.241^{* *}$ & $-.154^{* *}$ & $-.084^{*}$ & $-.207^{* *}$ & $-.204^{* *}$ & -.013 & $-.134^{* *}$ \\
\hline
\end{tabular}




\begin{tabular}{|c|c|c|c|c|c|c|c|c|c|c|c|}
\hline & 12. & 13. & 14. & 15. & 16. & 17. & 18. & 19. & 20. & 21. & 22. \\
\hline 60. B5w3 & $-.153^{* *}$ & $-.139^{* *}$ & $-.174^{* *}$ & -.017 & $-.166^{* *}$ & $-.094^{*}$ & -.049 & $-.110^{* * *}$ & $-.154^{* * *}$ & .035 & -.033 \\
\hline 61. B6w3 & $-.247^{* *}$ & $-.241^{* *}$ & $-.255^{* *}$ & $-.084^{*}$ & $-.209^{* *}$ & $-.149^{* *}$ & $-.109^{* *}$ & $-.154^{* *}$ & $-.198^{* *}$ & -.023 & $-.121^{* *}$ \\
\hline 62. A1w4 & $.168^{* *}$ & $.180^{* *}$ & $.130^{* *}$ & .036 & $.107^{* *}$ & $.162^{* *}$ & .058 & $.076^{*}$ & $.128^{* *}$ & .004 & $.198^{* *}$ \\
\hline 63. $\mathrm{A} 2 \mathrm{w} 4$ & $.305^{* *}$ & $.269^{* * *}$ & $.255^{* *}$ & .038 & $.195^{* *}$ & $.167^{* *}$ & $.101^{* *}$ & $.110^{* *}$ & $.228^{* *}$ & -.015 & $.154^{* *}$ \\
\hline 64. $\mathrm{A} 3 \mathrm{w} 4$ & $.305^{* *}$ & $.221^{* *}$ & $.203^{* *}$ & .002 & $.164^{* *}$ & $.193^{* *}$ & $.124^{* *}$ & $.111^{* *}$ & $.223^{* *}$ & -.069 & $.145^{* *}$ \\
\hline 65. A4w4 & $.209^{* *}$ & $.176^{* *}$ & $.175^{* *}$ & .002 & $.185^{* *}$ & $.191^{* *}$ & $.082^{*}$ & $.122^{* *}$ & $.247^{* *}$ & .033 & $.173^{* *}$ \\
\hline 66. A5w4 & $.400^{* *}$ & $.324^{* *}$ & $.231^{* *}$ & .001 & $.180^{* * *}$ & $.196^{* *}$ & $.075^{*}$ & $.148^{* *}$ & $.225^{* *}$ & -.058 & $.179^{* * *}$ \\
\hline 67. A6w4 & $.312^{* *}$ & $.312^{* *}$ & $.215^{* *}$ & -.028 & $.219^{* *}$ & $.161^{* *}$ & $.109^{* *}$ & $.176^{* *}$ & $.159^{* *}$ & -.035 & $.137^{* *}$ \\
\hline 68. A7w4 & $.271^{* * *}$ & $.210^{* * *}$ & $.341^{* *}$ & .001 & $.196^{* * *}$ & $.186^{* *}$ & $.087^{*}$ & $.213^{* *}$ & $.239^{* *}$ & .024 & $.142^{* *}$ \\
\hline 69. A8w4 & $.083^{*}$ & $.092^{*}$ & $.088^{*}$ & $.223^{* *}$ & .020 & $.100^{* *}$ & $.137^{* *}$ & -.040 & $.076^{*}$ & $.131^{* *}$ & .057 \\
\hline 70. A9w4 & $.202^{* *}$ & $.152^{* *}$ & $.184^{* *}$ & .020 & $.245^{* *}$ & $.100^{* *}$ & $.080^{*}$ & $.165^{* *}$ & $.166^{* *}$ & .025 & .039 \\
\hline 71. A10w4 & $.276^{* *}$ & $.246^{* *}$ & $.234^{* *}$ & .061 & $.158^{* *}$ & $.341^{* *}$ & $.238^{* *}$ & $.125^{* *}$ & $.231^{* *}$ & .070 & $.141^{* *}$ \\
\hline 72. A11w4 & $.181^{* *}$ & $.133^{* *}$ & $.148^{* *}$ & .073 & $.093^{*}$ & $.196^{* *}$ & $.259^{* *}$ & .055 & $.128^{* *}$ & $.107^{* *}$ & $.147^{* *}$ \\
\hline 73. A12w4 & $.231^{* *}$ & $.151^{* *}$ & $.216^{* *}$ & .025 & $.223^{* *}$ & $.193^{* *}$ & $.116^{* *}$ & $.264^{* *}$ & $.216^{* *}$ & .067 & $.088^{*}$ \\
\hline 74. A13w4 & $.169^{* *}$ & $.160^{* *}$ & $.220^{* *}$ & -.015 & $.244^{* *}$ & $.175^{* *}$ & $.118^{* *}$ & $.172^{* *}$ & $.354^{* *}$ & $.079^{*}$ & $.114^{* *}$ \\
\hline 75. A14w4 & $.152^{* *}$ & $.134^{* *}$ & $.157^{* *}$ & $.106^{* *}$ & $.091^{*}$ & $.167^{* *}$ & $.144^{* *}$ & .056 & $.130^{* *}$ & $.276^{* *}$ & $.085^{*}$ \\
\hline 76. A15w4 & $.184^{* *}$ & $.163^{* *}$ & $.169^{* *}$ & .028 & $.127^{* *}$ & $.144^{* *}$ & $.094^{*}$ & $.141^{* *}$ & $.076^{*}$ & .037 & $.299^{* *}$ \\
\hline 77. A16w4 & $.253^{* *}$ & $.214^{* *}$ & $.266^{* *}$ & .027 & $.181^{* *}$ & $.222^{* *}$ & $.155^{* *}$ & $.225^{* *}$ & $.246^{* *}$ & $.078^{*}$ & $.197^{* *}$ \\
\hline 78. A17w4 & $.281^{* *}$ & $.213^{* *}$ & $.252^{* *}$ & $.083^{*}$ & $.249^{* *}$ & $.202^{* *}$ & $.200^{* *}$ & $.171^{* *}$ & $.261^{* *}$ & $.110^{* *}$ & $.225^{* *}$ \\
\hline 79. A18w4 & $.221^{* *}$ & $.206^{* *}$ & $.186^{* *}$ & .061 & $.195^{* *}$ & $.181^{* *}$ & $.194^{* *}$ & $.148^{* *}$ & $.246^{* *}$ & $.122^{* *}$ & $.225^{* *}$ \\
\hline 80. A19w4 & $.289^{* *}$ & $.250^{* *}$ & $.311^{* *}$ & .038 & $.268^{* *}$ & $.243^{* *}$ & $.171^{* *}$ & $.237^{* *}$ & $.305^{* *}$ & .069 & $.165^{* *}$ \\
\hline 81. A20w4 & $.240^{* *}$ & $.200^{* *}$ & $.200^{* *}$ & .021 & $.212^{* *}$ & $.162^{* *}$ & $.178^{* *}$ & $.124^{* *}$ & $.273^{* *}$ & .046 & $.150^{* *}$ \\
\hline 82. A21w4 & $.172^{* *}$ & $.105^{* *}$ & $.166^{* *}$ & $.095^{*}$ & .032 & $.114^{* *}$ & .070 & $.077^{*}$ & $.144^{* *}$ & .064 & $.126^{* *}$ \\
\hline 83. B1w4 & $-.140^{* *}$ & $-.144^{* *}$ & $-.088^{*}$ & $-.092^{*}$ & $-.153^{* *}$ & $-.096^{*}$ & $-.150^{* *}$ & $-.128^{* *}$ & $-.147^{* *}$ & -.050 & $-.075^{*}$ \\
\hline
\end{tabular}




\begin{tabular}{|c|c|c|c|c|c|c|c|c|c|c|c|}
\hline & 12. & 13. & 14. & 15. & 16. & 17. & 18. & 19. & 20. & 21. & 22. \\
\hline 84. B2w4 & -.034 & -.033 & -.066 & -.043 & -.070 & -.013 & -.017 & -.032 & -.059 & .036 & -.026 \\
\hline 85. B3w4 & $-.143^{* *}$ & $-.125^{* *}$ & $-.157^{* *}$ & -.031 & $-.143^{* *}$ & $-.142^{* *}$ & -.020 & $-.152^{* *}$ & $-.103^{* *}$ & .030 & $-.117^{* *}$ \\
\hline 86. B4w4 & $-.207^{* *}$ & $-.195^{* *}$ & $-.223^{* *}$ & -.048 & $-.172^{* *}$ & $-.109^{* *}$ & $-.087^{*}$ & $-.113^{* *}$ & $-.202^{* *}$ & -.067 & -.057 \\
\hline 87. B5w4 & $-.134^{* *}$ & $-.127^{* *}$ & $-.193^{* *}$ & -.072 & $-.159^{* *}$ & $-.105^{* *}$ & $-.107^{* *}$ & $-.170^{* *}$ & $-.127^{* *}$ & $-.085^{*}$ & $-.113^{* *}$ \\
\hline 88. B6w4 & $-.142^{* *}$ & $-.142^{* *}$ & $-.168^{* *}$ & -.011 & $-.138^{* *}$ & $-.109^{* *}$ & $-.101^{* *}$ & $-.118^{* *}$ & $-.177^{* *}$ & -.062 & $-.099^{* *}$ \\
\hline 89. Instability & .041 & .054 & .043 & .027 & .062 & .018 & .013 & $.102^{* *}$ & $.071^{*}$ & .035 & .029 \\
\hline & 23. & 24. & 25. & 26. & 27. & 28. & 29. & 30. & 31. & 32. & 33. \\
\hline 23. A16w1 & 1 & & & & & & & & & & \\
\hline 24. A17w1 & $.452^{* *}$ & 1 & & & & & & & & & \\
\hline 25. A18w1 & $.464^{* *}$ & $.490^{* *}$ & 1 & & & & & & & & \\
\hline 26. A19w1 & $.529^{* *}$ & $.431^{* *}$ & $.454^{* *}$ & 1 & & & & & & & \\
\hline 27. A20w1 & $.399^{* *}$ & $.376^{* *}$ & $.315^{* *}$ & $.402^{* *}$ & 1 & & & & & & \\
\hline 28. A21w1 & $.186^{* *}$ & $.310^{* *}$ & $.228^{* *}$ & $.196^{* *}$ & $.175^{* *}$ & 1 & & & & & \\
\hline 29. B1w1 & $-.211^{* *}$ & $-.193^{* *}$ & $-.133^{* *}$ & $-.224^{* *}$ & $-.228^{* *}$ & -.026 & 1 & & & & \\
\hline 30. $\mathrm{B} 2 \mathrm{w} 1$ & $-.139^{* *}$ & $-.165^{* *}$ & $-.113^{* *}$ & $-.145^{* *}$ & $-.117^{* *}$ & .059 & $.194^{* *}$ & 1 & & & \\
\hline 31. $\mathrm{B} 3 \mathrm{w} 1$ & $-.131^{* *}$ & $-.128^{* *}$ & -.054 & $-.141^{* *}$ & $-.121^{* *}$ & .006 & $.187^{* *}$ & $.269^{* *}$ & 1 & & \\
\hline 32. $\mathrm{B} 4 \mathrm{w} 1$ & $-.190^{* *}$ & $-.244^{* *}$ & $-.167^{* *}$ & $-.302^{* *}$ & $-.142^{* *}$ & -.032 & $.184^{* *}$ & $.238^{* *}$ & $.295^{* *}$ & 1 & \\
\hline 33. B5w1 & $-.170^{* *}$ & $-.160^{* *}$ & $-.116^{* *}$ & $-.315^{* *}$ & $-.205^{* *}$ & -.050 & $.272^{* *}$ & $.184^{* *}$ & $.237^{* *}$ & $.292^{* *}$ & 1 \\
\hline 34. B6w1 & $-.230^{* *}$ & $-.249^{* *}$ & $-.213^{* *}$ & $-.429^{* *}$ & $-.234^{* *}$ & $-.105^{* *}$ & $.260^{* *}$ & $.234^{* *}$ & $.275^{* *}$ & $.439^{* * *}$ & $.449^{* *}$ \\
\hline 35. A1w3 & $.136^{* *}$ & $.111^{* *}$ & $.128^{* *}$ & $.121^{* *}$ & $.108^{* *}$ & .054 & $-.165^{* *}$ & -.069 & $-.197^{* *}$ & $-.144^{* *}$ & $-.075^{*}$ \\
\hline 36. A2w3 & $.251^{* *}$ & $.209^{* *}$ & $.167^{* *}$ & $.264^{* *}$ & $.219^{* *}$ & .038 & $-.190^{* *}$ & -.059 & $-.153^{* *}$ & $-.162^{* *}$ & -.071 \\
\hline 37. $\mathrm{A} 3 \mathrm{w} 3$ & $.224^{* *}$ & $.249^{* *}$ & $.178^{* *}$ & $.220^{* *}$ & $.231^{* *}$ & .046 & $-.215^{* *}$ & -.066 & $-.117^{* *}$ & $-.152^{* *}$ & $-.089^{*}$ \\
\hline 38. A4w3 & $.272^{* *}$ & $.210^{* *}$ & $.214^{* *}$ & $.270^{* *}$ & $.226^{* *}$ & $.104^{* *}$ & $-.131^{* *}$ & -.021 & $-.121^{* *}$ & $-.147^{* *}$ & $-.086^{*}$ \\
\hline
\end{tabular}




\begin{tabular}{|c|c|c|c|c|c|c|c|c|c|c|c|c|}
\hline & & 23. & 24. & 25. & 26. & 27. & 28. & 29. & 30. & 31. & 32. & 33. \\
\hline 39. & A5w3 & $.267^{* * *}$ & $.266^{* * *}$ & $.214^{* * *}$ & $.320^{* *}$ & $.300^{* * *}$ & $.143^{* *}$ & $-.187^{* *}$ & $-.111^{* *}$ & $-.153^{* *}$ & $-.172^{* *}$ & $-.154^{* *}$ \\
\hline 40. & A6w3 & $.291^{* * *}$ & $.239^{* * *}$ & $.217^{* *}$ & $.316^{* *}$ & $.261^{* *}$ & .050 & $-.191^{* *}$ & $-.164^{* *}$ & $-.152^{* *}$ & $-.215^{* *}$ & $-.141^{* *}$ \\
\hline 41. & A7w3 & $.269^{* *}$ & $.204^{* *}$ & $.213^{* *}$ & $.339^{* *}$ & $.215^{* *}$ & $.075^{*}$ & $-.217^{* *}$ & $-.128^{* *}$ & $-.154^{* *}$ & $-.184^{* *}$ & $-.185^{* *}$ \\
\hline 42. & A8w3 & $.080^{*}$ & $.082^{*}$ & $.108^{* *}$ & $.118^{* *}$ & .066 & $.110^{* *}$ & -.050 & $.083^{*}$ & .060 & .055 & -.002 \\
\hline & A9w3 & $.214^{* *}$ & $.271^{* *}$ & $.202^{* *}$ & $.263^{* *}$ & $.249^{* *}$ & .067 & $-.186^{* *}$ & $-.102^{* *}$ & $-.121^{* *}$ & $-.239^{* *}$ & $-.096^{*}$ \\
\hline 44. & A10w3 & $.282^{* *}$ & $.279^{* *}$ & $.197^{* *}$ & $.250^{* *}$ & $.227^{* *}$ & $.142^{* *}$ & $-.203^{* *}$ & -.063 & $-.082^{*}$ & $-.205^{* *}$ & -.045 \\
\hline 45. & A11w3 & $.178^{* *}$ & $.269^{* *}$ & $.172^{* *}$ & $.215^{* *}$ & $.195^{* *}$ & $.144^{* *}$ & $-.147^{* *}$ & -.032 & -.005 & $-.147^{* *}$ & $-.079^{*}$ \\
\hline 46. & A12w3 & $.215^{* *}$ & $.229^{* *}$ & $.197^{* *}$ & $.287^{* *}$ & $.226^{* *}$ & $.086^{*}$ & $-.167^{* *}$ & $-.113^{* *}$ & $-.105^{* *}$ & $-.201^{* *}$ & $-.146^{* *}$ \\
\hline 47. & A13w3 & $.241^{* *}$ & $.200^{* *}$ & $.229^{* *}$ & $.324^{* *}$ & $.280^{* *}$ & .031 & $-.186^{* *}$ & $-.137^{* *}$ & $-.125^{* *}$ & $-.223^{* *}$ & $-.184^{* *}$ \\
\hline 48. & A14w3 & $.121^{* *}$ & $.162^{* *}$ & $.147^{* *}$ & $.175^{* *}$ & $.166^{* *}$ & $.122^{* *}$ & $-.096^{*}$ & -.001 & $.097^{*}$ & -.062 & -.028 \\
\hline 49. & A15w3 & $.166^{* *}$ & $.166^{* *}$ & $.214^{* *}$ & $.194^{* *}$ & $.132^{* *}$ & $.104^{* *}$ & $-.165^{* *}$ & -.040 & $-.114^{* *}$ & $-.158^{* *}$ & $-.096^{*}$ \\
\hline 50. & A16w3 & $.364^{* *}$ & $.233^{* *}$ & $.203^{* *}$ & $.381^{* *}$ & $.224^{* *}$ & $.087^{*}$ & $-.173^{* *}$ & $-.126^{* *}$ & $-.146^{* *}$ & $-.193^{* *}$ & $-.179^{* *}$ \\
\hline & A17w3 & $.344^{* *}$ & $.339^{* *}$ & $.257^{* *}$ & $.247^{* *}$ & $.243^{* *}$ & $.163^{* *}$ & $-.201^{* *}$ & $-.078^{*}$ & $-.118^{* *}$ & $-.117^{* *}$ & -.035 \\
\hline & A18w3 & $.278^{* *}$ & $.279^{* *}$ & $.383^{* *}$ & $.325^{* *}$ & $.260^{* *}$ & $.118^{* *}$ & $-.120^{* *}$ & $-.110^{* *}$ & $-.095^{*}$ & $-.207^{* *}$ & $-.109^{* *}$ \\
\hline & A19w3 & $.332^{* *}$ & $.281^{* *}$ & $.257^{* *}$ & $.450^{* *}$ & $.290^{* *}$ & $.133^{* *}$ & $-.169^{* *}$ & $-.131^{* *}$ & $-.152^{* *}$ & $-.219^{* *}$ & $-.218^{* * *}$ \\
\hline 54. & A20w3 & $.272^{* *}$ & $.246^{* *}$ & $.214^{* *}$ & $.254^{* *}$ & $.348^{* *}$ & $.077^{*}$ & $-.087^{*}$ & -.048 & $-.091^{*}$ & $-.140^{* *}$ & $-.122^{* *}$ \\
\hline & A21w3 & $.208^{* *}$ & $.185^{* *}$ & $.129^{* *}$ & $.185^{* *}$ & $.117^{* * *}$ & $.319^{* *}$ & $-.116^{* *}$ & .051 & .018 & $-.076^{*}$ & -.055 \\
\hline 56. & B1w3 & $-.098^{* *}$ & $-.083^{*}$ & $-.083^{*}$ & $-.178^{* *}$ & $-.167^{* *}$ & .018 & $.147^{* *}$ & .067 & $.082^{*}$ & $.097^{*}$ & $.090^{*}$ \\
\hline & $\mathrm{B} 2 \mathrm{w} 3$ & -.003 & -.071 & -.019 & $-.126^{* *}$ & $-.078^{*}$ & -.055 & $.107^{* *}$ & $.166^{* *}$ & $.151^{* *}$ & $.167^{* *}$ & $.187^{* *}$ \\
\hline 58. & B3w3 & $-.113^{* *}$ & $-.103^{* *}$ & $-.091^{*}$ & $-.121^{* *}$ & $-.136^{* *}$ & .010 & .062 & $.123^{* *}$ & $.254^{* * *}$ & $.153^{* *}$ & $.143^{* *}$ \\
\hline & B4w3 & $-.149^{* *}$ & $-.215^{* *}$ & $-.120^{* *}$ & $-.240^{* *}$ & $-.190^{* *}$ & -.072 & $.169^{* *}$ & $.187^{* *}$ & $.163^{* *}$ & $.272^{* *}$ & $.165^{* *}$ \\
\hline 60. & B5w3 & $-.160^{* *}$ & $-.116^{* *}$ & $-.161^{* *}$ & $-.201^{* *}$ & $-.160^{* *}$ & -.038 & $.109^{* *}$ & $.141^{* *}$ & $.184^{* *}$ & $.166^{* *}$ & $.208^{* *}$ \\
\hline 61. & B6w3 & $-.189^{* *}$ & $-.199^{* *}$ & $-.169^{* *}$ & $-.328^{* *}$ & $-.185^{* *}$ & $-.083^{*}$ & $.166^{* *}$ & $.116^{* *}$ & $.140^{* * *}$ & $.190^{* *}$ & $.166^{* *}$ \\
\hline 62. & A1w4 & $.112^{* *}$ & $.128^{* *}$ & .071 & $.085^{*}$ & $.148^{* *}$ & -.017 & $-.080^{*}$ & $-.089^{*}$ & $-.120^{* *}$ & $-.082^{*}$ & -.024 \\
\hline
\end{tabular}




\begin{tabular}{|c|c|c|c|c|c|c|c|c|c|c|c|}
\hline & 23. & 24. & 25. & 26. & 27. & 28. & 29. & 30. & 31. & 32. & 33. \\
\hline 63. A2w4 & $.229^{* *}$ & $.111^{* *}$ & $.128^{* * *}$ & $.261^{* *}$ & $.212^{* * *}$ & -.063 & $-.202^{* * *}$ & $-.131^{* * *}$ & $-.141^{* * *}$ & $-.198^{* * *}$ & $-.076^{*}$ \\
\hline 64. A3w4 & $.203^{* *}$ & $.154^{* *}$ & $.147^{* *}$ & $.227^{* *}$ & $.263^{* *}$ & -.029 & $-.146^{* *}$ & $-.093^{*}$ & $-.138^{* *}$ & $-.168^{* *}$ & -.054 \\
\hline 65. A4w4 & $.199^{* *}$ & $.197^{* *}$ & $.154^{* *}$ & $.214^{* *}$ & $.236^{* *}$ & .009 & $-.104^{* *}$ & $-.079^{*}$ & $-.090^{*}$ & $-.143^{* *}$ & $-.097^{*}$ \\
\hline 66. A5w4 & $.243^{* *}$ & $.160^{* *}$ & $.133^{* *}$ & $.257^{* *}$ & $.230^{* *}$ & .052 & $-.139^{* *}$ & -.025 & $-.193^{* *}$ & $-.172^{* *}$ & $-.093^{*}$ \\
\hline 67. A6w4 & $.225^{* *}$ & $.168^{* * *}$ & $.113^{* *}$ & $.244^{* *}$ & $.196^{* *}$ & -.044 & $-.173^{* *}$ & $-.080^{*}$ & $-.199^{* * *}$ & $-.188^{* *}$ & $-.125^{* *}$ \\
\hline 68. $\mathrm{A} 7 \mathrm{w} 4$ & $.243^{* *}$ & $.144^{* *}$ & $.145^{* *}$ & $.316^{* *}$ & $.194^{* *}$ & .035 & $-.120^{* *}$ & $-.197^{* *}$ & $-.173^{* *}$ & $-.157^{* *}$ & $-.141^{* *}$ \\
\hline 69. A8w4 & $.080^{*}$ & $.075^{*}$ & $.120^{* *}$ & $.088^{*}$ & .072 & $.129^{* * *}$ & .020 & .024 & .023 & -.050 & -.053 \\
\hline 70. $\mathrm{A} 9 \mathrm{w} 4$ & $.181^{* * *}$ & $.165^{* *}$ & $.094^{*}$ & $.165^{* *}$ & $.142^{* * *}$ & -.027 & -.072 & $-.112^{* *}$ & $-.108^{* *}$ & $-.143^{* *}$ & $-.109^{* *}$ \\
\hline 71. A10w4 & $.233^{* *}$ & $.229^{* *}$ & $.178^{* *}$ & $.251^{* *}$ & $.230^{* *}$ & .061 & $-.143^{* *}$ & $-.079^{*}$ & $-.104^{* *}$ & $-.190^{* *}$ & $-.081^{*}$ \\
\hline 72. A11w4 & $.168^{* *}$ & $.165^{* *}$ & $.162^{* *}$ & $.138^{* *}$ & $.170^{* *}$ & .062 & $-.129^{* *}$ & -.063 & -.043 & $-.114^{* *}$ & -.073 \\
\hline 73. A12w4 & $.230^{* *}$ & $.161^{* *}$ & $.171^{* *}$ & $.215^{* *}$ & $.193^{* *}$ & .058 & $-.102^{* *}$ & $-.119^{* *}$ & $-.102^{* *}$ & $-.128^{* *}$ & $-.088^{*}$ \\
\hline 74. A13w4 & $.238^{* *}$ & $.163^{* *}$ & $.181^{* *}$ & $.236^{* *}$ & $.285^{* *}$ & .038 & $-.179^{* *}$ & $-.119^{* *}$ & $-.131^{* *}$ & $-.201^{* *}$ & $-.117^{* *}$ \\
\hline 75. A14w4 & $.196^{* *}$ & $.180^{* *}$ & $.180^{* *}$ & $.196^{* *}$ & $.130^{* *}$ & $.148^{* *}$ & $-.099^{* *}$ & -.072 & -.037 & $-.110^{* *}$ & -.073 \\
\hline 76. A15w4 & $.150^{* *}$ & $.128^{* * *}$ & $.103^{* *}$ & .062 & $.133^{* * *}$ & .058 & $-.103^{* *}$ & -.024 & -.068 & $-.089^{*}$ & -.050 \\
\hline 77. A16w4 & $.353^{* *}$ & $.229^{* *}$ & $.198^{* *}$ & $.349^{* *}$ & $.222^{* *}$ & $.078^{*}$ & $-.157^{* *}$ & $-.124^{* *}$ & $-.105^{* *}$ & $-.204^{* *}$ & $-.167^{* *}$ \\
\hline 78. A17w4 & $.266^{* *}$ & $.258^{* * *}$ & $.231^{* *}$ & $.298^{* *}$ & $.252^{* *}$ & .058 & $-.169^{* *}$ & $-.142^{* *}$ & $-.101^{* *}$ & $-.185^{* *}$ & $-.175^{* *}$ \\
\hline 79. A18w4 & $.230^{* *}$ & $.214^{* *}$ & $.274^{* *}$ & $.254^{* *}$ & $.208^{* *}$ & $.082^{*}$ & $-.169^{* *}$ & $-.118^{* *}$ & -.056 & $-.183^{* *}$ & $-.125^{* *}$ \\
\hline 80. A19w4 & $.333^{* *}$ & $.239^{* *}$ & $.214^{* *}$ & $.424^{* *}$ & $.270^{* * *}$ & $.096^{*}$ & $-.218^{* *}$ & $-.164^{* *}$ & $-.123^{* *}$ & $-.247^{* *}$ & $-.186^{* *}$ \\
\hline 81. A20w4 & $.244^{* *}$ & $.213^{* *}$ & $.195^{* *}$ & $.272^{* *}$ & $.308^{* *}$ & .012 & $-.150^{* *}$ & -.041 & $-.106^{* *}$ & $-.095^{*}$ & $-.130^{* *}$ \\
\hline 82. $\mathrm{A} 21 \mathrm{w} 4$ & $.142^{* *}$ & $.188^{* *}$ & $.115^{* *}$ & $.137^{* *}$ & $.130^{* * *}$ & $.189^{* *}$ & $-.120^{* *}$ & -.059 & -.030 & $-.079^{*}$ & -.066 \\
\hline 83. B1w4 & $-.110^{* *}$ & $-.087^{*}$ & -.048 & $-.144^{* *}$ & $-.142^{* *}$ & -.064 & $.187^{* *}$ & .013 & $.138^{* *}$ & $.159^{* *}$ & .056 \\
\hline 84. B2w4 & -.027 & -.015 & -.020 & $-.088^{*}$ & -.019 & .016 & $.077^{*}$ & $.176^{* *}$ & $.164^{* * *}$ & $.157^{* *}$ & $.132^{* *}$ \\
\hline 85. B3w4 & $-.106^{* *}$ & -.065 & $-.095^{*}$ & $-.134^{* *}$ & $-.176^{* *}$ & -.034 & $.173^{* *}$ & $.084^{*}$ & $.150^{* *}$ & $.188^{* *}$ & $.114^{* *}$ \\
\hline 86. B4w4 & $-.115^{* *}$ & $-.122^{* *}$ & $-.132^{* *}$ & $-.228^{* *}$ & $-.177^{* *}$ & -.002 & $.178^{* *}$ & $.144^{* *}$ & $.115^{* *}$ & $.231^{* *}$ & $.135^{* *}$ \\
\hline
\end{tabular}




\begin{tabular}{|c|c|c|c|c|c|c|c|c|c|c|c|}
\hline & 23. & 24. & 25. & 26. & 27. & 28. & 29. & 30. & 31. & 32. & 33. \\
\hline 87. B5w4 & $-.126^{* *}$ & $-.141^{* *}$ & $-.120^{* *}$ & $-.187^{* *}$ & $-.164^{* *}$ & -.046 & $.207^{* *}$ & $.111^{* * *}$ & $.130^{* * *}$ & $.135^{* *}$ & $.173^{* *}$ \\
\hline 88. B6w4 & $-.115^{* *}$ & $-.107^{* *}$ & $-.094^{*}$ & $-.259^{* *}$ & $-.134^{* *}$ & -.032 & $.207^{* *}$ & $.087^{*}$ & $.112^{* *}$ & $.213^{* *}$ & $.203^{* *}$ \\
\hline 89. Instability & .057 & .057 & .024 & $.083^{*}$ & .041 & .016 & -.065 & $-.072^{*}$ & -.030 & $-.073^{*}$ & -.064 \\
\hline & 34. & 35. & 36. & 37. & 38. & 39. & 40. & 41. & 42. & 43. & 44. \\
\hline 34. B6w1 & 1 & & & & & & & & & & \\
\hline 35. A1w3 & $-.140^{* *}$ & 1 & & & & & & & & & \\
\hline 36. A2w3 & $-.148^{* *}$ & $.333^{* *}$ & 1 & & & & & & & & \\
\hline 37. $\mathrm{A} 3 \mathrm{w} 3$ & $-.151^{* *}$ & $.253^{* *}$ & $.578^{* *}$ & 1 & & & & & & & \\
\hline 38. A4w3 & $-.163^{* *}$ & $.172^{* *}$ & $.349^{* *}$ & $.353^{* *}$ & 1 & & & & & & \\
\hline 39. A5w3 & $-.218^{* *}$ & $.257^{* *}$ & $.418^{* *}$ & $.421^{* *}$ & $.300^{* *}$ & 1 & & & & & \\
\hline 40. A6w3 & $-.231^{* *}$ & $.240^{* *}$ & $.398^{* *}$ & $.363^{* *}$ & $.309^{* *}$ & $.700^{* *}$ & 1 & & & & \\
\hline 41. $\mathrm{A} 7 \mathrm{w} 3$ & $-.237^{* *}$ & $.208^{* *}$ & $.436^{* *}$ & $.424^{* *}$ & $.346^{* *}$ & $.472^{* *}$ & $.481^{* *}$ & 1 & & & \\
\hline 42. A8w3 & .026 & .073 & $.130^{* *}$ & .061 & $.146^{* *}$ & $.117^{* *}$ & $.112^{* *}$ & $.178^{* *}$ & 1 & & \\
\hline 43. A9w3 & $-.160^{* *}$ & $.262^{* *}$ & $.294^{* *}$ & $.269^{* *}$ & $.318^{* *}$ & $.437^{* *}$ & $.451^{* *}$ & $.416^{* *}$ & $.102^{* *}$ & 1 & \\
\hline 44. A10w3 & $-.166^{* *}$ & $.338^{* *}$ & $.364^{* *}$ & $.406^{* *}$ & $.352^{* *}$ & $.406^{* *}$ & $.394^{* *}$ & $.396^{* *}$ & $.147^{* *}$ & $.390^{* *}$ & 1 \\
\hline 45. A11w3 & $-.122^{* *}$ & $.203^{* *}$ & $.250^{* *}$ & $.284^{* *}$ & $.272^{* *}$ & $.326^{* *}$ & $.332^{* *}$ & $.320^{* *}$ & $.246^{* *}$ & $.353^{* *}$ & $.415^{* *}$ \\
\hline 46. A12w3 & $-.182^{* *}$ & $.178^{* *}$ & $.271^{* *}$ & $.263^{* *}$ & $.284^{* *}$ & $.411^{* *}$ & $.495^{* *}$ & $.443^{* *}$ & $.139^{* *}$ & $.552^{* *}$ & $.330^{* *}$ \\
\hline 47. A13w3 & $-.207^{* *}$ & $.209^{* *}$ & $.310^{* *}$ & $.337^{* *}$ & $.330^{* *}$ & $.397^{* *}$ & $.401^{* *}$ & $.456^{* *}$ & $.081^{*}$ & $.478^{* *}$ & $.356^{* *}$ \\
\hline 48. A14w3 & -.043 & .051 & $.102^{* *}$ & $.113^{* *}$ & $.175^{* *}$ & $.180^{* *}$ & $.132^{* *}$ & $.159^{* *}$ & $.234^{* *}$ & $.178^{* *}$ & $.237^{* *}$ \\
\hline 49. A15w3 & $-.094^{*}$ & $.230^{* *}$ & $.267^{* *}$ & $.247^{* *}$ & $.256^{* *}$ & $.288^{* *}$ & $.219^{* *}$ & $.202^{* *}$ & $.081^{*}$ & $.224^{* *}$ & $.279^{* *}$ \\
\hline 50. A16w3 & $-.151^{* *}$ & $.210^{* *}$ & $.404^{* *}$ & $.366^{* *}$ & $.352^{* *}$ & $.410^{* *}$ & $.433^{* *}$ & $.493^{* *}$ & $.184^{* *}$ & $.364^{* *}$ & $.380^{* *}$ \\
\hline 51. A17w3 & $-.102^{* *}$ & $.192^{* *}$ & $.329^{* *}$ & $.345^{* *}$ & $.323^{* *}$ & $.407^{* *}$ & $.409^{* *}$ & $.373^{* *}$ & $.149^{* *}$ & $.345^{* *}$ & $.399^{* *}$ \\
\hline 52. A18w3 & $-.166^{* *}$ & $.210^{* *}$ & $.361^{* *}$ & $.336^{* *}$ & $.436^{* *}$ & $.402^{* *}$ & $.432^{* *}$ & $.410^{* *}$ & $.204^{* *}$ & $.366^{* *}$ & $.420^{* *}$ \\
\hline
\end{tabular}




\begin{tabular}{|c|c|c|c|c|c|c|c|c|c|c|c|c|}
\hline & & 34. & 35. & 36. & 37. & 38. & 39. & 40. & 41. & 42. & 43. & 44. \\
\hline 53. & A19w3 & $-.263^{* *}$ & $.181^{* *}$ & $.367^{* *}$ & $.377^{* *}$ & $.366^{* * *}$ & $.455^{* *}$ & $.453^{* *}$ & $.534^{* *}$ & $.120^{* *}$ & $.414^{* * *}$ & $.360^{* *}$ \\
\hline 54. & A20w3 & $-.205^{* *}$ & $.167^{* *}$ & $.352^{* * *}$ & $.392^{* *}$ & $.351^{* *}$ & $.332^{* * *}$ & $.381^{* *}$ & $.370^{* *}$ & $.091^{*}$ & $.316^{* *}$ & $.378^{* *}$ \\
\hline 55. & A21w3 & $-.095^{*}$ & $.101^{* *}$ & $.184^{* *}$ & $.126^{* *}$ & $.205^{* *}$ & $.171^{* *}$ & $.186^{* *}$ & $.240^{* *}$ & $.225^{* *}$ & $.194^{* *}$ & $.299^{* *}$ \\
\hline 56. & B1w3 & $.147^{* *}$ & $-.106^{* *}$ & $-.164^{* *}$ & $-.149^{* *}$ & $-.103^{* *}$ & $-.184^{* *}$ & $-.185^{* *}$ & $-.198^{* * *}$ & -.007 & $-.194^{* *}$ & $-.082^{*}$ \\
\hline 57. & $\mathrm{~B} 2 \mathrm{w} 3$ & $.146^{* *}$ & $-.100^{* *}$ & -.072 & -.042 & $-.150^{* * *}$ & $-.165^{* *}$ & $-.168^{* *}$ & $-.215^{* * *}$ & .018 & $-.220^{* *}$ & $-.103^{* *}$ \\
\hline 58. & B3w3 & $.167^{* *}$ & $-.155^{* *}$ & $-.237^{* *}$ & $-.208^{* *}$ & $-.151^{* * *}$ & $-.235^{* *}$ & $-.244^{* *}$ & $-.274^{* *}$ & .046 & $-.195^{* *}$ & $-.155^{* *}$ \\
\hline 59. & B4w3 & $.244^{* *}$ & $-.132^{* *}$ & $-.228^{* *}$ & $-.226^{* *}$ & $-.158^{* *}$ & $-.315^{* *}$ & $-.359^{* *}$ & $-.296^{* *}$ & .025 & $-.327^{* *}$ & $-.204^{* *}$ \\
\hline 60. & B5w3 & $.258^{* *}$ & $-.101^{* *}$ & $-.192^{* * *}$ & $-.190^{* *}$ & $-.177^{* *}$ & $-.229^{* *}$ & $-.256^{* *}$ & $-.304^{* *}$ & -.034 & $-.173^{* *}$ & $-.157^{* *}$ \\
\hline 61. & B6w3 & $.334^{* * *}$ & $-.155^{* *}$ & $-.261^{* *}$ & $-.310^{* *}$ & $-.213^{* *}$ & $-.325^{* *}$ & $-.338^{* *}$ & $-.371^{* *}$ & -.010 & $-.263^{* *}$ & $-.246^{* *}$ \\
\hline 62. & A1w4 & $-.097^{*}$ & $.435^{* *}$ & $.269^{* *}$ & $.168^{* *}$ & $.137^{* *}$ & $.184^{* *}$ & $.168^{* *}$ & $.149^{* * *}$ & .041 & $.124^{* *}$ & $.228^{* *}$ \\
\hline 63. & $\mathrm{~A} 2 \mathrm{w} 4$ & $-.182^{* * *}$ & $.287^{* *}$ & $.497^{* *}$ & $.381^{* *}$ & $.285^{* *}$ & $.313^{* *}$ & $.286^{* *}$ & $.311^{* *}$ & $.080^{*}$ & $.254^{* *}$ & $.296^{* *}$ \\
\hline 64. & $\mathrm{~A} 3 \mathrm{w} 4$ & $-.190^{* * *}$ & $.213^{* *}$ & $.376^{* *}$ & $.469^{* * *}$ & $.256^{* *}$ & $.337^{* * *}$ & $.276^{* *}$ & $.228^{* *}$ & .026 & $.220^{* * *}$ & $.246^{* *}$ \\
\hline 65. & A4w4 & $-.164^{* *}$ & $.155^{* *}$ & $.275^{* *}$ & $.251^{* *}$ & $.408^{* *}$ & $.212^{* *}$ & $.217^{* *}$ & $.204^{* *}$ & .038 & $.150^{* *}$ & $.266^{* *}$ \\
\hline 66. & $\mathrm{~A} 5 \mathrm{w} 4$ & $-.222^{* *}$ & $.208^{* *}$ & $.321^{* *}$ & $.253^{* *}$ & $.240^{* *}$ & $.471^{* *}$ & $.405^{* *}$ & $.329^{* *}$ & .072 & $.236^{* *}$ & $.289^{* *}$ \\
\hline 67. & A6w4 & $-.197^{* *}$ & $.211^{* *}$ & $.346^{* *}$ & $.266^{* *}$ & $.210^{* *}$ & $.463^{* *}$ & $.503^{* *}$ & $.322^{* *}$ & .026 & $.326^{* *}$ & $.263^{* *}$ \\
\hline 68. & $\mathrm{~A} 7 \mathrm{w} 4$ & $-.219^{* *}$ & $.185^{* *}$ & $.293^{* *}$ & $.242^{* *}$ & $.274^{* *}$ & $.373^{* *}$ & $.380^{* *}$ & $.491^{* *}$ & .065 & $.294^{* *}$ & $.248^{* *}$ \\
\hline 69. & A8w4 & -.049 & -.026 & $.138^{* *}$ & $.084^{*}$ & $.131^{* *}$ & $.108^{* *}$ & $.093^{*}$ & $.094^{*}$ & $.321^{* *}$ & .040 & $.091^{*}$ \\
\hline 70. & A9w4 & $-.132^{* *}$ & .067 & $.192^{* *}$ & $.136^{* *}$ & $.163^{* *}$ & $.255^{* *}$ & $.296^{* *}$ & $.275^{* *}$ & -.004 & $.347^{* *}$ & $.267^{* *}$ \\
\hline 71. & A10w4 & $-.151^{* *}$ & $.214^{* *}$ & $.329^{* *}$ & $.308^{* *}$ & $.302^{* *}$ & $.283^{* *}$ & $.290^{* *}$ & $.284^{* *}$ & $.095^{*}$ & $.237^{* *}$ & $.453^{* *}$ \\
\hline 72. & A11w4 & $-.115^{* *}$ & $.134^{* *}$ & $.247^{* *}$ & $.242^{* *}$ & $.201^{* *}$ & $.255^{* *}$ & $.240^{* *}$ & $.205^{* *}$ & $.134^{* *}$ & $.154^{* *}$ & $.283^{* *}$ \\
\hline 73. & A12w4 & $-.111^{* *}$ & $.117^{* *}$ & $.152^{* *}$ & $.121^{* *}$ & $.219^{* *}$ & $.307^{* *}$ & $.344^{* *}$ & $.292^{* *}$ & $.086^{*}$ & $.316^{* *}$ & $.230^{* *}$ \\
\hline 74. & A13w4 & $-.228^{* *}$ & $.193^{* *}$ & $.198^{* *}$ & $.165^{* *}$ & $.224^{* *}$ & $.271^{* *}$ & $.305^{* *}$ & $.239^{* *}$ & -.048 & $.254^{* *}$ & $.228^{* *}$ \\
\hline 75. & A14w4 & -.059 & .055 & $.094^{*}$ & $.112^{* *}$ & $.169^{* *}$ & $.113^{* *}$ & $.092^{*}$ & $.131^{* *}$ & $.121^{* *}$ & $.121^{* *}$ & $.125^{* *}$ \\
\hline 76. & A15w4 & -.008 & $.185^{* *}$ & $.165^{* *}$ & $.139^{* *}$ & $.149^{* *}$ & $.153^{* *}$ & $.116^{* *}$ & $.097^{*}$ & $.088^{*}$ & $.150^{* *}$ & $.193^{* *}$ \\
\hline
\end{tabular}




\begin{tabular}{|c|c|c|c|c|c|c|c|c|c|c|c|c|}
\hline & & 34. & 35. & 36. & 37. & 38. & 39. & 40. & 41. & 42. & 43. & 44. \\
\hline 77. & A16w4 & $-.213^{* *}$ & $.110^{* *}$ & $.266^{* *}$ & $.196^{* *}$ & $.283^{* * *}$ & $.265^{* *}$ & $.255^{* *}$ & $.314^{* *}$ & $.080^{*}$ & $.248^{* *}$ & $.277^{* *}$ \\
\hline 78. & $\mathrm{~A} 17 \mathrm{w} 4$ & $-.210^{* *}$ & $.197^{* *}$ & $.263^{* *}$ & $.230^{* *}$ & $.317^{* *}$ & $.337^{* *}$ & $.287^{* *}$ & $.264^{* *}$ & $.084^{*}$ & $.302^{* *}$ & $.298^{* *}$ \\
\hline 79. & A18w4 & $-.160^{* *}$ & $.125^{* *}$ & $.210^{* *}$ & $.176^{* *}$ & $.310^{* *}$ & $.275^{* *}$ & $.268^{* *}$ & $.229^{* *}$ & $.081^{*}$ & $.238^{* *}$ & $.256^{* *}$ \\
\hline 80. & A19w4 & $-.261^{* *}$ & $.134^{* *}$ & $.275^{* *}$ & $.238^{* *}$ & $.338^{* *}$ & $.294^{* *}$ & $.287^{* *}$ & $.372^{* *}$ & .065 & $.320^{* *}$ & $.316^{* *}$ \\
\hline & A20w4 & $-.166^{* *}$ & $.148^{* *}$ & $.184^{* *}$ & $.229^{* *}$ & $.190^{* * *}$ & $.217^{* *}$ & $.198^{* *}$ & $.200^{* *}$ & -.026 & $.202^{* *}$ & $.183^{* *}$ \\
\hline 82. & A21w4 & $-.138^{* *}$ & .036 & $.110^{* *}$ & $.092^{*}$ & $.204^{* *}$ & $.155^{* *}$ & $.094^{*}$ & $.146^{* *}$ & $.092^{*}$ & .061 & $.229^{* *}$ \\
\hline & B1w4 & $.143^{* *}$ & $-.131^{* *}$ & $-.218^{* *}$ & $-.161^{* *}$ & $-.186^{* *}$ & $-.149^{* * *}$ & $-.143^{* *}$ & $-.166^{* *}$ & -.054 & $-.181^{* *}$ & $-.134^{* *}$ \\
\hline & $\mathrm{B} 2 \mathrm{w} 4$ & $.098^{*}$ & $-.092^{*}$ & $-.142^{* *}$ & $-.121^{* *}$ & -.075 & $-.129^{* * *}$ & $-.145^{* *}$ & $-.101^{*}$ & .012 & $-.132^{* *}$ & $-.121^{* *}$ \\
\hline & B3w4 & $.165^{* *}$ & $-.174^{* *}$ & $-.197^{* *}$ & $-.159^{* *}$ & $-.106^{* *}$ & $-.179^{* *}$ & $-.172^{* *}$ & $-.189^{* *}$ & -.062 & $-.205^{* *}$ & $-.206^{* *}$ \\
\hline & B4w4 & $.233^{* *}$ & $-.141^{* *}$ & $-.255^{* *}$ & $-.211^{* *}$ & $-.211^{* *}$ & $-.345^{* *}$ & $-.318^{* *}$ & $-.297^{* *}$ & -.039 & $-.311^{* * *}$ & $-.199^{* *}$ \\
\hline & B5w4 & $.222^{* *}$ & -.047 & $-.108^{* *}$ & $-.096^{*}$ & $-.150^{* *}$ & $-.158^{* *}$ & $-.196^{* *}$ & $-.183^{* *}$ & -.037 & $-.171^{* *}$ & $-.122^{* *}$ \\
\hline 88. & B6w4 & $.302^{* *}$ & $-.121^{* *}$ & $-.147^{* *}$ & $-.164^{* *}$ & $-.175^{* *}$ & $-.236^{* *}$ & $-.263^{* *}$ & $-.268^{* *}$ & .001 & $-.233^{* *}$ & $-.175^{* *}$ \\
\hline 89. & Instability & -.060 & .053 & $.084^{*}$ & .014 & .007 & .030 & .049 & .003 & $-.075^{*}$ & .038 & .015 \\
\hline
\end{tabular}

45. A11w3 1

46. A12w3 . $356^{* *} 1$

47. A13w3 . $323^{* *} \quad .425^{* *} \quad 1$

48. A14w3 $.272^{* *} \quad .131^{* *} \quad .177^{* *} \quad 1$

49. A15w3 . $294^{* *} \quad .215^{* *} \quad .213^{* *} \quad .182^{* *} \quad 1$

50. A16w3 $\quad .402^{* *} \quad .343^{* *} \quad .418^{* *} \quad .240^{* *} \quad .277^{* *} \quad 1$

51. A17w3 $\quad .410^{* *} \quad .346^{* *} \quad .335^{* *} \quad .257^{* *} \quad .341^{* *} \quad .552^{* *} \quad 1$

52. A18w3 $\quad .344^{* *} \quad .363^{* *} \quad .444^{* *} \quad .222^{* *} \quad .281^{* *} \quad .484^{* *} \quad .481^{* *} \quad 1$

53. A19w3 $\quad .348^{* *} \quad .421^{* *} \quad .575^{* *} \quad .203^{* *} \quad .236^{* *} \quad .513^{* *} \quad .425^{* *} \quad .504^{* *} \quad 1$ 


\begin{tabular}{|c|c|c|c|c|c|c|c|c|c|c|c|}
\hline & 45. & 46. & 47. & 48. & 49. & 50. & 51. & 52. & 53. & 54. & 55. \\
\hline 54. A20w3 & $.333^{* *}$ & $.305^{* *}$ & $.477^{* *}$ & $.167^{* *}$ & $.285^{* *}$ & $.380^{* *}$ & $.361^{* *}$ & $.430^{* *}$ & $.453^{* *}$ & 1 & \\
\hline 55. A21w3 & $.271^{* *}$ & $.186^{* *}$ & $.151^{* *}$ & $.371^{* *}$ & $.178^{* *}$ & $.294^{* *}$ & $.358^{* *}$ & $.257^{* *}$ & $.242^{* *}$ & $.206^{* *}$ & 1 \\
\hline 56. B1w3 & $-.146^{* *}$ & $-.156^{* *}$ & $-.209^{* *}$ & -.020 & -.060 & $-.152^{* *}$ & $-.151^{* *}$ & $-.148^{* *}$ & $-.232^{* *}$ & $-.251^{* *}$ & .014 \\
\hline 57. $\mathrm{B} 2 \mathrm{w} 3$ & $-.123^{* *}$ & $-.199^{* *}$ & $-.185^{* *}$ & -.013 & -.060 & $-.141^{* *}$ & $-.100^{* *}$ & $-.173^{* *}$ & $-.229^{* *}$ & $-.124^{* *}$ & -.062 \\
\hline 58. B3w3 & -.069 & $-.180^{* *}$ & $-.228^{* *}$ & $.081^{*}$ & $-.084^{*}$ & $-.230^{* *}$ & $-.176^{* *}$ & $-.249^{* *}$ & $-.267^{* *}$ & $-.226^{* *}$ & .000 \\
\hline 59. B4w3 & $-.184^{* *}$ & $-.297^{* *}$ & $-.317^{* *}$ & $-.085^{*}$ & $-.176^{* *}$ & $-.258^{* *}$ & $-.249^{* *}$ & $-.294^{* *}$ & $-.376^{* *}$ & $-.201^{* *}$ & $-.103^{* *}$ \\
\hline 60. B5w3 & $-.156^{* *}$ & $-.253^{* *}$ & $-.231^{* *}$ & .032 & $-.119^{* *}$ & $-.215^{* *}$ & $-.138^{* *}$ & $-.253^{* *}$ & $-.297^{* *}$ & $-.155^{* *}$ & .021 \\
\hline 61. B6w3 & $-.242^{* *}$ & $-.259^{* *}$ & $-.312^{* *}$ & -.040 & $-.195^{* *}$ & $-.281^{* *}$ & $-.289^{* *}$ & $-.284^{* *}$ & $-.404^{* *}$ & $-.279^{* *}$ & $-.136^{* *}$ \\
\hline 62. A1w4 & $.156^{* *}$ & $.082^{*}$ & $.151^{* *}$ & .010 & $.189^{* *}$ & $.143^{* *}$ & $.131^{* *}$ & $.148^{* *}$ & $.151^{* * *}$ & $.121^{* *}$ & .068 \\
\hline 63. $\mathrm{A} 2 \mathrm{w} 4$ & $.225^{* *}$ & $.175^{* *}$ & $.250^{* *}$ & .037 & $.239^{* *}$ & $.263^{* *}$ & $.224^{* *}$ & $.276^{* *}$ & $.268^{* *}$ & $.248^{* *}$ & $.110^{* *}$ \\
\hline 64. $\mathrm{A} 3 \mathrm{w} 4$ & $.219^{* *}$ & $.166^{* *}$ & $.224^{* *}$ & .052 & $.165^{* *}$ & $.205^{* *}$ & $.232^{* *}$ & $.251^{* *}$ & $.252^{* *}$ & $.298^{* *}$ & .035 \\
\hline 65. A4w4 & $.208^{* *}$ & $.140^{* *}$ & $.185^{* *}$ & $.089^{*}$ & $.137^{* *}$ & $.212^{* *}$ & $.223^{* *}$ & $.276^{* *}$ & $.221^{* *}$ & $.306^{* *}$ & $.154^{* *}$ \\
\hline 66. A5w4 & $.245^{* *}$ & $.237^{* *}$ & $.222^{* *}$ & $.130^{* *}$ & $.222^{* *}$ & $.275^{* *}$ & $.259^{* *}$ & $.282^{* *}$ & $.283^{* *}$ & $.272^{* *}$ & $.124^{* *}$ \\
\hline 67. A6w4 & $.244^{* *}$ & $.313^{* *}$ & $.264^{* *}$ & .070 & $.195^{* *}$ & $.288^{* *}$ & $.296^{* *}$ & $.280^{* *}$ & $.313^{\text {** }}$ & $.252^{* *}$ & .068 \\
\hline 68. A7w4 & $.224^{* *}$ & $.250^{* *}$ & $.297^{* *}$ & $.083^{*}$ & $.200^{* *}$ & $.313^{* *}$ & $.263^{* *}$ & $.324^{* *}$ & $.388^{* *}$ & $.278^{* *}$ & $.141^{* *}$ \\
\hline 69. A8w4 & .062 & .054 & .044 & $.142^{* *}$ & $.129^{* *}$ & $.078^{*}$ & .028 & $.131^{* *}$ & .055 & .066 & .051 \\
\hline 70. A9w4 & $.117^{* *}$ & $.305^{* *}$ & $.199^{* *}$ & $.083^{*}$ & $.140^{* *}$ & $.194^{* *}$ & $.165^{* *}$ & $.212^{* *}$ & $.219^{* *}$ & $.211^{* *}$ & $.082^{*}$ \\
\hline 71. A10w4 & $.304^{* * *}$ & $.210^{* * *}$ & $.199^{* * *}$ & $.149^{* * *}$ & $.210^{* * *}$ & $.277^{* *}$ & $.317^{* *}$ & $.272^{* *}$ & $.233^{* *}$ & $.277^{* *}$ & $.187^{* *}$ \\
\hline 72. A11w4 & $.314^{* *}$ & $.216^{* *}$ & $.200^{* *}$ & $.128^{* *}$ & $.278^{* *}$ & $.236^{* *}$ & $.261^{* *}$ & $.256^{* *}$ & $.165^{* *}$ & $.189^{* *}$ & $.147^{* *}$ \\
\hline 73. A12w4 & $.198^{* *}$ & $.352^{* *}$ & $.302^{* *}$ & $.114^{* *}$ & $.203^{* *}$ & $.224^{* *}$ & $.241^{* *}$ & $.268^{* *}$ & $.251^{* *}$ & $.220^{* *}$ & $.137^{* *}$ \\
\hline 74. A13w4 & $.146^{* *}$ & $.181^{* *}$ & $.433^{* *}$ & $.095^{*}$ & $.156^{* *}$ & $.203^{* *}$ & $.235^{* *}$ & $.282^{* *}$ & $.315^{* *}$ & $.343^{* *}$ & .074 \\
\hline 75. A14w4 & $.123^{* *}$ & $.082^{*}$ & $.153^{* *}$ & $.406^{* *}$ & $.154^{* *}$ & $.193^{* *}$ & $.137^{* *}$ & $.151^{* *}$ & $.155^{* *}$ & $.098^{*}$ & $.229^{* *}$ \\
\hline 76. A15w4 & $.177^{* *}$ & $.106^{* *}$ & $.104^{* *}$ & $.080^{*}$ & $.441^{* *}$ & $.176^{* *}$ & $.258^{* *}$ & $.162^{* *}$ & $.136^{* *}$ & $.116^{* *}$ & $.118^{* *}$ \\
\hline 77. A16w4 & $.241^{* *}$ & $.181^{* *}$ & $.291^{* *}$ & $.155^{* *}$ & $.171^{* *}$ & $.386^{* *}$ & $.260^{* *}$ & $.355^{* *}$ & $.365^{* *}$ & $.319^{* *}$ & $.222^{* *}$ \\
\hline
\end{tabular}




\begin{tabular}{|c|c|c|c|c|c|c|c|c|c|c|c|c|}
\hline & & 45. & 46. & 47. & 48. & 49. & 50. & 51. & 52. & 53. & 54. & 55. \\
\hline 78. & $\mathrm{~A} 17 \mathrm{w} 4$ & $.266^{* *}$ & $.226^{* * *}$ & $.290^{* *}$ & $.171^{* *}$ & $.240^{* * *}$ & $.299^{* *}$ & $.424^{* *}$ & $.340^{* *}$ & $.319^{* *}$ & $.310^{* * *}$ & $.229^{* * *}$ \\
\hline 79. & A18w4 & $.269^{* *}$ & $.185^{* *}$ & $.270^{* *}$ & $.213^{* *}$ & $.237^{* *}$ & $.250^{* *}$ & $.272^{* *}$ & $.428^{* *}$ & $.256^{* *}$ & $.264^{* *}$ & $.164^{* *}$ \\
\hline 80. & $\mathrm{~A} 19 \mathrm{w} 4$ & $.228^{* *}$ & $.285^{* *}$ & $.359^{* *}$ & $.122^{* *}$ & $.199^{* *}$ & $.324^{* *}$ & $.281^{* *}$ & $.332^{* *}$ & $.494^{* *}$ & $.307^{* *}$ & $.128^{* *}$ \\
\hline 81. & A20w4 & $.162^{* *}$ & $.128^{* * *}$ & $.277^{* *}$ & $.108^{* *}$ & $.181^{* *}$ & $.229^{* *}$ & $.261^{* *}$ & $.261^{* *}$ & $.281^{* *}$ & $.355^{* *}$ & $.079^{*}$ \\
\hline & A21w4 & $.147^{* *}$ & .016 & $.131^{* *}$ & $.172^{* *}$ & $.142^{* *}$ & $.178^{* *}$ & $.232^{* *}$ & $.195^{* *}$ & $.147^{* *}$ & $.179^{* *}$ & $.382^{* *}$ \\
\hline 83. & B1w4 & $-.116^{* *}$ & $-.140^{* *}$ & $-.205^{* *}$ & .024 & $-.108^{* *}$ & $-.111^{* *}$ & $-.157^{* *}$ & $-.189^{* *}$ & $-.159^{* *}$ & $-.176^{* *}$ & -.049 \\
\hline 84. & B2w4 & -.026 & $-.135^{* *}$ & $-.123^{* *}$ & .065 & -.005 & -.076 & -.059 & $-.095^{*}$ & $-.099^{*}$ & $-.127^{* *}$ & -.013 \\
\hline 85. & B3w4 & $-.140^{* *}$ & $-.184^{* *}$ & $-.164^{* *}$ & -.061 & $-.095^{*}$ & $-.159^{* *}$ & $-.098^{*}$ & $-.163^{* *}$ & $-.174^{* * *}$ & $-.144^{* *}$ & -.025 \\
\hline 86. & B4w4 & $-.172^{* *}$ & $-.203^{* *}$ & $-.311^{* *}$ & -.041 & $-.119^{* *}$ & $-.200^{* *}$ & $-.188^{* *}$ & $-.306^{* *}$ & $-.299^{* *}$ & $-.312^{* *}$ & -.035 \\
\hline 87. & B5w4 & $-.113^{* *}$ & $-.153^{* *}$ & $-.171^{* *}$ & -.004 & -.074 & $-.140^{* *}$ & $-.150^{* *}$ & $-.245^{* *}$ & $-.217^{* *}$ & $-.142^{* *}$ & -.053 \\
\hline 88. & B6w4 & $-.133^{* *}$ & $-.190^{* *}$ & $-.278^{* *}$ & -.037 & $-.132^{* *}$ & $-.176^{* *}$ & $-.190^{* *}$ & $-.284^{* *}$ & $-.300^{* * *}$ & $-.215^{* *}$ & $-.088^{*}$ \\
\hline 89. & Instability & .009 & .026 & $-.098^{* *}$ & .022 & .029 & .005 & .038 & .006 & -.018 & -.052 & .024 \\
\hline & & 56. & 57. & 58. & 59. & 60. & 61. & 62. & 63. & 64. & 65. & 66. \\
\hline
\end{tabular}

56. B1w3 1

57. B2w3 .215** 1

58. B3w3 .179** $.240^{* *} \quad 1$

59. B4w3 .239** $.316^{* *} \quad .302^{* *} \quad 1$

60. B5w3 $\quad .213^{* *} \quad .183^{* *} \quad .286^{* *} \quad .319^{* *} \quad 1$

61. B6w3 $\quad .243^{* *} \quad .197^{* *} \quad .311^{* *} \quad .440^{* *} \quad .453^{* *} \quad 1$

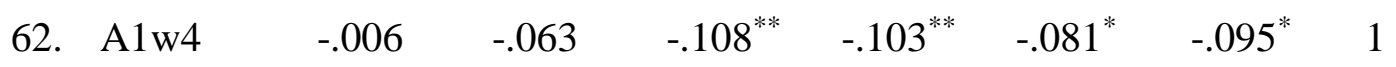

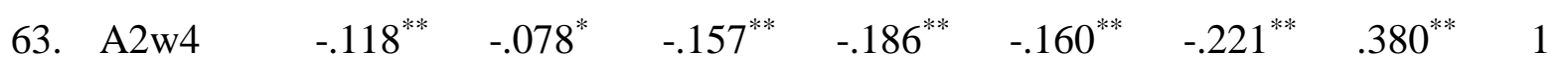

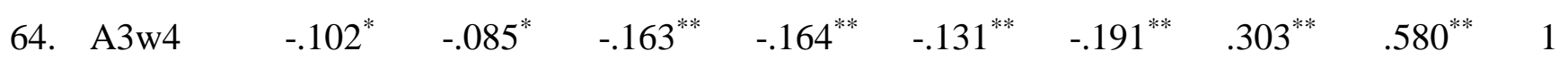

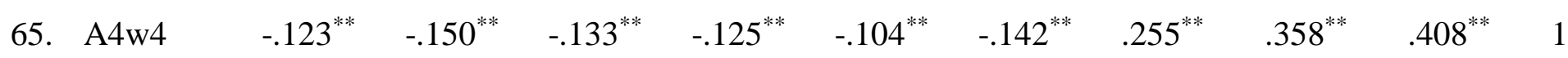




\begin{tabular}{|c|c|c|c|c|c|c|c|c|c|c|c|c|}
\hline & & 56. & 57. & 58. & 59. & 60. & 61. & 62. & 63. & 64. & 65. & 66. \\
\hline 66. & $\mathrm{~A} 5 \mathrm{w} 4$ & $-.148^{* * *}$ & $-.196^{* *}$ & $-.169^{* *}$ & $-.201^{* *}$ & $-.203^{* *}$ & $-.204^{* *}$ & $.226^{* *}$ & $.394^{* *}$ & $.353^{* *}$ & $.312^{* * *}$ & 1 \\
\hline & A6w4 & $-.165^{* *}$ & $-.188^{* *}$ & $-.205^{* *}$ & $-.228^{* *}$ & $-.181^{* *}$ & $-.233^{* *}$ & $.233^{* *}$ & $.395^{* *}$ & $.336^{* *}$ & $.256^{* *}$ & $.679^{* *}$ \\
\hline 68. & $\mathrm{~A} 7 \mathrm{w} 4$ & $-.168^{* *}$ & $-.212^{* *}$ & $-.223^{* *}$ & $-.217^{* *}$ & $-.256^{* *}$ & $-.275^{* *}$ & $.254^{* *}$ & $.396^{* *}$ & $.355^{* *}$ & $.313^{* *}$ & $.438^{* *}$ \\
\hline 69. & A8w4 & .003 & .016 & .031 & -.005 & -.023 & -.033 & -.031 & $.097^{*}$ & .053 & $.110^{* * *}$ & $.078^{*}$ \\
\hline 70 . & $\mathrm{A} 9 \mathrm{w} 4$ & $-.152^{* * *}$ & $-.203^{* *}$ & $-.145^{* *}$ & $-.161^{* *}$ & $-.114^{* *}$ & $-.106^{* *}$ & $.100^{* *}$ & $.266^{* *}$ & $.261^{* * *}$ & $.203^{* *}$ & $.309^{* *}$ \\
\hline 71. & A10w4 & -.051 & -.069 & $-.152^{* *}$ & $-.150^{* *}$ & $-.132^{* * *}$ & $-.162^{* *}$ & $.281^{* *}$ & $.406^{* *}$ & $.407^{* *}$ & $.349^{* * *}$ & $.384^{* *}$ \\
\hline 72. & A11w4 & -.018 & $-.079^{*}$ & -.073 & -.060 & -.051 & $-.088^{*}$ & $.171^{* *}$ & $.267^{* * *}$ & $.252^{* * *}$ & $.247^{* * *}$ & $.319^{* *}$ \\
\hline 73. & $\mathrm{~A} 12 \mathrm{w} 4$ & $-.122^{* *}$ & $-.203^{* *}$ & $-.148^{* *}$ & $-.212^{* *}$ & $-.151^{* * *}$ & $-.124^{* * *}$ & $.111^{* *}$ & $.267^{* *}$ & $.242^{* *}$ & $.265^{* *}$ & $.414^{* *}$ \\
\hline 74. & $\mathrm{~A} 13 \mathrm{w} 4$ & $-.121^{* *}$ & $-.184^{* *}$ & $-.201^{* *}$ & $-.161^{* *}$ & $-.173^{* *}$ & $-.184^{* *}$ & $.140^{* *}$ & $.250^{* * *}$ & $.257^{* * *}$ & $.357^{* * *}$ & $.315^{* *}$ \\
\hline 75. & A14w4 & .013 & -.054 & .004 & $-.093^{*}$ & -.002 & -.071 & $.084^{*}$ & $.174^{* *}$ & $.110^{* *}$ & $.146^{* *}$ & $.175^{* *}$ \\
\hline 76. & A15w4 & -.046 & -.061 & $-.119^{* *}$ & $-.111^{* *}$ & -.048 & -.060 & $.256^{* *}$ & $.302^{* *}$ & $.210^{* * *}$ & $.177^{* * *}$ & $.258^{* *}$ \\
\hline 77. & A16w4 & $-.086^{*}$ & $-.119^{* *}$ & $-.181^{* *}$ & $-.175^{* *}$ & $-.208^{* *}$ & $-.203^{* *}$ & $.186^{* *}$ & $.335^{* *}$ & $.287^{* *}$ & $.372^{* * *}$ & $.350^{* *}$ \\
\hline 78. & $\mathrm{~A} 17 \mathrm{w} 4$ & $-.153^{* *}$ & $-.138^{* *}$ & $-.167^{* *}$ & $-.177^{* *}$ & $-.166^{* *}$ & $-.229^{* *}$ & $.211^{* *}$ & $.348^{* * *}$ & $.294^{* * *}$ & $.350^{* * *}$ & $.359^{* * *}$ \\
\hline 79. & A18w4 & $-.090^{*}$ & $-.114^{* *}$ & $-.127^{* *}$ & $-.099^{*}$ & $-.166^{* *}$ & $-.194^{* *}$ & $.160^{* *}$ & $.302^{* *}$ & $.263^{* *}$ & $.339^{* *}$ & $.345^{* *}$ \\
\hline 80. & A19w4 & $-.161^{* *}$ & $-.170^{* *}$ & $-.220^{* *}$ & $-.212^{* *}$ & $-.230^{* *}$ & $-.282^{* *}$ & $.181^{* *}$ & $.366^{* *}$ & $.306^{* *}$ & $.402^{* *}$ & $.403^{* *}$ \\
\hline 81. & A20w4 & $-.162^{* *}$ & $-.087^{*}$ & $-.159^{* *}$ & $-.160^{* *}$ & $-.099^{*}$ & $-.194^{* *}$ & $.111^{* *}$ & $.301^{* *}$ & $.320^{* * *}$ & $.341^{* * *}$ & $.334^{* *}$ \\
\hline & $\mathrm{A} 21 \mathrm{w} 4$ & -.044 & -.070 & -.044 & -.046 & -.016 & $-.103^{* *}$ & $.113^{* *}$ & $.164^{* *}$ & $.082^{*}$ & $.270^{* *}$ & $.196^{* *}$ \\
\hline & B1w4 & $.249^{* *}$ & $.120^{* *}$ & $.179^{* *}$ & $.244^{* *}$ & $.153^{* *}$ & $.219^{* * *}$ & $-.120^{* *}$ & $-.196^{* *}$ & $-.234^{* *}$ & $-.206^{* *}$ & $-.145^{* *}$ \\
\hline 84. & $\mathrm{~B} 2 \mathrm{w} 4$ & $.083^{*}$ & $.271^{* *}$ & $.201^{* * *}$ & $.087^{*}$ & $.085^{*}$ & $.102^{*}$ & $-.090^{*}$ & $-.175^{* *}$ & $-.109^{* *}$ & $-.121^{* * *}$ & $-.110^{* *}$ \\
\hline & B3w4 & $.137^{* *}$ & $.162^{* *}$ & $.164^{* * *}$ & $.153^{* *}$ & $.187^{* *}$ & $.139^{* *}$ & $-.162^{* *}$ & $-.305^{* *}$ & $-.279^{* *}$ & $-.182^{* *}$ & $-.274^{* *}$ \\
\hline & B4w4 & $.241^{* *}$ & $.187^{* *}$ & $.259^{* * *}$ & $.328^{* *}$ & $.209^{* *}$ & $.286^{* *}$ & $-.141^{* * *}$ & $-.289^{* *}$ & $-.303^{* *}$ & $-.237^{* *}$ & $-.307^{* *}$ \\
\hline & B5w4 & $.160^{* *}$ & $.141^{* *}$ & $.206^{* *}$ & $.191^{* *}$ & $.321^{* *}$ & $.304^{* *}$ & -.054 & $-.198^{* *}$ & $-.204^{* *}$ & $-.196^{* *}$ & $-.182^{* *}$ \\
\hline 88. & B6w4 & $.168^{* *}$ & $.202^{* *}$ & $.200^{* *}$ & $.260^{* *}$ & $.242^{* *}$ & $.360^{* *}$ & -.063 & $-.201^{* *}$ & $-.265^{* *}$ & $-.230^{* *}$ & $-.293^{* *}$ \\
\hline 89. & Instability & .045 & -.011 & -.045 & $-.081^{*}$ & .048 & .007 & $.093^{*}$ & .043 & .020 & .009 & .043 \\
\hline
\end{tabular}




\begin{tabular}{|c|c|c|c|c|c|c|c|c|c|c|c|c|}
\hline & & 67. & 68. & 69. & 70. & 71. & 72. & 73. & 74. & 75. & 76. & 77. \\
\hline 67. & A6w4 & 1 & & & & & & & & & & \\
\hline 68. & A7w4 & $.446^{* * *}$ & 1 & & & & & & & & & \\
\hline 69. & A8w4 & .038 & $.101^{* *}$ & 1 & & & & & & & & \\
\hline 70. & A9w4 & $.365^{* *}$ & $.350^{* *}$ & $.097^{*}$ & 1 & & & & & & & \\
\hline 71. & A10w4 & $.336^{* * *}$ & $.362^{* *}$ & $.121^{* *}$ & $.287^{* *}$ & 1 & & & & & & \\
\hline 72. & A11w4 & $.298^{* *}$ & $.248^{* *}$ & $.228^{* * *}$ & $.272^{* *}$ & $.469^{* *}$ & 1 & & & & & \\
\hline 73. & $\mathrm{~A} 12 \mathrm{w} 4$ & $.420^{* * *}$ & $.416^{* *}$ & $.101^{* *}$ & $.478^{* *}$ & $.312^{* *}$ & $.329^{* * *}$ & 1 & & & & \\
\hline 74. & $\mathrm{~A} 13 \mathrm{w} 4$ & $.352^{* * *}$ & $.373^{* *}$ & .039 & $.301^{* *}$ & $.283^{* *}$ & $.269^{* *}$ & $.400^{* *}$ & 1 & & & \\
\hline 75. & A14w4 & $.111^{* *}$ & $.133^{* *}$ & $.193^{* *}$ & $.177^{* *}$ & $.232^{* *}$ & $.235^{* *}$ & $.224^{* *}$ & $.119^{* *}$ & 1 & & \\
\hline 76. & A15w4 & $.241^{* * *}$ & $.195^{* *}$ & $.077^{*}$ & $.156^{* *}$ & $.329^{* * *}$ & $.294^{* *}$ & $.277^{* *}$ & $.206^{* *}$ & $.163^{* *}$ & 1 & \\
\hline 77. & A16w4 & $.333^{* * *}$ & $.442^{* *}$ & .049 & $.283^{* *}$ & $.369^{* * *}$ & $.262^{* * *}$ & $.349^{* * *}$ & $.401^{* *}$ & $.234^{* *}$ & $.214^{* * *}$ & \\
\hline 78. & $\mathrm{~A} 17 \mathrm{w} 4$ & $.402^{* *}$ & $.382^{* *}$ & $.119^{* * *}$ & $.331^{* *}$ & $.396^{* *}$ & $.307^{* * *}$ & $.352^{* * *}$ & $.408^{* *}$ & $.253^{* *}$ & $.270^{* *}$ & $.485^{* *}$ \\
\hline 79. & $\mathrm{~A} 18 \mathrm{w} 4$ & $.306^{* * *}$ & $.372^{* *}$ & $.097^{*}$ & $.243^{* * *}$ & $.303^{* *}$ & $.299^{* *}$ & $.311^{* *}$ & $.335^{* *}$ & $.308^{* *}$ & $.182^{* *}$ & $.497^{* *}$ \\
\hline 80. & A19w4 & $.410^{* * *}$ & $.464^{* *}$ & $.098^{* * *}$ & $.363^{* *}$ & $.388^{* * *}$ & $.279^{* * *}$ & $.450^{* * *}$ & $.484^{* *}$ & $.248^{* *}$ & $.254^{* *}$ & $.587^{* *}$ \\
\hline 81. & A20w4 & $.338^{* *}$ & $.374^{* *}$ & .044 & $.302^{* *}$ & $.289^{* * *}$ & $.267^{* *}$ & $.292^{* *}$ & $.490^{* *}$ & $.168^{* *}$ & $.187^{* *}$ & $.423^{* *}$ \\
\hline 82. & $\mathrm{~A} 21 \mathrm{w} 4$ & $.110^{* * *}$ & $.202^{* *}$ & $.152^{* * *}$ & $.107^{* * *}$ & $.280^{* *}$ & $.288^{* *}$ & $.186^{* *}$ & $.189^{* *}$ & $.319^{* *}$ & $.215^{* *}$ & $.291^{* *}$ \\
\hline 83. & B1w4 & $-.207^{* *}$ & $-.196^{* *}$ & $-.083^{*}$ & $-.144^{* *}$ & $-.162^{* *}$ & $-.122^{* *}$ & $-.201^{* *}$ & $-.227^{* *}$ & -.045 & $-.119^{* *}$ & $-.164^{* *}$ \\
\hline 84. & B2w4 & $-.161^{* *}$ & $-.152^{* *}$ & -.021 & $-.195^{* *}$ & -.069 & -.064 & $-.138^{* *}$ & $-.124^{* *}$ & -.070 & .015 & $-.105^{* *}$ \\
\hline 85. & B3w4 & $-.279^{* *}$ & $-.258^{* *}$ & -.029 & $-.221^{* *}$ & $-.176^{* *}$ & $-.135^{* *}$ & $-.231^{* * *}$ & $-.205^{* *}$ & -.064 & $-.137^{* *}$ & $-.202^{* *}$ \\
\hline 86. & B4w4 & $-.350^{* *}$ & $-.368^{* *}$ & -.070 & $-.340^{* *}$ & $-.288^{* *}$ & $-.211^{* *}$ & $-.374^{* *}$ & $-.340^{* *}$ & $-.133^{* *}$ & $-.118^{* *}$ & $-.369^{* *}$ \\
\hline 87. & B5w4 & $-.208^{* *}$ & $-.300^{* *}$ & -.016 & $-.182^{* *}$ & $-.179^{* *}$ & $-.141^{* *}$ & $-.257^{* * *}$ & $-.292^{* *}$ & $-.076^{*}$ & $-.112^{* *}$ & $-.305^{* *}$ \\
\hline 88. & B6w4 & $-.309^{* *}$ & $-.361^{* *}$ & -.050 & $-.192^{* *}$ & $-.218^{* *}$ & $-.155^{* *}$ & $-.298^{* * *}$ & $-.379^{* *}$ & $-.091^{*}$ & $-.146^{* *}$ & $-.314^{* * *}$ \\
\hline 89. & Instability & $.093^{*}$ & .036 & .044 & .050 & $.135^{* *}$ & .039 & .044 & -.017 & .026 & $.113^{* *}$ & .060 \\
\hline
\end{tabular}




\begin{tabular}{|c|c|c|c|c|c|c|c|c|c|c|c|c|c|}
\hline & & 78. & 79. & 80. & 81. & 82. & 83. & 84. & 85. & 86. & 87. & 88. & 89. \\
\hline 78. & $\mathrm{~A} 17 \mathrm{w} 4$ & 1 & & & & & & & & & & & \\
\hline 79. & A18w4 & $.477^{* *}$ & 1 & & & & & & & & & & \\
\hline 80. & A19w4 & $.514^{* *}$ & $.446^{* *}$ & 1 & & & & & & & & & \\
\hline 81. & A20w4 & $.416^{* *}$ & $.337^{* *}$ & $.466^{* *}$ & 1 & & & & & & & & \\
\hline 82. & A21w4 & $.316^{* *}$ & $.282^{* *}$ & $.237^{* *}$ & $.197^{* *}$ & 1 & & & & & & & \\
\hline 83. & B1w4 & $-.205^{* *}$ & $-.180^{* *}$ & $-.286^{* *}$ & $-.242^{* *}$ & -.033 & 1 & & & & & & \\
\hline 84. & $\mathrm{~B} 2 \mathrm{w} 4$ & $-.094^{*}$ & -.029 & $-.111^{* *}$ & $-.100^{* *}$ & -.027 & $.123^{* *}$ & 1 & & & & & \\
\hline 85. & B3w4 & $-.216^{* *}$ & $-.199^{* *}$ & $-.251^{* *}$ & $-.175^{* *}$ & -.073 & $.147^{* *}$ & $.255^{* *}$ & 1 & & & & \\
\hline 86. & B4w4 & $-.280^{* *}$ & $-.267^{* *}$ & $-.389^{* *}$ & $-.279^{* *}$ & $-.129^{* *}$ & $.244^{* *}$ & $.300^{* *}$ & $.299^{* *}$ & 1 & & & \\
\hline 87. & B5w4 & $-.246^{* *}$ & $-.244^{* *}$ & $-.336^{* *}$ & $-.190^{* *}$ & -.072 & $.307^{* *}$ & $.165^{* *}$ & $.187^{* *}$ & $.331^{* *}$ & 1 & & \\
\hline 88. & B6w4 & $-.298^{* *}$ & $-.303^{* *}$ & $-.453^{* *}$ & $-.268^{* *}$ & $-.116^{* *}$ & $.304^{* *}$ & $.177^{* *}$ & $.278^{* *}$ & $.434^{* *}$ & $.531^{* *}$ & 1 & \\
\hline 89. & Instability & $.102^{* *}$ & .046 & .023 & -.022 & -.032 & .033 & -.012 & -.040 & -.060 & -.005 & .000 & 1 \\
\hline
\end{tabular}




\section{Appendix K: Item Probabilities from LCA Models}

Table K1

Wave 1 Probability of Problem Behaviors for Latent Classes 1 and 2

\begin{tabular}{|c|c|c|c|c|}
\hline & Item text & Subscale & $\begin{array}{c}\text { Class } 1 \\
\text { (Dysregulated) }\end{array}$ & $\begin{array}{c}\text { Class } 2 \\
\text { (Regulated) }\end{array}$ \\
\hline A1 & acts too young for age & Attention & 0.395 & 0.154 \\
\hline $\mathrm{A} 2$ & $\begin{array}{c}\text { can't concentrate, can't pay } \\
\text { attention for long }\end{array}$ & Attention & 0.753 & 0.403 \\
\hline A3 & can't sit still, restless, hyperactive & Attention & 0.916 & 0.526 \\
\hline A4 & demands a lot of attention & Aggressive & 0.941 & 0.564 \\
\hline A5 & destroys his/her own things & Aggressive & 0.620 & 0.124 \\
\hline A6 & $\begin{array}{l}\text { destroys things belonging to } \\
\text { his/her family or others }\end{array}$ & Aggressive & 0.593 & 0.131 \\
\hline A7 & disobedient at home & Aggressive & 0.840 & 0.325 \\
\hline A8 & $\begin{array}{l}\text { fears certain animals, situations, or } \\
\text { places, other than school }\end{array}$ & Anxious/Depressed & 0.564 & 0.291 \\
\hline A9 & gets in may fights & Aggressive & 0.362 & 0.020 \\
\hline A10 & nervous, high-strung, or tense & Anxious/Depressed & 0.437 & 0.051 \\
\hline A11 & too fearful or anxious & Anxious/Depressed & 0.413 & 0.076 \\
\hline A12 & physically attacks people & Aggressive & 0.234 & 0.057 \\
\hline A13 & screams a lot & Aggressive & 0.688 & 0.162 \\
\hline A14 & $\begin{array}{c}\text { self-conscious or easily } \\
\text { embarrassed }\end{array}$ & Anxious/Depressed & 0.591 & 0.333 \\
\hline A15 & stares blankly & Attention & 0.266 & 0.020 \\
\hline A16 & stubborn, sullen, or irritable & Aggressive & 0.940 & 0.474 \\
\hline A17 & $\begin{array}{l}\text { sudden changes in mood or } \\
\text { feelings }\end{array}$ & Aggressive & 0.761 & 0.181 \\
\hline A18 & sulks a lot & Aggressive & 0.765 & 0.275 \\
\hline A19 & temper tantrums or hot temper & Aggressive & 0.894 & 0.417 \\
\hline A 20 & unusually loud & Aggressive & 0.732 & 0.291 \\
\hline $\mathrm{A} 21$ & worries & Anxious/Depressed & 0.337 & 0.121 \\
\hline B1 & inappropriate tone of voice & $\begin{array}{l}\text { Self-control } \\
\text { problems }\end{array}$ & 0.301 & 0.671 \\
\hline $\mathrm{B} 2$ & inappropriate response when hit & $\begin{array}{l}\text { Self-control } \\
\text { problems }\end{array}$ & 0.284 & 0.480 \\
\hline B3 & gets in troubling situations & $\begin{array}{l}\text { Self-control } \\
\text { problems }\end{array}$ & 0.158 & 0.394 \\
\hline B4 & temper problems with kids & $\begin{array}{l}\text { Self-control } \\
\text { problems }\end{array}$ & 0.098 & 0.311 \\
\hline B5 & doesn't calmly end disagreements & $\begin{array}{l}\text { Self-control } \\
\text { problems }\end{array}$ & 0.133 & 0.353 \\
\hline
\end{tabular}




\begin{tabular}{|c|c|c|c|c|}
\hline & Item text & Subscale & $\begin{array}{c}\text { Class } 1 \\
\text { (Dysregulated) }\end{array}$ & $\begin{array}{c}\text { Class } 2 \\
\text { (Regulated) }\end{array}$ \\
\hline B6 & temper problems with caregiver & $\begin{array}{l}\text { Self-control } \\
\text { problems }\end{array}$ & 0.116 & 0.444 \\
\hline
\end{tabular}

Table K2

Wave 3 Probability of Problem Behaviors for Latent Classes 1 and 2

\begin{tabular}{|c|c|c|c|c|}
\hline & Item text & Subscale & $\begin{array}{c}\text { Class } 1 \\
\text { (Dysregulated) }\end{array}$ & $\begin{array}{c}\text { Class } 2 \\
\text { (Regulated) }\end{array}$ \\
\hline A1 & acts too young for age & Attention & 0.553 & 0.294 \\
\hline $\mathrm{A} 2$ & $\begin{array}{l}\text { can't concentrate, can't pay } \\
\text { attention for long }\end{array}$ & Attention & 0.831 & 0.445 \\
\hline A3 & $\begin{array}{c}\text { can't sit still, restless, } \\
\text { hyperactive }\end{array}$ & Attention & 0.904 & 0.520 \\
\hline A4 & demands a lot of attention & Aggressive & 0.874 & 0.530 \\
\hline A5 & destroys his/her own things & Aggressive & 0.664 & 0.110 \\
\hline A6 & $\begin{array}{l}\text { destroys things belonging to } \\
\text { his/her family or others }\end{array}$ & Aggressive & 0.555 & 0.096 \\
\hline A7 & disobedient at home & Aggressive & 0.889 & 0.283 \\
\hline A8 & $\begin{array}{l}\text { fears certain animals, situations, } \\
\text { or places, other than school }\end{array}$ & Anxious & 0.548 & 0.396 \\
\hline A9 & gets in may fights & Aggressive & 0.257 & 0.020 \\
\hline A10 & nervous, high-strung, or tense & Anxious & 0.537 & 0.073 \\
\hline A11 & too fearful or anxious & Anxious & 0.410 & 0.041 \\
\hline A12 & physically attacks people & Aggressive & 0.283 & 0.005 \\
\hline A13 & screams a lot & Aggressive & 0.611 & 0.105 \\
\hline A14 & $\begin{array}{l}\text { self-conscious or easily } \\
\text { embarrassed }\end{array}$ & Anxious & 0.623 & 0.322 \\
\hline A15 & stares blankly & Attention & 0.262 & 0.011 \\
\hline A16 & stubborn, sullen, or irritable & Aggressive & 0.932 & 0.344 \\
\hline A17 & $\begin{array}{l}\text { sudden changes in mood or } \\
\text { feelings }\end{array}$ & Aggressive & 0.743 & 0.231 \\
\hline A18 & sulks a lot & Aggressive & 0.759 & 0.219 \\
\hline A19 & temper tantrums or hot temper & Aggressive & 0.914 & 0.312 \\
\hline $\mathrm{A} 20$ & unusually loud & Aggressive & 0.718 & 0.268 \\
\hline $\mathrm{A} 21$ & worries & Anxious & 0.462 & 0.130 \\
\hline B1 & inappropriate tone of voice & $\begin{array}{l}\text { Self-control } \\
\text { problems }\end{array}$ & 0.358 & 0.614 \\
\hline B2 & inappropriate response when hit & $\begin{array}{l}\text { Self-control } \\
\text { problems }\end{array}$ & 0.288 & 0.445 \\
\hline B3 & gets in troubling situations & $\begin{array}{l}\text { Self-control } \\
\text { problems }\end{array}$ & 0.131 & 0.389 \\
\hline
\end{tabular}




\begin{tabular}{|c|c|c|c|c|}
\hline & Item text & Subscale & $\begin{array}{c}\text { Class } 1 \\
\text { (Dysregulated) }\end{array}$ & $\begin{array}{c}\text { Class } 2 \\
\text { (Regulated) }\end{array}$ \\
\hline B4 & temper problems with kids & $\begin{array}{c}\text { Self-control } \\
\text { problems }\end{array}$ & 0.165 & 0.434 \\
\hline B5 & $\begin{array}{l}\text { doesn't calmly end } \\
\text { disagreements }\end{array}$ & $\begin{array}{l}\text { Self-control } \\
\text { problems }\end{array}$ & 0.131 & 0.368 \\
\hline B6 & temper problems with caregiver & $\begin{array}{c}\text { Self-control } \\
\text { problems }\end{array}$ & 0.202 & 0.521 \\
\hline
\end{tabular}

Table K3

Wave 4 Probability of Problem Behaviors for Latent Classes 1 and 2

\begin{tabular}{|c|c|c|c|c|}
\hline & Item text & Subscale & $\begin{array}{c}\text { Class } 1 \\
\text { (Dysregulated) } \\
\end{array}$ & $\begin{array}{c}\text { Class } 2 \\
\text { (Regulated) } \\
\end{array}$ \\
\hline A1 & acts too young for age & Attention & 0.636 & 0.319 \\
\hline $\mathrm{A} 2$ & $\begin{array}{l}\text { can't concentrate, can't pay } \\
\text { attention for long }\end{array}$ & Attention & 0.866 & 0.473 \\
\hline A3 & $\begin{array}{c}\text { can't sit still, restless, } \\
\text { hyperactive }\end{array}$ & Attention & 0.887 & 0.543 \\
\hline A4 & demands a lot of attention & Aggressive & 0.858 & 0.450 \\
\hline A5 & destroys his/her own things & Aggressive & 0.643 & 0.057 \\
\hline A6 & $\begin{array}{l}\text { destroys things belonging to } \\
\text { his/her family or others }\end{array}$ & Aggressive & 0.668 & 0.125 \\
\hline A7 & disobedient at home & Aggressive & 0.831 & 0.270 \\
\hline A8 & $\begin{array}{c}\text { fears certain animals, situations, } \\
\text { or places, other than school }\end{array}$ & Anxious & 0.354 & 0.320 \\
\hline A9 & gets in may fights & Aggressive & 0.322 & 0.009 \\
\hline A10 & nervous, high-strung, or tense & Anxious & 0.548 & 0.130 \\
\hline A11 & too fearful or anxious & Anxious & 0.498 & 0.094 \\
\hline A12 & physically attacks people & Aggressive & 0.349 & 0.001 \\
\hline A13 & screams a lot & Aggressive & 0.676 & 0.148 \\
\hline A14 & $\begin{array}{c}\text { self-conscious or easily } \\
\text { embarrassed }\end{array}$ & Anxious & 0.629 & 0.431 \\
\hline A15 & stares blankly & Attention & 0.326 & 0.069 \\
\hline A16 & stubborn, sullen, or irritable & Aggressive & 0.905 & 0.408 \\
\hline A17 & $\begin{array}{l}\text { sudden changes in mood or } \\
\text { feelings }\end{array}$ & Aggressive & 0.717 & 0.244 \\
\hline A18 & sulks a lot & Aggressive & 0.682 & 0.245 \\
\hline A19 & temper tantrums or hot temper & Aggressive & 0.843 & 0.292 \\
\hline A20 & unusually loud & Aggressive & 0.692 & 0.306 \\
\hline A21 & worries & Anxious & 0.528 & 0.165 \\
\hline B1 & inappropriate tone of voice & $\begin{array}{l}\text { Self-control } \\
\text { problems }\end{array}$ & 0.364 & 0.566 \\
\hline
\end{tabular}




\begin{tabular}{|c|c|c|c|c|}
\hline & Item text & Subscale & $\begin{array}{c}\text { Class 1 } \\
\text { (Dysregulated) }\end{array}$ & $\begin{array}{c}\text { Class } 2 \\
\text { (Regulated) }\end{array}$ \\
\hline B2 & inappropriate response when hit & $\begin{array}{l}\text { Self-control } \\
\text { problems }\end{array}$ & 0.207 & 0.326 \\
\hline B3 & gets in troubling situations & $\begin{array}{l}\text { Self-control } \\
\text { problems }\end{array}$ & 0.112 & 0.393 \\
\hline B4 & temper problems with kids & $\begin{array}{l}\text { Self-control } \\
\text { problems }\end{array}$ & 0.067 & 0.462 \\
\hline B5 & $\begin{array}{l}\text { doesn't calmly end } \\
\text { disagreements }\end{array}$ & $\begin{array}{l}\text { Self-control } \\
\text { problems }\end{array}$ & 0.193 & 0.455 \\
\hline B6 & temper problems with caregiver & $\begin{array}{c}\text { Self-control } \\
\text { problems }\end{array}$ & 0.156 & 0.600 \\
\hline
\end{tabular}




\section{References}

Achenbach, T. M. (1991). Manual for the Child Behavior Checklist/4-18 and the 1991 Profile. Burlington, VT: University of Vermont Department of Psychiatry.

Adrian, M., Zeman, J., \& Veits, G. (2011). Methodological implications of the affective revolution: A 35-year review of emotion regulation assessment in children. Journal of Experimental Child Psychology, 110, 171-197.

Alink, L. R. A., Cicchetti, D., Kim, J., \& Rogosch, F. A., (2009). Mediating and moderating processes in the relation between maltreatment and psychopathology: Mother-child relationship quality and emotion regulation. Journal of Abnormal Child Psychology, 37, 831-843.

Althoff, R. (2010). Dysregulated children reconsidered. Journal of the American Academy of Child \& Adolescent Psychiatry, 49, 302-305.

Althoff, R. R., Ayer, L. A., Rettew, D. C., \& Hudziak, J. J. (2010). Assessment of dysregulated children using the Child Behavior Checklist: A receiver operating characteristic curve analysis. Psychological Assessment, 22, 609-617.

Althoff, R. R., Rettew, D. C., Faraone, S. V., Boomsman, D. I., \& Hudziak, J. J. (2006). Latent class analysis shows strong heritability of the Child Behavior Checklist - juvenile bipolar phenotype. Biological Psychiatry, 60, 903-911.

Altschuler, S. J. (1997). A reveille for school social workers: Children in foster care need our help! Social Work in Education, 19, 121-127.

American Psychiatric Association. (2000). Diagnostic and statistical manual of mental disorders ( $4^{\text {th }}$ ed., text rev.). Washington, DC. 
Asparouhov, T. (2005). Sampling weights in latent variable modeling. Structural Equation Modeling, 12, 411-434.

Ayer, L., Althoff, R., Ivanova, M., Rettew, D., Waxler, E., Sulman, J., \& Hudziak, J. (2009). Child behavior checklist juvenile bipolar disorder (CBCL-JBD) and CBCL posttraumatic stress problems (CBCL-PTSP) scales are measures of a single dysregulatory syndrome. The Journal of Child Psychology and Psychiatry, 50, 1291-1300.

Bae, H., Solomon, P. L., \& Gelles, R. J. (2009). Multiple child maltreatment recurrence relative to single recurrence and no recurrence. Children and Youth Services Review, 31, 617-624.

Baker, J. A. (2006). Contributions of Teacher-Child Relationships to Positive School Adjustment during Elementary School. Journal of School Psychology, 44, 211-229.

Bandalos, D.L., \& Finney, S.J. (2010). Factor analysis: Exploratory and confirmatory. In G.R. Hancock \& R.O. Mueller (Eds.), The reviewer's guide to quantitative methods in the social sciences (pp. 125-155). Florence, KY: Routledge Education.

Barnett, D., Manly, J. T., \& Cicchetti, D. (1993). Defining child maltreatment: The interface between policy and research. Child Abuse, Child Development, and Social Policy, 8, 773.

Barrat, V. X., \& Berliner, B. (2013). The invisible achievement gap, part 1: Education outcomes of students in foster care in California's public schools. Retrieved from http://www.stuartfoundation.org/docs/default-document-library/the-invisibleachievement-gap-report.

Bergman, L. R., \& Vargha, A. (2013). Matching method to problem: A developmental science perspective. European Journal of Developmental Psychology, 10, 9-28. 
Blair, C. (2002). School readiness: Integrating cognition and emotion in a neurobiological conceptualization of children's functioning at school entry. American Psychologist, 57, $111-127$.

Blair, C., \& Raver, C. C. (2012). Child development in the context of adversity: Experiential canalization of brain and behavior. American Psychologist, 67, 309-318. doi: 10.1037/a0027493.

Blandon, A., Calkins, S., Keane, S., \& O'Brien, M. (2010). Contributions of child's physiology and maternal behavior to children's trajectories of temperamental reactivity. Developmental Psychology, 46(5), 1089-1102. doi:10.1037/a0020678

Bronfenbrenner, U., \& Ceci, S. J. (1994). Nature-nurture reconceptualized in developmental perspective: A Bio-ecological Model. Psychological Review, 10, 568-586.

Bronfenbrenner, U., \& Morris, P. A. (1998). The ecology of developmental processes. In W. Damon \& R. M. Lerner (Eds.), Handbook of child psychology, Vol. 1: Theoretical models of human development ( $5^{\text {th }}$ ed., pp. 993-1023). New York: Wiley.

Bronfenbrenner, U., \& Morris, P. A. (2006). The bio-ecological model of human development. In W. Damon \& R. M. Lerner (Eds.), Handbook of child psychology, Vol. 1: Theoretical models of human development (6 ${ }^{\text {th }}$ ed., pp. 793-828). New York: Wiley.

Bub, K. L., Buckhalt, J. A., \& El-Sheikh, M. (2011). Children's sleep and cognitive performance: A cross-domain analysis of change over time. Developmental Psychology, 47, 15041514. doi:10.1037/a0025535.

Burchinal, M., Howes, C., Pianta, R., Bryant, D., Early, D., Clifford, R., \& Barbarin, O. (2008). Predicting child outcomes at the end of kindergarten from the quality of pre-kindergarten teacher-child interactions and instruction. Applied Developmental Science, 12, 140-153. 
Caldwell, B., \& Bradley, R. (1984). Home observation for measurement of the environment: Administration manual.

Calkins, S. (1997). Cardiac vagal tone indices of temperamental reactivity and behavioral regulation in young children. Developmental Psychobiology, 21, 125-135.

Calkins, S. D., \& Hill, A. (2007). Caregiver influences on emerging emotion regulation: Biological and environmental transactions in early development. In J. J. Gross (Ed.), Handbook of emotion regulation (pp. 229-248). New York, NY US: Guilford Press.

Casanueva, C., Cross, T. P., Ringeisen, H., \& Christ, S. L. (2011). Prevalence, trajectories, and risk factors for depression among caregivers of young children involved in child maltreatment investigations. Journal of Emotional and Behavioral Disorders, 19, 98-116.

Casanueva, C., Dozier, M., Tueller, S., Dolan, M., Smith, K., Bruce Webb, M., Westbrook, T., \& Jones Harden, B. (2014). Caregiver instability and early life changes among infants reported to the child welfare system. Child Abuse and Neglect, 38, 498-509.

Casanueva, C., Martin, S. L., \& Runyan, D. K. (2009). Repeated reports for child maltreatment among intimate partner violence victims: Findings from the National Survey of Child and Adolescent Well-Being. Child Abuse \& Neglect, 33, 84-93.

Casanueva, C., Wilson, E., Smith, K., Dolan, M., Ringeisen, H., \& Horne, B. (2012). NSCAW II wave 2 report: child well-being (OPRE Report \#2012-38). Retrieved from the U.S. Department of Health and Human Services, Administration for Children and Families, Office of Planning, Research and Evaluation website: http://www.acf.hhs.gov/sites/default/files/opre/nscaw_report_w2_ch_wb_final_june_201 4_final_report.pdf 
Centers for Disease Control and Prevention (2008). Child maltreatment surveillance: Uniform definitions for public health and recommended data elements. Retrieved from http://www.cdc.gov/violenceprevention/pdf/cm_surveillance-a.pdf

Center for the Study of Social Policy. (2003). Raising educational achievement: Recommendations for state policy. Retrieved from http://www.cssp.org/publications/public-policy/policy-matters-raising-educationalachievement.pdf

Chaffin, M., Silovsky, J. F., Funderburk, B., Valle, L., Brestan, E. V., Balachova, T., Jackson, S., Lensgraf, J., \& Bonner, B. L. (2004). Parent-Child Interaction Therapy with physically abusive parents: Efficacy for reducing future abuse reports. Journal of Consulting and Clinical Psychology, 72, 500-510.

Chazan-Cohen, R., Raikes, H., Brooks-Gunn, J., Ayoub, C., Pan, B. A., Kisker, E. E., Roggman, L., \& Fuligni, A. S. (2009). Low-income children's school readiness: Parent contributions over the first five years. Early Education and Development, 20, 958-977.

Child Abuse Prevention and Treatment Reauthorization Act, P.L. 111-320, 42 U.S.C. $5101 \S 3$ (2010).

Cicchetti, D., \& Manly, T. (1990). A personal perspective on conducting research with maltreating families: Problems and solutions. In I. E. Sigel \& G. H. Brody (Eds.). Methods of family research: Biographies of research projects (pp. 87-134). Hillsdale, NJ: Lawrence Erlbaum.

Colder, C. R., Campbell, R. T., Ruel, E., Richardson, J. L., \& Flay, B. R. (2002). A finite mixture model of growth trajectories of adolescent alcohol use: Predictors and consequences. Journal of Consulting and Clinical Psychology, 70, 976-985. 
Cole, P. M., Dennis, T. A., Smith-Simon, K. E., \& Cohen, L. H. (2008). Preschoolers' emotion regulation strategy understanding: Relations with emotion socialization and child selfregulation. Social Development, 18, 324-352.

Cole, P. M., Armstrong, L. M., \& Pemberton, C. K. (2010). The role of language in the development of emotion regulation. In S. D. Calkins \& M. A. Bell (Eds), Child development at the intersection of emotion and cognition (pp. 59-77). Washington, DC: American Psychological Association.

Cole, P. M., Martin, S. E., \& Dennis, T. A. (2004). Emotion regulation as a scientific construct: Methodological challenges and directions for child development research. Child Development, 75, 317-333.

Combs-Ronto, L. A., Olson, S. L., Lunkenheimer, L. S., \& Sameroff, A. J. (2009). Interactions between maternal parenting and children's early disruptive behavior: Bidirectional associations across the transition from preschool to school entry. Journal of Abnormal Child Psychology, 37, 1151-1163.

Conger, D., \& Finkelstein, M. (2003). Foster care and school mobility. Journal of Negro Education, 72, 97-103.

Conger, D. \& Rebeck, A., (2001). How children's foster care experiences affect their education. Retrieved from Vera Institute of Justice website: http://www.vera.org/sites/default/files/resources/downloads/How_foster_care_affects_ed ucation.pdf.

Coohey, C., Renner, L. M., Hua, L., Zhang, Y. J., \& Whitney, S. D. (2011). Academic achievement despite child maltreatment: A longitudinal study. Child Abuse \& Neglect, 35, 688-699. 
Cook, K. F., Kallen, M. A., \& Amtmann, D. (2009). Having a fit: Impact of number of items and distribution of data on traditional criteria for assessing IRT's unidimensionality assumption. Quality of Life Research, 16, 447-460.

Counts, J. M., Buffington, E. S., Chang-Rios, K., Rasmussen, H. N., \& Preacher, K. J. (2010). The development and validation of the protective factors survey: A self-report measure of protective factors against child maltreatment. Child Abuse \& Neglect, 34, 762-772.

Cristofaro, T. N., \& Tamis-LaMonda, C. S. (2011). Mother-child conversations at 36 months and at pre-kindergarten: Relations to children's school readiness. Journal of Early Childhood Literacy, 12, 68-97.

Crozier, J. C., \& Barth, R. P. (2005). Cognitive and academic functioning in maltreated children. Children \& Schools, 27, 197-206.

Curran, P. J., Lee, T. H., Howard, A. L., Lane, S. T., \& MacCallum, R. C. (2012).

Disaggregating within-person and between-person effects in multilevel and structural equation growth models. In J. R. Harring \& G. R. Hancock (Eds.) Advances in longitudinal methods in education and the social and behavioral sciences (pp. 217-254). Charlotte, NC: Information Age Publishing.

Denham, S. A., Bassett, H. H., Thayer, S. K., Mincic, M., Sirotkin, Y.S, \& Zinsser, K. (2012). Observing preschoolers' social-emotional behavior: Structure, foundations, and prediction of early school success. Journal of Genetic Psychology, 173, 246-278. doi:

\section{$\underline{10.1080 / 00221325.2011 .597457}$}

Dollar, J. M., \& Stifter, C. A. (2012) Temperamental surgency and emotion regulation as predictors of childhood social competence. Journal of Experimental Child Psychology, 112, 178-194. 
Duncan, G., Claessens, A., Huston, A. C., Pagani, L. S., Engel, M., Sexton, H., Dowsett, C. J., Magnuson, K., Klebanov, P., Feinstein, L., Brooks-Gunn, J., \& Duckworth, K. (2007). School readiness and later achievement. Developmental Psychology, 43, 1428.1446.

Duncan, G. J., \& Magnuson, K. (2011). The nature and impact of early achievement skills, attention skills, and behavior problems. In G. J. Duncan \& R. J. Murnane (Eds.). Whither opportunity: Rising inequality, schools, and children's life chances (pp. 47-69). New York, NY: Russell Sage.

Ekas, N. V., Lickenbrock, D. M., \& Braungart-Rieker, J. M. (2013). Developmental trajectories of emotion regulation across infancy: Do age and the social partner influence temporal patterns. Infancy, 18, 729-754. doi:10.1111/infa.12003

Eigsti, I., \& Cicchetti, D. (2004). The impact of child maltreatment on expressive syntax at 60 months. Developmental Science, 7, 88-102.

Eisenberg, N., Fabes, R. A., Guthrie, I. K., \& Reiser, M. (2000). Dispositional emotionality and regulation: Their role in predicting quality of social functioning. Journal of Personality and Social Psychology, 78, 136-157.

Fantuzzo, J., Manz, P. H., \& McDermott, P. (1998). Preschool version of the social skills rating system: An empirical analysis of its use with low-income children. Journal of School Psychology, 36(2), 199-214. doi:10.1016/S0022-4405(98)00005-3

Farran, D. C. (2011). Rethinking school readiness. Exceptionality Education International, 21, 515.

Feely, M., Hayes, M. Mienko, J., Panlilio, C., \& Miyamoto, S. (2015, January). The diffusion of federal social policy mandates: The case of CAPTA, 1974. Paper presented at the 2015 Annual Conference of the Society for Social Work and Research, New Orleans, LA. 
Ferrer, E., Balluerka, N., \& Widaman, K. F. (2008). Factorial invariance and the specification of second-order latent growth models. Methodology, 4, 22-36.

Flay, B. R., Biglan, A., Boruch, R. F., Castro, F. G., Gottfredson, D., Kellam, S., Moscicki, E. K., Schinke, S., Valentine, J. C., \& Ji, P. (2005). Standards of evidence: Criteria for efficacy, effectiveness, and dissemination. Prevention Science, 6, 151-175.

Font, S., \& Maguire-Jack. K. (2013). Academic engagement and performance: Estimating the impact of out-of-home care for maltreated children. Children and Youth Services Review, $35,856-864$.

Gagné, P. E., \& Hancock, G. R. (2006). Measurement model quality, sample size, and solution propriety in confirmatory factor models. Multivariate Behavioral Research, 41, 65-83.

George, R. (2006). A cross-domain analysis of change in students' attitudes toward science and attitudes about the utility of science. International Journal of Science Education, 28, 571589. doi: 10.1080/09500690500338755

Graziano, P. A., Reavis, R. D., Keane, S. P., \& Calkins, S. D. (2007). The role of emotion regulation in children's early academic success. Journal of School Psychology, 45, 3-19.

Graziano, P. A., Reavis, R. D., Keane, S. P., \& Calkins, S. D. (2007). The role of emotion regulation in children's early academic success. Journal of School Psychology, 45, 3-19.

Greenwood, C. R., Bradfield, T., Kaminski, R., Linas, M., Carta, J. J., \& Nylander, D. (2011). The response to intervention (RTI) approach in early childhood. Focus on Exceptional Children, 43, 1-22.

Greenwood, C. R., Carta, J. J., Atwater, J., Goldstein, H., Kaminski, R., \& McConnell, S. (2012). Is a response to intervention (RTI) approach to preschool language and early literacy instruction needed? Topics in Early Childhood Special Education, 33, 48-64. 
Gresham, F.M. \& Elliot, S. N. (1990). SSRS: Social Skills Rating System. Circle Pines, MN: AGS

Gross, J. J., \& Thompson, R. A. (2007). Emotion regulation: Conceptual foundations. In J. J. Gross (Ed.), Handbook of emotion regulation (pp. 3-24). New York, NY: Guilford Press.

Halle, T. G., Hair, E. C., Wandner, L. D., \& Chien, N. C. (2012). Profiles of school readiness among four-year-old Head Start children. Early Childhood Research Quarterly, 27, 613626.

Hancock, G. R. (2006). Power analysis in covariance structure modeling. In G. R. Hancock \& R. O. Mueller (Eds.). Structural equation modeling: A second course (pp. 69-118). Greenwod, CT: Information Age Publishing, Inc.

Hancock, G. R., \& Buehl, M. M. (2008). Second-order latent growth models with shifting indicators. Journal of Modern Applied Statistical Methods, 7, 39-55.

Hancock, G. R., Harring, J. R., \& Lawrence, F. R. (2013). Using latent growth models to evaluate longitudinal change. In G. R. Hancock \& R. O. Mueller (Eds.), Structural equation modeling: A second course (2nd ed.) (pp. 309-341). Charlotte, NC: Information Age Publishing, Inc.

Hancock, G. R., Kuo, W., \& Lawrence, F. R. (2001). An illustration of second-order latent growth models. Structural Equation Modeling, 8, 470-489.

Harring, J. R. (2009). A nonlinear mixed effects model for latent variables. Journal of Educational and Behavioral Statistics, 34, 293-318.

Harring, J. R., Kohl, N., Silverman, R. D., \& Speece, D. L. (2012). A second-order conditionally linear mixed effects model with observed and latent variable covariates. Structural Equation Modeling, 19, 118-136. 
Haskett, M. E., Stelter, R., Proffit, K., \& Nice, R. (2012). Parent emotional expressiveness and children's self-regulation: Associations with abused children's school functioning. Child Abuse \& Neglect, 36, 296-307.

Healey, C. V., \& Fisher, P. A. (2011). Young children in foster care and the development of favorable outcomes. Children and Youth Services Review, 33, 1822-1830.

Herschell, A. D., Calzada, E. J., Eyberg, S. M., \& McNeil, C. B. (2002). Parent-child interaction therapy: New directions in research. Cognitive and Behavioral Practice, 9, 9-15.

Hipp, J.R., \& Bauer, D.J. (2006). Local solutions in the estimation of growth mixture models. Psychological Methods, 11, 36-53. doi:10.1037/1082-989X.11.1.36.

Hix-Small, H., Duncan, T. E., Duncan, S. C., \& Okut, H. (2004). A multivariate associative finite growth mixture modeling approach examining adolescent alcohol and marijuana use. Journal of Psychopathology \& Behavioral Assessment, 26, 255-270.

Holland, P. (1986). Statistics and causal inference. Journal of the American Statistical Association, 81, 945-960.

Hu, L., \& Bentler, P. M. (1999) Cutoff criteria for fit indexes in covariance structure analysis: Conventional criteria versus new alternatives. Structural Equation Modeling, 6, 1-55. Individuals With Disabilities Education Act, 20 U.S.C. $§ 1400$ (2004).

Institute of Medicine and National Research Council. (2014). New directions in child abuse and neglect research. Washington, DC: The National Academies Press.

Jaffee, S. R., \& Maikovich-Fong, A. K. (2011). Effects of chronic maltreatment and maltreatment timing on children's behavior and cognitive abilities. The Journal of Child Psychology and Psychiatry, 52, 184-194. 
Jones Harden, B. (2004). Safety and stability for foster children: A developmental perspective. The Future of Children, 14, 39-47.

Jones Harden, B., \& Klein, S. (2011). Infants/toddlers in child welfare: What have we learned and where do we go from here? Children and Youth Services, 33, 1464-1468.

Jones Harden, B., Morrison, C., \& Clyman, R. (2014). Emotion labeling among preschool children in foster care. Early Education and Development, 25, 1180-1197.

Jones Harden, B., \& Whittaker, J. V. (2011). The early home environment and developmental outcomes for young children in the child welfare system. Children and Youth Services Review, 33, 1392-1403.

Kagan, S.L., Moore, E., \& Bredekamp, S. (1995). Reconsidering children's early development and learning: Toward common views and vocabulary. Washington, DC: National Education Goals Panel.

Kalpidou, M. D., Power, T. G., Cherry, K. E., \& Gottfried, N. W. (2004). Regulation of emotion and behavior among 3- and 5-year-olds. The Journal of General Psychology, 2, 159-178.

Kaplan, D. (2009). Structural equation modeling: Foundations and extensions ( ${ }^{\text {nd }}$ ed.). Thousand Oaks, CA: SAGE Publications, Inc.

Kaufman, A. S., \& Kaufman, N. L. (1990). Manual for the Kaufman Brief Intelligence Test. Circle Pines, MN: American Guidance Service.

Keenan, K., \& Shaw, D. (1997). Developmental and social influences on young girls' early problem behavior. Psychological Bulletin, 121, 95-113.

Kendall-Tackett, K. A., \& Eckenrode, J. (1996). The effects of neglect on academic achievement and disciplinary problems: A developmental perspective. Child Abuse \& Neglect, 20, 161-169. 
Kerbow, D. (1996). Patterns of urban student mobility and local school reform. Journal of Education for Students Placed at Risk, 1, 147-169.

Kim, S., Nordling, J. K., Yoon, J. E., Boldt, L. J., \& Kochanska, G. (2013). Effortful control in "hot" and "cool" tasks differentially predicts children's behavior problems and academic performance. Journal of Abnormal Child Psychology, 41, 43-56.

Kim-Spoon, J., Cicchetti, D., \& Rogosch, F. A. (2013). A longitudinal study of emotion regulation, emotion lability-negativity, and internalizing symptomatology in maltreated and non-maltreated children. Child Development, 84, 512-527. doi:10.1111/j.14678624.2012.01857.x

Kline, R. B. (2011). Principles and practice of structural equation modeling ( $3^{\text {rd }}$ ed.). New York, NY: Guilford Press.

Koran, J., \& Hancock, G. R. (2010). Using fixed thresholds with grouped data in structural equation modeling. Structural Equation Modeling, 17, 590-604. doi:10.1080/10705511.2010.510047

Kotch, J. B., Browne, D. C., Ringwalt, C. L., Dufort, V., Ruina, E., Stewart, P. W., \& Jung, J. (1997). Stress, social support, and substantiated maltreatment in the second and third years of life. Child Abuse \& Neglect, 21, 1025-1037.

Lanier, P., Maguire-Jack, K., Mienko, J., \& Panlilio, C. (2015). From causes to outcomes: Determining prevention can work. In D. Daro, A. C. Donnelly, L. A. Huang, \& B. Powell (Eds.) Advances in child abuse prevention knowledge (pp. 121 - 144). New York, NY: Springer.

Lanza, S. T., Coffman, D. L., \& Xu, S. (2013). Causal inference in latent class analysis. Structural Equation Modeling, 20, 361-383. doi:10.1080/10705511.2013.797816 
Laursen, B., \& Hoff, E. (2006). Person-centered and variable-centered approaches to longitudinal data. Merrill-Palmer Quarterly, 52, 377-389.

Leerkes, E. M., Paradise, M. J., O’Brien, M., \& Calkins, S. D. (2008). Emotion and cognition processes in preschool children. Merrill-Palmer Quarterly, 54, 102-124.

Leiter, J. (2007). School performance trajectories after the advent of reported maltreatment. Children and Youth Services Review, 29, 363-382.

Leiter, J., \& Johnsen, J. (1997). Child maltreatment and school performance declines: An eventhistory analysis. American Educational Research Journal, 34, 563-589.

Lerner, R. M., Theokas, C., \& Bobek, D. L. (2005). Concepts and theories of human development: Historical and contemporary dimensions. In M. H. Bornstein \& M. E. Lamb (Eds.). Developmental science: An advanced textbook (5 ${ }^{\text {th }}$ Edition, pp. 3-43). Mahwah, NJ: Lawrence Erlbaum Assoc.

Little, R. J. A., \& Rubin, D. B. (1989). The analysis of social science data with missing values. Sociological Methods and Research, 18, 292-326.

Lloyd, E. C., \& Barth, R. P. (2011). Developmental outcomes after five years for foster children returned home remaining in care, or adopted. Children and Youth Services Review, 33, 1383-1391.

Long, J. D., Harring, J. R., Brekke, J. S., Test, M. A., \& Greenberg, J. (2007). Longitudinal construct validity of Brief Symptom Inventory subscales in Schizophrenia. Psychological Assessment, 19, 298-308. doi:10.1037/1040-3590.19.3.298

Luke, N., \& Banerjee, R. (2013). Differentiated associations between childhood maltreatment experiences and social understanding: A meta-analysis and systematic review. Developmental Review, 33, 1-28. 
MacLean, P. C., Rynes, K. N., Aragón, C., Caprihan, A., Phillips, J. P., \& Lowe, J. R. (2014). Mother-infant mutual eye gaze supports emotion regulation in infancy during the StillFace paradigm. Infant Behavior \& Development, 37, 512-522. doi:10.1016/j.infbeh.2014.06.008

Maier,M. F., Vitiello, V. E., \& Greenfield, D. B. (2012). A multilevel model of child- and classroom-level psychosocial factors that support language and literacy resilience of children in Head Start. Early Childhood Research Quarterly, 27, 104-114.

Manly, J. T. (2005). Advances in research definitions of child maltreatment. Child Abuse \& Neglect, 29, 425-439.

Manly, J. T., Kim, J. E., Rogosch, F. A., \& Cicchetti, D. (2001). Dimensions of child maltreatment and children's adjustment: Contributions of developmental timing and subtype. Development and Psychopathology, 13, 759-782.

Maughan, A., \& Cicchetti, D. (2002). Impact of child maltreatment and inter-adult violence on children's emotion regulation abilities and socioemotional adjustment. Child Development, 73, 1525-1542.

Martins, E. A. (2012). Can We Identify Emotion Over-regulation in Infancy? Associations with Avoidant Attachment, Dyadic Emotional Interaction and Temperament. Infant \& Child Development, 21, 579-595.

McArdle, J.J. (1988). Dynamic but structural equation modeling of repeated measures data. In J.R. Nesselroade \& R.B. Cattell (Eds.). The handbook of multivariate experimental psychology: Perspectives on individual differences ( $2^{\text {nd }}$ ed., pp. 561-614). New York, NY: Plenum Press. 
McWayne, C. M., \& Bulotsky-Shearer, R. J. (2013). Identifying family and classroom practices associated with stability and change of social-emotional readiness for a national sample of low-income children. Research in Human Development, 10, 116-140. doi:10.1080/15427609.2013.786537

McWayne, C. M., Cheung, K., Wright, L. E. G., \& Hahs-Vaughn, D. L. (2012). Patterns of school readiness among Head Start children: Meaningful within-group variability during the transition to kindergarten. Journal of Educational Psychology, 104, 862-878.

McWayne, C. M., Green, L. E., \& Fantuzzo, J. W. (2009). A variable- and person-oriented investigation of preschool competencies and Head Start children's transition to kindergarten and first grade. Applied Developmental Science, 13, 1-15.

McWayne, C. M., Hahs-Vaughn, D. L., Cheung, K., \& Wright, L. E. G. (2012). National profiles of school readiness skills for Head Start children: An investigation of stability and change. Early Childhood Research Quarterly, 27, 668-683.

Mehta, P. D., Neale, M. C., \& Flay, B. R. (2004). Squeezing interval change from ordinal panel data: latent growth curves with ordinal outcomes. Psychological Methods, 9, 301-333. doi:10.1037/1082-989X.9.3.301

Mersky, J. P., Topitzes, J. D., \& Reynolds, A. J. (2011). Maltreatment prevention through early childhood intervention: A confirmatory evaluation of the Chicago Child-Parent Center preschool program. Children and Youth Services Review, 33, 1454-1463.

Miller, A. L., Gouley, K. K., Seifer, R., Dickstein, S., \& Shields, A. (2010). Emotions and behaviors in the Head Start classrooms: Associations among observed dysregulation, social competence, and preschool adjustment. Early Education and Development, 15, $147-166$. 
Mistry, R. S., Benner, A. D., Biesanz, J. C., Clark, S. L., \& Howes, C. (2010). Family and social risk, and parental investments during the early childhood years as predictors of lowincome children's school readiness outcomes. Early Childhood Research Quarterly, 25, 432-449.

Moreno, A. J., \& Robinson, J. L. (2005). Emotional vitality in infancy as a predictor of cognitive and language abilities in toddlerhood. Infant and Child Development, 14, 383-402.

Muthén, B. O. (2004). Latent variable analysis: Growth mixture modeling and related techniques for longitudinal data. In D. Kaplan (Ed.). The sage handbook of quantitative methodology for the social sciences. Thousand Oaks, CA: Sage Publications, Inc.

Muthén, L. K. (2010, February 11). Re: Model fit index WRMR [Online forum comment]. Retrieved from http://www.statmodel.com/discussion/messages/9/5096.html?1321986275

Muthén, B. O., \& Muthén, L. K. (2000). Integrating person-centered and variable-centered analyses: Growth mixture modeling with latent trajectory classes. Alcoholism: Clinical and Experimental Research, 24, 882-891.

Muthén, L.K., \& Muthén, B.O. (1998-2012). Mplus User’s Guide. Seventh Edition. Los Angeles, CA: Muthén \& Muthén.

Muthén, L. K., \& Muthén, B. O. (2009). How to use a Monte Carlo study to decide on sample size and determine power. Structural Equation Modeling, 9, 599-620.

Nagin, D. S. (1999). Analyzing developmental trajectories: A semiparametric, group-based approach. Psychological Methods, 4, 139-157. 
Nagin, D. S., \& Odgers, C. L. (2012). Group-based trajectory modeling in Developmental Science. In B. Laursen \& T. D. Little (Eds.). Handbook of developmental research methods. New York, NY: Guilford Press.

National Research Council and Institute of Medicine (2000) From neurons to neighborhoods: The science of early childhood development. Committee on Integrating the Science of Early Childhood Development. Jack P. Shonkoff and Deborah A. Phillips (Eds.) Board on Children, Youth, and Families, Commission on Behavioral and Social Sciences and Education. Washington, D.C.: National Academy Press.

National Research Council (2001) Eager to learn: Educating our preschoolers. committee on early childhood pedagogy. Barbara T. Bowman, M. Suzanne Donovan, and M. Susan Bums (Eds.). Commission on Behavioral and Social Sciences and Education. Washington, DC: National Academy Press.

National Survey on Child and Adolescent Well-Being (NSCAW; 2008). Data File User's Manual.

National Survey on Child and Adolescent Well-Being (NSCAW; 2008). Statistical Analysis User's Manual.

No Child Left Behind Act of 2001, 20 U.S.C. $§ 6301$ et seq. (2003).

Nylund, K. L. (2007). Latent transition analysis: Modeling extensions and an application to peer victimization (Doctoral dissertation). Available from ProQuest Dissertations and Theses database

Nylund, K. L., Asparouhov, T., \& Muthén, B. O. (2007). Deciding on the number of classes in latent class analysis and growth mixture modeling: A Monte Carlo simulation study. Structural Equation Modeling, 14, 535-569. 
O'Mahen, H. A., Karl, A., Moberly, N., \& Fedock, G. (2015). The association between childhood maltreatment and emotion regulation: Two different mechanisms contributing to depression? Journal of Affective Disorders, 174, 287-295. doi:10.1016/j.jad.2014.11.028

Pan, B. A., Rowe, M. L., Spier, E., \& Tamis-LeMonda, C. (2004). Measuring productive vocabulary of toddlers in low-income families: Concurrent and predictive validity of three sources of data. Journal of Child Language, 31, 587-608.

Panlilio, C., Jones Harden, B., Harring, J., \& Morrison, C. I. (2015). Estimating fear regulation profiles among young children in foster care: An exploratory study using latent class growth models. Manuscript in preparation.

Pears, K. C., \& Fisher, P. A. (2005). Emotion understanding and theory of mind among maltreated children in foster care: Evidence of deficits. Development and Psychopathology, 17, 47-65.

Pears, K. C., Fisher, P. A., Bruce, J., Kim, H. K., \& Yoerger, K. (2010). Early elementary school adjustment of maltreated children in foster care: The roles of inhibitory control and caregiver involvement. Child Development, 81, 1550-1564.

Pears, K. C., Heywood, C. V., Kim, H. K., \& Fisher, P. A. (2011). Pre-reading deficits in children in foster care. School Psychology Review, 40, 140-148.

Pears, K. C., Kim, H. K., \& Fisher, P. A. (2008). Psychosocial and cognitive functioning of children with specific profiles of maltreatment. Child Abuse \& Neglect, 32, 958-971.

Perlman, S., \& Fantuzzo, J. (2010). Timing and influence of early experiences of child maltreatment and homelessness on children's educational well-being. Children and Youth Services Review, 32, 874-883. 
Petras, H., \& Masyn, K. (2010). General growth mixture analysis with antecedents and consequences of change. In A. R. Piquero \& D. Weisburd (Eds.). Handbook of quantitative criminology. New York, NY: Springer Science \& Business Media, LLC.

Pianta, R. C. (2007). Early education in transition. In R. C. Pianta, M. J. Cox, \& K. L. Snow (Eds.). School readiness \& the transition to kindergarten in the era of accountability (pp. 3-10). Baltimore, MD: Paul H. Brookes Publishing.

Posner, M. I., \& Rothbart, M. K. (2000). Developing mechanisms of self-regulation. Development and Psychopathology, 12, 427-441.

Ram, N., Grimm, K. J., Gatze-Kopp, L. M., \& Molenar, P. C. M. (2012). Longitudinal mixture models and the identification of archetypes. In B. Laursen \& T. D. Little (Eds.). Handbook of developmental research methods. New York, NY: Guilford Press.

Raver, C. C. (2002). Emotions matter: Making the case for the role of young children's emotional development for early school readiness. Retrieved from: http://harrisschool.uchicago.edu/about/publications/working-papers/pdf/wp_02_06.pdf.

Raver, C. C., Garner, P. W., \& Smith-Donald, R. (2007). The role of emotion regulation and emotion knowledge for children' academic readiness. In R. C. Pianta, M. J. Cox, \& K. L. Snow (Eds.). School readiness \& the transition to kindergarten in the era of accountability (pp. 121-148). Baltimore, MD: Paul H. Brookes Publishing.

Raver, C. C., Jones, S. M., Li-Grining, C. P., Metzger, M., Champion, K. M., \& Sardin, L. (2008). Improving preschool classroom processes: Preliminary findings from a randomized trial implemented in Head Start settings. Early Childhood Research Quarterly, 23, 10-26. 
Raver, C. C., Jones, S. M., Li-Grining, C. P., Zhai, F., Bub, K., \& Pressler, E. (2011). CSRP's impact on low-income preschoolers' preacademic skills: Self-regulation as a mediating mechanism. Child Development, 82, 362-378.

Research and Training Center on Community Living. (2013). A taxonomy for postsecondary education programs for students with intellectual and developmental disabilities (Policy Research Brief \# 23). Minneapolis, MN: University of Minnesota, Institute on Community Integration.

Research Triangle Institute. (2008). From early involvement with child welfare services to school entry: A 5- to 6-year follow-up of infants in the National Survey of Child and Adolescent Well-Being. Research Triangle Park, NC: RTI International.

Rindskopf, D. (2009). Latent class analysis. In R. E. Millsap, A. Maydeu-Olivares, R. E. Millsap, A. Maydeu-Olivares (Eds.), The Sage handbook of quantitative methods in psychology (pp. 199-215). Thousand Oaks, CA: Sage Publications Ltd.

Robinson, L., Morris, A., Heller, S., Scheeringa, M., Boris, N., \& Smyke, A. (2009). Relations between emotion regulation, parenting, and psychopathology in young maltreated children in out of home care. Journal of Child \& Family Studies, 18, 421-434.

Romens, S. E., \& Pollak, S. D. (2012). Emotion regulation predicts attention bias in maltreated children at-risk for depression. The Journal of Child Psychology and Psychiatry, 53, 120127.

Rouse, H. L., \& Fantuzzo, J. W. (2009). Multiple risks and educational well-being: A population-based investigation of threats to early school success. Early Childhood Research Quarterly, 24, 1-14. 
Rowe, E., \& Eckenrode, J. (1999). Timing of academic difficulties among maltreated and nonmaltreated children. Child Abuse \& Neglect, 23, 813-832.

Rueda, M. R., Posner, M. I., \& Rothbart, M. K. (2005). The development of executive attention: Contributions to the emergence of self-regulation. Developmental Neuropsychology, 28, 573-594.

Rumberger, R., \& Larson, K. A. (1998). Student mobility and the increased risk of high school dropout. American Journal of Education, 107, 1-35.

Rumberger, R. (2003). The causes and consequences of student mobility. Journal of Negro Education, 72, 6-21.

Ryan, J., \& Testa, M. (2005). Child maltreatment and juvenile delinquency: Investigating the role of placement and placement stability. Children and Youth Services Review, 22, 227249.

Samuelsen, K., \& Dayton, C. M. (2010). Latent class analysis. In G. Hancock \& R. Mueller (Eds.) Quantitative methods in the social and behavioral sciences: A guide for researchers and reviewers. Mahwah, NJ: Erlbaum

Scarborough, A. A., \& McCare, J. S. (2010). School-age special education outcomes of infants and toddlers investigated for maltreatment. Children and Youth Services Review, 32, 8088.

Schelble, J. L., Franks, B. A., \& Miller, M. D. (2010). Emotion dysregulation and academic resilience in maltreated children. Child Youth Care Forum, 39, 289-303.

Schrank, F. A., McGrew, K. S., \& Woodcock, R. W. (2001). Technical Abstract (WoodcockJohnson III Assessment Service Bulletin No. 2). Itasca, IL: Riverside Publishing. 
Sedlak, A.J., Mettenburg, J., Basena, M., Petta, I., McPherson, K., Greene, A., and Li, S. (2010). Fourth National Incidence Study of Child Abuse and Neglect (NIS-4): Report to Congress. Washington, DC: U.S. Department of Health and Human Services, Administration for Children and Families.

Sektnan, M., McClelland, M. M., Acock, A., \& Morrison, F. J. (2010). Relations between early family risk, children's behavioral regulation, and academic achievement. Early Childhood Research Quarterly, 25, 464-479.

Slade, E. P., \& Wissow, L. S. (2007). The influence of childhood maltreatment on adolescents' academic performance. Economics of Education Review, 26, 604-614.

Smithgall, C., Gladden, R. M., Howard, E., George, R., \& Courtney, M. (2004). Educational experiences of children in out-of-home care. Chicago: Chapin Hall Center for Children.

Son, S., \& Morrison, F. J. (2010). The nature and impact of changes in home learning environment on development of language and academic skills in preschool children. Developmental Psychology, 46, 1103-1118.

Song, L., Spier, E. T., \& Tamis-LeMonda, C. S. (2014). Reciprocal influences between maternal language and children's language and cognitive development in low-income families. Journal of Child Language, 41, 305-326.

Spencer, T. J., Faraone, S. V., Surman, C. B. H., Petty, C., Clarke, A., Batchelder, H., Wozniak, J., \& Biederman, J. (2011). Toward defining deficient emotional self-regulation in children with Attention-Deficit/Hyperactivity Disorder using the Child Behavior Checklist. Postgraduate Medicine, 123. doi: 10.3810/pgm.2011.09.2459 
Spritz, B. L., Sandberg, E. H., Maher, E., \& Zajdel, R. T. (2010). Models of emotion skills and social competence in the Head Start classroom. Early Education and Development, 21, 495-516.

Stacks, A. M., \& Partridge, T. (2011). Infants placed in foster care prior to their first birthday: Differences in kin and non-kin placement. Infant mental Health Journal, 32, 489-508.

Stahmer, A. C., Leslie, L. K., Hurlburt, M., Barth, R. P., Webb, M. B., Landsverk, J., \& Zhang, J. (2005). Developmental and behavioral needs and service use for young children in child welfare. Pediatrics, 116, 891-900.

Stapleton, L. M. (2002). The incorporation of sample weights into multilevel structural equation models. Structural Equation Modeling, 9, 475-502. doi: 10.1207/S15328007SEM0904_2

Stapleton (2006). Using multilevel structural equation modeling techniques with complex sample data. In G. R. Hancock \& R. O. Mueller (Eds.). Structural equation modeling: A second course (pp. 345-384). Greenwod, CT: Information Age Publishing, Inc.

Stock, C. D. \& Fisher, P. A. (2006). Language delays among foster children: Implications for policy and practice. Child Welfare, $85,445-461$.

Stone, S. (2007). Child maltreatment, out-of-home placement and academic vulnerability: A fifteen-year review of evidence and future directions. Children and Youth Services Review, 29, 139-161.

Thelen, E., \& Smith, L.B. (2006) Dynamic Systems Theories. In W. Damon \& R. M. Lerner (Eds.), Handbook of child psychology, Vol. 1: Theoretical models of human development (6 $6^{\text {th }}$ ed., pp. 258-312). New York: Wiley.

Thomas, R., \& Zimmer-Gembeck, M. J. (2012). Parent-Child Interaction Therapy: An evidencebased treatment for child maltreatment. Child Maltreatment, 17(3), 253-266. 
Thompson, R. A. (1994). Emotion regulation: A theme in search of definition. Monographs of the Society for Research in Child Development, 59, 25-52.

Timmer, S. G., Urquiza, A. J., Zebell, N. M., \& McGrath, J. M. (2005). Parent-Child Interaction Therapy: Application to maltreating parent-child dyads. Child Abuse \& Neglect, 29(7), $825-842$.

Tisak, J., \& Meredith, W. (1990). Descriptive and associative developmental models. In A. von Eye (Ed.), Statistical methods in developmental research, volume 2 (pp. 387-406). San Diego, CA: Academic Press.

Totsika, V., \& Sylva, K. (2004). The HOME observation for measurement of the environment revisited. Child and Adolescent Mental Health, 9, 25-35.

Tudge, J. R. H., Mokrova, I., Hatfield, B. E., \& Karnik, R. B. (2009). Uses and misuses of Bronfenbrenner's Bio-ecological theory of human development. Journal of Family Theory \& Review, 1, 198-210.

Ursache, A., Blair, C., Stifter, C., \& Voegtline, K. (2013). Emotional reactivity and regulation in infancy interact to predict executive functioning in early childhood. Developmental Psychology, 49, 127-137. doi:10.1037/a0027728

U.S. Department of Health and Human Services, Administration for Children and Families, Administration on Children, Youth and Families, Children's Bureau. (2011). Child Maltreatment 2010. Retrieved from http://www.acf.hhs.gov/programs/cb/stats_research/index.htm\#can.

U.S. Department of Health and Human Services, Administration for Children and Families, Administration on Children, Youth and Families, Children's Bureau. (2012). Child 
Maltreatment 2011. Retrieved from http://www.acf.hhs.gov/programs/cb/research-datatechnology/statistics-research/child-maltreatment.

Vacca, J. S. (2008). Foster children need more help after they reach the age of eighteen. Children and Youth Services Review, 30, 485-492.

Vitiello, V. E., Greenfield, D. B., Munis, P., \& George, J. (2011). Cognitive flexibility, approaches to learning, and academic school readiness in Head Start preschool children. Early Education and Development, 22, 388-410.

von Eye, A., \& Bergman, L. R. (2003). Research strategies in developmental psychopathology:

Dimensional identity and the person-oriented approach. Development and Psychopathology, 15, $553-580$.

von Eye, A., \& Bogat, G. A. (2006). Person orientation: Concepts, results, and development. Merrill Palmer Quarterly, 52, 390-420.

Wang, M., \& Bodner, T. E. (2007). Growth mixture modeling: Identifying and predicting unobserved subpopulations with longitudinal data. Organizational Research Methods, 10, 635-656. doi: 10.1177/1094428106289397

Webb, T. L., Miles, E., \& Sheeran, P. (2012). Dealing with feeling: A meta-analysis of the effectiveness of strategies derived from the process model of emotion regulation. Psychological Bulletin, 138, 775-808.

Wechsler-Zimring, A. T. (2012). Posttraumatic stress disorder and removal from home as a primary, secondary, or disclaimed trauma in maltreated adolescents. Journal of Family Violence, 27, 813-818.

Wendling, B. J., Schrank, F. A., \& Schmitt, A. J. (2007). Educational interventions related to the Woodcock-Johnson III tests of achievement (Assessment Service Bulletin No. 8). Rolling Meadows, IL: Riverside Publishing. 
Whitney, S. D., Renner, L. M., \& Herrenkohl, T. I. (2010). Gender differences in risk/protection profiles for low academic performance. Journal of Community Psychology, 38, 435-455.

Williford, A. P., Whittaker, J. E. V., Vitiello, V. E., \& Downer, J. T. Children's engagement within the preschool classroom and their development of self-regulation. Early Education and Development, 24, 162-187.

Woodcock, R. W., McGrew, K. S., \& Mather, N. (2001). Woodcock Johnson III tests of achievement. Rolling Meadows, IL: Riverside Publishing.

Woodruff, K., \& Lee, B. (2011). Identifying and predicting problem behavior trajectories among pre-school children investigated for child abuse and neglect. Child Abuse \& Neglect, 35, 491-503.

Wulczyn, F. (2009). Epidemiological perspectives on maltreatment prevention. The Future of Children, 19, 39-66.

Wulczyn, F., Kogan, J., \& Harden, B. (2003). Placement stability and movement trajectories. Social Service Review, 77, 212-236.

Wulczyn, F., Barth, R. P., Yuan, Y. T., Jones Harden, B., \& Landsverk, J. (2005). Beyond common sense: Child welfare, child well-being, and the evidence of policy reform. Piscataway, NJ: Aldine Transaction.

Yu, C. (2002). Evaluating cutoff criteria of model fit indices for latent variable models with binary and continuous outcomes (Doctoral dissertation). Available from ProQuest Dissertations and Theses database

Yu, E., Day, P., \& Williams, M. (2002). Improving educational outcomes for youth in care: A national collaboration. Washington, D.C.: Child Welfare League of America. 
Zhai, F., Raver, C. C., Jones, S. M., Li-Grining, C. P., Pressler, E., Gao, Q. (2010). Dosage effects on school readiness: Evidence from a randomized classroom-based intervention. Social Services Review, 615-654.

Zimmer, M. H., \& Panko, L. M. (2006). Developmental status and service use among children in the child welfare system: A national survey. Archives for Pediatric and Adolescent Medicine, 160, 183-188.

Zimmerman, L. K., \& Stansbury, K. (2003). The influence of temperamental reactivity and situational context on the emotion-regulatory abilities old 3-year-old children. The Journal of Genetic Psychology, 164, 389-409.

Zimmerman, I. L., Steiner, V. G., \& Pond, R. E. (1992). PLS-3: Preschool Language Scale-3. San Antonio, TX: The Psychological Corporation.

Zingraff, M. T., Leiter, J., \& Johnsen, M. C. (1994). The mediating effect of good school performance on the maltreatment-delinquency relationship. Journal of Research in Crime \& Delinquency, 31, 62-91.

Zorc, C. S., O'Reilly, A. R., Matone, M., Long, J., Watts, C. L., \& Rubin, D. (2013). The relationship of placement experience to school absenteeism and changing schools in young, school-aged children in foster care. Children \& Youth Services Review, 35, 826833. 